\title{
Physics of laser heated ferromagnets: Ultrafast demagnetization and magneto-Seebeck effect
}

\author{
Dissertation \\ zur Erlangung des mathematisch-naturwissenschaftlichen \\ Doktorgrades \\ "Doctor rerum naturalium" \\ der Georg-August-Universität Göttingen
}

\author{
vorgelegt von \\ Jakob Walowski \\ aus Ketrzyn (Polen)
}

Göttingen, 2012 
Referent: Prof. Dr. Markus Münzenberg Korreferent: Prof. Dr. Konrad Samwer

Tag der mündlichen Prüfung: 5. März 2012 


\section{Contents}

Introduction v v

$\begin{array}{lll}1 & \text { Ultrafast Spin Dynamics } & 1\end{array}$

1.1 The Basis of Magnetization Dynamics . . . . . . . . . . . . . . 2

1.1.1 Near Equilibrium Spin Precession . . . . . . . . . . . . . . . . 2

1.1 .2 Forces Acting in Ferromagnets . . . . . . . . . . . . . . . . . 3

1.1 .3 Microscopic Spin-Flip Processes . . . . . . . . . . . . . . . . . . 5

1.2 The Thermal Model . . . . . . . . . . . . . . . . . . . . . . 7

1.2.1 The Landau-Lifshitz-Bloch Equation LLB . . . . . . . . . . 7

$1.2 .2 \quad$ Magnetization Dynamics, Approaching $T_{C} \ldots \ldots . . . . .11$

1.3 The Experimental Procedure . . . . . . . . . . . . . . . . . . 15

$1.3 .1 \quad$ LLB Equation and Experimental Data . . . . . . . . . . . . . 15

1.3.2 The Two Temperature 2T and Three Temperature 3T Model. 17

1.3.3 Determining the Spin Temperature via the 2T Model . . . . . 18

1.4 Experimental Results . . . . . . . . . . . . . . . . . 23

1.4 .1 Penetration Depth of Light into Metals . . . . . . . . . . . . . 23

$1.4 .2 \quad$ Absorbed Laser Power Density . . . . . . . . . . . . . . . . . 23

$1.4 .3 \quad$ Reflectivity Data and Electron Temperatures . . . . . . . . . . . 27

$1.4 .4 \quad$ Demagnetization Data and Spin Temperatures . . . . . . . . . . 32

$1.4 .5 \quad$ Limitation of the 3T Fitting Procedure . . . . . . . . . . . . . 39

1.4 .6 More Approaches to Magnetization Dynamics Beyond $T_{C}$. . . 41

1.5 Concluding Remarks . . . . . . . . . . . . . . . . 47

1.5 .1 Summary . . . . . . . . . . . . . . . . . . . . . . . . . . . . . . . . . . . . . 47

1.5.2 $\quad$ Outlook . . . . . . . . . . . . . . . . . . . . . . . . . . 48

\begin{tabular}{|lll}
2 & Magneto-Seebeck Effect in Tunnel Junctions & 51
\end{tabular}

2.1 Thermoelectric Effects . . . . . . . . . . . . . . . 51

$2.2 \quad$ Irreversible Thermodynamics and Thermodynamic Transport. . . . . 52

2.2 .1 The Dynamical Equations and Kinetic Coefficients . . . . . . 55

2.2 .2 The Seebeck Effect . . . . . . . . . . . . . . . . . . 56

2.2 .3 The Peltier Effect $\ldots \ldots \ldots$. . . . . . . . . . . . . . . . . . . . . 58

2.2 .4 The Thomson Effect . . . . . . . . . . . . . . . . . . . . . . . 59

2.2 .5 Thermo-magneto-electric Dynamical Equations . . . . . . . . 60

2.3 Phenomenological Discussion $\ldots \ldots \ldots \ldots$. . . . . . . . . . . 66

2.3 .1 Thermal Conductivity and Thermoelectric Power . . . . . . . 66 


\section{Contents}

2.4 Recent Advances in Spincaloritronics . . . . . . . . . . . . . . . . . . 71

$2.4 .1 \quad$ Magneto-Seebeck Effect in GMR Multilayers . . . . . . . . . . 71

2.4 .2 Spin-Seebeck Effect . . . . . . . . . . . . . . . . . . . . 72

2.4 .3 Spin-Seebeck and Magneto-Seebeck Effect . . . . . . . . . . . 73

2.4 .4 Experimental Approaches to the Magneto-Seebeck Effect . . . 79

2.5 Measurement of the Magneto-Seebeck Effect in TMR Junctions . . . 81

2.5 .1 The Experimental Setup . . . . . . . . . . . . . . . . 81

2.5 .2 Signal Optimization Process . . . . . . . . . . . . . . . . . 83

2.5.3 The Examined Samples . . . . . . . . . . . . . . . . . 85

2.6 Experimental Results . . . . . . . . . . . . . . . . . . . 87

2.6 .1 The Temperature Gradient . . . . . . . . . . . . . . . . . . . 87

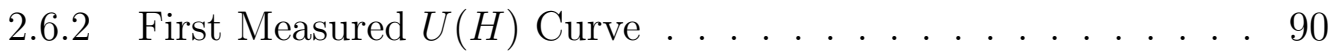

2.6.3 Temperature Dependent Seebeck Voltage in Large Sized Tunnel Junctions . . . . . . . . . . . . . . . . . . . . . 92

2.6 .4 Modulation Techniques . . . . . . . . . . . . . . . . . 96

2.6 .5 Determining the Sign of the Seebeck Voltage . . . . . . . . . . 98

2.6.6 Final Experimental Results and Simulations . . . . . . . . . . 100

2.7 Concluding Remarks . . . . . . . . . . . . . . . . 116

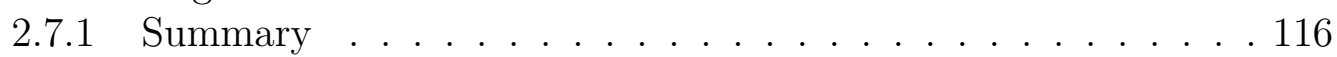

2.7 .2 Outlook . . . . . . . . . . . . . . . . . . . . 116

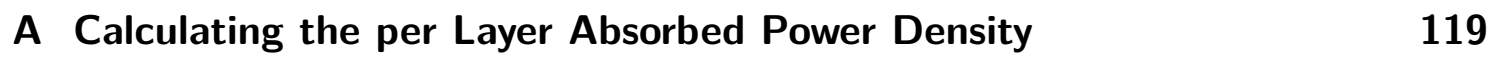

B TRMOKE and Time-Resolved $\Delta R$ Experimental Data 121

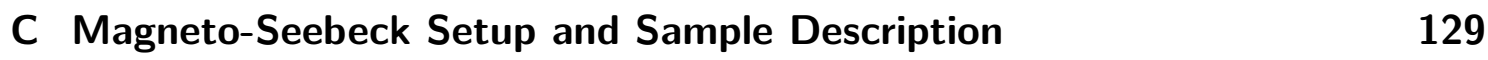




\section{Introduction}

The neologism 'spintronics' emerged in the end of the 1980s. After Johnson and Silsbee managed to inject a spin polarized current into a normal metal in 1985 [35] and the discovery of giant magneto resistance (GMR) by Albert Fert and Peter Grünberg [7, 11] in 1988, the potential to combine the two properties of an electron, namely the charge and the spin, was recognized. This opened a wide and very active research field looking for the basic mechanisms of magnetism and electronics and trying to implement these properties into logic and memory devices. The availability of ultrafast femtosecond pulsed Ti:Sapphire lasers pushed the field of magnetization dynamics to regions, at which spin-flips can be detected in the time domain, revealing the temporal limits of spintronics devices.

After the discovery of the GMR followed by the tunnel magneto resistance (TMR) and their commercial success in the hard drive industry, the potential to work on ever smaller devices shifted the focus of research into the field of spintronics. This extended interest led to a neglection of further visionary works by Johnson and Silsbee for the following $\sim 15$ years. They set up a theory for spin polarized electron transport (transport of magnetization), driven by the force generated by temperature gradients, coupling electron currents to heat currents [36]. With decreasing device sizes into nanometer regions and thus increasing current densities to critical magnitudes causing high temperatures during operation, Moore's Law threatens to break down in current electronic appliances. Around 5 years ago this bottleneck brought the ideas of Johnson and Silsbee back to the scene again, and extended the research field to heat-driven spintronics. The neologism was enhanced and has been dubbed 'spin caloritronics'. The implementation of thermo-electric effects combined with magnetism will provide the capability to reuse the heat produced by microchips and increase efficiency. One future vision is to build devices that contain microchip technology based on thermo-magneto-electric effects that are powered by the touch of ones hand. In its final state and at high efficiency levels, microchips based on these effects might be powered by generating a temperature gradient provided by body heat at one side, while keeping the other side cool. However, at this stage of development, this is more an illusion than a road map for future applications.

The present work is divided into two Chapters. In Chapter one, the first systematic study of magnetization dynamics triggered by ultrashort laser pulses is presented for polycrystalline nickel films with thicknesses ranging from $2 \mathrm{~nm}$ to $40 \mathrm{~nm}$, measured at fluences from $10 \mathrm{~mJ} / \mathrm{cm}^{2}$ to $50 \mathrm{~mJ} / \mathrm{cm}^{2}$. The thermal model, from which the Landau-Lifshitz-Bloch equation is derived, is shown as an deterministic approach to describe spin dynamics processes even above the Curie temperature in the time regime $(<1 \mathrm{ps}-100 \mathrm{ps})$. By averaging over electronic properties, spin excitation 
and relaxation processes in real ferromagnetic thin films can be predicted by using the electron temperature as the only time-dependent input parameter obtained from experiments. For the $15 \mathrm{~nm}$ nickel film, simulation results are compared to experimental data for increasing pump fluences. The challenges with the determination of the electron temperature arising from the experimental technique are discussed for the remaining nickel film thicknesses. An analytical equation based on the three temperature model is used to determine relaxation times from the dynamics experiments for all measured nickel films, which are then compared with the predictions drawn from the LLB equation. Finally, the experimental results are discussed in the focus of other models being currently developed for the description of ultrafast magnetization dynamics processes. In the end, an outlook is given, pointing out an extension of the experimental technique, and further experiments, which promise answers to still open questions.

Chapter two deals with the recently re-established research field under the new title 'spin caloritronics'. First, the description of thermovoltages based on irreversible thermodynamics is given, pointing out the magnetic contribution in magnetic tunnel junctions (MTJs), refurbished from Johnson's and Silsbee's works from the late 1980s. These voltages can be utilized to store and read binary data by only applying temperature gradients across the MTJs. Then, the examined magnetic tunnel junctions and their properties are described, followed by a detailed explanation of the experiment planning as well as construction, and the experimental procedure to measure the magneto-Seebeck effect in those junctions by generating a temperature gradient using an optical heat source (laser). Following that, the final experiments are compared to ab-initio calculations predicting the upper limits of the magnetoSeebeck effect to be above $1000 \%$ in $\mathrm{MgO}$-based magnetic tunnel junctions. In the end, an outlook on how the experimental technique can be improved, and how the tunnel junctions can be optimized to better suit this experiment is suggested. The implementation of these suggestions should improve the experimental data and enable more precise conclusions, which can be drawn quicker and give a better understanding of these effects in the future.

The most challenging task in the analysis of the magneto-Seebeck effect in MTJs is the access to the temperature and temperature gradient across the tunneling layer situated below several other metallic layers. Both, the temperature and the temperature gradient are determined by modeling a MTJ and simulating the heat distribution after the deposition of the laser energy using heat capacities and heat conductivity as input parameters. The expertise in the determination of temperature from reflectivity measurements discussed in the first part is a promising technique, which may be applied to obtain experimental data of the temperature dynamics on the surface of MTJs. These data will provide more realistic input parameters about the heat distribution for simulations and thus lead to more precise data of the temperature and the temperature gradient inside of the MTJ. Besides that, a better understanding of the mechanisms underlying the demagnetization and relaxation processes will provide the temporal limits at which magnetization reversal, required to write information, is possible. 


\section{Ultrafast Spin Dynamics}

All-optical pump-probe [10] and optical pump x-ray magnetic circular dichroism probe [57] experiments are the ultimate methods to gain insight into magnetization dynamics on ultrashort time scales. Recently, there has been substantial research interest on short time scale dynamics $(\sim 30 \mathrm{ps})$, applying both experimental techniques [38, 6, 65, 52, to gain more insight into the processes driving the ultrafast demagnetization and relaxation, after heating ferromagnets with ultrashort laser pulses. These processes are taking place at time scales near the resolution limit of these experimental techniques, and their interpretation and theoretical modeling is of tremendous importance for the understanding of the underlying micromagnetic mechanisms.

Even though the processes and the undergoing mechanisms on time scales considered long $(\sim 1 \mathrm{~ns})$ in these experiments are better understood, they are still an important tool in the search for new materials for future spintronics applications [46, 62].

These experimental methods do not allow the observation of single electrons or their spins. In order to unite the experimental observations, which are usually averaging over $10^{12}-10^{20}$ electron spins, and the theoretically calculated behavior of single electron spins, approximations are necessary. There are currently three theoretical approaches dealing with the explanation of these ultrafast processes. In 2009, Koopmans et al. [38] developed the microscopic three temperature model (m3TM) based on exchange interaction, and averaging electronic properties, to describe the ultrafast demagnetization in transition metals, and slower demagnetization in $5 \mathrm{f}$ rare earths, like gadolinium. Half a year later, in 2010, Battiato et al. [8] suggested a superdiffusive model, based on electronic effects, averaging magnetic degrees of freedom.

Below, the analysis focuses on magnetization dynamics of thin nickel films $5 \mathrm{~nm}-$ $40 \mathrm{~nm}$ on the short time scales. Nickel is chosen for the analysis, because it is a commonly used representative material exhibiting ultrafast demagnetization, on which the other models under discussion have been tested and compared to experimental data. Therefore it offers the largest potential for comparison to other current work on this topic. The theoretical description is given by the Landau-Lifshitz-Bloch equation (thermal model), since this seems to be the most promising model, describing these processes. Following that, the experimental results are discussed in the light of the two other suggested models. 


\subsection{The Basis of Magnetization Dynamics}

\subsection{The Basis of Magnetization Dynamics}

\subsubsection{Near Equilibrium Spin Precession}

Relaxation processes on long time scales, beginning at $t \approx 30$ ps are near equilibrium small perturbation processes. They take place, after the excitation processes have extinguished and can be neglected. One sufficient possibility to describe these dynamic processes is the Landau-Lifshitz-Gilbert equation (LLG) of precessional motion:

$$
\frac{d \vec{M}}{d t}=-\gamma\left[\vec{M} \times \vec{H}_{\mathrm{eff}}\right]+\frac{\alpha}{M_{S}}\left[\vec{M} \times \frac{d \vec{M}}{d t}\right],
$$

with $\vec{M}$, the magnetization vector of constant length, $\gamma=-g \mu_{B} \mu_{0} / \hbar$ determining the precession frequency, $\vec{H}_{\text {eff }}$ the effective field, consisting of the field contributions described below. The dimensionless parameter $\alpha$, is the phenomenological damping constant, describing the decay of the precession amplitude. The first part of the right hand side can be derived from quantum mechanics for a single spin $s$ from the time evolution of the Schrödinger equation in the Heisenberg picture, and also from classical mechanics, by describing a dipole moment in a current loop. Both derivations yield in similar forms, describing a torque, i.e. a spin in case of quantum mechanics or a magnetic moment in the classical description, rotating under the force of the external field. The connection of both, the classical and the quantum mechanical magnetic moments is done by expressing the quantum mechanical variables via their accessible expectation values and adding up the orbital and the spin momenta to one total magnetic moment $\vec{m}$. Summing up the magnetic moments over the unit volume results in the macro-spin $\vec{M}=\frac{\sum_{V} \vec{m}}{V}$, observed in experiments. In the equation of motion given above, the damping is included in the Gilbert form, hence the name LLG. In the original equation introduced by Landau and Lifshitz, the expression was:

$$
\frac{d \vec{M}}{d t}=-\gamma\left[\vec{M} \times \vec{H}_{\mathrm{eff}}\right]+\frac{\alpha}{M_{S}} \vec{M} \times\left[\vec{M} \times \vec{H}_{\mathrm{eff}}\right]
$$

an ordinary differential equation. Both forms can be transformed into each other. The latter is used as a starting point for the derivation of the Landau-Lifshitz-Bloch equation.

One solution to the LLG equation is simply given by $\vec{M}(t) \sim \exp \left(-t / \tau_{\alpha}\right) \sin (\omega t)$. It describes a ferromagnet under the boundary conditions of a thin film, leading to a vanishing in-plane anisotropy, the external field slightly turning the samples magnetization $\vec{M}$ out of the film plane. Only one precession mode (Kittel mode) is occurring. From the relaxation process into the equilibrium position on long time scales $(\sim 1 \mathrm{~ns})$, the relaxation time $\tau_{\alpha} \sim 1 / \alpha$ and the precession frequency $\omega$ can be extracted, and allow the determination of the out of plane anisotropy [21]. In thicker, or structured films, where the shape anisotropies change, other additional precession modes, perpendicular standing spin-waves (PSSW), and Damon Eshbach 
modes can be observed. The interpretation of these modes is another research topic, of magnonics [41]. It deals with with spin wave-propagation, and the engineering of materials to be able to direct these waves into defined directions, in analogy to light waves in photonics.

\subsubsection{Forces Acting in Ferromagnets}

There are several forces acting on magnetic moments in ferromagnetic materials. The resulting acting field is the effective field $\vec{H}_{\text {eff }}$; it is the sum of all the magnetic fields, each arising from their magnetic force.

\section{Exchange Interaction}

The most fundamental underlying magnetic force stems from the exchange interaction which is ascribed to the Pauli-Principle. This states that no Fermions are allowed to match in all their quantum numbers. The resulting energy difference between two spins $\boldsymbol{s}_{i}$ and $\boldsymbol{s}_{j}$ is expressed by the Heisenberg-Hamiltonian:

$$
\mathcal{H}_{\text {heis }}=-\sum_{i \neq j}^{N} J_{i j} \boldsymbol{s}_{i} \cdot \boldsymbol{s}_{j}=-2 \sum_{i<j}^{N} J_{i j} \boldsymbol{s}_{i} \cdot \boldsymbol{s}_{j} .
$$

The exchange integral $J_{i j}=J_{j i}$ is symmetric, therefore it can be simplified as was done on the right hand side of the equation. If $J_{i j}>0$, ferromagnetic coupling minimizes the energy. This energy is very short ranged because of the overlap of the wave functions only over the distance of two atoms, therefore the summation over the nearest neighbors only is justified. Connecting $J_{i j}$ to the Weiss field by considering the magnetic moment, allows the spin alignment description of a ferromagnet to be described by its temperature dependence. Where the Curie-temperature $T_{C}$ determines the strength of spin coupling. The resulting exchange energy $E_{e x}$ is calculated by replacing the sum in equation 1.3 by an integral over the examined volume of the sample:

$$
E_{e x}=A \int_{V}(\nabla \vec{m})^{2} d V
$$

The material specific exchange constant $A=\frac{2 J s^{2}}{a}$ with $a$, the lattice constant, determines the strength of the coupling. $\vec{m}=\frac{\vec{M}}{M_{S}}$ is the magnetization normalized to the saturation magnetization. This integral describes the energy that keeps the spins aligned in one direction in ferromagnetic materials. That means, in the case of a weak perturbation of one spin, other neighboring spins will follow the motion. This is the energy that is responsible for the long range ordering and allows us to describe the experiments on long time scales by a macro spin approximation and the Landau-Lifshitz equation, because the spin can be considered rigidly coupled within the examined volume.

In fact, choosing ferromagnetic films with thicknesses in the vicinity of the pumppulse penetration depth, leads to spin excitations across the whole thickness of the 


\subsection{The Basis of Magnetization Dynamics}

film. These show in one uniform precession mode for the whole examined area on the long time scale.

\section{Magneto-Crystalline and Shape Anisotropy}

Additionally the experiments show a decay of the precession amplitude. This is the result of other energies acting in a ferromagnet. The exchange interaction forces spins to align parallel to each other or antiparallel, in the case of anti ferromagnets. However, it does not assign a specific direction. The magneto-crystalline anisotropy (arising from the crystal structure) and the shape anisotropy (arising from the shape of the sample) on the other hand force the spins, i.e. the magnetization $\vec{M}_{S}$ into a specific direction. The anisotropy energy defines an easy axis, the axis, along which $\vec{M}_{S}$ points, when no external force is applied, and a hard axis, which requires energy e.g. in form of an applied magnetic field, in order to turn $\vec{M}_{S}$ in its direction. The energy density $e_{a n i}$ can be expressed as a series of even powers, by neglecting terms of higher than the first order [59]:

$$
e_{a n i}=K_{1} \sin ^{2} \theta+\ldots
$$

where $K_{1}\left[\mathrm{~J} / \mathrm{cm}^{3}\right]$ is the anisotropy constant and $\theta$ is the enclosed angle between the saturation magnetization $\vec{M}_{S}$ and the unique axis. For thin films, the unique axis turns out to be the surface normal. The anisotropy field resulting from this energy density is

$$
H_{a n i}=\frac{2 K_{1}}{M_{S}} \cos \theta
$$

For a magnetization along the sample surface, $\theta=90^{\circ}$. This yields in one possibility, when $K_{1}>0$, the easy axis is out-of-plane, along the surface normal, and a second, when $K_{1}<0$, the easy axis is in-plane, along the sample surface. The anisotropy constant itself has two contributions again, $K_{1}=K_{u}+K_{s}$. The first one, $K_{u}$ is the contribution stemming from the magneto-crystalline part, which is the coupling of the isotropic spin moment and the anisotropic lattice. This anisotropy is usually difficult to estimate, because it can only be extracted from band structure calculations and is strongly temperature dependent. The second, $K_{s}$ is the contribution from the shape anisotropy. It is defined as:

$$
K_{s}=-\frac{1}{2 \mu_{0}} M_{S}^{2}
$$

This part of the anisotropy is limited to the saturation magnetization $M_{S}$. Therefore, whether a material has an in-plane or out-of-plane easy axis can be tailored by changing the amplitude of the magneto-crystalline anisotropy. Usually multilayer materials show a high magneto-crystalline anisotropy and hence an out-of-plane easy axis [59]. 


\section{Zeeman Energy}

The last contribution to be mentioned is the Zeeman energy $E_{Z}$, which is the interaction of the externally applied magnetic field $\vec{H}_{e x t}$ and the magnetization, given by:

$$
E_{Z}=-\mu_{0} \int_{V} \vec{M} \cdot \vec{H}_{e x t} d V
$$

The control over the external field and the knowledge of the easy axis, allows the alignment of the magnetization $\vec{M}$ to a specified direction. Using pump-probe experiments it is possible to perturb equilibrium and track the realignment process. A more detailed description of the long time scale processes, the derivation of the Landau-Lifshitz-Gilbert equation, and an analytic solution for thin films with inplane anisotropy is given in [21, 63]. The description of the magnetic energies contributing to build up a ferromagnetic system is taken from [59].

\subsubsection{Microscopic Spin-Flip Processes}

Experimentally, a macroscopic change of magnetization is observed. This macroscopic disorder from thermal excitations in the spin system has its origin in microscopic spin-flip processes, which take place on the femtosecond time scale.

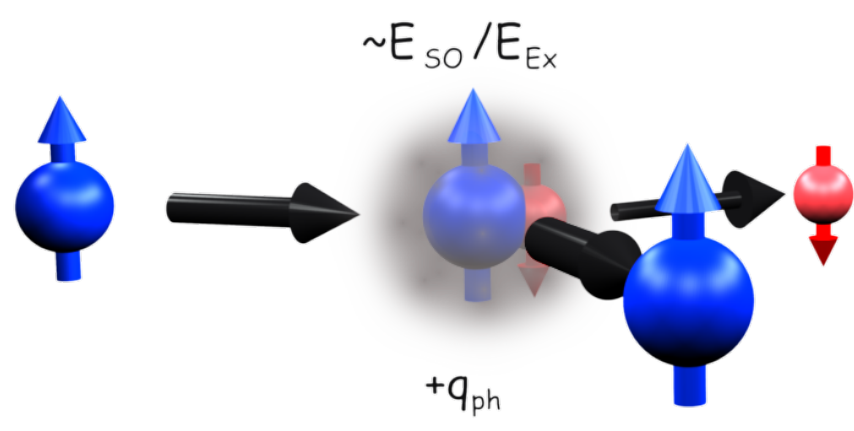

(a) A scattering event leading to an Elliott-Yafet spin-flip process.

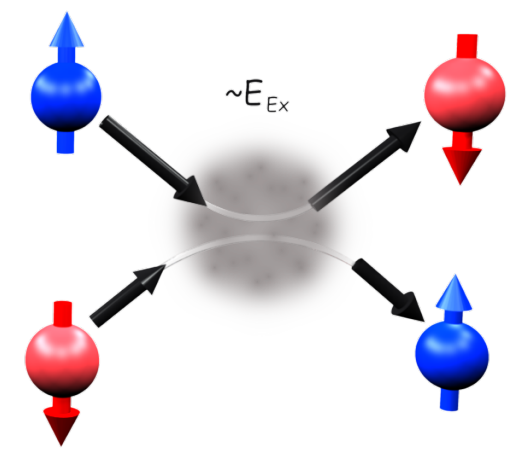

(b) An exchange scattering event leading to a spin-flip process.

Figure 1.1: Schematics of the two most predominant electron-scattering events, responsible for spin-flip processes.

In the current discussion, there are two relevant scattering events, responsible for spin-flip processes (see schematics in figure 1.1). First, the scattering of electrons at phonons (electron-phonon scattering). Second, the scattering of electrons at other electrons (electron-electron scattering).

The first event is sketched in figure 1.1(a), proceeding from left to right. An electron of a denoted spin state enters a scattering event. During the scattering event, there are two possible spin states, it occurs that the spin is in a mixed 


\subsection{The Basis of Magnetization Dynamics}

state. This leads to a possibility for the electron to leave the scattering event in the reversed state. This process for a spin-flip is called the Elliott-Yafet scattering event, proposed by Elliott in 1954 [24], and Yafet in 1963 [66]. This mechanism states that because of spin-orbit interaction, the electron spin ceases to be a good quantum number. Therefore, during the scattering event, the electron spin is in a mixed state of spin-up and spin-down. $|\Psi\rangle=a\left|\Psi_{\uparrow}\right\rangle+b\left|\Psi_{\downarrow}\right\rangle$. Assuming a denoted spin-up electron, $b$ is small for weak, and larger for strong spin-orbit coupling. That means, if highly excited electrons incur into scattering events with phonons or defects in the lattice structure, where the band structure exhibits high-symmetry points, the spin state is not conserved anymore, leading to a non-vanishing probability $b$ of a spinflip process. The energy and angular momentum is inherited by the phonon. The suitability of this mechanism, causing spin-flip processes of highly excited electrons after optical excitations with short femtosecond laser pulses in nickel, and that these processes take place at a femtosecond time scale has been calculated in [58. This process is the one that is responsible for the net magnetization decrease, after the excitation. It reduces the total magnetization of the excited material.

The second process, illustrated in figure 1.1(b), describes the event, when two electrons scatter at each other. A hot electron scatters at an electron of reversed spin at the Fermi level. After the scattering event, both electrons have reversed their spin, this spin-flip process ascribed to the exchange scattering. The formerly hot electron passes its energy to the electron at Fermi level, and relaxes to free lower energy states, while the electron formerly at the Fermi level, picks up the energy and stays in the energy region above the Fermi level. The net magnetization change is zero, but the now excited electron at around the Fermi level has a reversed spin. This electron will not be stable in its environment, and will further decay into lower energy spin excitation states. This subsequent relaxation follows a Stoner-type excitation process [6, 32]. 


\subsection{The Thermal Model}

\subsubsection{The Landau-Lifshitz-Bloch Equation LLB}

The description of the processes taking place on the short time scales can not be fulfilled by the mechanical macro-spin description of magnetization, where the magnetic moment is treated as a vector of fixed length and a temperature independent phenomenological damping parameter $\alpha$. Here the perturbation if chosen intense enough, i.e. if the pulses of the pump beam carry enough energy, can excite the electron system locally to temperatures even above the Curie point $T_{C}$. In that case, also the magnetization, or the spin system can be found in a highly non-equilibrium state.

One approach to model these processes is the thermal nondeterministic description of magnetization dynamics on the femtosecond time scale. Garanin [26] derived the Landau-Lifshitz-Bloch equation (LLB) starting with the description of a single classical spin $\boldsymbol{s}$ of a unit length under the impact of a fluctuating external field $\boldsymbol{H}$ by the stochastic Landau-Lifshitz equation:

$$
\dot{\boldsymbol{s}}=\gamma[\boldsymbol{s} \times(\boldsymbol{H}+\boldsymbol{\zeta})]-\gamma \lambda[\boldsymbol{s} \times[\boldsymbol{s} \times \boldsymbol{H}]] .
$$

Here $\gamma$ is the gyromagnetic ratio, $\lambda \ll 1$ expresses a weak coupling to the bath. The fluctuations are described by the Langevin field $\boldsymbol{\zeta}(t)$, which is given by its components:

$$
\left\langle\zeta_{\alpha}(t) \zeta_{\beta}\left(t^{\prime}\right)\right\rangle=\frac{2 \lambda T}{\gamma \mu_{0}} \delta_{\alpha \beta} \delta\left(t-t^{\prime}\right),
$$

where $\alpha, \beta=x, y, z$ represent the spatial directions, and $T$ is the temperature of the heat bath. From equation 1.5 it can be seen that the amplitude of the fluctuations increases with the temperature $T$ and the coupling strength $\lambda$ to the bath. The temporal distribution of the Langevin field is given by the probability distribution of random Gaussian noise. For equation 1.4 with the fluctuation of the Langevin field 1.5 a corresponding Fokker-Planck equation can be derived, using the dynamical renormalization-group theory. This is necessary, because magnetization is an average over a distribution function, whose magnitude can change. The exchange interaction between the external field $\boldsymbol{H}$ and the spin $\boldsymbol{s}$ is considered in the Fokker-Planck equation via the Hamiltonian $\mathcal{H}(\boldsymbol{s})=-\mu_{0} \boldsymbol{H} \boldsymbol{s}$, included in the distribution function. Using this Fokker-Planck equation, the equation of motion for the first moment $\boldsymbol{m} \equiv\langle\boldsymbol{s}\rangle$ of an assembly of magnetic atoms can be derived:

$$
\dot{\boldsymbol{m}}=\gamma[\boldsymbol{m} \times \boldsymbol{H}]-\Lambda_{N} \boldsymbol{m}-\gamma \lambda\langle[\boldsymbol{s} \times[\boldsymbol{s} \times \boldsymbol{H}]]\rangle .
$$

The characteristic diffusional relaxation rate is given by $\Lambda_{N} \equiv \tau_{N}^{-1} \equiv 2 \gamma \lambda T / \mu_{0}$. The last term of equation 1.6 couples the first moment to the second moments of the distribution function, therefore a temperature dependent reduced field needs to be introduced, which determines its behavior by: $\xi_{0} \equiv\left|\boldsymbol{\xi}_{\mathbf{0}}\right|$ and $\boldsymbol{\xi}_{\mathbf{0}} \equiv \mu_{0} \boldsymbol{H} / T$. Equation 1.6 can be evaluated for two cases. In the first case, when $\xi_{0} \gg 1$, i.e. in 


\subsection{The Thermal Model}

the low temperature limit, the second term can be neglected and for distribution functions localized about a given direction, $\left\langle s_{i} s_{j}\right\rangle \simeq m_{i} m_{j}$ decouples. Then the Landau-Lifshitz equation 1.4 for $\boldsymbol{m}$ is recovered without the stochastic fluctuations given by $\boldsymbol{\zeta}$. The second case, at high temperatures, when $\xi_{0} \ll 1$, the second term in equation 1.6 dominates and the equation of motion for $\boldsymbol{m}$ has a Bloch-like relaxation term $\sim \boldsymbol{m} / T$.

The next step is to investigate the behavior of the magnetization between those two limits, in the region where $\xi_{0} \sim 1$. In [25] it has been shown that for this intermediate region the Fokker-Planck equation can not be solved exactly, but an approximation, choosing the right distribution function leads to a solution with an accuracy of around $7 \%$ for $\xi_{0}=3$. There Garanin first gave exact solutions to the density matrix equation for a magnetic ion interacting with a phonon bath and later derived the Landau-Lifshitz-Bloch equation in which the relaxation parameters are expressed microscopically. The longitudinal relaxation rates in the exact solution have the Lorentzian form and an inverse integral relaxation time. The longitudinal relaxation rates of the Landau-Lifshitz-Bloch equation with the Lorentzian form have a deviation of at the most $7 \%$ from the exact relaxation rates. Nevertheless, in the high and low temperature limits, the Landau-Lifshitz-Bloch equation delivers exact solutions. In order to solve the Fokker-Planck equation for this intermediate region $(\xi \sim 1)$ a distribution function including the reduced field $\boldsymbol{\xi}(t)$ is needed. The solution is an equation of motion for the intermediate field with a longitudinal $\Gamma_{1}=\Lambda_{N} \frac{B(\xi)}{\xi B^{\prime}(\xi)}$ and a transverse $\Gamma_{2}=\Lambda_{N}\left(\frac{\xi}{B(\xi)}-1\right)$ relaxation rate:

$$
\dot{\boldsymbol{\xi}}=\gamma[\boldsymbol{\xi} \times \boldsymbol{H}]-\Gamma_{1}\left(1-\frac{\boldsymbol{\xi} \boldsymbol{\xi}_{\mathbf{0}}}{\xi^{2}}\right) \boldsymbol{\xi}-\Gamma_{2} \frac{\left[\boldsymbol{\xi} \times\left[\boldsymbol{\xi} \times \boldsymbol{\xi}_{\mathbf{0}}\right]\right]}{\xi^{2}} .
$$

Here the components of the relaxation rates are given by the Langevin function: $B(\xi)=\operatorname{coth} \xi-1 / \xi$ and $B^{\prime}(\xi) \equiv d B(\xi) / d \xi$. The equilibrium solution is given for $\boldsymbol{\xi}=\boldsymbol{\xi}_{0}$. Using the relation $\boldsymbol{m}=m \boldsymbol{\xi} / \xi$ and $m=B(\xi)$, the nonequilibrium solution is given by:

$$
\dot{\boldsymbol{m}}=\gamma[\boldsymbol{m} \times \boldsymbol{H}]-\Lambda_{N}\left(1-\frac{\boldsymbol{m} \boldsymbol{\xi}_{0}}{m \xi}\right) \boldsymbol{m}-\gamma \lambda\left(1-\frac{m}{\xi}\right) \frac{[\boldsymbol{m} \times[\boldsymbol{m} \times \boldsymbol{H}]]}{m^{2}} .
$$

The reduced field $\xi=\xi(m)$ is determined implicitly, by $m=B(\xi)$. Here again, as already shown in equation 1.6 , in the low temperature limit, $\xi \gg 1$, the transverse relaxation coefficient simplifies to $\gamma \lambda, \Lambda_{N}$ becomes small and looses impact. In the high temperature limit, when $\xi \ll 1$, the longitudinal relaxation coefficient gains importance, and the equation acquires the Bloch form. This equation, valid for a classical spin, was generalized for quantum spins in reference [25]. Both LLB equations, 1.7 and 1.8 can be put into a more convenient form, for small deviations out of the equilibrium, where $\boldsymbol{\xi}=\boldsymbol{\xi}_{0}$ and consequently $\boldsymbol{m} \cong \boldsymbol{m}_{0} \equiv B\left(\xi_{0}\right) \boldsymbol{\xi}_{0} / \xi_{0}$ :

$$
\dot{\boldsymbol{m}}=\gamma[\boldsymbol{m} \times \boldsymbol{H}]-\Gamma_{1}\left(1-\frac{\boldsymbol{m}_{\boldsymbol{m}}}{m^{2}}\right) \boldsymbol{m}-\Gamma_{2} \frac{\left[\boldsymbol{m} \times\left[\boldsymbol{m} \times \boldsymbol{m}_{0}\right]\right]}{m^{2}} .
$$


The longitudinal and transverse relaxation rates $\Gamma_{1}$ and $\Gamma_{2}$ are fluctuations of the reduced field $\xi_{0}$.

In a further step, the Fokker-Planck equation can be solved for fields $\boldsymbol{H}$ slowly changing in magnitude and direction. The result of the solution, the "slow" LandauLifshitz-Bloch equation differs from the instantaneous solution, only by the longitudinal relaxation terms. Both have the same asymptotes for high and low temperatures, but have a deviation of less than $7 \%$ in the remaining temperature range.

The description of real magnetic materials requires the inclusion of spin interaction between each other in the bath. Additionally, an anisotropy needs to be considered. This is done, by including a biaxially anisotropic exchange interaction into the Hamiltonian:

$$
\mathcal{H}=-\mu_{0} \sum_{i} \boldsymbol{H}_{i} \boldsymbol{s}_{i}-\frac{1}{2} \sum_{i j} J_{i j}\left(\eta_{x} s_{x i} s_{x j}+\eta_{y} s_{y i} s_{y j}+s_{z i} s_{z j}\right),
$$

with the anisotropy coefficients $\eta_{x} \leq \eta_{y} \leq 1$. The dynamics is described in the same way, as outlined above. Starting with the stochastic Landau-Lifshitz equation for a single spin $\boldsymbol{s}_{i}$, formulating a distribution function for the whole system of spins, and a Fokker-Planck equation for this distribution. The solution of the FokkerPlanck equation is rather sophisticated and requires many-body perturbation theory and mean-field approximation with spin-spin interactions included in the relaxation rates. The field $\boldsymbol{H}$ needs to be replaced by the molecular field given by $\boldsymbol{H}_{i}^{\mathrm{MFA}}=$ $-\frac{1}{\mu_{0}} \frac{\partial \mathcal{H}}{\partial \boldsymbol{s}_{i}}$. The single spins are replaced by the spin polarization $\boldsymbol{s}_{i} \Rightarrow \boldsymbol{m}_{i} \equiv\left\langle\boldsymbol{s}_{i}\right\rangle$. With the assumption of a small anisotropy $1-\eta_{x, y} \ll 1$, which is given in a ferromagnet, there is a small magnetization inhomogeneity at the distance of lattice spacing. In that case the continuous approximation is applicable and $\boldsymbol{H}_{i}^{\mathrm{MFA}}$ can be written as the sum of the effective filed $\boldsymbol{H}_{\text {eff }}^{\prime}$ and the exchange field $\boldsymbol{H}_{E}$ :

$$
\begin{array}{r}
\boldsymbol{H}^{\mathrm{MFA}}(\boldsymbol{r}) \cong \boldsymbol{H}_{E}+\boldsymbol{H}_{\mathrm{eff}}^{\prime}, \quad \boldsymbol{H}_{E}=\frac{J_{0}}{\mu_{0}} \boldsymbol{m}, \\
\boldsymbol{H}_{\mathrm{eff}}^{\prime}=\boldsymbol{H}+\frac{J_{0}}{\mu_{0}}\left[\alpha \Delta \boldsymbol{m}-\left(1-\eta_{x}\right) \boldsymbol{m}_{x}-\left(1-\eta_{x}\right) \boldsymbol{m}_{y}\right] .
\end{array}
$$

The parameters included are $J_{0}$, the zero order Fourier component of the exchange energy, $\Delta$ the Laplace operator, and $\alpha$ the lattice dependent constant, not the damping parameter. In ferromagnets, the exchange field is much stronger than the effective field $\left(\left|\boldsymbol{H}_{E}\right| \gg\left|\boldsymbol{H}_{\text {eff }}^{\prime}\right|\right)$. For temperatures below the Curie temperature it is the case, when $3 T_{C}=J_{0}$. For temperatures above $T_{C}$ there is a region, where the longitudinal susceptibility is large and also fulfills this condition. Then the external field $\boldsymbol{H}$ is not capable of changing the magnetization by large quantities and equation 1.9 can be used, where $\boldsymbol{m}_{0}=B_{j}\left(\beta \mu_{0} H^{\mathrm{MFA}}\right) \boldsymbol{H}^{\mathrm{MFA}} / H^{\mathrm{MFA}}$, with $\beta=1 / T$ is expanded up to the first order in $\boldsymbol{H}_{\text {eff }}^{\prime}$. For the average spin polarization per lattice site $\boldsymbol{m}(1 / N) \sum_{i=0}^{N}\left\langle\boldsymbol{s}_{i}\right\rangle$ this leads to the equation:

$$
\dot{\boldsymbol{m}}=\gamma\left[\boldsymbol{m} \times \boldsymbol{H}_{\mathrm{eff}}^{\prime}\right]-\gamma \lambda_{1}\left(\frac{1-B_{j} / m}{\mu_{0} \beta B_{j}^{\prime}}-\frac{\boldsymbol{m} \boldsymbol{H}_{\mathrm{eff}}^{\prime}}{m^{2}}\right) \boldsymbol{m}-\gamma \lambda_{2} \frac{\left[\boldsymbol{m} \times\left[\boldsymbol{m} \times \boldsymbol{H}_{\mathrm{eff}}^{\prime}\right]\right]}{m^{2}},
$$




\subsection{The Thermal Model}

with $B_{j}=B_{j=\infty}\left(m \beta J_{0}\right)$, the Langevin function, and $\lambda_{1}=2 \lambda \frac{T}{J_{0}}$ and $\lambda_{2}=\lambda\left(1-\frac{T}{J_{0}}\right)$ represent the longitudinal and transverse relaxation parameters $\Gamma_{1}$ and $\Gamma_{2}$ in equation 1.9. The first part in the second term of equation 1.10 represents the longitudinal relaxation. It can be approximated for different temperature regions by:

$$
\frac{1-B_{j} / m}{\mu_{0} \beta B_{j}^{\prime}} \cong \begin{cases}\frac{1}{2 \tilde{\chi}\|\|_{\|}}\left(\frac{m^{2}}{m_{e}^{2}}-1\right), & T<T_{C} \\ \frac{J_{0}}{\mu_{0}}\left(\frac{3}{5} m^{2}-\epsilon\right), & |\epsilon| \ll 1 .\end{cases}
$$

The second, lower case states the behavior around the Curie temperature, because $\epsilon \equiv 1-T / T_{C}$. The parameter $m_{e}$ gives the equilibrium spin polarization with $m_{e}=B_{j}\left(m_{e} \beta J_{0}\right)$, which will be the saturation magnetization at a given $T$ in the macroscopic case. The longitudinal susceptibility is given by:

$$
\tilde{\chi}_{\|}=\frac{\partial m}{\partial H}=\frac{\mu_{0}}{J_{0}} \frac{B_{j}^{\prime} \beta J_{0}}{1-B_{j}^{\prime} \beta J_{0}} .
$$

Both cases overlap in the region $\lesssim T_{C}$, delivering a description of motion for the whole temperature range. Finally the equation of motion for a macroscopic magnetization $\vec{M}=\mu_{0} \boldsymbol{m} / v_{0}$, the distribution of magnetic moments per unit cell volume $v_{0}$, is given by:

$$
\frac{d \vec{M}}{d t}=\gamma\left[\vec{M} \times \vec{H}_{\mathrm{eff}}\right]+L_{1} \frac{\left(\vec{M} \vec{H}_{\mathrm{eff}}\right) \vec{M}}{M^{2}}-L_{2} \frac{\left[\vec{M} \times\left[\vec{M} \times \vec{H}_{\mathrm{eff}}\right]\right]}{M^{2}} .
$$

$L_{1}, L_{2}$ are the longitudinal and the transverse relaxation coefficients respectively, determined by:

$$
L_{1,2}=\gamma M_{e} \alpha_{1,2}, \quad \alpha_{1,2}=\lambda_{1,2} / m_{e},
$$

where $\lambda$ is the coupling strength of the spins to the bath, and it also describes the coupling of the longitudinal and the transverse relaxation coefficients to each other. In equation 1.13, the longitudinal relaxation term 1.11 is already included in the effective field $\vec{H}_{\text {eff }}$. This derivation shows that starting with the description of a single randomly fluctuating spin, one can obtain a macroscopic description for temperature dependent spin dynamics in ferromagnets. In [6] this description was used to simulate the microscopic behavior of the magnetization to data obtained in alloptical pump-probe experiments with macroscopic access. Figure 1.2 outlines the relation between the microscopic and the macroscopic situation. In figure 1.2 (a), the microscopic situation is depicted. As mentioned above, in all-optical pump-probe experiments, the system can be excited out of equilibrium even above $T_{C}$, stirring up the spin system into a highly non-equilibrium state. This situation has been considered by including fluctuating Langevin fields into the Landau-Lifshitz equation as a starting point for the derivation of the LLB equation by Garanin [26]. The result for a ferromagnet with exchange interaction, and shape and crystalline anisotropy, is equation 1.13, describing the macrospin behavior. This equation includes two relaxation parameters with their two associated relaxation times. The first relaxation 


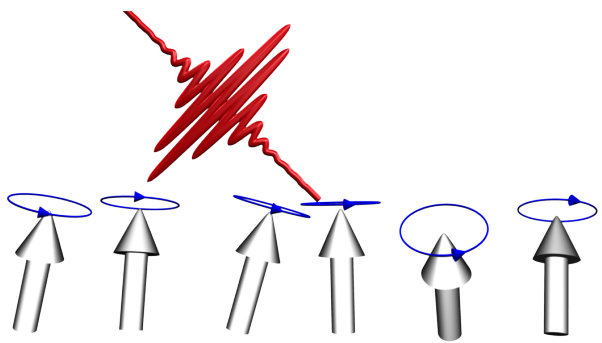

(a) The energy deposited by a laser pulse perturbs the spin system. The spins, coupled by exchange interaction to each other, and by anisotropy interaction to the heat bath are randomly fluctuating. This fluctuation is modeled by Langevin fields in the derivation of the Landau-Lifshitz-Bloch equation, describing the macroscopically accessible process.

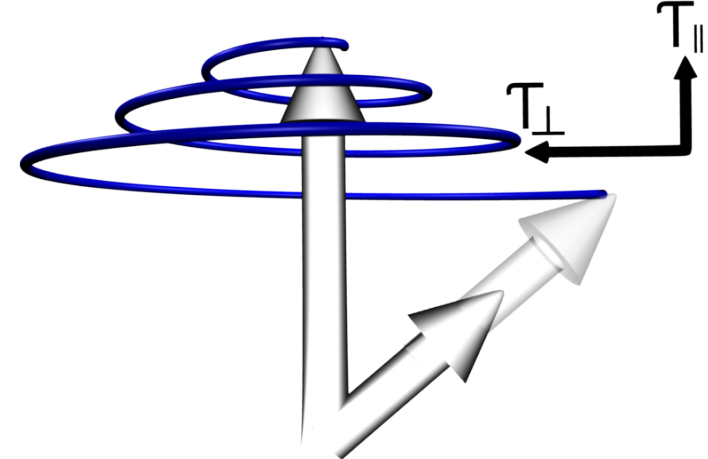

(b) The experimental access is only possible by averaging over $\gtrsim 10^{15}$ spins. In the thermal model, the description of random fluctuations results in a description by the LLB equation. It includes two relaxation times, one $\tau_{\|}$, acting parallel to the current direction of magnetization, and changing its magnitude. The other, $\tau_{\perp}$ acts perpendicular to the current magnetization vector direction.

Figure 1.2: The thermal model outlined schematically, showing the microscopic process (a) and the resulting macrospin picture (b). Adapted from [3].

parameter $L_{1}$ describes the relaxation pointing parallel to the current direction of the magnetization vector. It only plays a role around $T_{C}$, and is responsible for the change of the magnetization amplitude. The corresponding relaxation time is $\tau_{\|}$. The second relaxation parameter $L_{2}$ describes the relaxation pointing perpendicular to the current direction of the magnetization vector and perpendicular to the magnetizations current vector of motion. The corresponding relaxation time is $\tau_{\perp}$. The second relaxation term has the same form as the relaxations phenomenologically introduced by Landau and Lifshitz in equation 1.2 or the equivalent form introduced by Gilbert in equation 1.1. However, the nondeterministic relaxation parameter in the thermal model Landau-Lifshitz-Bloch equation 1.13 is temperature dependent and therefore valid for a wider temperature range. In the following section the behavior of the relaxation parameters at temperatures from below to above $T_{C}$ will be outlined.

\subsubsection{Magnetization Dynamics, Approaching $T_{C}$}

The deficiency of the Landau-Lifshitz-Gilbert equation in the regions close to $T_{C}$ has been investigated in [16]. It is shown there, how the LLB is advantageous in describing magnetization dynamics around $T_{C}$. The approach is similar to that of Garanin in [25, 26]. The starting point is an atomistic simulation based on the Landau-Lifshitz equation, in the same manner as in equation 1.4. The Langevin fields are included in the total field, as well as the exchange interaction and Zeeman 


\subsection{The Thermal Model}

contributions. The inclusion of the Zeeman field ensures a non-vanishing magnetization even above $T_{C}$.

Two test cases were simulated. In the first case, all spins pinned by an external field were simultaneously rotated by $30^{\circ}$ out of their equilibrium direction, for a temperature range from below up to above $T_{C}$. In that case the behavior of transverse relaxation $\tau_{\perp}$ was investigated. The result is an exponentially decaying sine oscillation with the transverse decay time $\tau_{\perp}$. By fitting the decaying oscillation function, the result is that for increasing temperatures below $T_{C}$ the relaxation also increases, i.e. the relaxation time $\tau_{\perp}$ decreases. Above $T_{C}$ on the other hand, the relaxation begins to decrease with increasing temperature, which means that the relaxation time $\tau_{\perp}$ increases. In the second case, the spins were rotated by an angle of $135^{\circ}$ out of equilibrium, which ensured that the projection of the external field onto the magnetization changed sign to negative, and was opposed to the exchange field. This opposite direction of both fields is responsible for the decrease of the absolute magnetization value $m=|\boldsymbol{m}|$ in the course of relaxation. In the data the decrease of $m$ shows up in a dip in the course of relaxation. The magnitude of the dip is $\Delta m \cong m(H, T)-m(0, T)$, where $H$ is the absolute value of the external magnetic field. That means that the absolute value of $m$ decreases by the difference of the equilibrium magnetization at $H=0$ and the magnetization at the actual given field. The second feature studied within this simulation set was the longitudinal relaxation time $\tau_{\|}$, which increases slowly with $T$. Around $T \leq T_{C}, \tau_{\|}$increases faster, until for temperatures $T \geq T_{C}$ it starts to decrease again. This quick increase of the longitudinal relaxation time $\tau_{\|}$in the vicinity of $T_{C}$ was recognized as the critical slowing down and ascribed to a second order transition.

In a next step, the Landau-Lifshitz-Bloch equation was solved, in order to test, whether it exhibits the same physical features, as the atomistic model simulations. The parameters applied in the calculation, the Curie temperature $T_{C}$, and the zerofield equilibrium magnetization $m_{e}$ were taken from the mean-field-approximation (MFA). Where $m=B_{\infty}\left(\beta\left(m J_{0}-\mu_{0} H\right)\right)$ is taken from the Curie-Weiss law with $B_{\infty}$ being the Langevin function in the approximation $B_{\infty}(x)=1 / 3 x-1 / 45 x^{3} \ldots$, and $\beta=1 / T$. The other parameters are as described in section 1.2.1. For magnetization dynamics calculations of real magnetic materials, these parameters can be taken as input data obtained from experiments. The results are shown in figure 1.3 . For the calculations, the LLB in the form (see equation 1.13)

$$
\dot{\boldsymbol{m}}=-\gamma[\boldsymbol{m} \times \boldsymbol{H}]+\gamma \alpha_{\|} \frac{\left[\boldsymbol{m} \cdot \boldsymbol{H}_{\mathrm{eff}}\right] \boldsymbol{m}}{m^{2}}-\gamma \alpha_{\perp} \frac{\left[\boldsymbol{m} \times\left[\boldsymbol{m} \times \boldsymbol{H}_{\mathrm{eff}}\right]\right]}{m^{2}}
$$

was applied. Both dimensionless longitudinal $\alpha_{\|}$and transverse $\alpha_{\perp}$ damping parameters are similar to the relaxation coefficients in equation 1.13 for $T<T_{C}$ :

$$
\alpha_{\|}=\lambda \frac{2 T}{3 T_{C}}, \quad \alpha_{\perp}=\lambda\left(1-\frac{T}{3 T_{C}}\right) .
$$

For $T>T_{C}$ the transverse damping parameter $\alpha_{\perp} \rightarrow \alpha_{\|}$. The coupling parameter $\lambda$ which connects both, the longitudinal and the transverse damping to the bath 


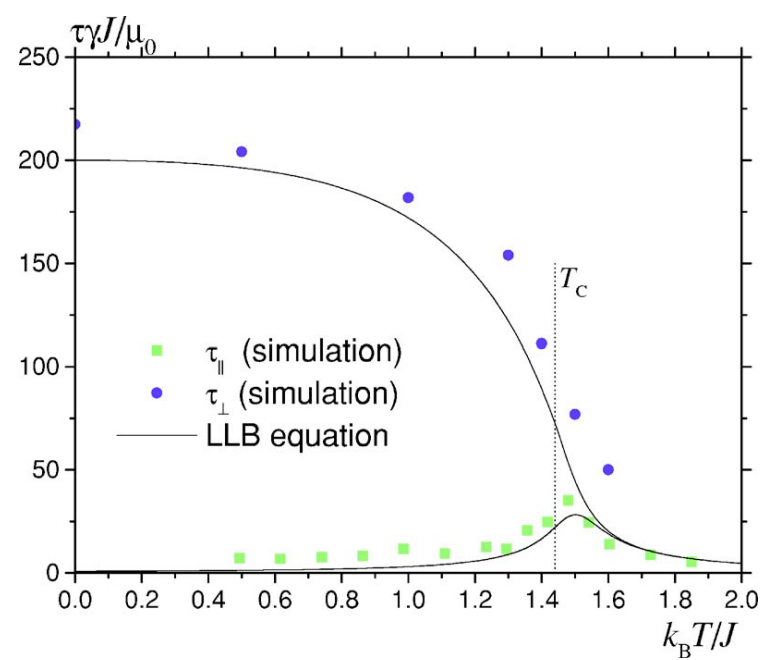

Figure 1.3: The longitudinal $\tau_{\|}$and transverse $\tau_{\perp}$ relaxation times versus the temperature. Calculated from the atomistic model simulations and by solving the Landau-Lifshitz-Bloch equation for different Temperatures far below, up to above $T_{C}$. Taken from [16].

has the same magnitude, as was used in the atomistic model simulation. In fact, $\lambda$ itself is also temperature dependent, but it is difficult to include this dependence into a semi-phenomenological approach, and besides that, leaving $\lambda$ temperature independent, makes the comparison with the atomistic model possible. The much weaker than the exchange interaction effective field $\boldsymbol{H}_{\text {eff }}$ contains the magnetization $\boldsymbol{m}$, which differs for the temperature range, as shown in the derivation outlined in section 1.2.1. the anisotropy field $\boldsymbol{H}_{A}=-\left(m_{x} \boldsymbol{e}_{x}+m_{y} \boldsymbol{e}_{y}\right) / \tilde{\chi}_{\perp}$, and the applied external field $\boldsymbol{H}$ :

$$
\boldsymbol{H}_{\mathrm{eff}}=\boldsymbol{H}+\boldsymbol{H}_{A}+ \begin{cases}\frac{1}{2 \tilde{\chi}_{\|}}\left(1-\frac{m^{2}}{m_{e}^{2}}\right) \boldsymbol{m}, & T \lesssim T_{C} \\ \frac{J_{0}}{\mu_{0}}\left(\epsilon-\frac{3}{5} m^{2}\right) \boldsymbol{m}, & T \gtrsim T_{C} .\end{cases}
$$

The second case, for temperatures around and above $T_{C}$ in the third term of $\boldsymbol{H}_{\text {eff }}$ can be rewritten, for the longitudinal susceptibility, in $-\frac{1}{\tilde{\chi}_{\|}}\left(1-\frac{3 T_{C}}{5\left(T-T_{C}\right)}\right) \boldsymbol{m}$.

A closer look for comparison of the LLB to the atomistic model shows the equivalence of both models, and hence the validity for the LLB even for elevated temperatures. Firstly, from equation 1.15 one can see that the longitudinal damping parameter monotonically increases with temperature, taking $\lambda$ as a constant, even linearly, while the transverse damping parameter decreases for temperatures below $T_{C}$ monotonically and increases again for temperatures above $T_{C}$. However, the change is very small over the temperature range up to $T_{C}$. Therefore the relaxation rates need to be evaluated, in order to compare the relaxation times of the LLB to the ones obtained form the atomistic simulations. Those are defined as follows:

$$
\Gamma_{\|}=\frac{\gamma \alpha_{\|}}{\tilde{\chi}_{\|}(H, T)}, \quad \Gamma_{\perp}=\frac{\gamma \alpha_{\perp}}{\tilde{\chi}_{\perp}(H, T)} .
$$




\subsection{The Thermal Model}

In order to gain an insight into the change of the relaxation rates and the inversely proportional relaxation times, a closer look at the susceptibilities and their behavior over the temperature range is necessary. From the definition of $\tilde{\chi}_{\|}$in equation 1.12 it can be seen that $\Gamma_{\|} \sim J_{0}$. Since the exchange interaction is very strong in a ferromagnet, the relaxation rate is high and therefore the relaxation time $\tau_{\|}$ is very low, as is also the case within the atomistic model. Around $T_{C}$ however, the longitudinal susceptibility increases, which decreases the relaxation rate $\Gamma_{\|}$, and increases $\tau_{\|}$. This critical slowing down is rather a feature of the temperature dependence of $\chi_{\|}$, than the variation of $\alpha_{\|}$. In the isotropic model, the transverse susceptibility is defined by $\tilde{\chi}_{\perp}=m(H, T) / H$. Therefore, the transverse relaxation rate $\Gamma_{\perp} \sim H$, is much weaker than the longitudinal relaxation rate. Additionally, $\Gamma_{\perp} \sim 1 / m(H, T)$, and since $m$ decreases with increasing temperature, also the relaxation rate increases. For the relaxation times $\tau_{\|, \perp}$ that means, at low temperatures, $\tau_{\|} \ll \tau_{\perp}$, but $\tau_{\|}$increases, and $\tau_{\perp}$ decreases with increasing temperature. At high temperatures around $T_{C}$ both relaxation rates approach each other very closely and merge for temperatures above $T_{C}$, as is illustrated in figure 1.3 .

The vanishing of $\chi_{\|}$at low temperatures is also the reason for the convergence of $m$ towards $m_{e}$, the equilibrium magnetization. Then the first relaxation term in equation 1.13 and equation 1.14 vanishes, and both equations merge into the Landau-Lifshitz-Gilbert equation. 


\subsection{The Experimental Procedure}

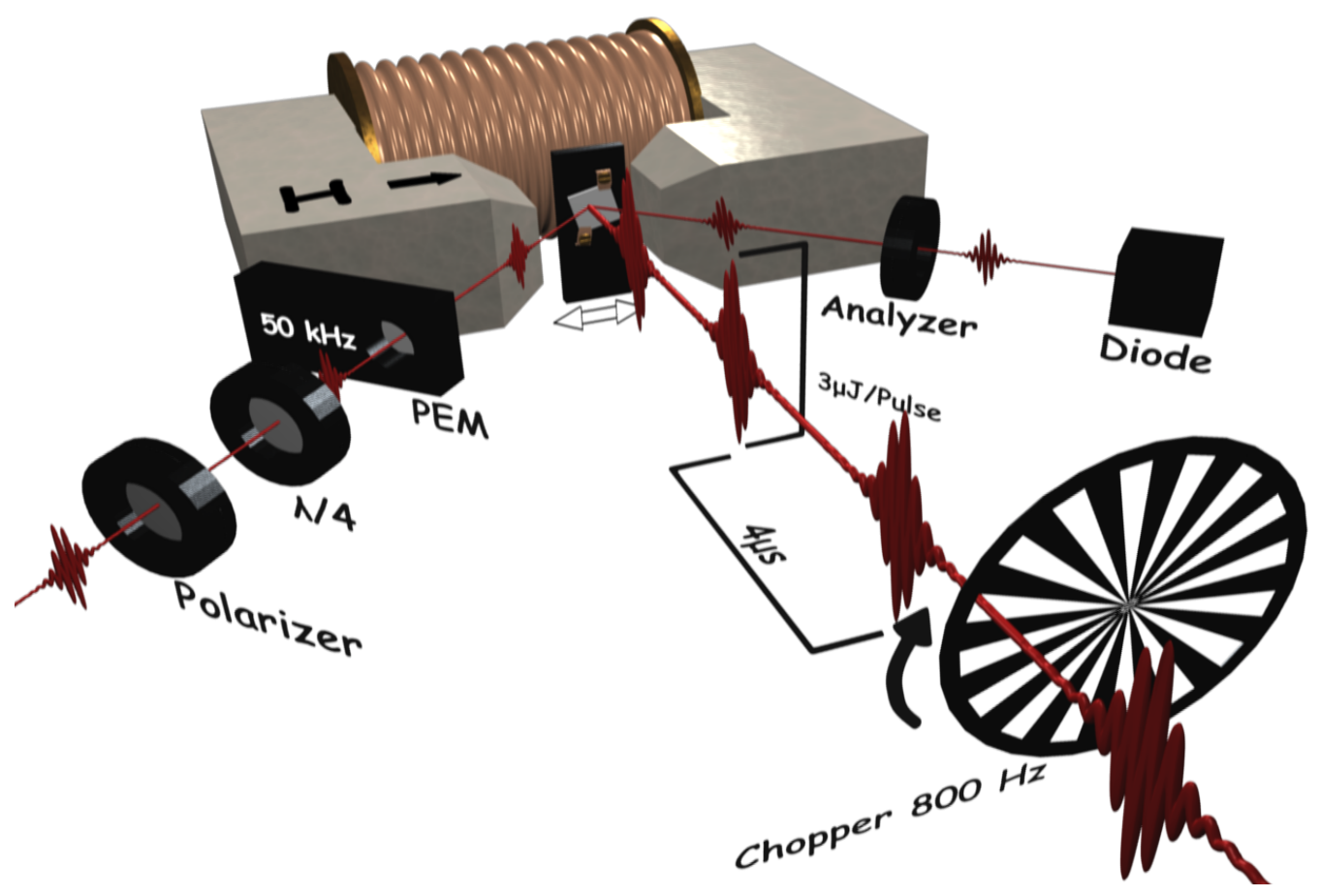

Figure 1.4: Schematics of the experimental setup to measure magnetization dynamics (TRMOKE) and time-resolved reflectivity $\Delta R(\Delta \tau)$. For details see text.

\subsubsection{LLB Equation and Experimental Data}

The verification of the Landau-Lifshitz-Bloch equation's ability to describe magnetization dynamics in real ferromagnets, requires a comparison with experimental data. The here discussed dynamic processes take place on a sub-picosecond time scale, and therefore demand excitations and probe durations that are at least one order of magnitude shorter. So far only femtosecond pulsed Ti:Sapphire lasers reach laser pulse lengths below $100 \mathrm{fs}$. The state of the art until now are pump-probe experiments, whether all-optical or probed using synchrotron radiation. These techniques provide the access to processes on the femtosecond time scale and should mirror the dynamics described by the LLB closely. They show, the change of magnetization at a given time, compared to the saturated equilibrium state at the samples initial temperature (usually room temperature), after the ferromagnet absorbed the energy of an ultrashort laser pulse. A time resolution of $\sim 40 \mathrm{fs}$ nowadays is standard, but shorter pulse lengths, even below $5 \mathrm{fs}$ are available. Moreover, these experiments are capable of raising the spin temperature above $T_{C}$ almost instantly, i.e. within $\sim 200 \mathrm{fs}$, and, which is more important, at the same time probe these processes with the appropriate time resolution. The first approach to compare the LLB calculations 


\subsection{The Experimental Procedure}

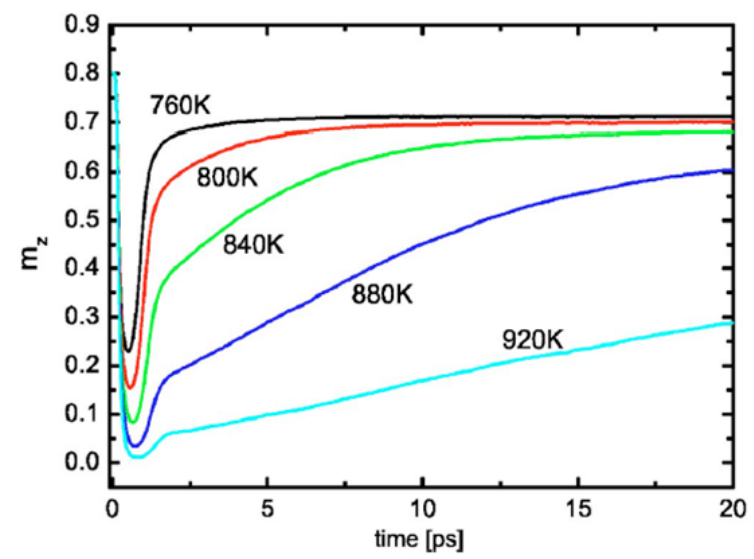

Figure 1.5: Magnetization dynamics after the heating with ultrashort laser pulses on a time scale up to $20 \mathrm{ps}$. Simulations of the LLB equation on $24 \mathrm{~nm}$ sized discretized cubes of nickel with $T_{C}=630 \mathrm{~K}$, taken from [5].

with laser induced magnetization dynamics was done in [5]. There the magnetization dynamics after heating a sample with a laser pulse was simulated by the LLB equation till up to 20 ps after excitation, by constantly increasing the pump fluence, for a cube with edge length $24 \mathrm{~nm}$, discretized in $2 \mathrm{~nm}$ cells. Electron temperatures of $T=920 \mathrm{~K}$, with $T_{C}=630 \mathrm{~K}$, were able to quench the magnetization into a paramagnetic state (see figure 1.5). The features, predicted in those simulations, can be observed in pump-probe magnetization dynamics experiments, and will be discussed in the next section. The first feature is the fast demagnetization on the $\sim 100 \mathrm{fs}$ time scale. The second feature is the slower recovery on the $\sim 10 \mathrm{ps}$ time scale. The higher the demagnetization of the system, the slower is also the recovery time, due to the decrease of exchange interactions at highest temperatures, which need to be recovered first, before the magnetization can relax into the equilibrium state, a consequence of the second (longitudinal) term of the LLB equation.

A more thorough study was performed in [6]. There the LLG equation was modeled to data obtained from all-optical pump-probe experiments for in-plane magnetized nickel thin films. The experimental data was obtained using the setup outlined in figure 1.4. There the sample is situated between two pole shoes of an electromagnet, and can be moved to be placed in the center, where the magnetic field is most homogeneous. The MOKE signal is measured by the probe beam, incident in a $35^{\circ}$ angle to the surface normal of the investigated sample. The signal is measured using a crossed polarizer configuration modulated by a photo-elastic modulator, PEM at $50 \mathrm{kHz}$. The pump beam hits the sample perpendicular to the film plane, and is modulated by a mechanical chopper at a frequency of $800 \mathrm{~Hz}$. Its pulses carry energies up to $3 \mu \mathrm{J} /$ Pulse, allowing fluences from $10 \mathrm{~mJ} / \mathrm{cm}^{2}$ up to $50 \mathrm{~mJ} / \mathrm{cm}^{2}$. The spot sizes of the probe and pump beam striking the sample surface are $\sim 30 \mu \mathrm{m}$ and $\sim 60 \mu \mathrm{m}$ respectively. The temporal delay $\Delta \tau$ between the pump and probe pulse at the sample is realized by a delay stage, changing the path length of the pump 
beam ${ }^{1}$. Magnetization dynamics (TRMOKE) is measured using the double modulation technique [39]. Reflectivity is measured using a single modulation scheme, simply recording the delay time dependent change in reflectivity, modulating only the pump beam. The laser pulses are generated in a self-built Ti:Sapphire oscillator and amplified by a Coherent RegA 9060 regenerative amplifier at a repetition rate of $250 \mathrm{kHz}$. A more detailed description of this setup is given in [21, 63].

The examined samples were prepared in a UHV chamber. Nickel was deposited using e-beam evaporation on Si (100) single crystal substrates [22]. Each film thickness is a different sample. A capping layer to prevent oxidation of the nickel was abandoned to minimize other contributions to the reflectivity signal, than the nickel's, and avoid non-local damping effects in the magnetization signal. Magnetization dynamics and reflectivity measurements were performed ex situ directly after sample preparation, before oxidation effects occurred and influenced the reflectivity properties.

Applying different laser pump fluences, allowed the electron temperature to increase up to above $T_{C}=631 \mathrm{~K}$ on a $15 \mathrm{~nm}$ nickel thin film. Maximum demagnetization from $10 \%$ to $40 \%$ was obtained. The data show that all demagnetization and recovery times increase with the pump fluence, and hence with the temperature. The increase of the recovery time with an increasing temperature and demagnetization, is a consequence of the second term in the LLB equation, as already stated above. The slowing down of the demagnetization is ascribed to the fact that during the laser pulse heating, the electron temperature changes much faster than $\tau_{\|} \sim 0.1-1 \mathrm{ps}$, therefore the spin system cannot follow the electron temperature, and the longitudinal relaxation is slowed down, causing an increase of the demagnetization time.

\subsubsection{The Two Temperature 2T and Three Temperature 3T Model}

The change of magnetization in the above derived LLB equation depends on two temperature dependent damping parameters (as described in section 1.2.1), $\alpha_{\|}$and $\alpha_{\perp}$, the effective field $\boldsymbol{H}_{\text {eff }}$ and the magnetization $\boldsymbol{m}$ itself. In order to solve this equation and determine the magnetization dynamics, $\boldsymbol{m}(t)$, the input of the electron temperature ${ }^{2} T_{e}$ is crucial, as all those input parameters depend on this temperature. Experimentally this is a major task, because there is no direct, time dependent access to the temperature.

There are two models that allow to disentangle the temperatures in systems heated with ultrashort laser pulses. The first one, developed in the 1970s [1], the two temperature model $2 \mathrm{~T}$, suggests an artificial separation of the electron system and the

\footnotetext{
${ }^{1}$ The theoretical descriptions of the dynamics are given as functions of time $t$. The experimental data is obtained as change of properties depending on the delay time $(\Delta \tau)$ of both laser pulses, where $\Delta \tau=0$ is the time, when both pulses have zero delay.

${ }^{2}$ In previous sections, this temperature has been represented by the variable $T$. A further distinction is needed, because later also the spin temperature $T_{s}$, and lattice temperature $T_{l}$ will be introduced.
} 


\subsection{The Experimental Procedure}

lattice and a treatment of both as separate heat baths interacting with each other. Both heat baths are ascribed a temperature and a heat capacity. The second one, is an extension of the 2T, the three temperature model 3T, suggested by Beaurepaire et al. [10] in 1996, after they first measured a demagnetization spectrum, using femtosecond laser pulses. In this model, a third heat bath is added, the spin system, enabling the description of the temporal evolution of magnetization.

Both models are experimentally accessible with the setup introduced in chapter 1.3.1. The only thing that changes is the detection scheme. For the $2 \mathrm{~T}$ model the time-resolved reflectivity is measured, it mirrors the evolution of the electron temperature after excitation with the pump pulse. For the 3T model, the time-resolved change in Kerr rotation is detected (TRMOKE). This scheme mirrors the state of magnetization $M$. The only experimental difference is the polarizer-analyzer configuration (see figure 1.4). The reflectivity measurement requires as much intensity of the reflected beam as possible, requiring a parallel polarizer-analyzer configuration, measuring $\Delta R(t)$. The TRMOKE measurement detects the change of the polarization of the reflected light, due to the change of magnetization after heating measuring the change in Kerr rotation $\Delta \theta(t)$. This requires the crossed polarizeranalyzer configuration. The probe beam passes through a polarizer in the parallel alignment to its polarization, before it is reflected at the sample. After the reflection, it passes through a polarizer rotated by $90^{\circ}$. When the polarization of the reflected light changes, a change in reflectivity proportional to the change of magnetization can be detected. This minimal adjustment between experiments enables consecutive measurements of both parameters, ensuring equal conditions, necessary for comparison.

\subsubsection{Determining the Spin Temperature via the 2T Model}

The electron temperature can be inferred from the reflectivity experiments assuming that $\Delta R(t) \sim T_{e}(t)$, by using the 2T model [54]. The photons carried by the laser pulse are absorbed by the electron system. However, the measured $\Delta R$ values are arbitrary and need to be calibrated, in order to obtain reliable temperature values. Although the experiments introduced in this thesis were carried out most thoroughly, the signal changing with the laser fluence can not be interpreted trivially. One issue that needs to be resolved is that the ratio between the pump and the probe beam is always 95:5. That means that increasing the pump fluence also increases the intensity of the probe beam and thus the intensity detected by the diode. Therefore the $\Delta R$ for the different pump fluences are not directly comparable. To determine the temperature, the same procedure as in [6] will be utilized.

In this procedure both the $2 \mathrm{~T}$ and the $3 \mathrm{~T}$ model have to be applied simultaneously. The $2 \mathrm{~T}$ model describes the heat evolution of the electron and the lattice system, 


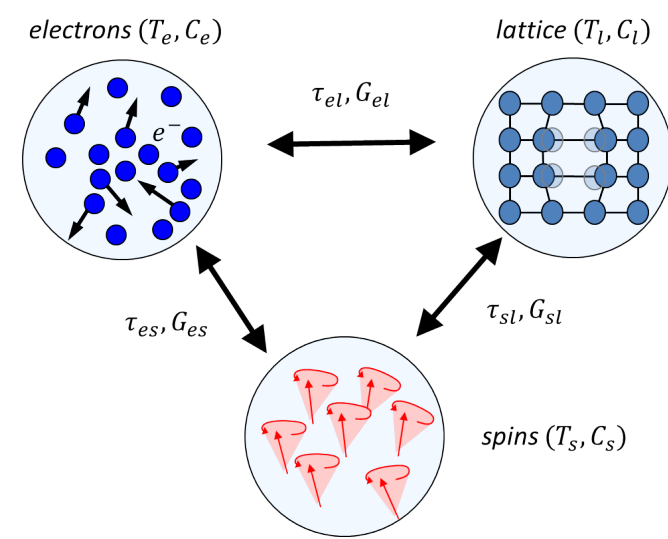

(a) The 3T Model artificially separates the electrons, spins and phonons into individual heat baths, ascribing each bath a temperature $T_{x}$ and a heat capacity $C_{x}$. The interaction between the heat baths is given by the heat mixing parameters $G_{e s}, G_{e l}, G_{s l}$ and determined by the relaxation times $\tau_{x y}$.

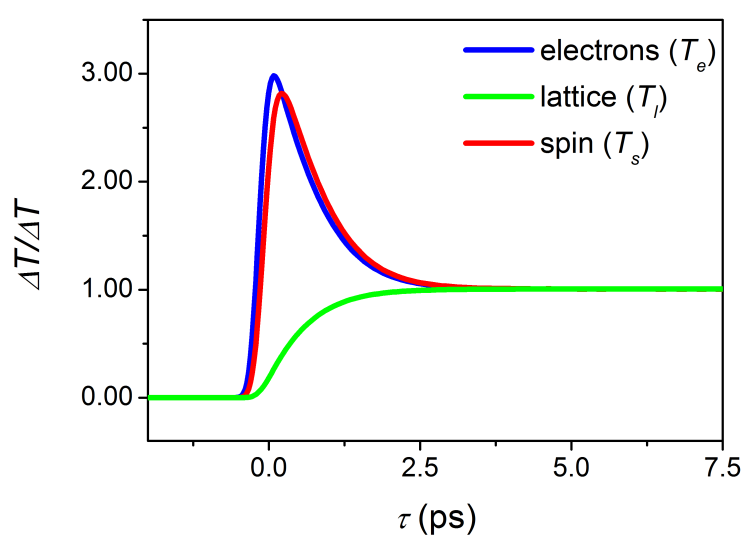

(b) Numerical solution to the differential equations from the $3 \mathrm{~T}$ model, showing the temporal evolution of, the electron $\left(T_{e}\right)$, the lattice $\left(T_{l}\right)$ and the spin $\left(T_{s}\right)$ temperatures in nickel.

Figure 1.6: Illustration of the $3 \mathrm{~T}$ model and the solution for the temperatures in nickel, taken from [47].

after ultra short heating, given by the set of two differential equations:

$$
\begin{aligned}
C_{e} \frac{d T_{e}}{d t} & =-G_{e l}\left(T_{e}-T_{l}\right)+P(t)-C_{e} \frac{\left(T_{e}-T_{\text {room }}\right)}{\tau_{t h}}, \\
C_{l} \frac{d T_{l}}{d t} & =G_{e l}\left(T_{e}-T_{l}\right) .
\end{aligned}
$$

This set of equations can be solved numerically [19], and analytically [45]. The analytical solution requires simplifications, like neglecting the heat diffusion $C_{e} \frac{\left(T_{e}-T_{\text {room }}\right)}{\tau_{t h}}$, where $\tau_{t h} \approx 50 \mathrm{ps}$, and assuming a $\delta$-function for the laser pulse shape.

The $3 \mathrm{~T}$ model includes a third differential equation assigning a third heat bath for the spin system, and describing its temporal heat evolution:

$$
C_{s} \frac{d T_{s}}{d t}=G_{e s}\left(T_{e}-T_{s}\right)+G_{l s}\left(T_{l}-T_{s}\right) .
$$

Solutions to the 3T model are also given in [19] a numerical, and in [45] an analytical solution. In figure 1.6 taken from [47], a graphic illustration of the 3T model (a), and graphs describing the temporal temperature evolution (b) are shown. Figure 1.6(b) shows that the spin temperature $T_{s}$ almost immediately follows the electron temperature $T_{e}$, both reach a maximum and relax to an elevated equilibrium temperature. The lattice temperature slowly increases to the elevated equilibrium temperature, reaching it as a maximum. This behavior also mirrors the heat capacities of the 


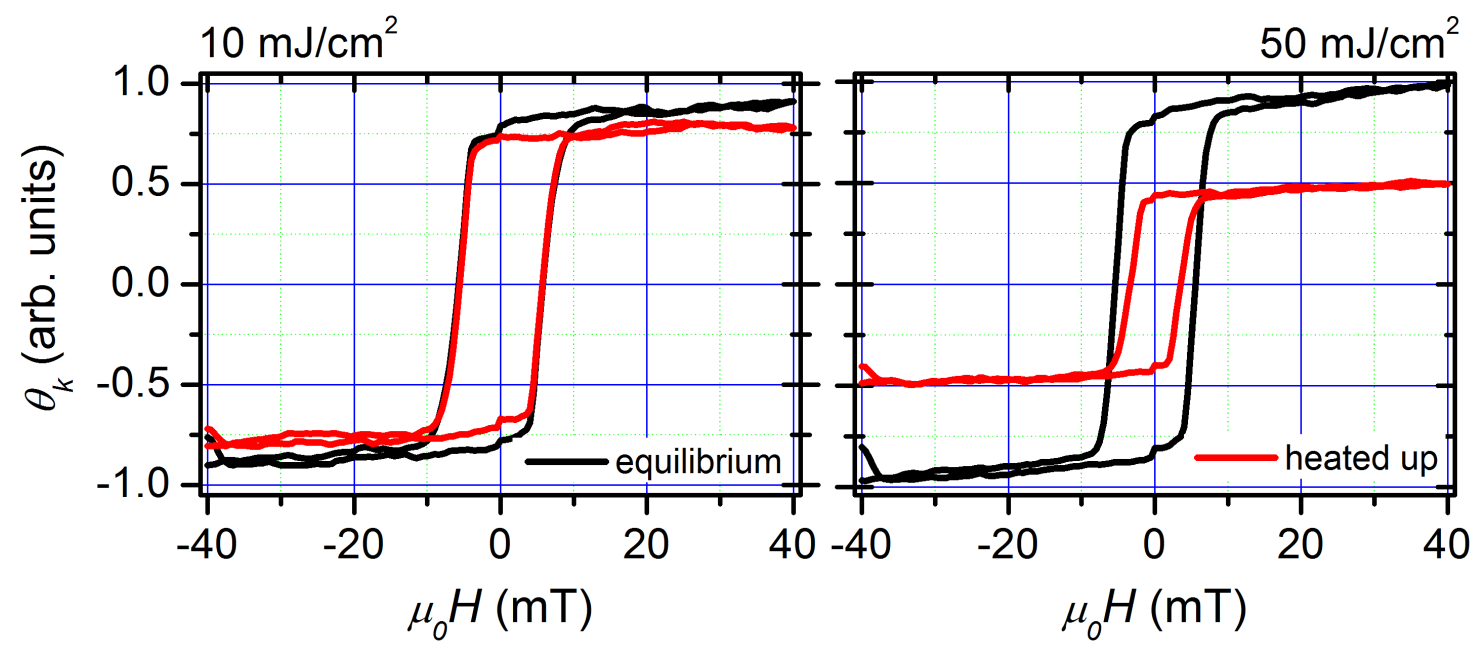

Figure 1.7: Hysteresis loops of the $10 \mathrm{~nm}$ nickel film measured at $\Delta \tau=0 \mathrm{ps}$. One with a blocked pump beam, representing the equilibrium state at $300 \mathrm{~K}$ (black). The other with the pump beam heating the sample, (red). The pump fluence of $10 \mathrm{~mJ} / \mathrm{cm}^{2}$, demagnetizing the sample only slightly (left), and at $50 \mathrm{~mJ} \mathrm{~cm}^{2}$ around $40 \%$.

baths. The energy absorbed by the electrons influences the lattice temperature only slightly and much slower, because of the much higher heat capacity. Although the coupling parameter $\lambda=0.045$ is rather small, indicating a weak coupling of the spin system to the electron bath, $C_{s} \ll C_{e}$, and spin temperature follows the electron temperature immediately, resulting in a fast demagnetization. Therefore systems exhibiting a fast demagnetization are considered strongly spin-electron coupled, in contrast to systems, where the spin temperature increase and demagnetization proceeds rather slowly. After $\sim 7.5 \mathrm{ps}$ the temperatures of all three heat baths reach the same elevated temperature.

The electron temperatures $T_{e}$ and the spin temperatures $T_{s}$ are determined from the experimental magnetization and reflectivity dynamics data as follows. The recorded TRMOKE data are given in arbitrary units of the change of the Kerr angle with respect to the Kerr angle at $300 \mathrm{~K}$ in the saturated equilibrium state $\Delta \theta_{k} / \theta_{k, 300 \mathrm{~K}}(\Delta \tau)$, at a given time $\Delta \tau$ before and after excitation. These data can be calibrated to obtain the change of magnetization $\Delta M / M_{300 \mathrm{~K}}(\Delta \tau)$, using stationary hysteresis measurements. For this purpose, at each fluence two hysteresis loops are recorded, shown in figure 1.7 for a low $10 \mathrm{~mJ} / \mathrm{cm}^{2}$ (left) and a high $50 \mathrm{~mJ} / \mathrm{cm}^{2}$ fluence (right). One hysteresis loop, when the pump beam is blocked, mirroring the Kerr angle in the equilibrium state at $300 \mathrm{~K}$ (black line), and the other, when the pump beam is perturbing the sample at zero delay $\Delta \tau=0$ ps (red line), mirroring the Kerr signal in the excited state. Then, from the relative quenching of the Kerr angle determined from both hysteresis loops, the percentage of demagnetization at the measured delay is calculated, assuming $\theta_{k} \sim M$. Finally, setting the 


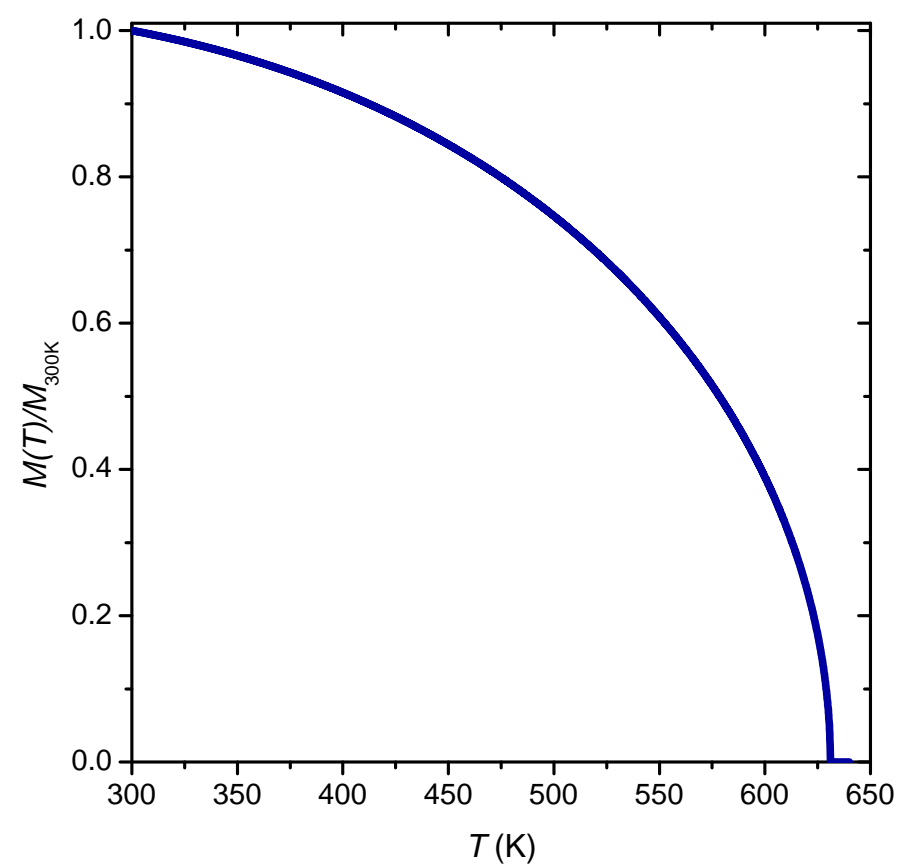

Figure 1.8: Solution to the Brillouin function for $T_{C}(\mathrm{Ni})=631 \mathrm{~K}$, normalized to the equilibrium magnetization at room temperature.

recorded TRMOKE signal before excitation to $\Delta M / M_{300 \mathrm{~K}}(\Delta \tau<0)=1$, and knowing the demagnetization value from the hysteresis analysis, the TRMOKE curves are calibrated, showing the state of magnetization $\Delta M / M_{300 \mathrm{~K}}$ at each point of time delay $\Delta \tau$. Now, the spin temperature can be determined from the Curie-Weiss law expressed by the Brillouin function for spin $S=\frac{1}{2}$, for any given $\Delta \tau$ :

$$
M^{*}=\frac{M(T)}{M(0 \mathrm{~K})}=\tanh \left(\frac{T_{C}}{T} M^{*}\right) .
$$

The solution to equation 1.16 is obtained using a root solver in Scilab, for $T_{C}(\mathrm{Ni})=$ $631 \mathrm{~K}$, given in figure 1.8 . This curve is normalized to the equilibrium magnetization at room temperature. In the derivation of the LLB equation the fluctuations are described by Langevin fields, as well as in [5, 6], there also the Langevin function was used to determine the temperature. These models are applied for the classical spin, therefore the Langevin approach seems appropriate. In the future however, the LLB equation will be used in its extended version for $S=\frac{1}{2}$, when modeling the dynamics of transition metals [3]. Therefore, here the Brillouin function for $S=\frac{1}{2}$ is implemented.

According to the $3 \mathrm{~T}$ model, $7.5 \mathrm{ps}$ after excitation, all three systems are at an elevated equilibrium temperature. To assure the equilibrium state even at higher fluences, a later time $\Delta \tau=20 \mathrm{ps}$ after excitation is chosen for calibration. At this time point, the spin temperature $T_{s}$ is extracted from the magnetization using the Brillouin function. Because the electron temperature at 20 ps after excitation equals 


\subsection{The Experimental Procedure}

the spin temperature $\left(T_{e}=T_{s}\right)$, and knowing that the electron temperature before excitation is at $300 \mathrm{~K}$, the time-resolved reflectivity curves $\Delta R(\Delta \tau)$ are calibrated to obtain the time-resolved electron temperature $T_{e}(\Delta \tau)$.

Assuming the specific heat capacities, $C_{e}=\gamma_{e} T_{e}\left(\gamma_{e}=3 \cdot 10^{3} \mathrm{Jm}^{-3} \mathrm{~T}^{-2}\right)$, and $C_{l}=C(300 \mathrm{~K})-C_{e}(300 \mathrm{~K})=3.1 \cdot 10^{6} \mathrm{Jm}^{-3} \mathrm{~K}^{-1},\left(C(300 \mathrm{~K})=4 \cdot 10^{6} \mathrm{Jm}^{-3} \mathrm{~K}^{-1}\right)$ for the electrons and the lattice, the electron-phonon coupling constant $G_{e l}$, describing the amount of energy exchange between the electrons and the lattice, is determined to $G_{e l} \approx 1 \cdot 10^{17} \mathrm{Wm}^{-3} \mathrm{~K}^{-1}$. The heat diffusion time is $\tau_{t h} \approx 50 \mathrm{ps}$. Both values are consistent with values obtained earlier [6].

The critical parameter in this calculation is the Gaussian power source term $P(t)$, which determines the amount of pump-pulse power density deposited at the sample. While $P(t)$ can be determined from the laser power $(100-700 \mathrm{~mW})$, the repetition rate $(\sim 250 \mathrm{kHz})$ and the spot size at the sample $(\sim 60 \mu \mathrm{m})$, it is rather difficult to calculate, how much energy is actually absorbed by the Ni film, and contributing to the temperature rise. In addition, the $\mathrm{Ni}$ film is deposited on a $\mathrm{Si}$ substrate, which, depending on the Ni film thickness, causes reflections on the interface and a different absorption by the Si substrate itself. All these experimental circumstances and the penetration depth of the laser light into the sample require a thoughtful consideration, when calculating the absorbed power density.

The time-resolved magnetization $\Delta M / M_{300 \mathrm{~K}}(\Delta \tau)$ and the time-resolved electron temperatures $T_{e}(\Delta \tau)$ extracted from the experimental data, are presented in appendix $\mathrm{B}$. The $T_{e}(\Delta \tau)$ data shows oscillations on top of the relaxation signal. Their amplitude increases with the pump fluence, and decreasing nickel film thickness. These oscillations are ascribed to stress waves which stem from the stress on the interface between the nickel film and the silicon substrate. These oscillations do not influence the electron temperature, and will not be further analyzed in this work. For the analysis within the thermal model (LLB equation), the $2 \mathrm{~T}$ model is fitted to the calibrated $T_{e}(\Delta \tau)$ curves, see figure 1.13 in section 1.4 .3 . The $2 \mathrm{~T}$ model does not take the oscillations into account, therefore the fitted functions provide simple exponentially decaying curves that can be easier implemented into the LLB simulations. 


\subsection{Experimental Results}

\subsubsection{Penetration Depth of Light into Metals}

The penetration depth of light into a metal can be calculated according to optics textbook knowledge, e.g. Hecht [29]. The wave equation (Telegrapher's equation) derived from Maxwell's equations is:

$$
\frac{\partial^{2} \vec{E}}{\partial x^{2}}+\frac{\partial^{2} \vec{E}}{\partial y^{2}}+\frac{\partial^{2} \vec{E}}{\partial z^{2}}-\mu_{0} \epsilon \frac{\partial^{2} \vec{E}}{\partial t^{2}}-\mu_{0} \sigma \frac{\partial \vec{E}}{\partial t}=0 .
$$

With $\epsilon$ the permittivity and $\sigma$ the conductivity, determining the behavior of an electromagnetic wave. The last term on the left hand side is the first partial derivative and indicates a damping. For an electromagnetic wave, entering a metal with a complex refraction index $n_{\mathrm{C}}=n_{\mathrm{R}}-i n_{\mathrm{I}}$, one solution to the wave equation is:

$$
\vec{E}=\vec{E}_{0} \cos \omega\left(t-n_{\mathrm{R}} z / c\right) \exp \left(-\omega n_{\mathrm{I}} z / c\right) .
$$

This equation describes a wave propagating in $z$-direction with the velocity $c / n_{\mathrm{R}}$. Electromagnetic waves (light waves) with an alternating $\vec{E}$ vector, which enter a medium with the conductivity $\sigma$, cause a voltage, which induces a current. Due to the resistivity $\sim 1 / \sigma$ of the medium, the current is converted into heat, or light is absorbed. The intensity of the light wave is defined as $I=\varepsilon_{0} c E^{2}$ or more general, $I \sim|E|^{2}$. Therefore, the light wave entering the medium is damped by the damping coefficient $1 / \Lambda_{\mathrm{opt}}=2 \omega n_{\mathrm{I}} / c$. The reciprocal value $\Lambda_{\mathrm{opt}}=\lambda /\left(4 \pi n_{\mathrm{I}}\right)$ gives the penetration depth of the light wave.

Considering the central wavelength of $\lambda=800 \mathrm{~nm}$ used in the presented experiments, and the corresponding refractive index of nickel $n_{\mathrm{C}}=2.48-i 4.38$, taken from [49, p.313], gives a penetration depth of the laser light into nickel of $\Lambda_{\text {opt }}=14.5 \mathrm{~nm}$.

\subsubsection{Absorbed Laser Power Density}

Considering a sample, which spacial expansion is described by the vector $\vec{r}=$ $(x, y, z)$, the parameter $P(\vec{r}, t)$ in the relation:

$$
P(\vec{r}, t)=\frac{\alpha_{a b s} \cdot F(\vec{r}, t) \cdot e^{-z / \Lambda_{\mathrm{opt}}}}{\Lambda_{\mathrm{opt}} \cdot\left(1-e^{-d / \Lambda_{\mathrm{opt}}}\right)}
$$

describes the amount of the absorbed power density, when being illuminated by a light pulse with the time dependent pump fluence $F(\vec{r}, t)$ from a laser beam pointing in $z$ direction. The focus on the data analyzed below lies on the time of maximum demagnetization, which is usually reached within around 300 fs after the pump pulse has been absorbed by the sample. The pulse length is $80 \mathrm{fs}$, and the time between two pulses is $4 \mu \mathrm{s}$, assuring enough time for temperature relaxation into the initial state between pulses. Therefore, the first part of the analysis will focus on the total energy deposited by a single pulse, neglecting its temporal expansion. Further, 


\subsection{Experimental Results}

heat diffusion will be neglected in the calculations, because it becomes important only $50 \mathrm{ps}$ after excitation. The spacial expansion of $F$ is already considered by the measurement of the beam profile, $60 \mu \mathrm{m}$ in diameter. The factor $\alpha_{a b s}$ is the absorption coefficient given by $\alpha_{a b s}=(1-R-\hat{T})$. With $R$ being the amount of light reflected by the material, and $\hat{T}$ the amount of light transmitted through the material. Considering an angle of incidence of the pump beam around $0^{\circ}$ to the surface normal, yields in $R=0.68301$ for all nickel layers. This can be obtained by applying Fresnel's equations for the reflection of light from materials with a complex refractive index $n_{C}$ :

$$
R=\frac{\left(n_{R}-1\right)^{2}+n_{I}^{2}}{\left(n_{R}+1\right)^{2}+n_{I}^{2}}
$$

The denominator in equation 1.17 expresses the energy conservation by normalizing the expression. This yields for a sample of the thickness $d$ :

$$
\int_{0}^{d} P(\vec{r}) d z=\alpha_{a b s} F(\vec{r}) .
$$

The factor $\alpha_{a b s}$ takes into account the energy that is not used for heating the investigated material due to reflection and transmission. The reflected part can be calculated easily, as shown in equation 1.18, and checked experimentally, by measuring the intensity of the reflected beam. It appears to be more challenging to estimate the transmission through the layer, especially for the thin nickel layers, with $d<\Lambda_{\text {opt }}=14.5 \mathrm{~nm}$. On one hand, the calculation, to be precise, should take care of the effects at the interface between the investigated metal layer, and the substrate, leading to complicated calculations. On the other hand, the transmitted light absorbed by the substrate cannot be measured experimentally. This leaves $\alpha_{a b s}$ to be the most critical parameter in the estimation of the absorbed power density, contributing the largest error to the temperature calculated within the $2 \mathrm{~T}$ model. A too low estimated $\hat{T}$ leads to a higher $\alpha_{a b s}$, and thus to an overestimation of the peak temperature, and vice versa.

The transmission $\hat{T}$ in the absorption factor will be calculated using the exponential decay of the intensity:

$$
\alpha_{a b s}=(1-R) \cdot \frac{1}{\Lambda_{\mathrm{opt}}} \cdot \int_{0}^{d} e^{-z / \Lambda_{\mathrm{opt}}} d z
$$

using $\Lambda_{\text {opt }}$ from section 1.4.1. The factor $1-R$ takes care of the part reflected from the layer, so that the amount absorbed can be estimated by the integral. Considering a refractive index of nickel, as given in section 1.4.1 and the refractive index of crystalline silicon $n=3.69$ for the wavelength $\lambda=800 \mathrm{~nm}$, the reflected amount of light at the interface between nickel and silicon according to Fresnel's equations is around $4 \%$. That means, the contribution of reflections at the interface between the nickel film and the substrate, contributing to electron excitations can be neglected in this analysis. 


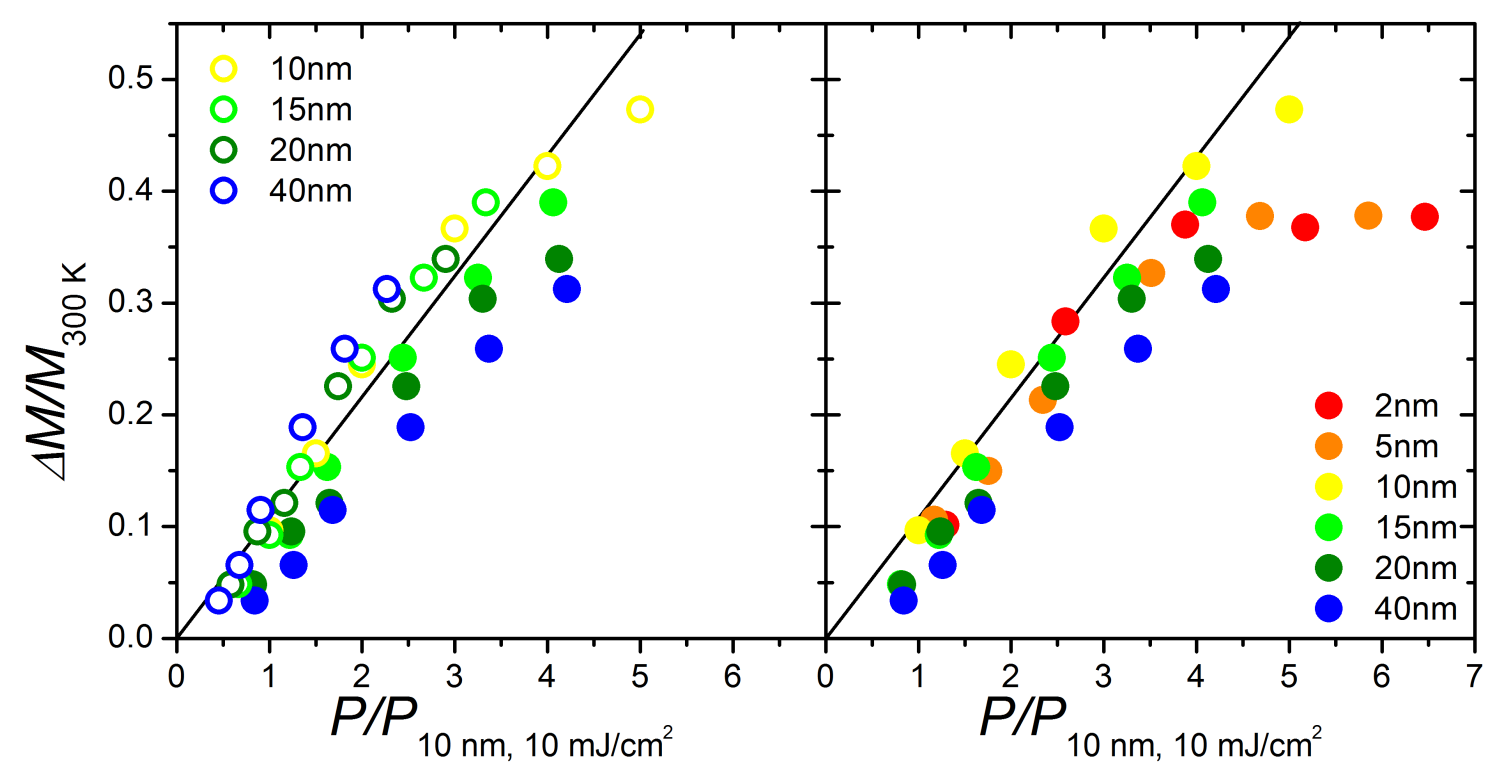

Figure 1.9: Demagnetization $\Delta M / M-300 \mathrm{~K}$ plotted against the applied pump fluences rescaled to the per nickel layer absorbed power density $P / P_{10 \mathrm{~nm}, 10 \mathrm{~mJ} / \mathrm{cm}^{2}}$. The open circles represent the calculation assuming the same absorption coefficient $\alpha_{a b s}$ for all nickel thicknesses, whereas the closed circles represent the calculations including thickness dependent absorption coefficients, as described in equation 1.19 . The lines are guides to the eye.

Figure 1.9 shows the applied pump fluences, calculated to the absorbed power density $P$ and rescaled to the power density absorbed by the $10 \mathrm{~nm}$ nickel film perturbed by $10 \mathrm{~mJ} / \mathrm{cm}^{2}, P_{0}$. The calculation details are given in the appendix A.

The data calculated neglecting the thickness dependence of the transmission coefficient $\hat{T}$ show a linear relation to the measured demagnetization (left open circles), for nickel films of thickness $d \geq 10 \mathrm{~nm}$. Calculating the per layer absorbed power density for the two thinnest films in the analysis, under the assumption of a thickness independent $\hat{T}$, yields an energy density that is up to five times as high for the $2 \mathrm{~nm}$ thick film, as for the others. Surprisingly, those two thinnest nickel layers, $d=2 \mathrm{~nm}$ and $d=5 \mathrm{~nm}$ do not show higher maximum demagnetization rates with increasing pump fluence, than the thicker films.

Taking into account the thickness dependence of the transmission factors for different nickel films, the per layer absorbed power density remains constant for the three thickest nickel films for each fluence. There are no significant changes, although for these films the thickness difference is larger, than for the three thinnest films. At the same time the demagnetization $\Delta M / M_{300 \mathrm{~K}}$ increases with decreasing film thickness. Furthermore, the relation between the per nickel layer absorbed energy and the demagnetization stays linear for each of the three thickest films. The data for the three thinnest layers on the other hand, show an increasing per layer absorbed energy with decreasing film thickness not only in comparison to the three 


\subsection{Experimental Results}

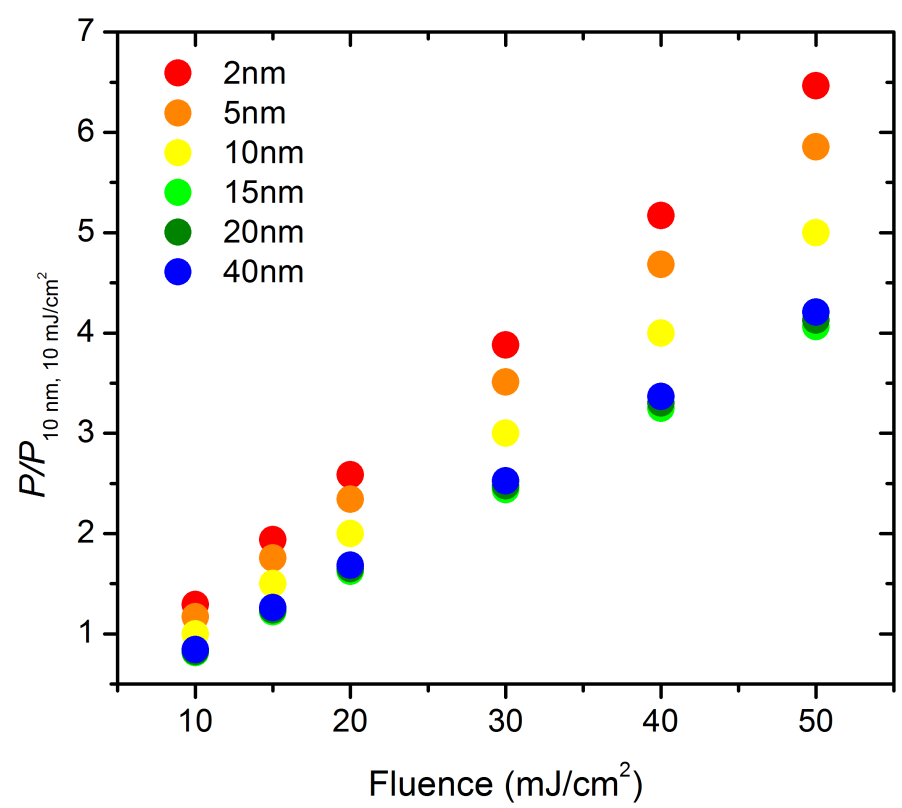

Figure 1.10: The per layer absorbed power density remains constant at all fluences for nickel films thicker than the penetration depth $\Lambda_{\text {opt }}=14.5 \mathrm{~nm}$. In nickel films thinner than $\Lambda_{\text {opt }}$, the per layer absorbed power density increases with decreasing film thickness.

thickest layers, but also between each other. That means, the $2 \mathrm{~nm}$ thick nickel film absorbs more energy per layer for each fluence, than the $5 \mathrm{~nm}$ thick film and the $10 \mathrm{~nm}$ film. However, the demagnetization saturates at around $40 \%$ and does not increase further with increasing pump fluence.

For clarity, figure 1.10 shows the relation between the pump fluence and the per layer absorbed energy normalized to the energy absorbed by the $10 \mathrm{~nm}$ film pumped by a $10 \mathrm{~mJ} / \mathrm{cm}^{2}$ laser fluence. The relation for this fluence range and film thickness is linear, as expected from the formulas given in the appendix A. The deviation from linearity will not occur for thicker samples, because the transmission, as defined above does not change significantly for films thicker than $\Lambda_{\text {opt }}$, and also the term $\exp \left(-d_{\mathrm{Ni}} / \Lambda_{\text {opt }}\right)$ converges to 0 with a decreasing slope. Therefore, the thicker films, starting with $d_{\mathrm{Ni}}=15 \mathrm{~nm}$ absorb approximately the same amount of energy per layer at each fluence. The thinner films on the other hand absorb a higher amount of energy per layer at each fluence, with the highest amount being absorbed by the thinnest nickel film. This suggests that for all nickel films, the maximum electron temperature constantly increases with the pump fluence, and the thinner films reach higher electron temperatures. 


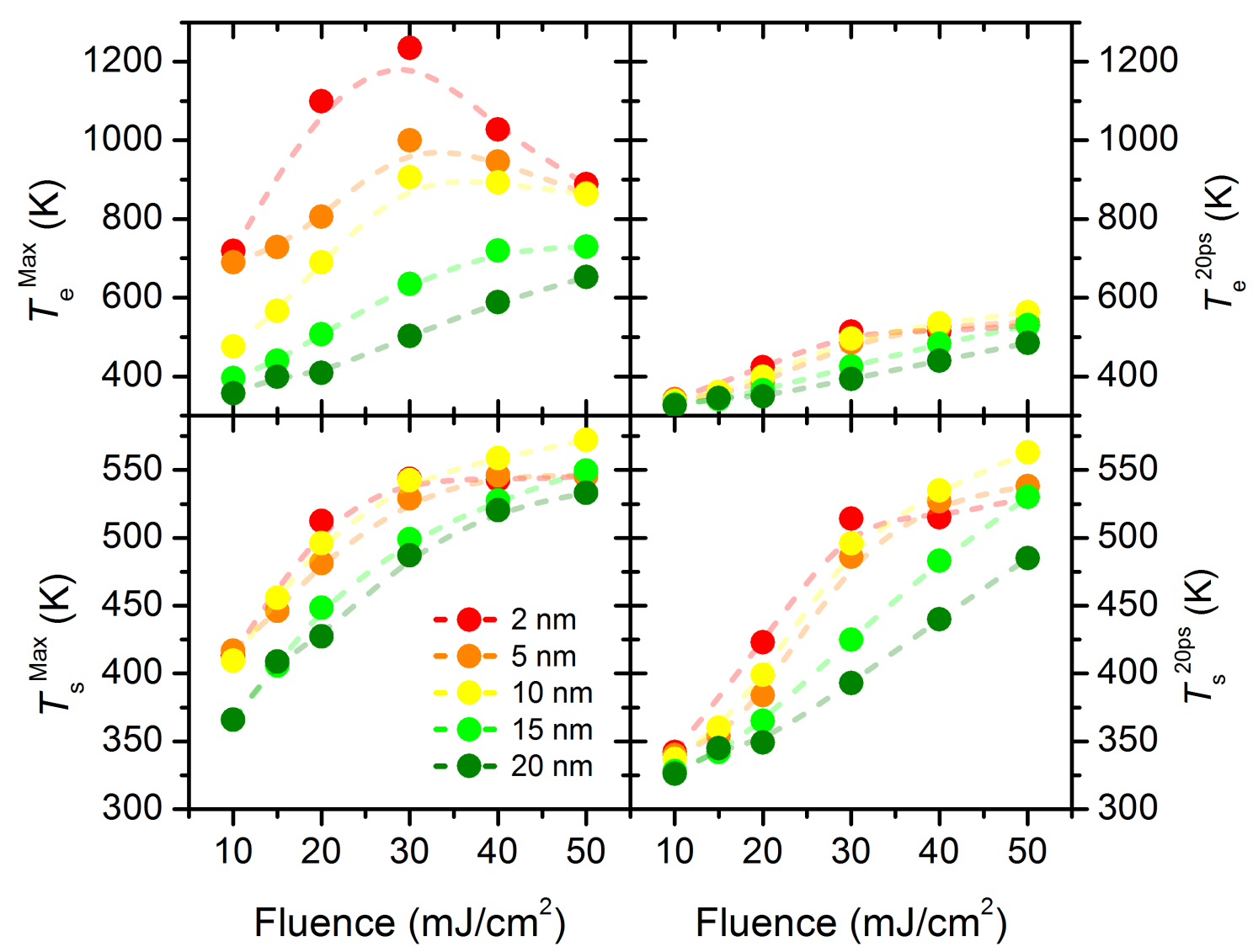

Figure 1.11: Peak electron (top) and spin (bottom) temperatures (left) and the corresponding temperatures $20 \mathrm{ps}$ after excitation (right) plotted against the applied pump fluence, for the different nickel film thicknesses.

\subsubsection{Reflectivity Data and Electron Temperatures}

Figure 1.11 shows the peak electron $T_{e}^{\mathrm{Max}}$ and peak spin temperatures $T_{s}^{\mathrm{Max}}$ and the corresponding temperatures $20 \mathrm{ps}$ after excitation $T_{e}^{20 \mathrm{ps}}$, and $T_{s}^{20 \mathrm{ps}}$, plotted against the applied pump fluence, for the different nickel film thicknesses. All temperatures are extracted from the experimental reflectivity and magnetization dynamics data shown in appendix B, calibrated using the procedure described in section 1.3.3. The data show, especially for the thinner $2 \mathrm{~nm}, 5 \mathrm{~nm}$ and $10 \mathrm{~nm}$ nickel films that the electron temperature rises far above $T_{C}$. For these films, the peak temperature also reaches a maximum at a fluence around $30 \mathrm{~mJ} / \mathrm{cm}^{2}$, with the highest value for the thinnest film, exceeding $1200 \mathrm{~K}$. For higher fluences, the electron peak temperatures converge to values slightly below $900 \mathrm{~K}$. This shows a contradiction to the assumption from the calculations in the previous section, where the electron temperature should constantly increase with the pump fluences. The peak electron temperatures for the films thicker than the penetrations depth, $\Lambda_{\text {opt }}$, constantly increase with the pump fluence, not exceeding $730 \mathrm{~K}$ for the highest fluence. This behavior is in agreement with the suggestions made in the previous section. Despite these appar- 


\subsection{Experimental Results}

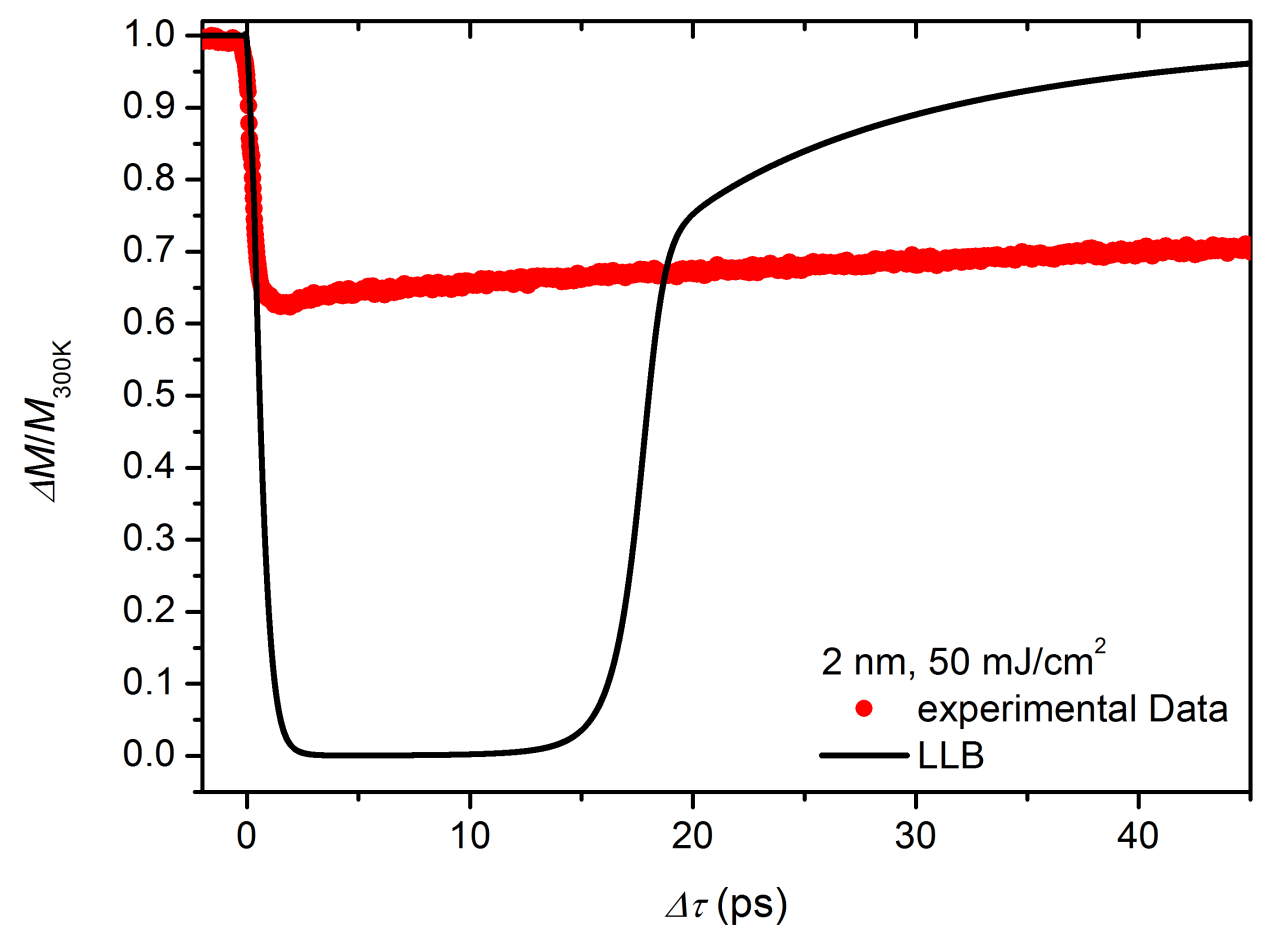

Figure 1.12: Magnetization dynamics of a $2 \mathrm{~nm}$ nickel film, excited with a pump fluence of $50 \mathrm{~mJ} / \mathrm{cm}^{2}$, modeled using the LLB equation, using electron temperatures from the reflectivity data in appendix $\mathrm{B}$ as input by Atxitia et al. [4. For comparison, the experimental data of the magnetization dynamics for the same film is shown (red dots).

ent high electron temperatures, occurring in the thinnest nickel films, the peak spin temperatures do not exceed $T_{C}$, even for the highest fluences. After 20 ps both the electron and the spin temperatures decrease below $T_{C}$ while the temperature drop of the spin system always lies below $100 \mathrm{~K}$, the electron temperature drop at this time scale for the three thinnest films lies above $100 \mathrm{~K}$, and reaches even $700 \mathrm{~K}$ for the thinnest film at $30 \mathrm{~mJ} / \mathrm{cm}^{2}$. The electron temperature drop from the peak temperature to the temperature at $20 \mathrm{ps}$ after excitation is proportional to the maximum temperature $T_{e}^{\mathrm{Max}}$. All electron peak temperatures in the presented experimental data are reached within $\sim 0.5$ ps after excitation.

According to the thermal model, due to the second order transition from the ferromagnetic state to the paramagnetic state around Curie temperature, the transverse relaxation processes determining $\tau_{\perp}$ are decreased, and the relaxation processes determining $\tau_{\|}$gain importance. That means that the critical slowing down, when the electron temperature changes faster than $\tau_{\|}$, and the spin system cannot follow the temperature, leads to a slower demagnetization, and if the electron temperature reaches its peak values and relaxes fast enough, to a saturation in the demagnetization. In order to reach a maximum demagnetization that corresponds to a temperature below $T_{C}$, also the electron temperature needs to decrease to a value 
below $T_{C}$ within the first $1 \mathrm{ps}$ after excitation. This behavior is directly visible from the reflectivity data, for the nickel films thicker than $\Lambda_{\text {opt }}$. The electron temperature increases for high pump fluences even above $T_{C}$, but decreases below $T_{C}$ within the first picosecond. The maximum demagnetization of these films does not exceed $\sim 40 \%$, but constantly increases with the pump fluence. Here the maximum demagnetization saturation point is not reached. This seems to be different for the thinner nickel films. In spite of the apparent high electron temperature, and a relaxation below $T_{C}$ within $10 \mathrm{ps}$, after the maximum has been reached, especially the $2 \mathrm{~nm}$ and the $5 \mathrm{~nm}$ film show a demagnetization saturation around $40 \%$. Magnetization dynamics data simulated using the LLB equation, employing electron temperatures extracted from the reflectivity data for the $2 \mathrm{~nm}$ film perturbed by the highest pump fluence $\left(50 \mathrm{~mJ} / \mathrm{cm}^{2}\right)$, shows a demagnetization into the paramagnetic state until up to around 15 ps after excitation. Figure 1.12 shows the data simulated by Atxitia et al. 4] and the experimental data for comparison. The fast recovery after $15 \mathrm{ps}$ in the simulated data is an artifact of the macrospin model, as discussed in [37] on spin dynamics of FePt films.

This disagreement with the experimental data shows that the electron temperatures extracted via the $2 \mathrm{~T}$ model from the reflectivity data, for nickel films thinner than the optical penetration depth $\Lambda_{\mathrm{opt}}$, are obviously overestimated. That means, the absorption coefficient for these optically thin films need a further thorough reconsideration. Apparently a thickness dependent absorption, as input into the $2 \mathrm{~T}$ model still delivers exaggerated electron peak temperatures. The discrepancy between the measured reflectivity data and the actual lower electron peak temperatures can be ascribed to the reflectivity contribution from the Si substrate. This contribution increases with decreasing nickel film thickness.

The data of the extracted electron temperatures in appendix $B$ show that after excitation, in general the relaxations are taking place on two time scales, and that the amplitude of the first relaxation decreases with the pump fluence, and decreasing film thickness. This circumstance is illustrated in figure 1.13 for the thinnest, the $2 \mathrm{~nm}$ nickel film, and the $20 \mathrm{~nm}$ nickel film both measured at the highest $\left(50 \mathrm{~mJ} / \mathrm{cm}^{2}\right)$ pump fluence. The $20 \mathrm{~nm}$ nickel film data clearly shows a fast relaxation on the time scale $\tau_{\mathrm{E}_{1}} \approx 0.5 \mathrm{ps}$, highlighted by the gray background. The duration of this relaxation does not change significantly with the pump fluence, or the film thickness. However, the amplitude of this relaxation decreases gradually, and is not distinguishable in the data of the two thinnest films at fluences $F \geq 40 \mathrm{~mJ} / \mathrm{cm}^{2}$. These data only show the second relaxation process. Following the first relaxation, there is a second, slower relaxation $\tau_{\mathrm{E}_{2}}$, which is film thickness dependent. The second relaxation is the fastest for the two thinnest nickel films in the analysis. The relaxation time lies slightly below $\tau_{\mathrm{E}_{2}}(2 \mathrm{~nm}, 5 \mathrm{~nm})<20 \mathrm{ps}$. With increasing film thickness, this relaxation slows down. The $10 \mathrm{~nm}$ nickel film, exhibits $\tau_{\mathrm{E}_{2}}(10 \mathrm{~nm})>25 \mathrm{ps}$, and the $20 \mathrm{~nm}$ nickel film shows already a second relaxation that is around $50 \mathrm{ps}$.

The constant value of $\tau_{E_{1}} \approx 0.5 \mathrm{ps}$ indicates the thermalization process due to electron-electron scattering [53] in the first relaxation process. The decreasing am- 


\subsection{Experimental Results}

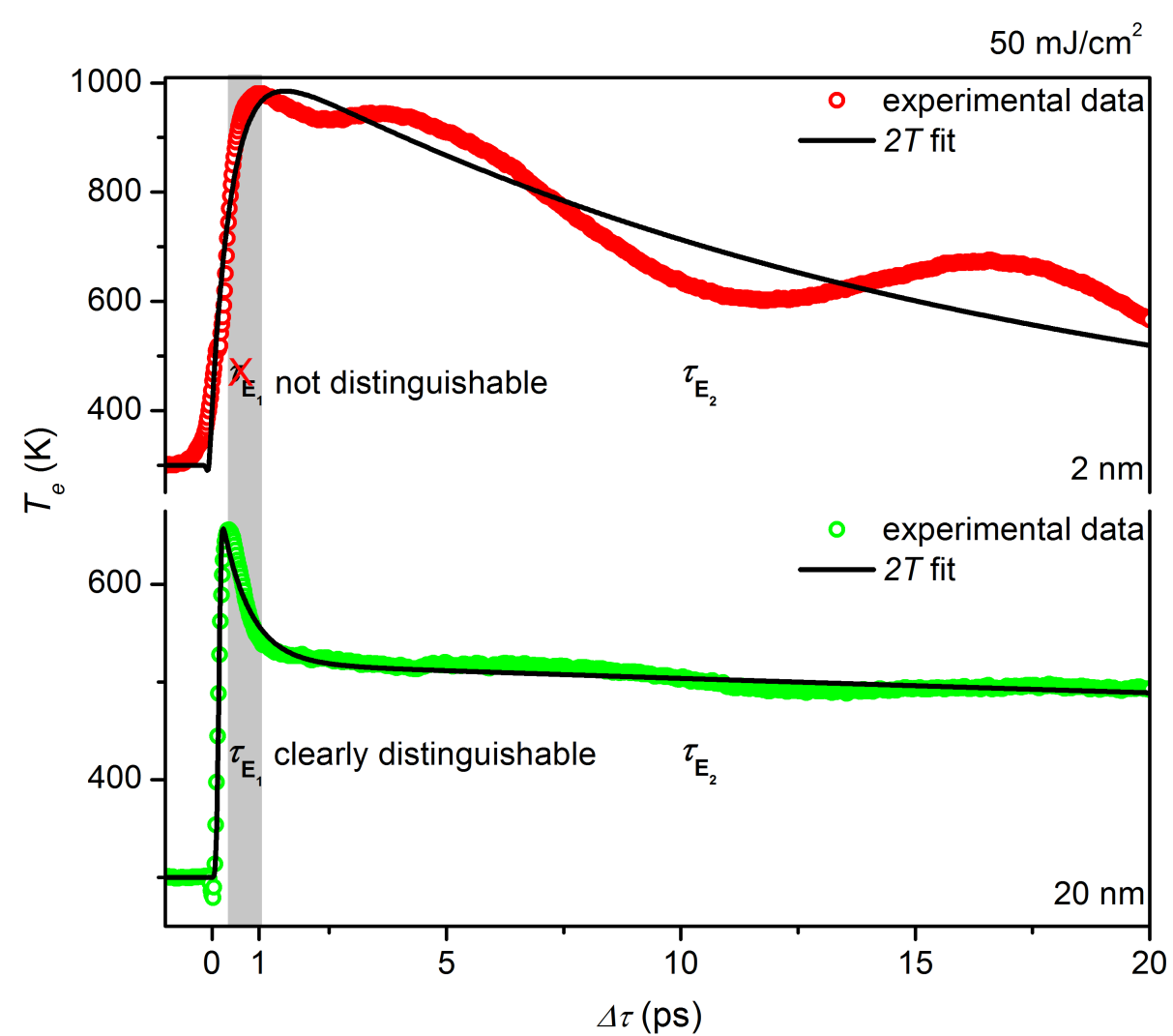

Figure 1.13: $T_{e}(\Delta \tau)$ curves at $50 \mathrm{~mJ} / \mathrm{cm}^{2}$ pump fluence for the thinnest, $2 \mathrm{~nm}$ and the $20 \mathrm{~nm}$ nickel films (open circles) and the corresponding $2 \mathrm{~T}$ fits (black lines). The $20 \mathrm{~nm}$ film electron temperature clearly shows a first relaxation $\left(\tau_{E_{1}}<\right.$ $1 \mathrm{ps})$ and a second relaxation $\left(\tau_{E_{2}} \approx 50 \mathrm{ps}\right)$, while the $2 \mathrm{~nm}$ film electron temperature only exhibits $\tau_{E_{2}}>20 \mathrm{ps}$.

plitude with increasing fluence shows that the electrons thermalize at higher temperatures, when the heating is stronger. The second relaxation is ascribed to electronphonon scattering, where heat is passed on from the electrons to the lattice.

The vanishing of the first relaxation process in the data for the two thinnest nickel films at fluences $F \geq 40 \mathrm{~mJ} / \mathrm{cm}^{2}$ does not mean there is no thermalization of the electrons. In the data for the films much thinner than the laser light penetration depth, there is a strong contribution of the silicon substrate to the reflectivity signal. In silicon, the density of the excited electrons is lower than in nickel, therefore the electron-electron scattering processes are not as pronounced in the reflectivity data, and only the second relaxation is distinguishable. Besides that, the contribution of the silicon in the reflectivity signal shows a faster relaxation of the electron-phonon scattering processes. This contribution in the measured signal is the reason for the overestimation of the maximum electron temperatures for the thinnest nickel films. Even though the electrons, the spins and the lattice in the nickel films can be considered at equilibrium temperature 20 ps after excitation, which is the point chosen for calibration of temperature, since the absolute values are obtained via the mag- 

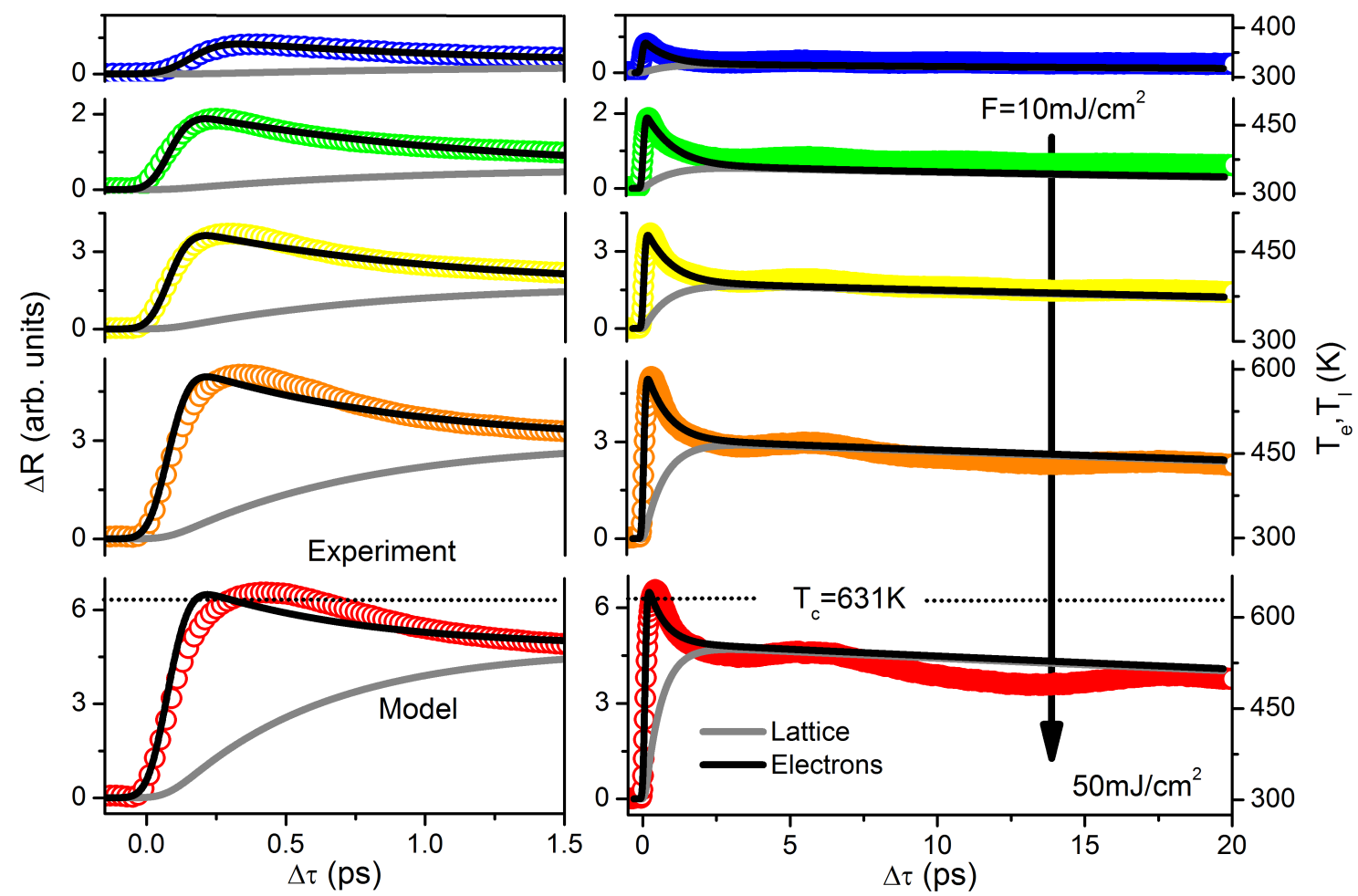

Figure 1.14: Reflectivity data (circles) and the extracted lattice (gray lines) and electron (black lines) temperatures for the $15 \mathrm{~nm}$ nickel film for pump fluences from $10 \mathrm{~mJ} / \mathrm{cm}^{2}$ to $50 \mathrm{~mJ} / \mathrm{cm}^{2}$. (Data has been published in [6]).

netization data, the fast decay in the reflectivity data of these thin films leads to higher peak temperatures, when using the above described method for temperature calibration. Choosing an earlier time after excitation, $\Delta \tau<20$ ps for temperature calibration can not assure an equilibrium temperature between the spins and the electrons, because at these high fluences the slope of the magnetization relaxation is still rather high. Choosing later times for temperature calibration $(\Delta \tau>20 \mathrm{ps})$, leads to even higher electron peak temperatures, because in this regime the magnetization relaxation already too slow. That means that the temperature determined from the magnetization data at around $\Delta \tau=30 \mathrm{ps}$ or $\Delta \tau=20 \mathrm{ps}$, does not differ significantly from that determined at $\Delta \tau=20 \mathrm{ps}$. However, the steep slope in the reflectivity data calibrated to this temperature would show even higher peak temperatures around $\Delta \tau<0.5 \mathrm{ps}$.

The extraction of the electron temperatures from reflectivity data for the $40 \mathrm{~nm}$ nickel film is a more sophisticated task with more complicated underlying mechanisms than the approximation used for the nickel films up to $20 \mathrm{~nm}$. The data in appendix $\mathrm{B}$ show a sign change for $\Delta R$ in the elevated equilibrium state, making the extraction of $T_{e}$ using the above described procedure impossible.

The analysis shows that this method is appropriate to determine electron tem- 


\subsection{Experimental Results}

peratures for nickel films with thicknesses around the penetration depth. Figure 1.14 shows the reflectivity data for the $15 \mathrm{~nm}$ nickel film and the extracted electron and lattice temperatures $3^{3}$ used for the simulations of magnetization dynamics in [6]. The experimental data and the curves fitted from the $2 \mathrm{~T}$ model are in excellent agreement and lead to simulated magnetization dynamics curves which match the experimental data (see figure 1.15). For other thicknesses, this method needs further expansion. The overestimation of the peak electron temperatures in the thinnest films might be excluded, by choosing more transparent substrates. Such substrates would not contribute to the reflected signal, leaving pure nickel data for the analysis. For the films thicker than $20 \mathrm{~nm}$, the deviation of electron temperature in the probed region, compared to the $20 \mathrm{~nm}$ thick film can be considered as small, because for those films, the amount of the per layer absorbed power density does not increase significantly with the thickness. Therefore the data recorded for the $20 \mathrm{~nm}$ thick film might be used for further analysis of the thicker nickel films, and will show whether the discrepancies are significant. Further time-resolved reflectivity experiments with nickel film thicknesses between $20 \mathrm{~nm}$ and $40 \mathrm{~nm}$ will show how the reflectivity changes with the thickness and whether the results allow conclusions on the electron temperature for film thicknesses around or even above $40 \mathrm{~nm}$.

\subsubsection{Demagnetization Data and Spin Temperatures}

Figure 1.15 shows an application example of the thermal model. Ultrafast magnetization dynamics simulated by solving the LLB equation and experimental data of a $15 \mathrm{~nm}$ nickel film are plotted simultaneously for comparison. The experimental data are represented by the open circles, the modeled data by the black lines. To keep the conditions for both, the simulation and the experimental data as close as possible, all parameters used in the experiments, given in section 1.3.1 were adopted as boundary conditions in the simulations. The electron temperatures were extracted from reflectivity measurements. This data, published in [6] shows excellent agreement with the experimental data for film thicknesses around the optical penetration depth, $\Lambda_{\mathrm{opt}} \approx 15 \mathrm{~nm}$. This shows that the LLB equation is capable of predicting magnetization dynamics, even at electron temperatures above $T_{C}$ accurately. Further, this is an indication that the electron temperatures extracted from the reflectivity data are in acceptable agreement to the actual temperatures. This procedure is adequate for films thicker than the penetration depth of the laser light, up to around $20 \mathrm{~nm}$.

\footnotetext{
${ }^{3}$ Note that the extracted electron temperatures are lower than those presented in appendix $B$ That is, because originally the simulations were performed for a classical spin and the temperature was determined using the Langevin function. In future the simulations for a quantum mechanical spin $S=1 / 2$ will be possible, therefore the extracted temperatures presented in appendix $\mathrm{B}$ are determined via the Brillouin function (see section 1.3.3).
} 

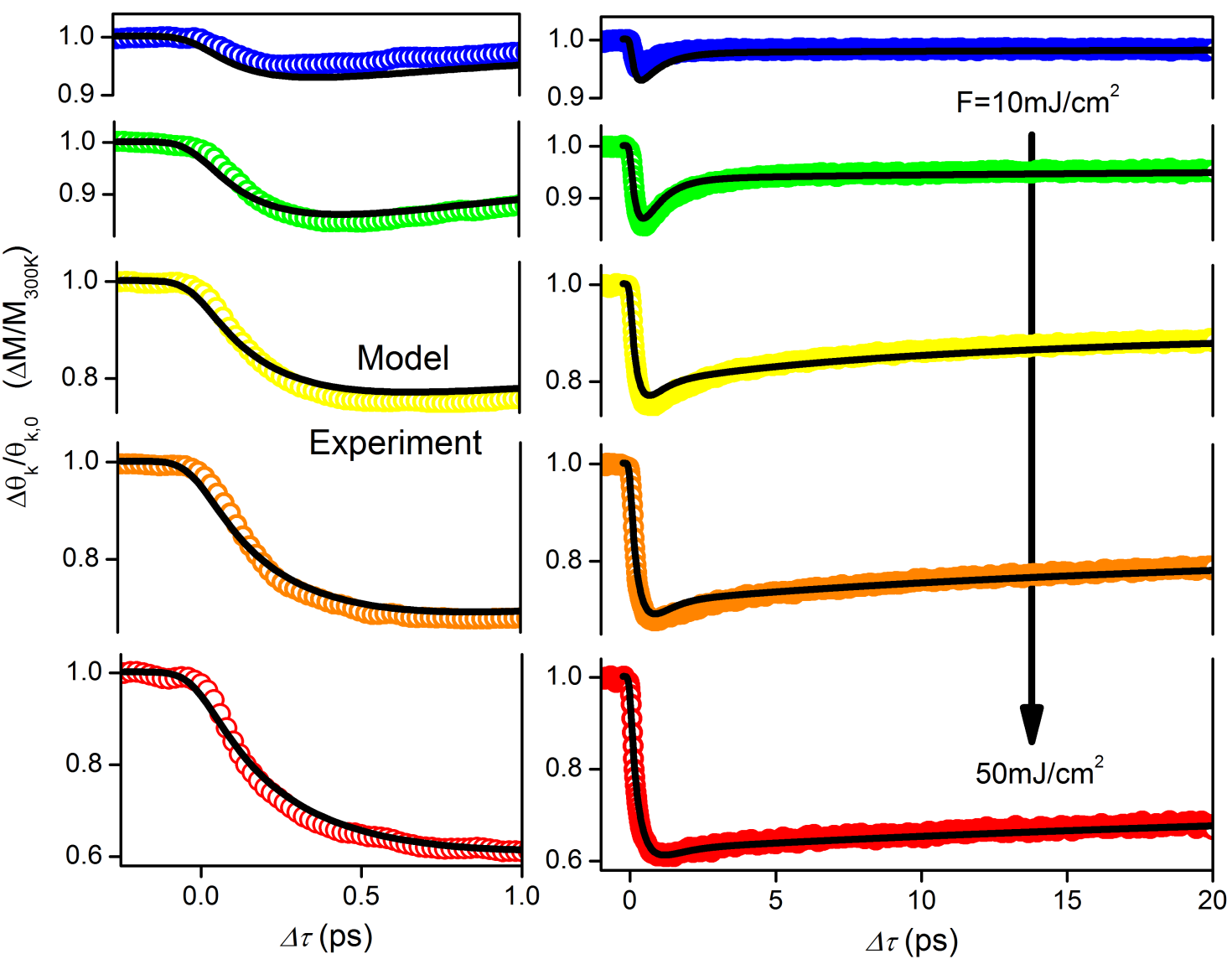

Figure 1.15: Experimental data (colored circles), and thermal model LLB simulations (black lines). The data shows magnetization dynamics on the $15 \mathrm{~nm}$ film (Data has been published in [6]).

For a quantitative analysis, the analytic solution of the 3T model formula [18]

$$
\begin{aligned}
\frac{\Delta M(t)}{M_{300 \mathrm{~K}}}=\left\{\left[A_{1} F\left(\tau_{0}, t\right)-\right.\right. & \frac{\left(A_{2} \tau_{E}-A_{1} \tau_{M}\right)}{\tau_{E}-\tau_{M}} e^{-t / \tau_{M}}- \\
& \left.\left.\frac{\tau_{E}\left(A_{1}-A_{2}\right)}{\tau_{E}-\tau_{M}} e^{-t / \tau_{E}}-A_{3} e^{-t / \tau_{M, r e}}\right] \Theta(t)\right\} * G(t)
\end{aligned}
$$

was fitted to both, the LLB modeled and experimental magnetization dynamics spectra. The first term describes the arrival of the ultra short laser pulse and the amount of the deposited energy. The second term describes the sudden demagnetization, and determines the demagnetization time $\tau_{M}$. The third term describes the relaxation, and determines the relaxation time $\tau_{E}$. The fourth term is an extension, introduced in [6], adding a second relaxation time $\tau_{M, r e}$. This second relaxation time is necessary for magnetization relaxation at high pump fluences. The whole function is multiplied by a step function $\Theta(t)$, representing the sudden drop of magnetization, and convoluted by $G(t)$, representing the temporal expansion of the laser pulse. 


\subsection{Experimental Results}

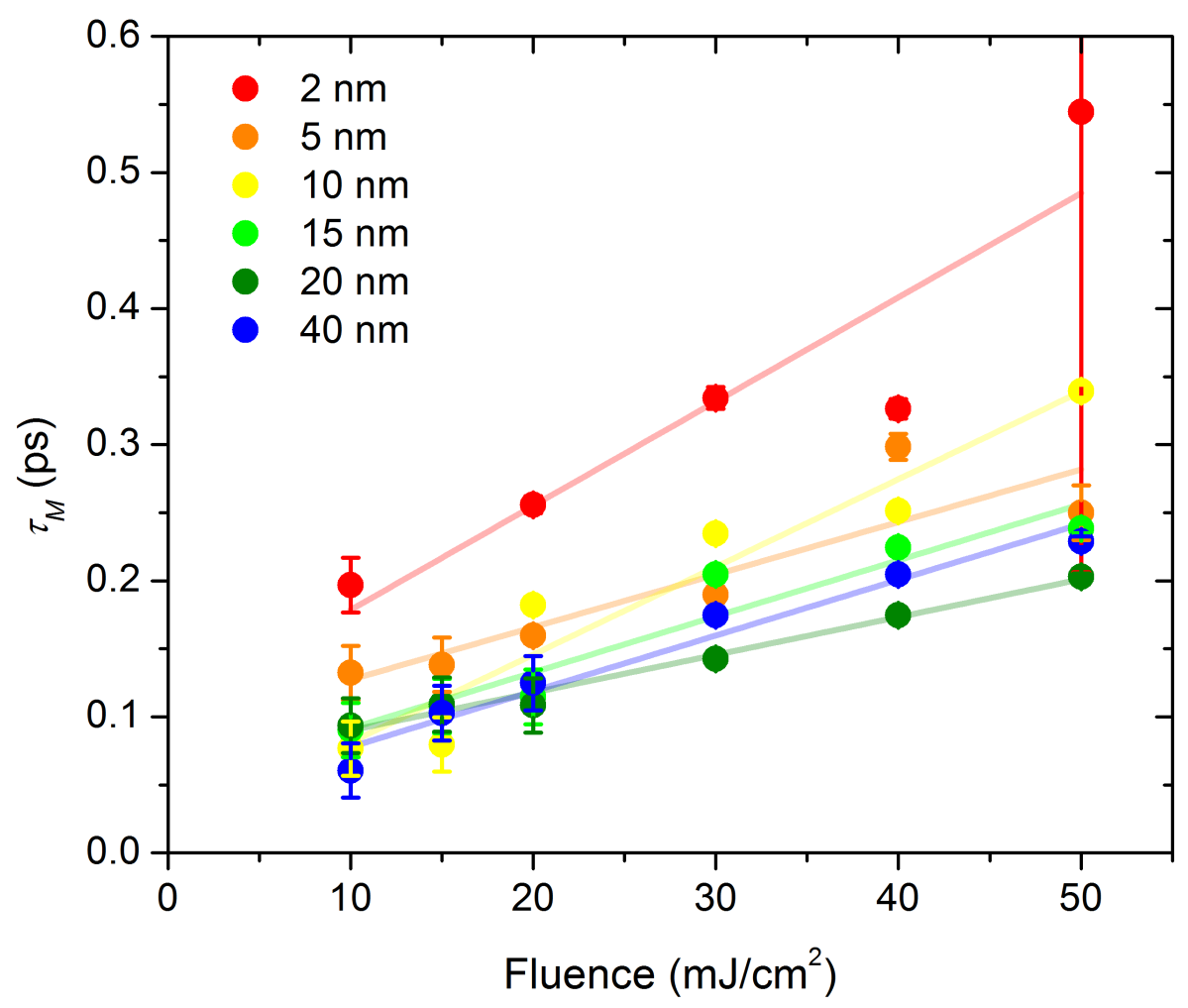

Figure 1.16: Demagnetization times $\tau_{M}$ extracted by fitting equation 1.20 to the experimental data.

The implementation of a second relaxation time can be concluded, from the data shown in figure 1.5. There it is shown that at low excitation temperatures of the electron system, the magnetization relaxes within $\sim 2 \mathrm{ps}$ after excitation into a quenched magnetization equilibrium state and remains there for at least the next 15 ps. Whereas, at higher temperatures and higher maximum demagnetization, the first relaxation represented by a dip in the data, decreases in amplitude, leaving the system in a higher demagnetization state. From that state, the magnetization slowly relaxes into the quenched equilibrium, expressed in equation 1.20 by including $\tau_{M, r e}$.

The modeling of ultrafast magnetization dynamics applying the LLB equation becomes more problematic for film thicknesses differing from the optical penetration depth. For the $40 \mathrm{~nm}$ thick nickel film, the simulations have not been performed, because the determination of the electron temperature has not been possible (see discussion in the previous chapter).

In the case of films thinner than the optical penetration depth, as already discussed in the previous section, the reflectivity data for the thin nickel films does not lead to comparable magnetization curves obtained from LLB simulations and experiments, because the electron temperatures determined from the reflectivity data are overestimated. Measurements on films deposited on more transparent substrates than $\mathrm{Si}$, e.g. $\mathrm{MgO}$ might lead to more reliable results. 


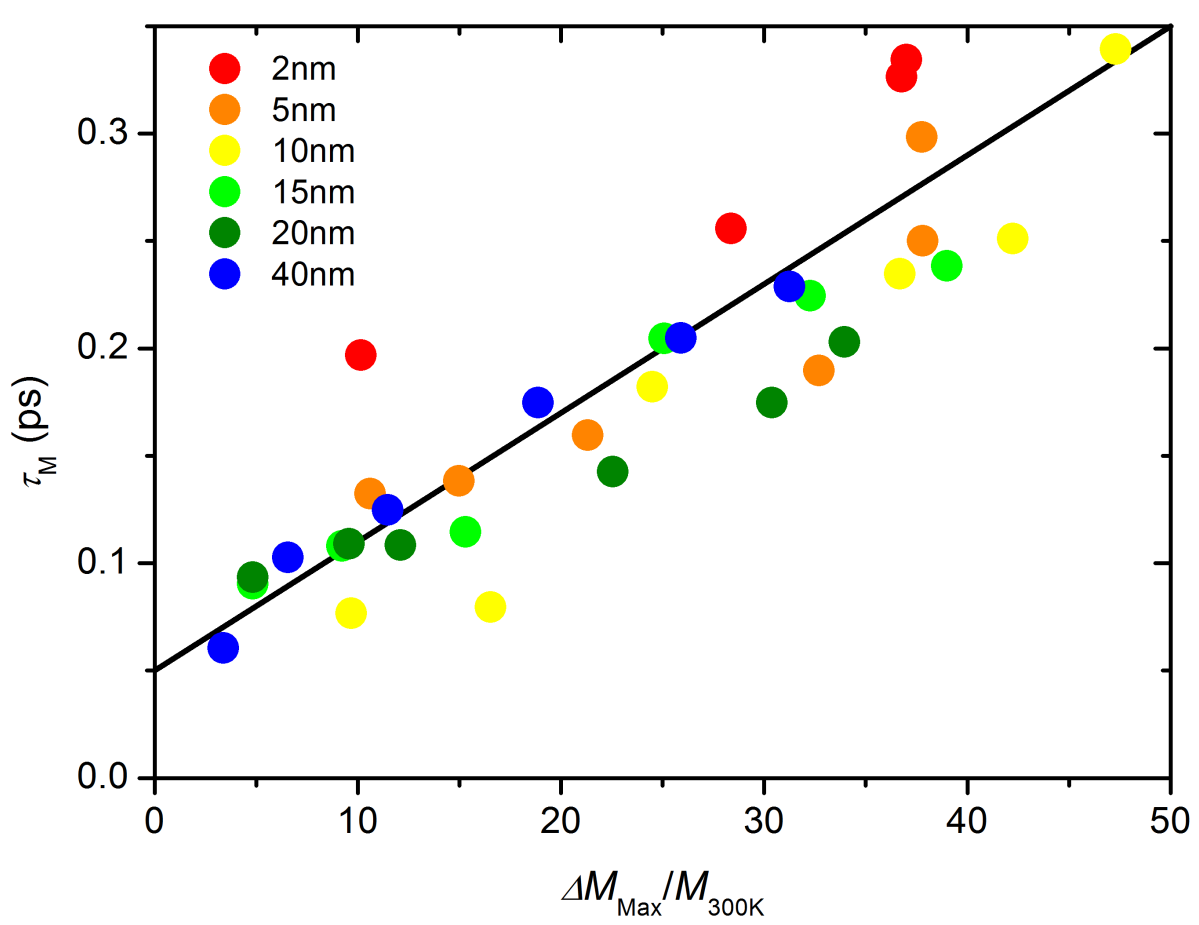

Figure 1.17: Demagnetization times $\tau_{M}$ plotted against the maximum demagnetization $\Delta M_{\mathrm{Max}} / M_{300 \mathrm{~K}}$. The black line is a guide to the eye.

At this point, the excitation and relaxation processes for all examined nickel films and all applied pump fluences will be discussed on the basis of the above given equation 1.20 . The times extracted by fitting this formula to the experimental data show a qualitative agreement with the predictions made by the thermal model. The experimental data and the fits are attached in appendix B.

The extracted demagnetization times $\tau_{M}$ are plotted against the pump fluence in figure 1.16 for all examined nickel films. In the vicinity of the Curie temperature, the dynamics in the LLB equation predicts a slowing down of the spin-flip processes, due to second order transition of magnetization, displayed in the longitudinal relaxation and susceptibility. This suggests in general a slower demagnetization and larger $\tau_{M}$ with increasing pump fluence, especially in the data, where the electron temperature reaches or even exceeds $T_{C}$. According to the calculations in section 1.4 .2 , displayed in figure 1.10, the absorbed power density is the highest for the thinnest nickel films, and decreases with increasing film thickness, for every pump fluence.

For the thicker samples $\left(d \geq \Lambda_{\text {opt }}\right)$, the absorbed power density ceases to increase with the thickness at any applied fluence. That means, $\tau_{M}$ is expected to increase stronger for the thinner films with increasing pump fluence. For the thicker films, $\tau_{E}$ should not differ much between films, at any given fluence. The temperatures extracted from the $2 \mathrm{~T}$ and 3T model, suggest maximum electron temperatures above $T_{C}$, especially for the thinner films. At the same time, the maximum spin temperatures are $\lesssim 0.9 T_{C}$ at the highest fluence, for all examined nickel films. Besides that, 


\subsection{Experimental Results}

for the thinner films, maximum electron temperatures are reached at a medium fluence, and decrease again with further increasing fluence, whereas the maximum spin temperatures increase continuously with the pump fluence. This discrepancy might cause some deviation from the assumed trends.

One clear trend visible in figure 1.16 is the increasing of $\tau_{M}$ for each film with the pump fluence. This mirrors the increasing contribution of the parallel relaxation when approaching $T_{C}$. Also the demagnetization times of the three thinnest films tend to be higher than those of the three thickest. Further, the three thickest films show approximately the same demagnetization times for each fluence with similar slopes for $\tau_{M}$ against the pump fluence, increasing by a factor of two from the lowest to the highest applied pump fluence. The three thinnest films each show a different slope and the slopes are in general steeper than those for the thicker films. These characteristics show a consistency with the model predictions, for the extracted temperatures. At low fluences, the $10 \mathrm{~nm}$ nickel film does not show a larger demagnetization time than the thicker films. Although the maximum spin temperatures reach as high values, as those of the $2 \mathrm{~nm}$ and the $5 \mathrm{~nm}$ films, the electron temperatures at these pump fluences are lower and do not even reach $T_{C}$. The electron bath does not provide high temperatures for a long enough time period sufficient for transition processes, caused by the contribution of the longitudinal susceptibility $\chi_{\|}$and spin-flip rate $\Gamma_{\|}$, and a slowing down of the demagnetization can not be initiated. The $5 \mathrm{~nm}$ film shows a relatively small $\tau_{M}$ at the high pump fluences. This can be ascribed to an artifact arising from the fitting procedure, and will be discussed in a separate section 1.4.5. For clarity, figure 1.17 shows the demagnetization time $\tau_{M}$ plotted against the maximum demagnetization $\Delta M_{\mathrm{Max}} / M_{300 \mathrm{~K}}$. The data follows in general a clear trend, $\tau_{M}$ increasing with the maximum demagnetization, and thus with the maximum temperature. This depiction is an additional indication that the critical slowing down occurs, and starts to show its significance, already at low temperatures $\sim 0.5 T_{C}$, as calculated in [5]. The data points follow the black line, which is a guide to the eye.

The extracted relaxation times are plotted in figure 1.18 and 1.19 . While the first relaxation time $\tau_{E}$ in figure 1.18 does not exhibit any significant change, slightly increasing from $1 \mathrm{ps}$ to $3 \mathrm{ps}$ with the pump fluence, for the thicker nickel films, it does not show any clear fluence-dependent trend for the both thinnest nickel films. Compared to simulations for electron temperatures as high, as were extracted from the reflectivity data for the thinnest films [5], the maximum demagnetization and thus the maximum spin temperatures in the experimental data for these films are rather low. However, at high fluences the experimental magnetization dynamics data of these films exhibit features theoretically predicted for magnetization dynamics at higher or even total demagnetization, namely the much slower second relaxation. The exhibition of a dominant, i.e. a slow second relaxation process and a high $\tau_{M, r e}$, shows the limits of this fitting procedure. Then the first relaxation time $\tau_{E}$ cannot be reliably determined. This shows in the decreasing $\tau_{E}$ at high fluences for the three thinnest films, especially for the $2 \mathrm{~nm}$ film and the $10 \mathrm{~nm}$ film. Nevertheless, 


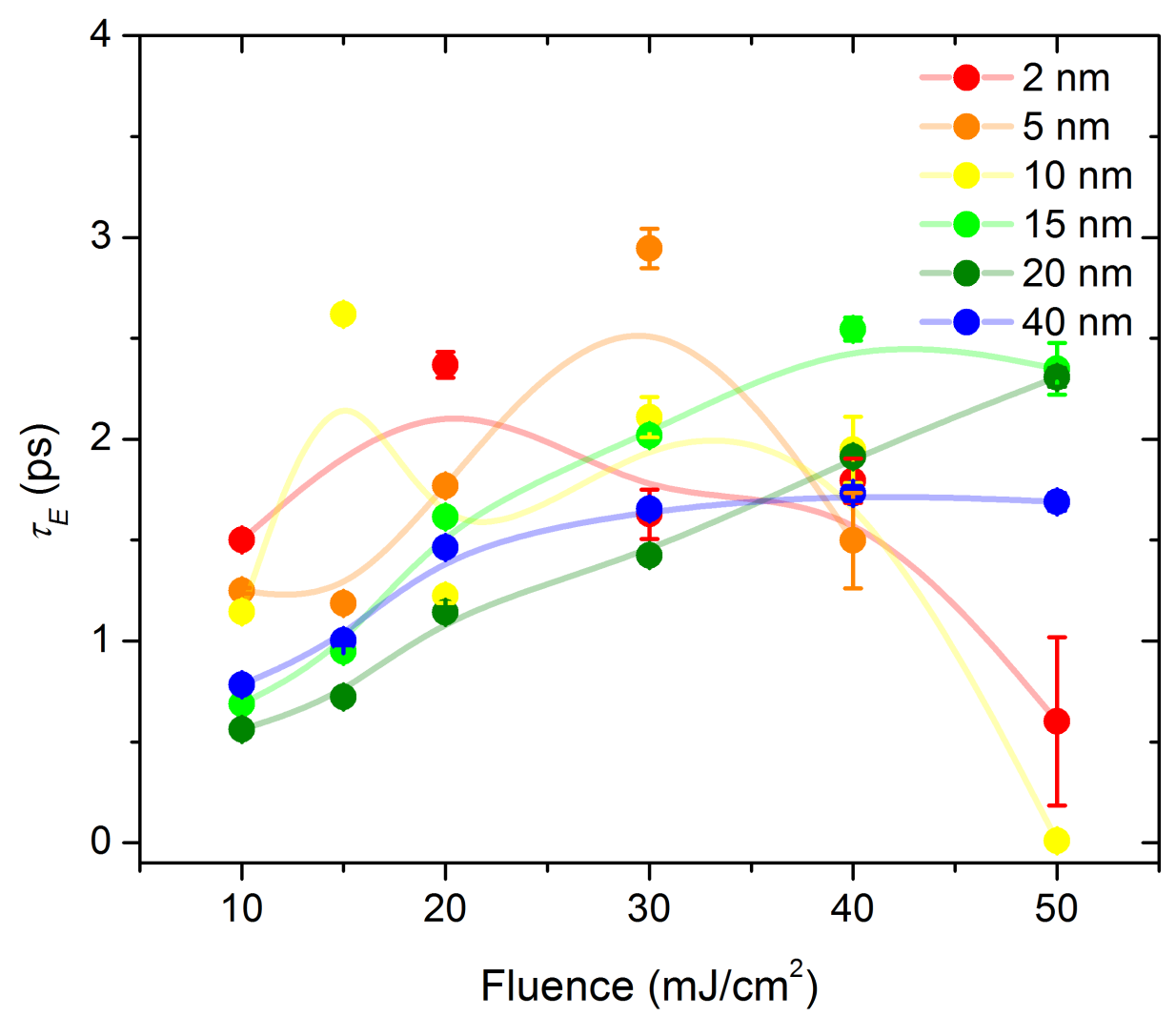

Figure 1.18: Relaxation times $\tau_{E}$, extracted from the fits to the magnetization dynamics experimental data. The lines are a guide to the eye, outline the progress of $\tau_{E}$ with the pump fluence.

the extracted values remain largely in the same range as those of the thicker films, between $0.5 \mathrm{ps}-3 \mathrm{ps}$. That means, they are in general in agreement with the model.

At fluences, starting with $20 \mathrm{~mJ} / \mathrm{cm}^{2}$ for the thinner films and with $30 \mathrm{~mJ} / \mathrm{cm}^{2}$ for the thicker films, larger excitations out of equilibrium, lead to a delayed time at which the spin system reaches the equilibrium at an elevated temperature. This is the slowing down, mirrored in the second relaxation time $\tau_{M, r e}$. The decreasing amplitude of the first relaxation and the second relaxation slowing down with increasing electron temperature, are the result of correlation loss at high temperatures due to vanishing micromagnetic exchange [5]. At maximum demagnetization, where the electron temperatures are high, the longitudinal relaxation rate $\Gamma_{\|}$decreases, increasing the longitudinal relaxation time $\tau_{\|}$. That means, first, the higher the demagnetization due to correlation loss and low relaxation rates, the lower the first relaxation amplitude, leading to a decreasing dip with increasing temperature in the data shown in figure 1.5. Second, the electron temperature relaxes into lower temperature regions within $\sim 0.5 \mathrm{ps}$, which is much faster than the extracted values for $\tau_{E} \approx 1 \mathrm{ps}-3 \mathrm{ps}$, leaving the magnetization in a quenched state, close to maximum demagnetization. The longitudinal relaxation slows down and looses significance, leaving the much lower perpendicular relaxation rate. That means, the higher the 


\subsection{Experimental Results}

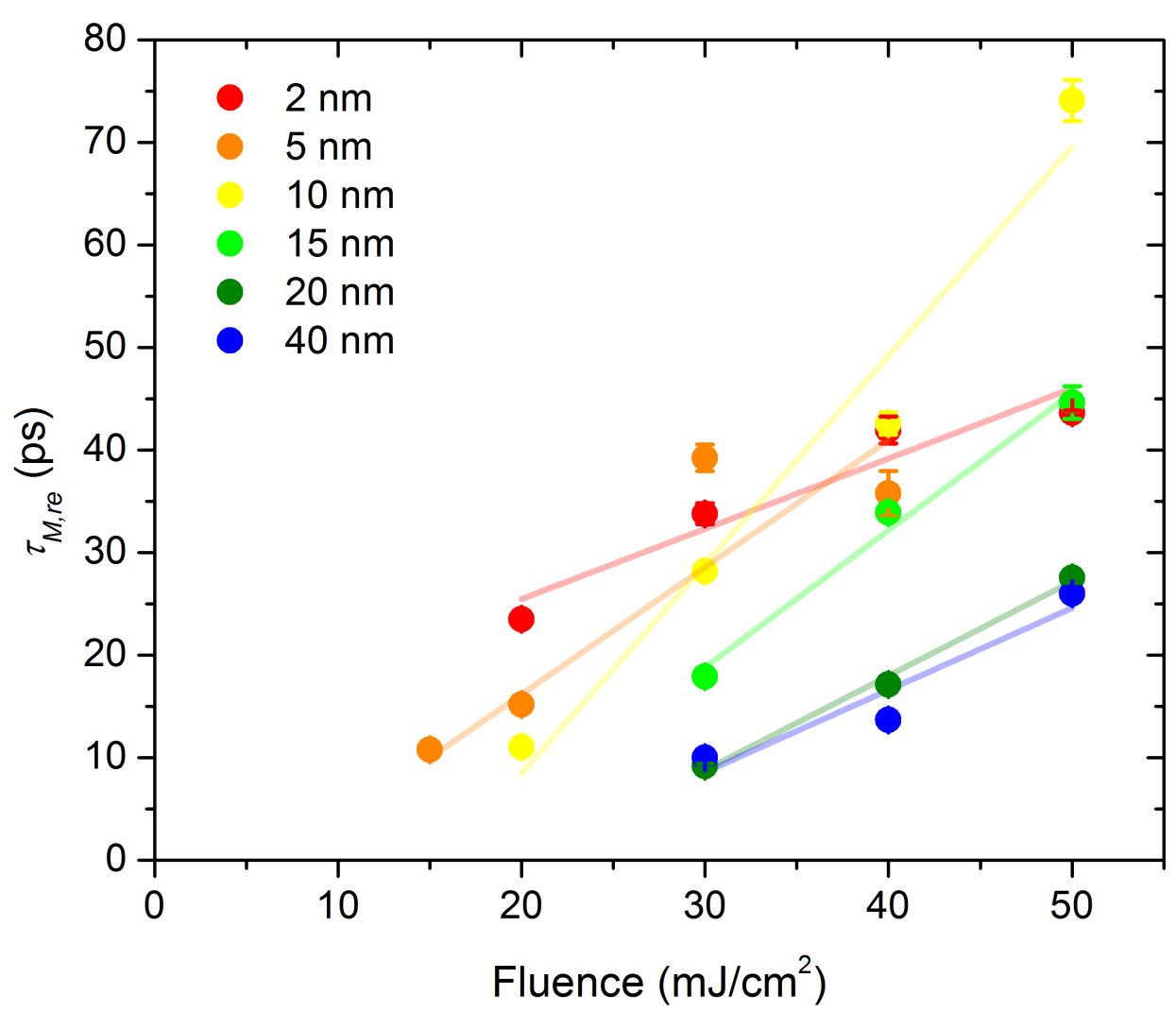

Figure 1.19: Relaxation times $\tau_{M, r e}$, extracted from the fits to the experimental data. The lines are a guide to the eye, pointing out the tendency of $\tau_{M, r e}$ increasing with the pump fluence for each nickel film.

demagnetization, the slower the relaxation, because coming from a higher disorder the restoration of the magnetization takes more time.

The experimental data exhibits this behavior, specifically the thinnest nickel films show a large $\tau_{M, r e}$, although a total demagnetization is not reached even at high fluences. The decreasing amplitude of the first relaxation (represented by $\tau_{E}$ ) with increasing pump fluence, $F>30 \mathrm{~mJ} / \mathrm{cm}^{2}$ can be identified throughout the experimental data for all nickel film thicknesses shown in appendix B. The relaxation times of the second relaxation process, $\tau_{M, r e}$ plotted in figure 1.19 increase with the pump fluence for each nickel film, indicated by the straight lines, which are a guide to the eye. The higher relaxation times for the thinner films are consistent with their higher demagnetization times, and the higher electron temperatures. However, the extracted high electron temperatures suggest a higher maximum demagnetization, for the thinner films. Even though the electron temperatures are overestimated (see discussion above), they do not explain the comparatively small maximum demagnetization in the thinner films. Because of the large relaxation times one expects a large demagnetization. In the experimental data, the second relaxation takes place on a time scale from $10 \mathrm{ps}-50 \mathrm{ps}$ (see figure 1.19 . The $10 \mathrm{~nm}$ nickel film shows a 
slight deviation from the trend in the second relaxation time $\tau_{M, r e}$. At $20 \mathrm{~mJ} / \mathrm{cm}^{2}$ pump fluence, $\tau_{M, r e}$ is rather small. This behavior can be explained by the relatively low maximum electron temperature obtained in this measurement. This low temperature leads to a lower elevated temperature, from which the equilibrium state is reached faster, resulting in a small $\tau_{M, r e}$. The high $\tau_{M, r e} \approx 75 \mathrm{ps}$ at $F=50 \mathrm{~mJ} / \mathrm{cm}^{2}$, contains a large error due to the limitations of the fitting procedure. In fact, this relaxation time may be even larger.

In summary, the times extracted using equation 1.20 largely agree with the trends predicted by the thermal model, making the LLB equation a promising candidate for the description of magnetization dynamics in a wide range of materials. The missing link is a reliable access to the electron temperature, as this is the only parameter required for input. The method of electron temperature extraction from reflectivity measurements is possible, even for the film thicknesses $\left(d<\Lambda_{\mathrm{opt}}\right.$ and $\left.\Lambda_{\mathrm{opt}}<d\right)$, but requires a lot of expertise and a thorough evaluation of the data.

\subsubsection{Limitation of the 3T Fitting Procedure}

In most cases the analytical solution of the $3 \mathrm{~T}$ model (equation 1.20 is an adequate approach to analytically determine the relaxation times and is sufficient for a quantitative analysis to either compare data generated in simulations of the LLB equation to experimental data, or to check, whether the experimental data shows the trends predicted by the thermal model. The non-unambiguity of the analysis arises from the fact, that the time range of the examined process has to be divided into three regions, and a $\chi^{2}$ would have to be calculated for each region carefully. The three regions of interest are the demagnetization, the region around the maximum demagnetization and the relaxation. They overlap and change for each data set, varying for each fluence and each sample, making an objective analysis difficult. The limits of the model are reached at high pump fluences. The analytic solution to the three temperature model is derived in the low perturbation limit, and by neglecting the spin specific heat, which is orders of magnitude smaller than the electron specific heat. Besides that, an instantaneous rise of the electron temperature, with photon absorption of the laser pulse is assumed [18]. The extension by the third relaxation time $\tau_{M, r e}$ provides the ability to describe the dynamics on a wider temperature range.

The challenges will be discussed based on the data in figure 1.20. The left graph shows experimental data of all examined nickel films perturbed by the highest pump fluence $\left(50 \mathrm{~mJ} / \mathrm{cm}^{2}\right)$. Although less pronounced than the dips in the model simulations [5] at this fluence, the characteristics predicted by the thermal model show up distinctly in the data to the three thickest films. In particular the amplitude of the first relaxation after maximum demagnetization is distinguishable, stating clearly, where the second relaxation begins. The first relaxation amplitude decreases with increasing demagnetization from the $40 \mathrm{~nm}$ to the $15 \mathrm{~nm}$ film, as expected from the model. For these data, the demagnetization and relaxation times extracted by the 

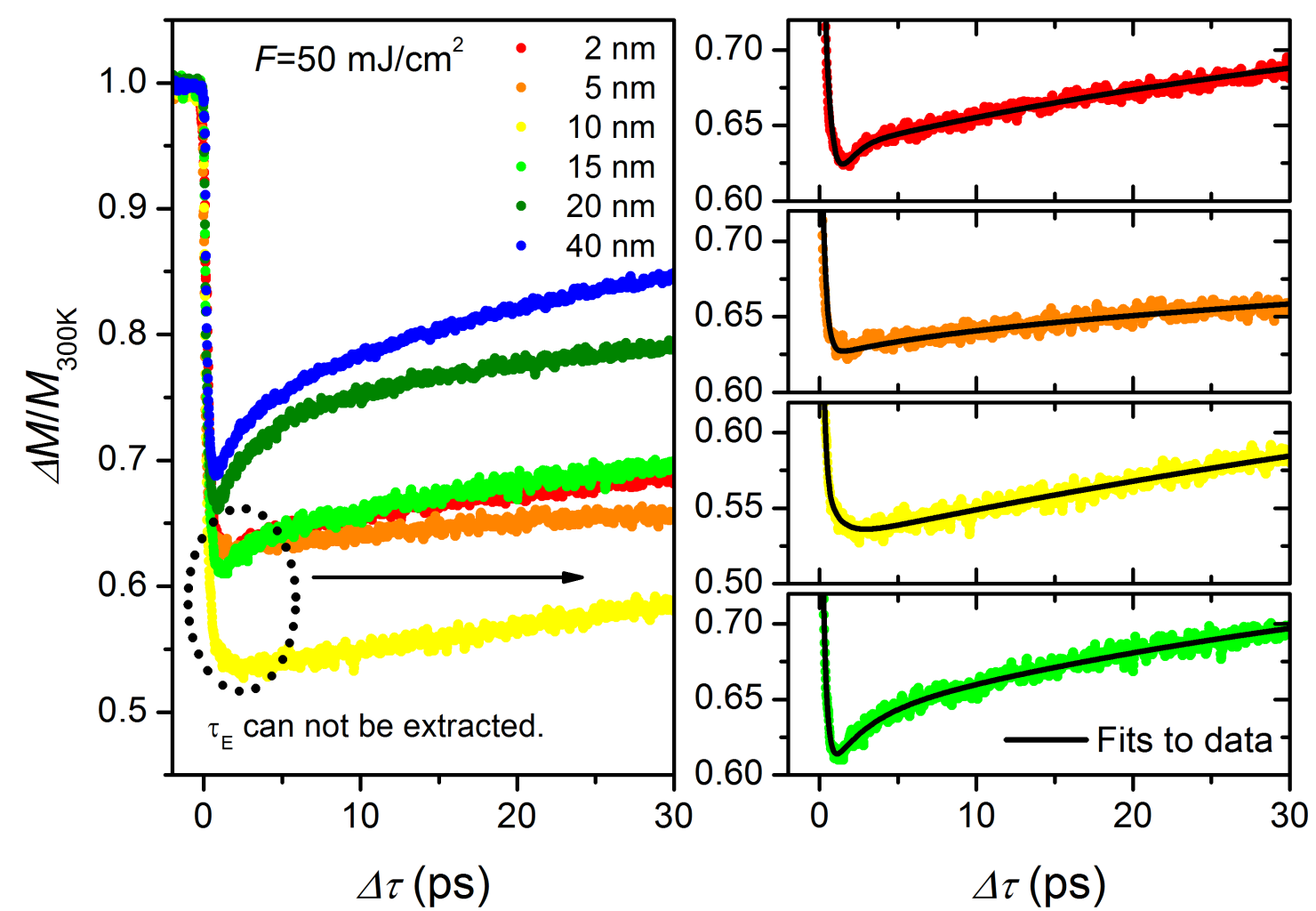

Figure 1.20: Magnetization dynamics, all nickel films at the fluence $50 \mathrm{~mJ} / \mathrm{cm}^{2}$ (left). Enlarged, (right) the same data, magnified around the point of maximum demagnetization and the relaxation process for the nickel films $2 \mathrm{~nm}-15 \mathrm{~nm}$.

fit of equation 1.20 show all consistent with model predictions without larger deviations. The evaluation becomes more complicated for the data from the thinner samples. These circumstances are highlighted in figure 1.20 right, magnified around the point of maximum demagnetization. Especially in the spectra of the $10 \mathrm{~nm}$ and $5 \mathrm{~nm}$ films the first relaxation is not even detectable. Therefore, both extracted relaxation times require a cautious consideration here. At first view, the $2 \mathrm{~nm}$ data exhibits a 'dip', which might possibly be a feature of the first relaxation process, but it could also be ascribed to noise. The characteristic of the first relaxation is important to be present and distinguishable in the data, otherwise, either the demagnetization or the second relaxation times will be attributed larger values than they actually require. Further, the total reduction of the first relaxation process amplitude is predicted in the simulations for much higher demagnetizations than were obtained in the experiments. This supports the statement from the previous section that the data for the thinner samples exhibits features predicted for higher demagnetizations than were actually obtained in the experiments.

The difficulties, working with equation 1.20 show that the obtained relaxation time values need a thorough consideration, before conclusions can be drawn. Whenever possible, $\tau_{E}$ can be extracted from reflectivity data. Because the specific heat of the spin system $C_{S}$ is much smaller than the specific heat of the electron system and 
the lattice, $\tau_{E}$ in the solution of the $2 \mathrm{~T}$ model and the $3 \mathrm{~T}$ model describe the same relaxation [45]. The benefit from extracting $\tau_{E}$ from reflectivity data is reducing the degrees of freedom in the fit formula, equation 1.20 . However, the nickel reflectivity data at high pump fluences does not exhibit a pronounced $\tau_{E}$. Additionally, the oscillations of the stress waves showing in the data at high fluences do not lead to the extraction of reliable values for $\tau_{E}$ from reflectivity data.

\subsubsection{More Approaches to Magnetization Dynamics Beyond $T_{C}$}

There are two other recently suggested approaches describing the ultrafast spin dynamics near and beyond the Curie temperature $T_{C}$. One, also based on a micromagnetic model for the spin disorder (m3TM), averaging electronic properties and the other based on electronic effects, averaging magnetic degrees of freedom. Both models have been compared to experimental data and are capable of reproducing those. Therefore, they have to be included in the current discussion.

\section{The Micro-magnetic Three Temperature Model m3TM}

This model, first presented in [38], assumes, as well as the thermal model, ElliottYafet type (electron-phonon) spin-flip scattering processes on the microscopic scale as the dominant underlaying microscopic mechanism. Other than that, the model is based on the three temperature model, ascribing temperatures, heat capacities and coupling constants to the three baths, the electrons, the spins and the lattice. The result are three coupled differential equations describing the change of temperature after ultra short heating. The equations describing the temporal temperature change of the electron and lattice system are the same as those given in section 1.3.3. The equation describing the magnetization dynamics was derived based on a simplified model Hamiltonian for spinless free electrons and phonon oscillations based on the Einstein model. Spin excitations were treated via the mean-field Weiss model. Similar to the thermal model, the spin specific heat is considered much smaller than the electrons $C_{s} \ll C_{e}$, assuming an instantaneous thermalization of the spin bath with the electron bath. The electron specific heat increases linearly with temperature, and the lattice specific heat is kept constant. The resulting temperature dependent magnetization is:

$$
\frac{d m}{d t}=R m \frac{T_{l}}{T_{C}}\left(1-m \operatorname{coth}\left(\frac{m T_{C}}{T_{e}}\right)\right) .
$$

Here $m=M / M_{S}$, the factor $R$ has the dimension $(1 / \mathrm{s})$ and is a material specific scaling for the demagnetization rate. It is expressed by the relevant magnetic parameters given by $R \propto a_{s f} T_{C}^{2} / \mu_{a t}$. The Elliott-Yafet type scattering is described by the probability $a_{s f}$ that an electron flips its spin on emission or absorption of a phonon. The parameter $\mu_{a t}$ is the atomic magnetic moment. $R$ determines two types of materials. Materials exhibiting a large $R$ on one hand are expected to show type I magnetization dynamics. There the demagnetization is completed before the 


\subsection{Experimental Results}

electron-phonon equilibration is achieved. In general that means that the same features are expected for type I dynamics, as in the dynamics described by the thermal model. First, a fast demagnetization and a slower recovery at low fluences and a critical slowing down at higher fluences. Determining $a_{s f}$ at different pump fluences reveals that it stays constant with changing pump fluence. That means, determining $a_{s f}$ for a material can predict the dynamics triggered by any pump fluence. The constancy of $a_{s f}$ for varying pump fluences and thus different temperatures together with the critical slowing down of demagnetization shows that non-thermal electrons are not important for the demagnetization, because then the demagnetization would proceed faster at higher fluences.

On the other hand, materials with a small $R$ are expected to show type II dynamics. These dynamics exhibit a fast demagnetization followed by a second slower demagnetization. The first demagnetization is not efficient enough to establish a thermal equilibrium of the spin system during electron-phonon equilibration. The efficiency increases at higher temperatures, leading to a second demagnetization. Type II dynamics can also be deducted from the thermal model, using the LLB equation. The equivalence of the dynamics modeled using the LLB equation and the here introduced m3TM [38] has been shown only recently in [3]. Unlike in the classical treatment of spins $(S \rightarrow \infty)$, for the quantum mechanical spins $(S=1 / 2)$, $\lambda$, the coupling of the spins to the electron bath, is not constant anymore, but can be identified as a function of $R$. Therefore, the LLB equation can simulate magnetization dynamics of materials exhibiting type I and type II behavior, as the coupling of the spins to the bath changes with temperature. Further, there is a possibility to derive a temperature independent coupling to the bath parameter, $\lambda=\lambda_{0}\left(T_{p} / T_{e}\right) .1$

An analysis of equation 1.21 showed [38] that a high $R$ can be achieved in materials with a high spin-flip probability $a_{s f}$, or a high ratio $T_{C} / \mu_{a t}$. For materials with the same $a_{s f}$, the ratio $T_{C} / \mu_{a t}$ was chosen the figure of merit. A small figure of merit indicates a fast demagnetization, and vice versa. This was investigated on $\mathrm{Ni}$ and Co. Both materials show a similar spin-flip probability, determined from simulation of magnetization dynamics using equation 1.21 . However, $T_{C} / \mu_{a t}(N i) \approx 1000 \mathrm{~K} / \mu_{\mathrm{B}}$, and $T_{C} / \mu_{a t}(C o) \approx 800 \mathrm{~K} / \mu_{\mathrm{B}}$. Also the demagnetization times determined for Ni are faster than for Co. A further analysis of the m3TM model published in [40] supports the trends derived from equation 1.21 , by comparing simulations with experiments carried out in the limit of $T \rightarrow T_{C}$.

The m3TM can specifically be compared in the present work based only on the $15 \mathrm{~nm}$ nickel film data, since in [38] among others, also a polycrystalline $15 \mathrm{~nm}$ nickel film was examined. The magnetization in the authors' experiments was quenched by $50 \%$, which leaves only the dynamics using the highest pump fluence, $50 \mathrm{~mJ} / \mathrm{cm}^{2}$ for comparison, where a maximum demagnetization of $40 \%$ was reached. The m3TM model predicts a $\tau_{M}^{\mathrm{m} 3 \mathrm{TM}}=0.21 \mathrm{ps}$, compared to an experimental value of $\tau_{M}^{\exp }=$ $0.16 \mathrm{ps}$. The demagnetization time determined via the LLB equation is $\tau_{M}^{L L B}=$ $0.25 \mathrm{ps}$, for a slightly lower demagnetization, compared to a $\tau_{M}^{\exp }=0.19 \mathrm{ps}$, both determined by fitting the magnetization dynamics curves using equation 1.20 . Using the same procedure to determine $\tau_{M}$ as in [38], the experimental data in the present 

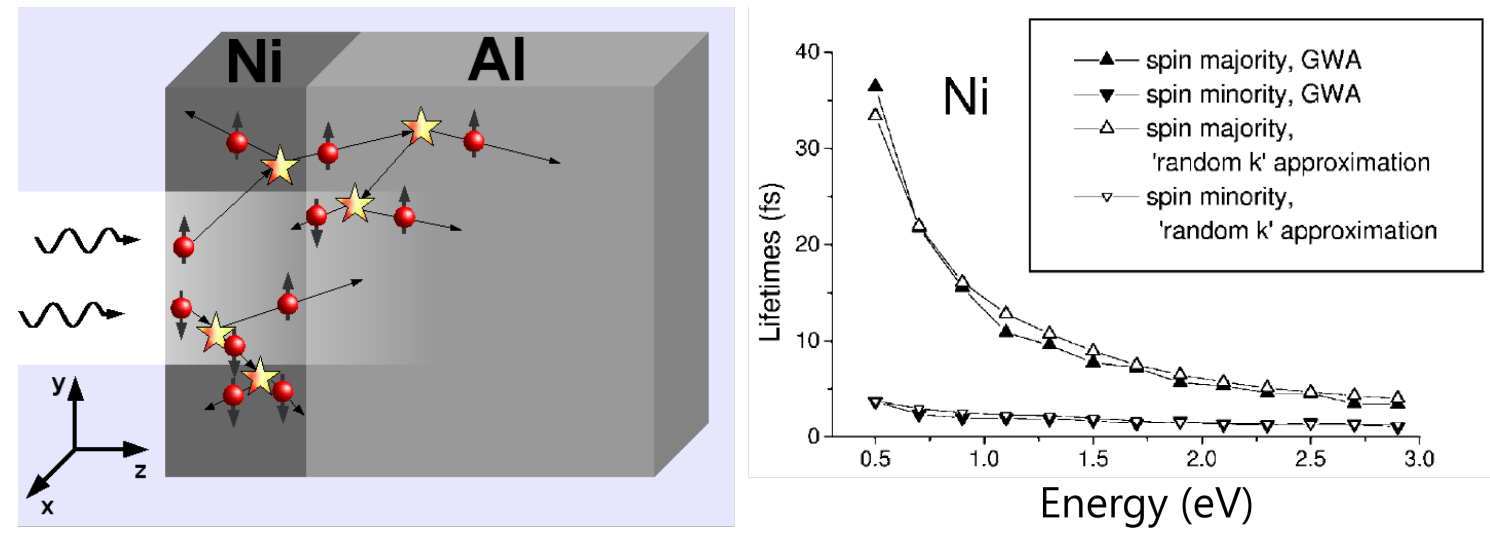

Figure 1.21: Sketch of the spin supper-diffusion mechanism, after laser excitation, the diffusion of electrons is spin dependent (left), taken from [8]. This spin dependent transport is a consequence of spin dependent lifetimes, after excitation above the Fermi level in nickel, at different energies below $2 \mathrm{eV}$ (right, taken from [70].

analysis shows a slightly smaller demagnetization time, $\tau_{M}=0.17 \mathrm{ps}$. The agreement of the extracted demagnetization times using these different models supports the analysis in [3], showing the equivalence of both models.

\section{Spin Super-Diffusion Contributing to Ultrafast Demagnetization}

This second model under discussion is based on purely electronic effects. Unlike the previously introduced models it is not derived from the averaging of electronic properties, but rather from averaging magnetic degrees of freedom.

In [70] (see figure 1.21 (right)) it was shown that electrons above the Fermi level, have different spin-dependent lifetimes in the excited state. Considering photon energies of $1.55 \mathrm{eV}$ for the light pulses generated by Ti:Sapphire lasers (wavelength around $800 \mathrm{~nm}$ ), the lifetimes for majority spin electrons are about 10 times higher than for minority spin electrons in nickel.

On this basis, Battiato et al. developed a model, in which the mechanism for ultrafast demagnetization is the super-diffusive spin transport [8]. In this model a d-band electron is excited by absorbing a photon into the sp-like band. The d-bands are considered localized and thus the electrons in the sp-bands are having a greater mobility. For the mobility of electrons, trajectories were developed, assuming that electrons absorbing photons will be excited into the sp-like bands and start moving in arbitrary directions. During this motion scattering events with other electrons or phonons can lead to arbitrary changes of direction. The developed transport process is inbetween a ballistic and a diffusive process called super-diffusion, described by:

$$
\frac{\partial n(\sigma, E, z, t)}{\partial t}+\frac{n(\sigma, E, z, t)}{\tau(\sigma, E, z)}=\left(-\frac{\partial}{\partial z} \hat{\Phi}+\hat{I}\right) S^{e f f}(\sigma, E, z, t)
$$




\subsection{Experimental Results}

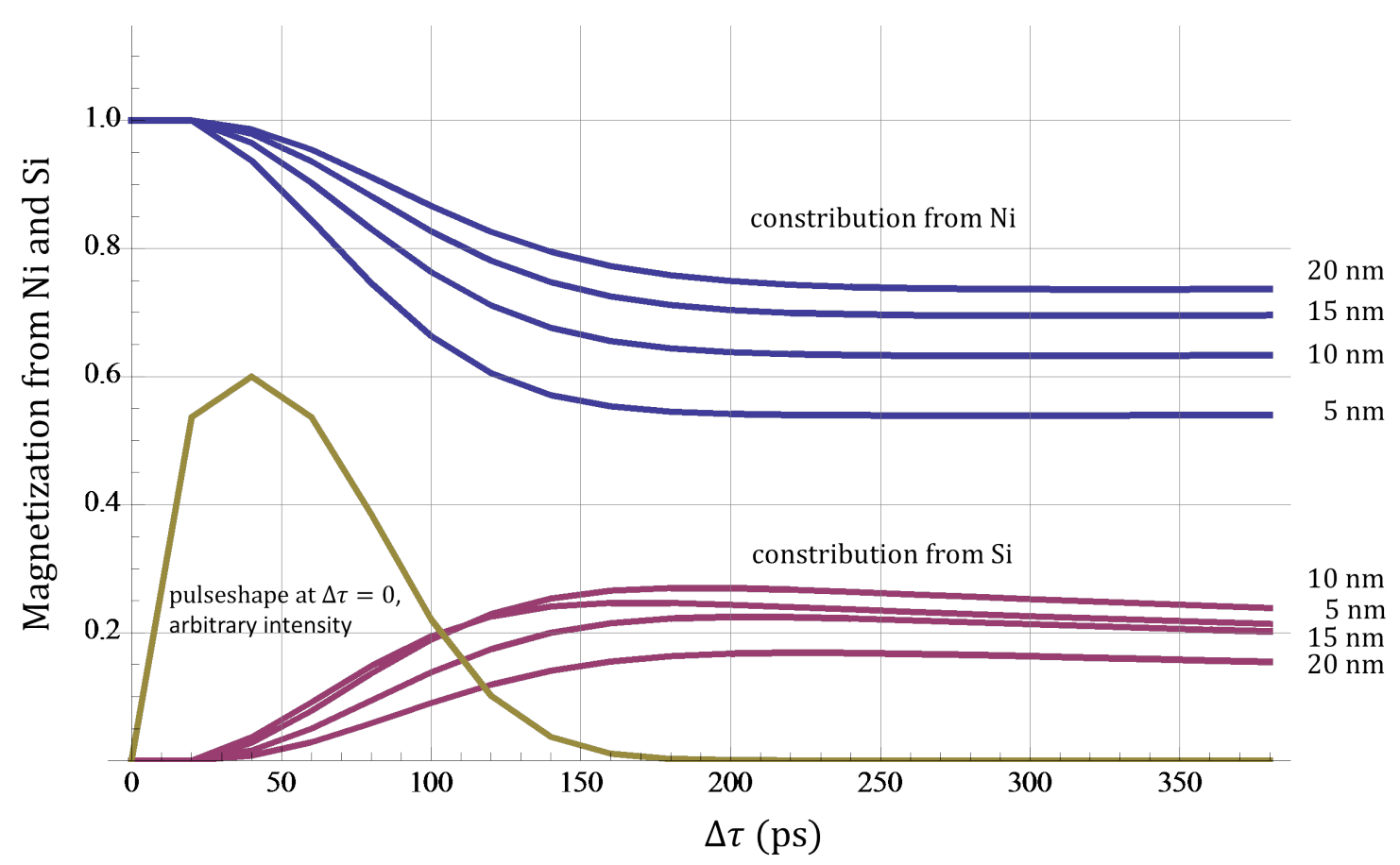

Figure 1.22: Simulation of the demagnetization of Ni films from $5 \mathrm{~nm}$ to $20 \mathrm{~nm}$ using super-diffusive transport properties, and the magnetization of the Si substrate, resulting from the majority spin electrons diffusing from the nickel film. Taken from [9].

where $n$ is the number of excited electrons, $\hat{\Phi}$ is the flux of electrons, $\hat{I}$ the identity operator and $S^{e f f}$ the scattering term. The parameters describing the electrons are $\sigma$ the spin state, $E$ the energy, $z$ the depth of the ferromagnetic film and $t$ the time after excitation, unlike $\tau$ is the lifetime on the excited state. The model was derived such that the spin-dependent density of electrons at the Fermi level across the penetration depth that means, the thickness of the sample, determines the magnetization state.

Several issues lead to the conclusion that this super-diffusive transport process may contribute to demagnetization. First, because majority spin electrons have longer lifetimes, they are more probable to move away than minority spin electrons. Second, the velocities in sp-like bands are $\sim 1 \mathrm{~nm} /$ fs, leading to demagnetization, if they move away from their location. Further, during inelastic scattering with other electrons, a cascade of electrons moving in one direction can be generated.

Applying this model to a $15 \mathrm{~nm}$ nickel film deposited on an $\mathrm{Al}$ substrate and illuminated by an ultrashort laser pulse, leads to the result that due to the diffusion of the electrons from the surface, the density of majority spin electrons within the considered thickness decreases on a timescale up to $0.3 \mathrm{ps}$ and thus leads to demagnetization. This result was compared to experimental data obtained by probing the demagnetization via XMCD. 


\section{Ultrafast Spin Dynamics}
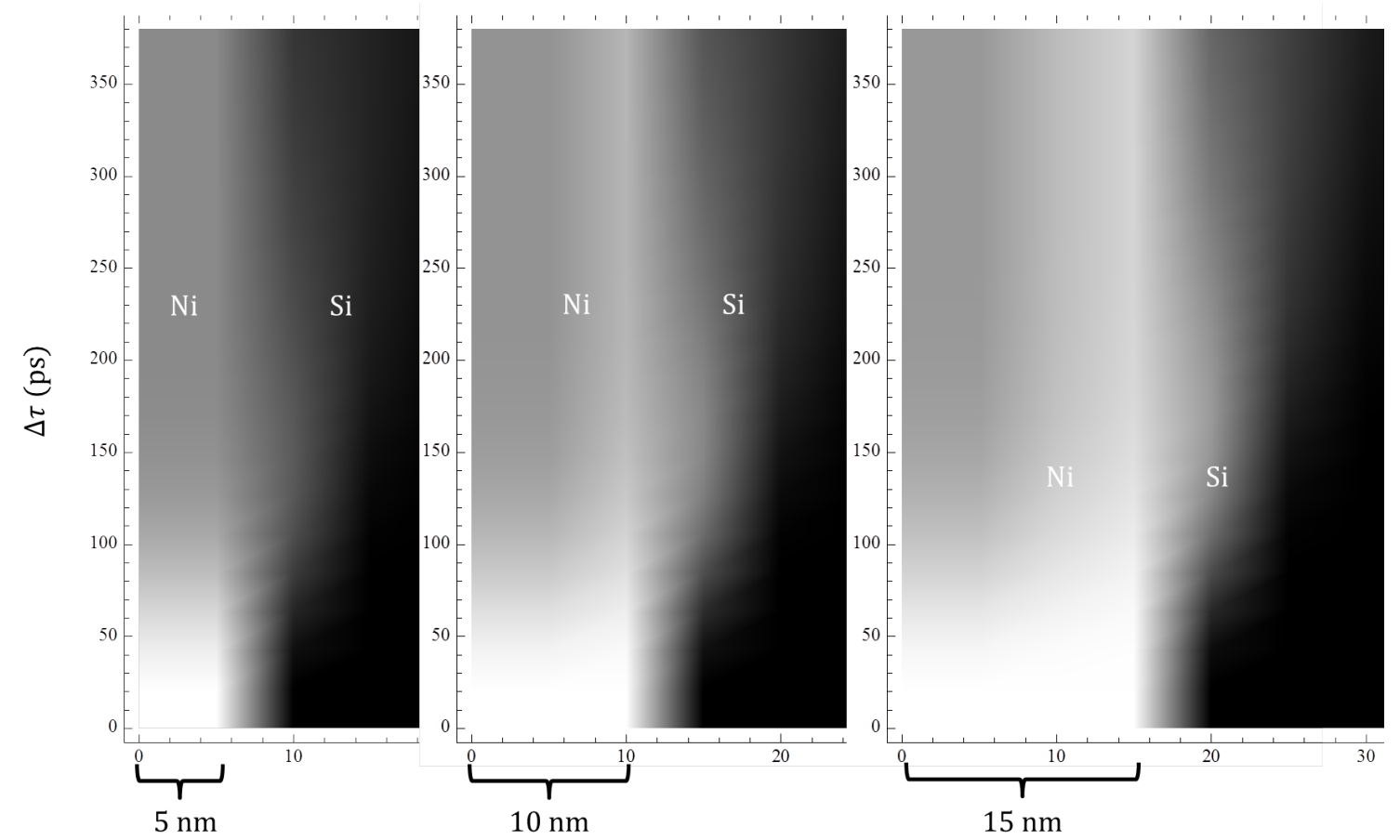

Figure 1.23: Color coded: Majority spin electron density, (white, high density, black, low density) plotted against the delay time $\Delta \tau$ and the $\mathrm{Ni}$ and $\mathrm{Si}$ depth, for the thicknesses $5 \mathrm{~nm}, 10 \mathrm{~nm}$ and $15 \mathrm{~nm}$. Taken from [9].

Figure 1.22 depicts the demagnetization times simulated based on the superdiffusive transport. These are the first simulations performed by M. Battiato [9] for different $\mathrm{Ni}$ film thicknesses and have not been published yet. It shows that while the nickel film demagnetizes, the surface of the Si substrate gains magnetization. While for the nickel films thicker than the penetration depth, the magnetization of the substrate decreases with increasing thickness, because the electrons from the surface have larger distances to reach the substrate and thus need more energy. For the thinner Ni films, apparently the majority spin electron density decreases, leading to a lower magnetization of the substrate.

Figure 1.23 shows the magnetization distribution in $z$-direction color coded (white, a high majority spin density and black, a low majority spin density) for the nickel films, $5 \mathrm{~nm}, 10 \mathrm{~nm}$ and $15 \mathrm{~nm}$. The both thicker Ni samples show a higher majority spin electron density than the $5 \mathrm{~nm} \mathrm{Ni}$ film at the first $5 \mathrm{~nm}$ of the Si surface. This is also an indication that there are more electrons moving from the thicker films into the substrate, than from the thinner film.

The Kerr rotation $\theta_{K}$ in Si is expected to be weak, because of low spin-orbit interaction. However, assuming a detectable $\theta_{K}$ from the Si substrate, the super-diffusive transport is a possible contribution to the saturation of demagnetization observed at high pump fluences in the thinner $2 \mathrm{~nm}$ and $5 \mathrm{~nm}$ Ni films. While the demagnetization in fact might be larger, the demagnetization mirrored in the measured 


\subsection{Experimental Results}

MOKE signal, saturates, because for the $5 \mathrm{~nm}$ Ni film only $30 \%$ of the measurement signal originates from the nickel film. The remaining signal are from reflections on the substrate, which according to the super-diffusive transport simulations gains magnetization, when the majority spin electrons move away from the nickel film. In average, the measured demagnetization remains constant. For the thicker samples, the contribution from the magnetization of the substrate to the MOKE signal reduces to $50 \%$ for the $10 \mathrm{~nm}$ nickel film, and $35 \%$ for the $15 \mathrm{~nm}$ nickel film. In the case of the $10 \mathrm{~nm}$ nickel film, this leads to larger measured demagnetization than in the thinner films.

This explanation is the result of first simulations. There are several issues that need to be resolved, before this explanation can be confirmed. The first issue is the exact amount of the contribution from the Si substrate to the MOKE signal. The calculations performed so far assume that the electrons, which supper-diffuse into the $\mathrm{Si}$ from the Ni film do not change their properties regarding the MOKE signal. Currently DFT calculations are performed, disentangling both contributions, the nickel's and the silicon's, to be able and compare the simulated to experimental data. Simulations for different pump fluences are necessary to check, whether the majority spin electron accumulation in the Si substrate surface increases with the fluence, or whether there is a saturation effect, which might be responsible e.g. for the saturating demagnetization observed in the two thinnest nickel films. Nevertheless, the super-diffusive transport seems to contribute to the demagnetization and should be treated as an additional mechanism contributing to ultrafast demagnetization in the analysis of such experiments. In this case, also experiments performed on thin nickel films deposited on transparent substrates like $\mathrm{MgO}$ might clarify, whether there is the possibility of another contribution to the MOKE signal than the nickel's when the films are deposited on Si. 


\subsection{Concluding Remarks}

The vivid field of ultrafast magnetization dynamics shows that the mechanisms responsible for demagnetization are not entirely disentangled yet. Both, the thermal LLB and the m3TM approaches, based on exchange interaction and spin disorder, show a wide agreement with experimental data for thin films.

The super-diffusive mechanism is worth consideration, because at first view it provides an explanation to the discrepancy between the measured high electron temperatures and the relatively low and saturating demagnetization of the thin $d=2 \mathrm{~nm}, 5 \mathrm{~nm}$ nickel films at high fluences.

\subsubsection{Summary}

The objective of the experiments discussed in the first part of this thesis has been to investigate, how distinct the features of the thermal model described by the LLB equation, which is derived starting with single spins in fluctuating Langevin fields, show in the experimental data. The analysis, fitting the analytical solution of the three temperature model extended by a second relaxation time $\tau_{M, r e}$ to the experimental data proves the validity of the thermal model. All the features predicted by this model namely, the critical slowing down of the demagnetization and the slow relaxation at high temperatures are exhibited in the experimental data. They can be quantified by the extracted relaxation times.

The analysis of the reflectivity data shows that the two temperature model is a suitable approach to experimentally determine the electron temperatures required as input parameters to model magnetization dynamics via the LLB equation. The temperatures extracted from reflectivity data for nickel film thicknesses around the penetration depth lead to excellent agreement between experimental and simulated magnetization dynamics data. For the magnetization dynamics of nickel films thinner than the penetration depth, the experimentally obtained electron temperatures do not provide reliable values to be used as input data into the simulations. Further work on this particular subject is necessary. Using more transparent substrates, e.g. $\mathrm{MgO}$, instead of Si might reduce the contribution of the substrate to the reflectivity signal, delivering more reliable electron temperature values. Applying shorter probe pulse wavelengths $(\lambda \approx 400 \mathrm{~nm})$ would reduce the penetration depth to $\Lambda_{\text {opt }} \approx 13 \mathrm{~nm}$. This is not enough to discard the signal contribution from the $\mathrm{Si}$ substrate for the thin nickel films. In addition to that, also Ni films thicker than $\Lambda_{\text {opt }}$ do not provide information about the electron temperature from reflectivity experiments. There, a different method needs to be implemented in order to gain insight in these processes. In that case, the change of the probe pulse wave length might help avoiding the sign change, exhibited in the data shown in figure B.12. 

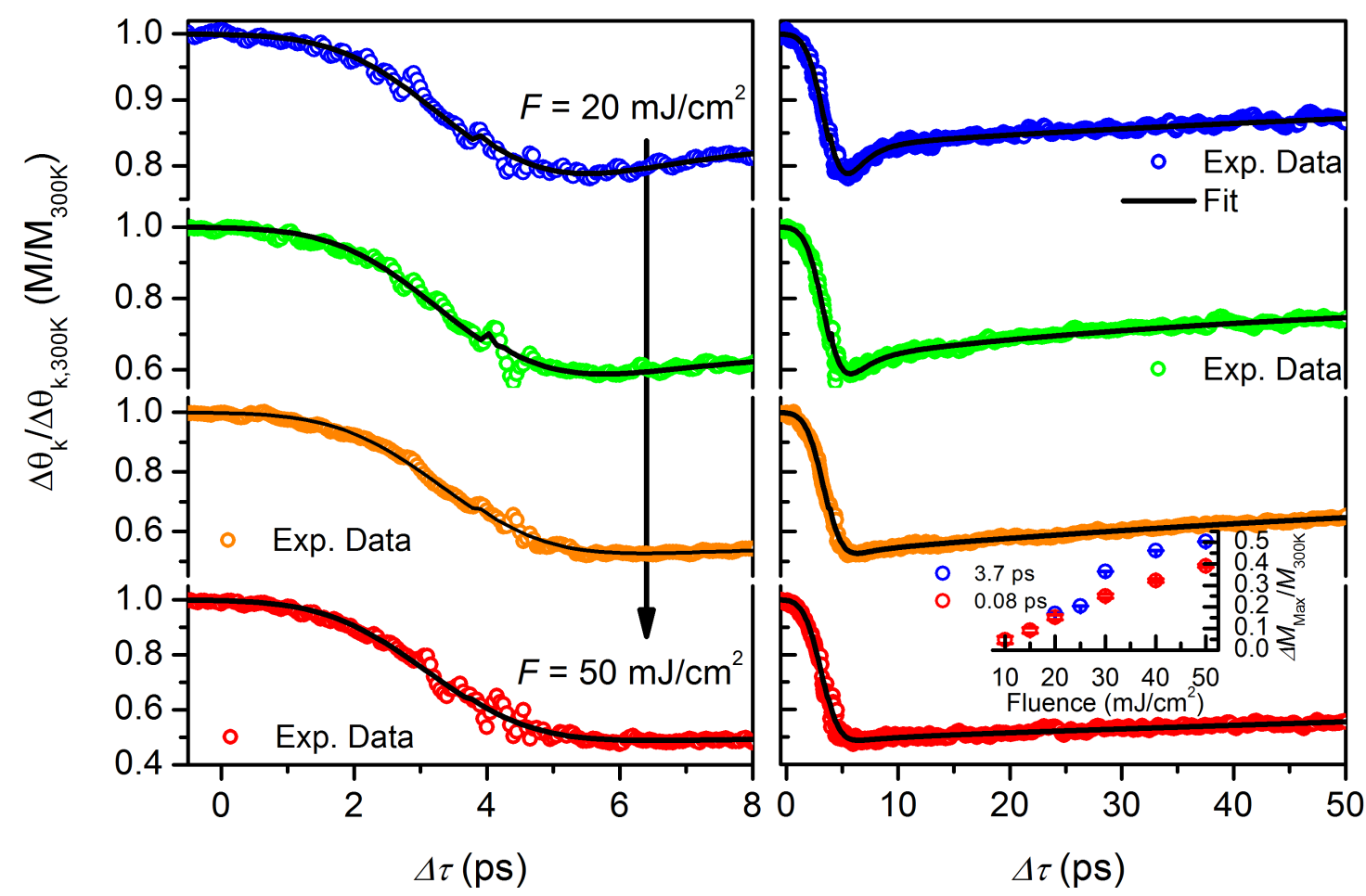

Figure 1.24: Demagnetization curves, measured applying pump pulses temporarily stretched to approximately $2.6 \mathrm{ps}$, on the $15 \mathrm{~nm} \mathrm{Ni}$ film, and fluences up to $50 \mathrm{~mJ} / \mathrm{cm}^{2}$. Inset: Maximum demagnetization from saturation magnetization at $300 \mathrm{~K}$, applying the stretched, and the $80 \mathrm{fs}$ pump pulse for comparison. Adapted from [44].

\subsubsection{Outlook}

Future experiments will help to disentangle the underlying mechanisms responsible for demagnetization as well as their relevance, which is currently under a vivid discussion. In a next step the magnetization dynamics can be triggered applying temporally stretched pump pulses around $1-4 \mathrm{ps}$. This will avoid the generation of hot electrons during excitation even at high pump fluences. The first results obtained in such experiments are plotted in figure 1.24. The graphs show magnetization dynamics spectra of a $15 \mathrm{~nm} \mathrm{Ni}$ film, triggered by pump pulses temporarily stretched to approximately $2.6 \mathrm{ps}$. In those experiments the same fluences were applied as in the previously presented experiments, where the magnetization dynamics was triggered by short excitation pulses [44]. The dynamics for the two lowest fluences $\left(10 \mathrm{~mJ} / \mathrm{cm}^{2}\right.$ and $\left.15 \mathrm{~mJ} / \mathrm{cm}^{2}\right)$ have not been measured, because the demagnetization is hardly detectable in this range. In those experiments, the maximum electron temperatures barely exceed $T_{C}$ at high fluences, making sure that there are no relevant contributions from hot electrons. At the same time, the maximum demagnetization is around $10 \%$ higher than in the experiments using short excitation pulses (see inset of figure 1.24). This outcome is supported by the findings 
in [38], stating that non-thermal electrons are not decisive for ultrafast demagnetization. However, these results are controversial to the latest ab-initio calculations presented in [15]. There the spin-flip probabilities were calculated using the energyand spin dependent Eliashberg function and for comparison the same probabilities were calculated using the Elliott approximation. This comparison is important, because the Elliott-Yafet electron-phonon spin-flip scattering so far is considered the main mechanism for ultrafast spin dissipation. Further, in [15] they argue that not the spin-flip probability is decisive for demagnetization, but the imbalance between the majority and minority subband occupation and that in the non-thermal state this imbalance leads to a higher spin-flip probability for majority spin electrons than the minority spin electrons, thus leading to a higher demagnetization rate than in the thermalized state, where the imbalance is smaller. They conclude that in the Elliott approximation the Elliott-Yafet phonon-mediated demagnetization is not high enough to explain ultrafast demagnetization in the thermalized state, but rather in the non-thermalized.

With this discussion just opened, the field leaves a lot of open questions to be clarified in further research. A further verification of the LLB model, in which now the quantum mechanic spins have been implemented [3], will follow shortly. So far, the spins have been treated semi-classically [6]. The comparison to experiments carried out using variable pump pulse lengths will show, which mechanisms are underlying the ultrafast spin-flips. Further experimental verification of the superdiffusive model is necessary, as well as the estimation of the Kerr rotation stemming from the electrons super-diffusing into the Si substrate, which is a very interesting mechanism for thermal spin injection by itself. 



\section{Magneto-Seebeck Effect in Tunnel Junctions}

\subsection{Thermoelectric Effects}

In 1821 Thomas Johann Seebeck, when working on galvanic chains discovered that heating one end of a metallic plate, which is connected in a closed loop with a copper coil induces a current. At that time, the current was measured by a compass needle placed along the coil. An electric current through the coil induces a magnetic field and forces the compass needle to turn from its original direction. The force on the needle is proportional to the current through the coil. This phenomenon was only shortly discovered by Hans Christian Oersted in 1819.

By holding one end of a metallic plate (bismuth, antimony) at a constant temperature in iced water and heating the other, he was also able to discover that the current through the coil was increasing with the temperature difference between both ends of the plate. The electric current measured using the copper coil stemmed from the voltage generated by the temperature gradient through the metallic plates [55].

The Seebeck effect is the first thermoelectric effect, which has been discovered. Several others followed. Recently, a lot of new interest emerged from these effects in combination with magnetism. The development of conventional electronics seems to have reached an end and is threatened by a breakdown of Moore's law, as with smaller device sizes current densities increase. Therefore, combining magnetism with thermoelectric effects seems to be inevitable to build an expandable foundation for new control systems of logic and memory devices.

In this chapter, thermoelectric effects are introduced, starting with the derivation of a completed concept to describe the thermoelectric and thermomagnetic effects. These phenomena of simultaneous heat flow and electric current in one system require the formalism of irreversible thermodynamics as a tool. The derivation for the description of the thermoelectric effects follows the steps in [14, Chapter 16,17]. A supplementary inclusion of magnetic currents into this description is given, according to [36]. Following that, the effects are explained from a phenomenological point of view for a clearer understanding of the presented experiments.

The construction and features of the experimental setup to measure the magnetoSeebeck effect in magnetic tunnel junctions by generating the temperature gradient optically are presented. The experimental data is discussed in the view of ab-initio calculations of the magneto-Seebeck effect in MgO-based magnetic tunnel junctions. 


\subsection{Irreversible Thermodynamics and Thermodynamic Transport}

Following the derivation in [14], a system consisting of $k$ discrete subsystems described by the extensive parameters $X_{k}$ is considered. The extensive parameter of the entire system $X$ is a conserved quantity, given by:

$$
\sum_{k} X_{k}=X=\text { constant. }
$$

In a simple case of $k=2$, the equilibrium values of the extensive parameters if they are unconstrained, can be calculated from:

$$
A F_{12} \equiv\left(\frac{\partial S}{\partial X_{1}}\right)_{X}=\left(\frac{\partial\left(S_{1}+S_{2}\right)}{\partial X_{1}}\right)_{X}=\frac{\partial S_{1}}{\partial X_{1}}-\frac{\partial S_{2}}{\partial X_{2}}=F_{1}-F_{2},
$$

given that:

$$
\frac{\partial S_{2}}{\partial X_{1}}=\frac{\partial S_{2}}{\partial\left(X-X_{2}\right)}=0-\frac{\partial S_{2}}{\partial X_{1}}
$$

The two possible cases are: First, if $A F_{12}=0$, then the system is in equilibrium. Second, if $A F_{12} \neq 0$, then an irreversible process occurs. In general, the parameter $A F_{k i}$ acts as a generalized force from system $k$ to system $i$, and is called "affinity". The parameters $F_{1}$ and $F_{2}$ are the local intensive parameters ${ }^{1}$. Their difference determines the strength of the acting force. The simplest example to illustrate this concept is by considering two systems at different temperatures $T_{1}$ and $T_{2}$, separated by a diathermal wall. In the case of an isolated system without particle exchange, the extensive parameter is the inner energy $U$, with $d U=T d S$. The affinity for the exchange of heat is $A F_{12}=F_{1}-F_{2}=\frac{1}{T_{1}}-\frac{1}{T_{2}}$. If the two systems are at the same temperature, $T_{1}=T_{2}$, no heat is transferred from one system to the other. This is the equilibrium case. If $T_{1} \neq T_{2}$, the difference of the inverse temperatures acts as the generalized force, driving the heat from the warmer to the colder system, until the temperatures are equal.

The flux $J_{k}$ that describes the rate of change of $X_{k}$, as a response to the applied force, is defined by:

$$
J_{k}=\frac{d X_{k}}{d t}
$$

That concludes that a flux is generated by a nonzero affinity if the intensive parameters of the subsystems are not in equilibrium.

A more convenient way of identifying the single affinities in a particular system is by the rate of entropy production for the entire system $S\left(X_{0}, X_{1}, \ldots\right)$ with respect to time:

$$
\dot{S}=\frac{d S}{d t}=\sum_{k} \frac{\partial S}{\partial X_{k}} \frac{d X_{k}}{d t}=\sum_{k, i} A F_{k i} \cdot J_{k}
$$

\footnotetext{
${ }^{1}$ Reminder: Extensive parameters depend on the amount of the examined system, while intensive parameters maintain their values if the system is e.g. divided into two parts.
} 
Dealing with thermoelectric effects, e.g. electrons moving through a wire exposed to a temperature gradient, a description of irreversible processes in continuous three dimensional systems is required, rather than the exchange of the component $k$ between two discrete systems. For this purpose, the rates of change of $X_{k}$ are extended into three dimensions, where the fluxes are components $J_{k x}, J_{k y}, J_{k z}$ in three directions, of the current density vector $\boldsymbol{J}_{k}$. The current density describes the amount of the component $k$ moving through an unit area by unit time, i.e. a non-equilibrium system. This makes it difficult to describe the systems by equilibrium parameters like the entropy. Therefore, the rate of entropy production equation 2.1 needs to be adapted for such continuous non-equilibrium systems, by introducing a local entropy $S\left(X_{0}, X_{1}, \ldots\right)$ for an infinitesimally small region, depending on local extensive parameters:

$$
d S=\sum_{k} F_{k} d X_{k}, \quad \text { or per unit volume : } d s=\sum_{k} F_{k} d x_{k},
$$

with the local intensive parameters $F_{k}$ being the same functions of the local extensive parameters, as if the system would be in equilibrium. This makes it possible to describe the continuous variation of temperature along a wire by thermostatics, despite the implication of temperature existence only in the equilibrium state.

On the basis of equation 2.2, an entropy current density, which describes the entropy transported through the unit area per unit time, can be defined:

$$
\boldsymbol{J}_{S}=\sum_{k} F_{k} \cdot \boldsymbol{J}_{k},
$$

where $\boldsymbol{J}_{k}$ is the current density of the extensive parameter $X_{k}$. Using the equation of continuity, the rate of entropy production per unit volume can be described by:

$$
\dot{s}=\frac{\partial s}{\partial t}+\nabla \cdot \boldsymbol{J}_{S}
$$

where the first term describes the increase of local entropy per unit volume. Since the extensive parameters are conserved quantities, the continuity equation yields:

$$
0=\frac{\partial x_{k}}{\partial t}+\nabla \cdot \boldsymbol{J}_{k}
$$

Based on equation 2.4, the analogous to equation 2.1 in three dimensions for continuous systems can be derived. The first term in equation 2.4 results from the right part of equation 2.2 $\frac{\partial s}{\partial t}=\sum_{k} F_{k} \frac{\partial x_{k}}{\partial t}$. The divergence of $\boldsymbol{J}_{S}$ from equation 2.3 is given by:

$$
\nabla \cdot \boldsymbol{J}_{S}=\nabla \cdot\left(\sum_{k} F_{k} \boldsymbol{J}_{k}\right)=\sum_{k}\left(\nabla F_{k} \cdot \boldsymbol{J}_{k}+F_{k} \nabla \cdot \boldsymbol{J}_{k}\right) .
$$

Comparing the components to those in equation 2.5, yields:

$$
\dot{s}=\sum_{k}(F_{k} \underbrace{\frac{\partial x_{k}}{\partial t}}_{-\nabla \cdot \boldsymbol{J}_{k}}+\nabla F_{k} \cdot \boldsymbol{J}_{k}+F_{k} \cdot \nabla \boldsymbol{J}_{k})=\sum_{k} \nabla F_{k} \cdot \boldsymbol{J}_{k} .
$$




\subsection{Irreversible Thermodynamics and Thermodynamic Transport}

Both equations 2.1 and 2.6 differ by their affinity parameters. In the case of discrete systems, equation 2.1. the affinity is defined as the 'difference in the entropyrepresentation intensive parameters'. For continuous systems (equation 2.6) it is the 'gradient of entropy-representation intensive parameters' [14].

In the last step, a detailed analysis of the fluxes brings them into a form that simplifies their further handling. In this context the term "Markoffian" is introduced. In Markoffian systems, the flux at a given time $t=t_{1}$ depends only on the affinities at that particular time, but not on affinities at other times, e.g. at $t_{2}$, i.e. $J_{k}=$ $J_{k}\left(A F_{k}\left(t=t_{1}\right)\right) \neq J_{k}\left(A F_{k}\left(t=t_{2}\right)\right)$. In Non-Markoffian systems the opposite is true. The flux at a given time $t=t_{1}$ can also depend on values of affinities at times $t \neq t_{1}$. This concludes that non-Markoffian systems have a memory. Additionally, in Markoffian systems, the flux $J_{k}$ not only depends on its own affinity $A F_{k}$, and its intensive parameter $F_{k}$, but on all affinities and intensive parameters in the system, at that given time:

$$
J_{k}=J_{k}\left(A F_{0}, A F_{1}, \ldots, A F_{j}, \ldots, F_{0}, F_{1}, \ldots, F_{j}, \ldots\right),
$$

given that the indices denoting the vector components are dropped. Although there is a dependence of the flux on other affinities, the strongest is on its own, and the flux vanishes if the affinities vanish. Therefore, a Taylor expansion of $J_{k}$ can be made dropping the constant term:

$$
J_{k}=\sum_{j} L_{j k} A F_{j}+\frac{1}{2 !} \sum_{i} \sum_{j} L_{i j k} A F_{i} A F_{j}+\ldots
$$

with the first order $L_{j k}$ and second order $L_{i j k}$ kinetic coefficients:

$$
L_{j k}=\left(\frac{\partial J_{k}}{\partial A F_{j}}\right)_{0}, \quad L_{i j k}=\left(\frac{\partial^{2} J_{k}}{\partial A F_{i} \partial A F_{j}}\right)_{0} .
$$

The first order kinetic coefficients are functions of the local intensive parameters, $L_{j k}=L_{j k}\left(F_{0}, F_{1}, \ldots\right)$. A similar definition holds for the higher order kinetic coefficients. For the purpose of thermally induced electron motion in metals, the restriction to the linear terms is sufficient, because the gradients of the local affinities $\nabla F_{k}$ are of the form $\nabla \frac{1}{T}$. Besides that, for small changes in $T$, higher order terms do not have any significance in the flux. Therefore, the higher order kinetic coefficients will be dropped in the following calculations. For later calculation very convenient is the property of the first order kinetic coefficients expressed by the Onsager reciprocal theorem:

$$
L_{j k}=L_{k j}
$$

This relation means, there is a linear effect of the $j$ th affinity on the $k$ th flux and of the $k$ th affinity on the $j$ th flux. A detailed explanation of the Onsager theorem is given in [13, 48] or [14, Chapter 16]. 


\subsubsection{The Dynamical Equations and Kinetic Coefficients}

Employing the tools obtained in the previous section, the dynamical equations together with their kinetic coefficients can be determined for a one dimensional metal wire, as follows. Starting with the local entropy density $s$ :

$$
d s=\frac{1}{T} d u-\frac{\mu}{T} d n-\sum_{k}\left(\frac{\mu_{k}}{T}\right) d n_{k},
$$

where $u$ is the local energy density, $\mu$ is the electro-chemical potential (per particle) of electrons, $n$ is the number of electrons per unit volume. The second term on the right hand side describes the considered electron, and the sum collects all the contributions to the entropy of other atoms building up the solid. The current densities for these extensive parameter densities follow from the definition in equation 2.3

$$
\boldsymbol{J}_{S}=\frac{1}{T} \cdot \boldsymbol{J}_{U}-\frac{\mu}{T} \cdot \boldsymbol{J}_{N}
$$

with the current density of entropy $\boldsymbol{J}_{S}$, the current density of energy $\boldsymbol{J}_{U}$ and the current density of the number of electrons $\boldsymbol{J}_{N}$. The contributions of the sum in equation 2.9 are dropped due to the approximations made in equation 2.7. Having the current densities, and using the continuity equations for these current densities, the rate of production of local entropy yields:

$$
\dot{s}=\nabla \frac{1}{T} \cdot \boldsymbol{J}_{U}-\nabla \frac{\mu}{T} \cdot \boldsymbol{J}_{N} .
$$

Instead of $\boldsymbol{J}_{U}$, the current density of heat is of general interest. It is related to entropy through $d Q=T d S$, leading to:

$$
\boldsymbol{J}_{Q}=T \boldsymbol{J}_{S} \Rightarrow \boldsymbol{J}_{Q}=\boldsymbol{J}_{U}-\mu \boldsymbol{J}_{N}
$$

This allows the elimination of $\boldsymbol{J}_{U}$ from the production rate of entropy equation:

$$
\dot{s}=\nabla \frac{1}{T} \cdot \boldsymbol{J}_{Q}-\frac{1}{T} \nabla \mu \cdot \boldsymbol{J}_{N} .
$$

Finally, for one dimensional problems it is sufficient to drop the current densities $\boldsymbol{J}_{Q}$ and $-\boldsymbol{J}_{N}$ and use one single component from each, the fluxes $J_{Q}$ and $-J_{N}$. $\nabla \frac{1}{T}$ and $\frac{1}{T} \nabla \mu$ are the associated affinities, according to equation 2.6. With the approximation in equation 2.7, this leads to the following one dimensional dynamic equations:

$$
\begin{aligned}
-J_{N} & =L_{11} \frac{1}{T} \nabla \mu+L_{12} \nabla \frac{1}{T} \\
J_{Q} & =L_{21} \frac{1}{T} \nabla \mu+L_{22} \nabla \frac{1}{T} .
\end{aligned}
$$

Making use of the Onsager relation (equation 2.8) $L_{12}=L_{21}$, reduces the number of coefficients to be determined to three. 


\subsection{Irreversible Thermodynamics and Thermodynamic Transport}

In the last step, the kinetic coefficients from the dynamical equations 2.11 need to be determined by defining the conductivities. For this purpose, first the electrochemical potential $\mu$ can be considered as the sum of a chemical part $\mu_{c}$ and an electrical part $\mu_{e}$. With the chemical part depending on the temperature and the concentration and the electrical part being $\mu_{e}=e \Phi$, the product of the charge of an electron and the ordinary electrostatic potential. The chemical part of the gradient $1 / e \nabla \mu_{c}$ makes the field, actually the driving force, arising from the concentration gradient. The electrical part $1 / e \nabla \mu_{e}$ is the electric field $E$. The electric conductivity is defined as:

$$
\sigma=\frac{-e J_{N}}{E}=\frac{-e J_{N}}{\frac{1}{e} \nabla \mu_{e}}
$$

The minus sign stems from the negative charge of the electron. In the present work, both, the current density $\boldsymbol{J}_{N}$ or the flux $J_{N}$ of particles were defined without taking into account the charge properties of the considered particles. Therefore, if describing the flow of electrons, which is usually done by the current density $\vec{\jmath}$ in most textbooks, the current density or flux has to be multiplied by the charge, $\vec{\jmath}=-e \boldsymbol{J}_{N}$, or $j=-e J_{N}$. The total electric field is then given by $\mathcal{E}=E+1 / e \nabla \mu_{c}$. The definition given for $\sigma$ is valid for systems exposed to isothermal conditions $(\nabla T=0)$, then also $\nabla \mu_{c}=0$ and the first equation 2.11 determines the first kinetic coefficient $L_{11}$ :

$$
\sigma \equiv \frac{e^{2} L_{11}}{T}, \quad \Rightarrow \quad L_{11}=\frac{\sigma T}{e^{2}} .
$$

Second, the heat conductivity is given by the heat flux per unit temperature gradient, if there is no electric current, $J_{N}=0$ :

$$
\kappa \equiv \frac{-J_{Q}}{\nabla T}
$$

This gives the following relations for the remaining kinetic coefficients:

$$
L_{12}=L_{21}=L_{11} T \frac{\nabla \mu}{\nabla T}, \quad L_{22}=\kappa T^{2}+\left(T \frac{\nabla \mu}{\nabla T}\right)^{2} L_{11}
$$

The above derived dynamic equations 2.11 and their kinetic coefficients given in equations (2.12 2.13) build the basis for the description of the three thermoelectric effects, outlined below.

\subsubsection{The Seebeck Effect}

The Seebeck effect occurs, when an electromotive force is produced in a thermocouple and no electric current is present. Considering a thermocouple which consists of two wires $A$ and $B$ with two junctions: One at $T_{1}, \mu_{1}$, the other at $T_{2}, \mu_{2}$ and $T_{2}>T_{1}$, as illustrated in figure 2.1. The connecting wires leading to the voltmeter are at the temperature $T^{\prime}$ and the voltmeter itself is a perfect resistor for the electric current, but a perfect conductor for the heat current. 


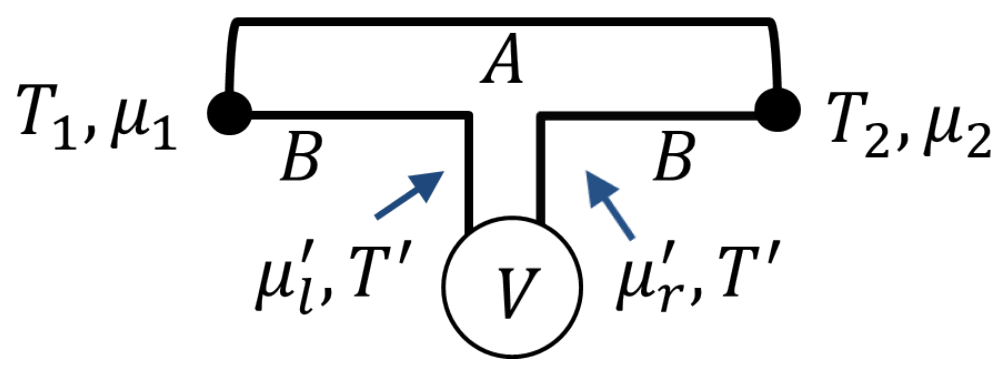

Figure 2.1: A thermocouple consisting of two wires $A$ and $B$ connected at points of temperature and electrochemical potential $T_{1}, \mu_{1}$ and $T_{2}, \mu_{2}$ respectively. The wires leading to the voltmeter are at the same temperature $T^{\prime}$. The voltmeter has a perfect electric resistivity of $\infty \Omega$, but has zero resistivity for the heat current.

Because of the voltmeter being a perfect resistor, $J_{N}=0$, the electrochemical potential can be calculated from the first kinetic equation 2.11 by applying the chain rule $\nabla 1 / T=-1 / T^{2} \nabla T$ :

$$
\nabla \mu=\frac{L_{12}}{T L_{11}} \nabla T
$$

Integrating equation 2.14 around the thermocouple gives the following contributions:

$$
\begin{aligned}
\mu_{2}-\mu_{1} & =\int_{1}^{2} \frac{L_{12}^{A}}{T L_{11}^{A}} d T \\
\mu_{2}-\mu_{r}^{\prime} & =\int_{r}^{2} \frac{L_{12}^{B}}{T L_{11}^{B}} d T \\
\mu_{l}^{\prime}-\mu_{1} & =\int_{1}^{l} \frac{L_{12}^{B}}{T L_{11}^{B}} d T .
\end{aligned}
$$

The electro chemical potentials at the interfaces of both wires $\mu_{1}$ and $\mu_{2}$ can be eliminated by rearranging the first two equations to put $\mu_{2}$ on one side, and by rearranging the last equation to put $\mu_{1}$ on one side. Substituting those parameters, leads to:

$$
\int_{r}^{2} \frac{L_{12}^{B}}{T L_{11}^{B}} d T+\mu_{r}^{\prime}=\int_{1}^{2} \frac{L_{12}^{A}}{T L_{11}^{A}} d T-\int_{1}^{l} \frac{L_{12}^{B}}{T L_{11}^{B}} d T+\mu_{l}^{\prime}
$$

Rearranging once again leads to the electro-chemical potential at the voltmeter.

$$
\mu_{r}^{\prime}-\mu_{l}^{\prime}=\int_{1}^{2}\left(\frac{L_{12}^{A}}{T L_{11}^{A}}-\frac{L_{12}^{B}}{T L_{11}^{A}}\right) d T .
$$

With the fact that the wires connecting the voltmeter are at the same temperature, the Seebeck voltage is:

$$
V_{S}^{1 \rightarrow 2}=\frac{1}{e}\left(\mu_{r}^{\prime}-\mu_{l}^{\prime}\right)=\int_{1}^{2}\left(\frac{L_{12}^{A}}{e T L_{11}^{A}}-\frac{L_{12}^{B}}{e T L_{11}^{B}}\right) d T .
$$




\subsection{Irreversible Thermodynamics and Thermodynamic Transport}

The Seebeck coefficient or thermoelectric power ${ }^{2}$ of the thermocouple is $Q_{\mathrm{S}_{\mathrm{AB}}}$ is defined by the change of voltage per temperature difference. The sign of $Q_{\mathrm{S}_{\mathrm{AB}}}$ is defined to be positive, when the resulting voltage drives the current from wire $A$ to wire $B$ at the point with the higher temperature $T_{2}$ :

$$
Q_{\mathrm{S}_{\mathrm{AB}}}=\frac{\partial V_{S}^{1 \rightarrow 2}}{\partial T_{2}}=\left(\frac{-L_{12}^{B}}{e T L_{11}^{B}}\right)-\left(\frac{-L_{12}^{A}}{e T L_{11}^{A}}\right)=Q_{\mathrm{S}_{\mathrm{A}}}-Q_{\mathrm{S}_{\mathrm{B}}}
$$

where $Q_{\mathrm{S}_{\mathrm{A}}}$ and $Q_{\mathrm{S}_{\mathrm{B}}}$ are the Seebeck coefficients of the single wires $A$ and $B$. That means, in general the Seebeck coefficient is defined as:

$$
Q_{\mathrm{S}}=\frac{-L_{12}}{e T L_{11}}
$$

In this way, the dynamic equations 2.11 can be rewritten with the physical properties conductivity $\sigma$, the thermal heat conductivity $\kappa$ and the thermoelectric power or Seebeck coefficient $Q_{\mathrm{S}}$ :

$$
\begin{aligned}
-J_{N} & =\frac{T \sigma}{e^{2}} \frac{1}{T} \nabla \mu-\frac{T^{2} \sigma Q_{\mathrm{S}}}{e} \nabla \frac{1}{T} \\
J_{Q} & =\frac{T^{2} \sigma Q_{\mathrm{S}}}{e} \frac{1}{T} \nabla \mu+\left(T^{3} \sigma Q_{\mathrm{S}}^{2}+T^{2} \kappa\right) \nabla \frac{1}{T} .
\end{aligned}
$$

One application of the Seebeck effect is to keep one joint of a thermo-couple at a fixed, known temperature, and use the other as a probe to measure the difference in temperature. If calibrated, temperature can be directly calculated from the voltage generated by the temperature difference between both joints.

\subsubsection{The Peltier Effect}

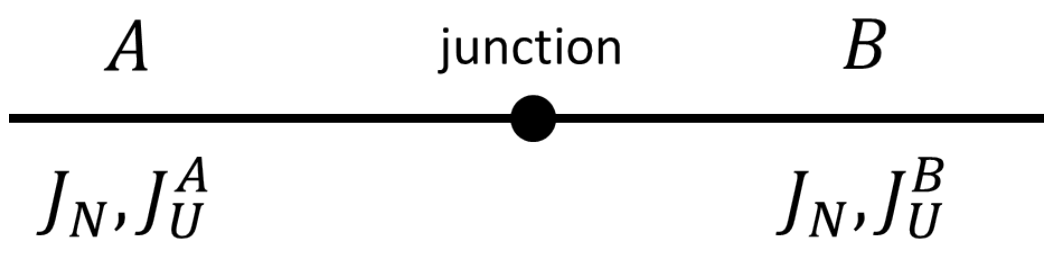

Figure 2.2: Two wires at the same temperature connected through a junction. The electric current $e \boldsymbol{J}_{N}$ causes a heat flow through the junction.

The Peltier effect depicts the opposite situation of the Seebeck effect, by describing the heat evolution across an isotherm junction of two wires $A$ and $B$ (different materials) exposed to an electric current $\vec{\jmath}=-e \boldsymbol{J}_{N}$, sketched in figure 2.2. Across

\footnotetext{
${ }^{2}$ The Seebeck coefficient and thermoelectric power refer to the same parameter and can be used synonymously. Later, these terms will be extended to magneto-Seebeck coefficient and magnetothermoelectric power, also referring to the same parameters and can be used synonymously.
} 
the junction, the flow of electrons $J_{N}$ and the electrochemical potential $\mu$ will be continuous, since there cannot be a different number of electrons behind the junction, than in front of it. The continuity of $\mu$ arises from the fact that there is a junction. However, the total energy flow $J_{U}$ across the junction will be discontinuous that means, total energy will be stored around the junction. Therefore, there has to be a discontinuity in the heat flow, since the total energy flow is given by $J_{U}=J_{Q}+\mu J_{N}$, with $J_{U}^{A}-J_{U}^{B}=J_{Q}^{A}-J_{Q}^{B}$. Considering the isothermal condition, the dynamic equations 2.17 in either wire give a heat flow of:

$$
J_{Q}=T Q_{\mathrm{S}} \cdot\left(e J_{N}\right)=Q_{\mathrm{P}} \cdot\left(e J_{N}\right) .
$$

Putting it into a slightly different expression, yields to:

$$
Q_{\mathrm{P}}^{\mathrm{AB}}=\frac{J_{Q}^{B}-J_{Q}^{A}}{e J_{N}}=T\left(Q_{\mathrm{S}}^{B}-Q_{\mathrm{S}}^{A}\right) .
$$

That means, the Peltier coefficient $Q_{\mathrm{P}}^{\mathrm{AB}}$ expresses the amount of heat that is supplied to the junction when the electric current $e J_{N}$ passes from wire $A$ to wire $B$.

A negative Peltier coefficient means that heat is transported away from the junction, therefore Peltier elements can be used for cooling. They are applied in small devices where efficiency is not most important, e.g. in diode lasers, CCD-Chips used for long exposure times or other mainly electronic devices.

\subsubsection{The Thomson Effect}

The Thomson effect combines both previously mentioned effects, as it describes the evolution of heat across a wire, which is exposed to a temperature gradient, as electric current passes through. If an electric conductor is exposed to a heat current $J_{Q} \neq 0$, but no electric current $\left(J_{N}=0\right)$, a temperature gradient will spread according to the kinetic coefficients of the conductor material. Placing this conductor in a heat bath, such that the temperature of the heat bath is the same as the conductor's at each point of contact, turning on an electric current through the conductor $\left(J_{N} \neq 0\right)$ will, according to the Peltier effect change the temperature of the conductor, which will be interchanged with the heat bath. This exchange consists of two parts: The Joule heat from the electric current and the Thomson heat from the temperature gradient of the reservoir. Since there is an electric current passing through the conductor, the total energy flow is interchanged with the heat reservoirs. The gradient of the total energy flow is:

$$
\nabla \cdot \boldsymbol{J}_{U}=\nabla \cdot\left(\boldsymbol{J}_{Q}+\mu \boldsymbol{J}_{N}\right) .
$$

Using the dynamical equations 2.17, $J_{Q}$ can be expressed in terms of $J_{N}$ as $J_{Q}=$ $T Q_{\mathrm{S}} e J_{N}+T^{2} \kappa \nabla 1 / T$, and expressing $\nabla \mu$ in terms of the first equation 2.17, gives:

$$
\nabla \cdot \boldsymbol{J}_{U}=T \nabla Q_{\mathrm{S}} \cdot(e \boldsymbol{J})_{N}+\nabla \cdot\left(T^{2} \kappa \nabla \frac{1}{T}\right)-\frac{e^{2}}{\sigma} \boldsymbol{J}_{N}^{2} .
$$




\subsection{Irreversible Thermodynamics and Thermodynamic Transport}

The second term in the equation above vanishes, assuming that the temperature distribution is given by the steady state without any electric current. In that state there is no change in the total energy flow. In that case $\nabla \cdot \boldsymbol{J}_{U}=0$ and $\boldsymbol{J}_{N}=0$ that also leaves the second term to be zero. As a consequence, the total heat flow is given by:

$$
\nabla \cdot \boldsymbol{J}_{U}=T \nabla Q_{\mathrm{S}} \cdot\left(e \boldsymbol{J}_{N}\right)-\frac{1}{\sigma}\left(e^{2} \boldsymbol{J}_{N}^{2}\right) .
$$

Finally, considering the Seebeck coefficient $Q_{\mathrm{S}}$ as a function of the local temperature and its gradient, $\nabla Q_{\mathrm{S}}=\frac{d Q_{\mathrm{S}}}{d T} \nabla T$, and substituting $\nabla Q_{\mathrm{S}}$ in equation 2.18 gives the final equation for the change of total energy flow:

$$
\nabla \cdot \boldsymbol{J}_{U}=T \frac{d Q_{\mathrm{S}}}{d T} \nabla T \cdot\left(e \boldsymbol{J}_{N}\right)-\frac{1}{\sigma}\left(e^{2} \boldsymbol{J}_{N}^{2}\right)
$$

The second term expresses the Joule heat. Due to the quadratic term it is independent of the direction of the electron flow. Heat is produced even if there is no temperature gradient, similar to the Peltier effect. The first term represents the Thomson heat. This is the heat, which is absorbed by the heat reservoirs when the current $\vec{\jmath}=-e \boldsymbol{J}_{N}$ passes through the temperature gradient $\nabla T$. The Thomson coefficient $Q_{\mathrm{T}}$ is thus defined by:

$$
Q_{\mathrm{T}}=\frac{\text { Thomson heat }}{\nabla T \cdot\left(e \boldsymbol{J}_{N}\right)}=T \frac{d Q_{\mathrm{S}}}{d T} .
$$

\subsubsection{Thermo-magneto-electric Dynamical Equations}

Johnson and Silsbee [36] extended the dynamical equations by including a magnetic flux already in 1987. They interpreted a spin-injection experiment, in which a spin polarized current from a ferromagnet is injected into a non-magnetic metal. In this experiment, the spin diffusion length in the non-magnetic metal is given by $\delta_{S}=\sqrt{2 D \tau_{D}}$, where $D$ is the diffusion constant. The spin relaxation time $\tau_{D}$ can be determined from measurements of $\delta_{S}$. In the non-magnetic metal, the spin polarization decays exponentially along the propagation direction on the length $s$. This decay can be analyzed by a spin detector [35].

In a thermodynamic system with spin-up and spin-down symmetry, in which heat and charge can flow homogeneously from one subsystem to the other, each charge carrier is additionally ascribed a magnetic moment $\mu_{B}$. The equilibrium state in the paramagnetic metal is considered, when all moments are randomly oriented and an equal number of spin-up and spin-down spins exists.

The current exchanged between the ferromagnet and the paramagnetic metal through the interface, is spin polarized. That means, the fraction $P$ of the electrons are carrying the majority spin of the ferromagnet, and the spin symmetry is broken. In addition to charge flow, also a flow of magnetic moment $I_{M}$ through the interface is present. In the considered case of two discrete systems, $I_{M}$ is a vector pointing in the direction of the magnetization, which points in a fixed direction, enabling $I_{M}$ to 
be treated as a scalar. Moreover, a net magnetic moment $\mathcal{M}$ extensively describes the state of the system in the paramagnet as current is flowing. The inclusion of the net magnetic moment as an extensive parameter requires some reconsideration before the inclusion into the dynamical equations. It is not in general a conserved quantity, and is connected to the magnetization flow by $\mathcal{M}=I_{M} \cdot \tau_{D}$. That means, that $\mathcal{M}$ can be either destroyed by spin relaxation after the time $\tau_{D}$, or created by spin scattering events in a ferromagnet, where an unpolarized electron can be scattered into a polarized band, characterized by the time $\tau_{f}$. If $\mathcal{M} \neq 0$, the system is considered out of equilibrium, having a non-equilibrium magnetization. The change of $\mathcal{M}$ can have two origins:

$$
d \mathcal{M}=d \mathcal{M}_{i}+d \mathcal{M}_{e}
$$

where $d \mathcal{M}_{i}$ is the change due to spin relaxation, which is an internal transfer, and $\mathcal{M}_{e}$ is an externally caused change, by transferring magnetic moment into the system. $\mathcal{M} \neq 0$ can only be preserved, if $\tau_{D} \rightarrow \infty$, then $d \mathcal{M}_{i}=0$. For the sake of simplicity, thin single domain films with a fixed volume and an external field parallel to the film plane are considered, in order to avoid demagnetization fields. The system is chosen such that the magnetic part of the internal energy $U_{H}$ in an applied external field $H$ is given by:

$$
U_{H}=-H \sum_{i} \mu_{i}=-H \mathcal{M}
$$

For a more convenient description of the changes in $\mathcal{M}$ with Taylor expansions, the parameter $\tilde{\mathcal{M}}$, the net magnetic moment's reference state is introduced. This makes two cases possible: First, a paramagnet with $\tilde{\mathcal{M}}=\mathcal{M}$. Second, a ferromagnet with a high magnetic moment $\mathcal{M}_{0}$ in zero external field, $\tilde{\mathcal{M}}=\mathcal{M}-\mathcal{M}_{0}$.

Following the procedure in the previous sections, the generalized forces have to be identified and an expression for the production rate of entropy needs to be derived. Starting with the first law of thermodynamics:

$$
d U=d Q-d W
$$

the change of internal energy is the heat added minus the work performed. The work performed splits into two contributions $d W=d W_{1}+d W_{2}$. The first contribution is the same as in the thermoelectric effects, $d W_{1}=\mu d N=-V d q$, the number of particles $N$ moved by the force of an electro-chemical potential $\mu$ or explicitly for the case of electrons, the number of charges $q$ moved by a voltage $V$. The second contribution needs to be examined more thoroughly. For the changes in $\mathcal{M}$ from internal processes $d \mathcal{M}_{i}$ due to spin relaxation, no analog process in the electrical case exists. External transfer of magnetization $d \mathcal{M}_{e}$ from or into the system depends on the magnetization potential. According to the definition of the internal magnetic energy $U_{H}$ no work can be associated with the change of the magnetic moment due to internal processes, but with the change of the external field, given by $\mathcal{M} d H$. Transferring magnetic moment into the system is given by $-H^{*} d \mathcal{M}_{e}$, in which the 


\subsection{Irreversible Thermodynamics and Thermodynamic Transport}

magnetic potential $-H^{*}$ will be determined from the free energy $F$. The first law of thermodynamics can be extended to:

$$
d U=d Q-\mathcal{M} d H-H^{*} d \mathcal{M}_{e}+V d q .
$$

With the free energy being $F=U-T S$, the change in the free energy becomes:

$$
d F=d Q-T d S-S d T-\mathcal{M} d H-H^{*} d \mathcal{M}_{e}+V d q .
$$

For reversible processes, $d Q=T d S$ and the change in free energy simplifies to:

$$
d F_{\text {revers }}=-S d T-\mathcal{M} d H-H^{*} d \mathcal{M}_{e}+V d q .
$$

For small deviations of the magnetic moment out of equilibrium in zero magnetic field, the above definition can be used, to Taylor expand the free energy in powers of $\tilde{\mathcal{M}}$ :

$$
F(T, H, \tilde{\mathcal{M}}, q, V)=F_{0}\left(T, q, V, \mathcal{M}_{0}\right)+a \tilde{\mathcal{M}}+\frac{b}{2} \tilde{\mathcal{M}}^{2}+\ldots
$$

To determine the parameters $a$ and $b$, which can depend on $T$ and $H$, the equilibrium for fixed $T, q$, and $V$, at zero applied field, is given by:

$$
\frac{\partial F}{\partial \tilde{\mathcal{M}}}=a+b \tilde{\mathcal{M}}_{e q}=0 \Rightarrow \tilde{\mathcal{M}}_{e q}=\frac{-a}{b}=\chi_{S} H
$$

where $\chi_{S}$ is the isothermal, material specific magnetic susceptibility. From the definition of the internal magnetic energy, equation 2.19, and the second term in equation 2.22, it can be seen that $a$ is linear in $H$, giving $a=a^{\prime}-H$. Given that at $H=0$ the equilibrium deviation is also zero, $\tilde{\mathcal{M}}_{e q}=0$ demands that $a^{\prime}=0$. The third term in the Taylor expansion (equation 2.22) states that $b=1 / \chi_{S}$. This leads to the description of the free energy at and near equilibrium:

$$
\left.F(T, H, \tilde{\mathcal{M}}, q, V)=F_{0}\left(T, q, V, \mathcal{M}_{0}\right)\right)-H \tilde{\mathcal{M}}+\frac{\tilde{\mathcal{M}}^{2}}{2 \chi_{S}}
$$

The change of the free energy $F$ can be generated by changing $T, \tilde{\mathcal{M}}$ or $H$ :

$$
\left.d F=\frac{F_{0}}{d T}\left(T, q, V, \mathcal{M}_{0}\right)\right) d T-\tilde{\mathcal{M}} d H+\left(\frac{\tilde{\mathcal{M}}}{\chi_{S}}-H\right) d \tilde{\mathcal{M}}
$$

Now, the magnetization potential $H^{*}$ can be determined by comparing the change of the free energy $d F$ in equation 2.23 with the reversible case in equation 2.21 . For this purpose, the changes in equation 2.23 need to be reversible. This is possible, because the amount of magnetic moment transported through the interface from the ferromagnet into the paramagnetic metal in the presence of nonequilibrium magnetization $\left(\mathcal{M}_{0}\right.$ (Ferromagnet $\left.) \neq 0\right)$ is of relevance. Therefore, the relaxation process can be excluded, assuming $\tau_{D}=\infty$ and $d \mathcal{M}_{i}=0$. The change of the 
magnetic moment is then $d \mathcal{M}=d \mathcal{M}_{e}$. Restricting equation 2.23 to reversibility, gives:

$$
d F_{\text {revers }}=\frac{F_{0}}{d T}\left(T, q, V, \mathcal{M}_{0}\right) d T-\tilde{\mathcal{M}} d H+\left(\frac{\tilde{\mathcal{M}}}{\chi_{S}}-H\right) d \tilde{\mathcal{M}}_{e}
$$

The final examination of the reversible change of the magnetic moment under the conditions of fixed $T$ and $H$ gives, by comparing equation 2.24 and 2.20 :

$$
-H^{*}=\left(\frac{\tilde{\mathcal{M}}}{\chi}-H\right) \text {. }
$$

$H^{*}$ describes the response of magnetization to the external field, hence it is called the magnetization potential. It is the affinity that causes a flux of magnetic moments. A last consideration will show the compatibility of $H^{*}$ with irreversible processes, in which $\tau_{D}$ is finite and internal relaxation takes place. Looking at equations 2.20 and 2.23 under the condition of fixed $T, H, V$, and $q$, in addition to thermal isolation and no magnetization transport, $d Q=d M_{e}=0$ leaves:

$$
-T d S=\left(\frac{\tilde{\mathcal{M}}}{\chi}-H\right) d \tilde{\mathcal{M}}_{i} \Rightarrow d S=-\frac{1}{T}\left(\frac{\tilde{\mathcal{M}}}{\chi}-H\right) d \tilde{\mathcal{M}}_{i}
$$

This shows the change in entropy as the magnetization relaxes into equilibrium. Assuming $d \mathcal{M}_{i} / d t=-(\tilde{\mathcal{M}}-\chi H) / \tau_{D}$, makes the entropy production $\dot{S}$ positive, in agreement with irreversible processes.

These considerations for the exchange of magnetic moments between two discrete systems can be transformed into a transport of magnetization in continuous systems by converting the extensive parameters into intensive parameters through division by the volume density. Then the magnetic moments $\mathcal{M}$ (extensive) are replaced by the magnetization $M$ (intensive), and the flux of magnetic moment $I_{M}$ is replaced by the current density of magnetization $\boldsymbol{J}_{M}$. The continuous system has to be modeled by a sequence of interfaces, as those described in the discrete case, in which the irreversible processes occur. Therefore the rate of entropy production in a thermomagneto-electric system, in which heat, charge and magnetization can flow, is given by:

$$
\dot{s}=\nabla \frac{1}{T} \cdot \boldsymbol{J}_{Q}-\frac{1}{T} \nabla\left(-H^{*}\right) \cdot \boldsymbol{J}_{M}-\frac{1}{T} \nabla \mu \cdot \boldsymbol{J}_{N} .
$$

This is the same equation as equation 2.10, extended by the magnetization potential, being the affinity for the flow of magnetization. $\boldsymbol{J}_{M}$ consists of the fluxes $J_{M_{x}}, J_{M_{y}}$, and $J_{M_{z}}$. Considering the one dimensional case, the direction indices can be dropped, leaving the flux $J_{M}$, which is related to the flux of electrons by:

$$
J_{M}=P m J_{N}
$$

Here, the electric current can be distinguished by spin up and spin down states, $e J_{N}=e\left(J_{N_{\uparrow}}+J_{N_{\downarrow}}\right)$. The parameters are the spin polarization $P=\frac{J_{N_{\uparrow}}-J_{N_{\downarrow}}}{J_{N_{\uparrow}}+J_{N_{\downarrow}}}$ and 


\subsection{Irreversible Thermodynamics and Thermodynamic Transport}

each electrons magnetic moment $m$, given in units of $\mu_{B}$ or $\mathrm{A} \cdot \mathrm{m}^{2}$. The components $J_{N_{\uparrow}}$ and $J_{N_{\downarrow}}$ represent the fluxes of the spin-up and spin-down electrons, respectively. While the dimension of $e J_{N}$ is $\left[\frac{\mathrm{C}}{\mathrm{m}^{2} s}\right]$, the dimension of $J_{M}$ is $\left[\frac{\mathrm{Am}^{2}}{\mathrm{~m}^{2} s}\right]$.

Having obtained all fluxes and generalized forces, finally the extended dynamical equations can be written as:

$$
\begin{aligned}
& -J_{N}=\frac{L_{11}}{T} \nabla \mu+L_{12} \nabla\left(\frac{1}{T}\right)+\frac{L_{13}}{T} \nabla\left(-H^{*}\right) \\
& -J_{Q}=\frac{L_{21}}{T} \nabla \mu+L_{22} \nabla\left(\frac{1}{T}\right)+\frac{L_{23}}{T} \nabla\left(-H^{*}\right) \\
& -J_{M}=\frac{L_{31}}{T} \nabla \mu+L_{32} \nabla\left(\frac{1}{T}\right)+\frac{L_{33}}{T} \nabla\left(-H^{*}\right) .
\end{aligned}
$$

It is immediately notable that if $L_{3 j}=0=L_{i 3}$, the thermoelectric dynamical equations emerge. The first dynamic equation 2.26 extends the first equation 2.11, by a magnetic force, the magnetization potential. This force is responsible for the spin dependent currents in the magnetic tunnel junctions analyzed in the following sections. Hence it is called the thermo-magneto-electric dynamical equation. The Onsager relation $L_{i j}=L_{j i}$ reduces the number of coefficients to six. Four kinetic coefficients can be immediately adopted from the thermoelectric equations:

$$
L_{11}=\frac{\sigma T}{e^{2}}, \quad L_{12}=L_{21}=-\frac{T^{2} \sigma Q_{S}}{e}, \quad L_{22}=T^{3} \sigma Q_{S}^{2}+T^{2} \kappa .
$$

By keeping the temperature $T$ and the magnetic potential $H^{*}$ fixed,

$$
L_{31}=\frac{P m \sigma T}{e^{2}}=L_{13}
$$

can be deduced from the last equation 2.26. The kinetic coefficient $L_{31}$ is not necessary for the description of electric current, but reveals $L_{13}$, which will later determine the magnetic power in the magneto-Seebeck coefficient. For ferromagnetic metals, the spin polarization $P$ is $\sim 0.5-1$. In a perfect tunnel junction the minority channels are closed, such that even a spin polarized current with $P \approx 1$ can be achieved. In paramagnetic metals, the spin polarization is $\approx 0$, and the coupling between the charge and spin current can be neglected. A lack of spin polarization would eliminate the last term in the first equation 2.26, leaving the first thermomagneto-electric dynamic equation in the form as the first thermo-electric dynamic equation.

The remaining coefficients are not associated with the magneto-Seebeck effect, but will be noted briefly to acquire a complete picture. Their deduction can be found in [36]:

$$
L_{33}=\zeta \frac{m^{2}}{e^{2}} \sigma T
$$

where $\zeta$ is a phenomenological parameter. Assuming $\zeta=1$ would give a proper estimate for nonmagnetic metals in the free electron model. However, it would 
underestimate the kinetic coefficient in ferromagnets with $d$-band electron spin polarization and $s$-band electron conductivity. Assuming that $P^{\prime} \neq P$ is the fraction of polarized electrons in the thermoelectric current, the remaining kinetic coefficients are given by:

$$
L_{23}=L_{32}=-\frac{P^{\prime} m}{e} \sigma T^{2} Q_{S}
$$




\subsection{Phenomenological Discussion}

\subsection{Phenomenological Discussion}

The phenomenological point of view is helpful to visualize how the dynamical equations describe the experiment of measuring the Magneto-Seebeck effect, introduced further below.

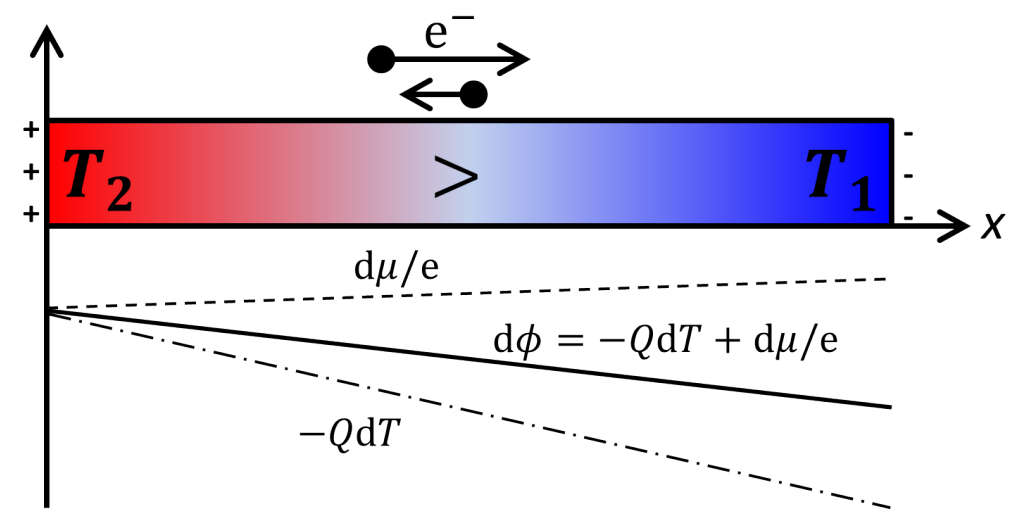

Figure 2.3: A homogeneous metal bar, hot on the left side $\left(T_{2}\right)$, and cold on the right side $\left(T_{1}\right)$. The electrons tend to move towards the lower temperature $T_{1}$. The chemical potential favors a movement towards the higher temperature $T_{2}$, but this effect is much weaker in the free electron model.

\subsubsection{Thermal Conductivity and Thermoelectric Power}

Generally speaking, thermo-electricity is generated by a temperature gradient and thereby a position-dependent velocity distribution of charge carriers (electrons) [2, 50]. Considering a homogeneous conducting material in one dimension along the $x$ axis, in general a metal without an applied external voltage $(j=0)$, where both ends are exposed to different temperatures $T_{1}<T_{2}$, two opposite processes will occur. In the first process, electrons moving away from the region around $T_{2}$ will have a higher velocity due to higher energy, than those moving away from the region around $T_{1}$. Therefore, the net electron movement will be towards the region around $T_{1}$. The number of negative charge carriers in the region with temperature $T_{1}$ will increase and accordingly around the region with temperature $T_{2}$, the number of negative charge carriers will decrease. The second process will work in the other direction, by increasing the chemical potential in the region with the lower temperature and decrease it around the higher temperature, as illustrated in figure 2.3 .

The second process is the affinity of electrons to move towards the lower chemical potential $\mu_{c}$. According to the free electron model, $\mu_{c}$ is lower at higher temperatures, inducing a movement in the opposite direction to that of the thermoelectric power, as equation 2.29, derived in [2, p. 42-47] shows:

$$
\mu_{c} \approx \varepsilon_{F} \cdot\left(1-\frac{\pi^{2}}{12} \frac{k_{B}^{2} T^{2}}{\varepsilon_{F}^{2}}\right)
$$


with $k_{B}$, the Boltzmann constant and the Fermi energy $\varepsilon_{F}$. Starting with the first equation 2.17, the electron current density is given by:

$$
j=\sigma \frac{1}{e} \nabla \mu+\sigma Q_{S} \nabla T .
$$

Since the considered bar is not integrated into a closed circuit loop, there is no current flow $(j=0)$, leading to:

$$
0=\frac{1}{e}\left(\mu_{e}+\mu_{c}\right)+Q_{S} \nabla T .
$$

In a general one dimensional consideration, the total electric field $\mathcal{E}$, which builds up along the metal bar in $\mathrm{x}$-direction, is then given by:

$$
\mathcal{E}=-\frac{1}{e} \nabla_{x} \mu_{e}=Q_{S} \cdot \nabla_{x} T-\frac{1}{e} \cdot \nabla_{x} \mu_{c}
$$

The first term describes the thermo-electric field, induced by the Seebeck coefficient $Q_{S}$. It is the process driving the electrons towards the lower temperature. The second term describes the field, which stems from the difference in the chemical potential, between the two ends of the metal bar. Here it is given a negative sign, because of the definition in equation 2.29 it is working towards the lower chemical potential. This is the opposite direction to that of the thermopower. The total electric field $\mathcal{E}$ can then be expressed by:

$$
\mathcal{E}=Q_{S} \cdot \nabla_{x} T-\frac{1}{e} \cdot \nabla_{x} \mu_{c}=\left(Q_{S}-\frac{1}{e} \cdot \frac{d \mu_{c}}{d T}\right) \cdot \frac{d T}{d x},
$$

with the same Seebeck coefficient $Q_{S}$ as derived from the dynamical equations. It can be calculated using conductance matrices. In the free electron limit it becomes:

$$
Q_{S}=-\frac{\pi^{2}}{2 e} \cdot \frac{k_{B}^{2} T}{\varepsilon_{F}} .
$$

Note that in this definition $Q_{S}$ has a positive value, because the charge of the electron is defined as $e=-1.602 \cdot 10^{-19} \mathrm{C}$. The comparison of both components in the total electric field,

$$
Q_{S}-\frac{1}{e} \cdot \frac{d \mu_{c}}{d T}=-\frac{\pi^{2}}{2 e} \cdot \frac{k_{B}^{2} T}{\varepsilon_{F}}+\frac{\pi^{2}}{6 e} \cdot \frac{k_{B}^{2} T}{\varepsilon_{F}},
$$

shows that the electric field caused by the thermoelectric power is three times larger than the field caused by the chemical potential:

$$
\frac{1}{3}\left(Q_{S} d T\right)=\left(\frac{1}{e} d \mu_{c}\right) .
$$

Therefore, the overall electric potential $d \Phi=-Q_{S} d T+1 / e d \mu_{c}$ is dominated by the thermoelectric power- or Seebeck-term causing a net electric field $\mathcal{E}=-\nabla \Phi$. 


\subsection{Phenomenological Discussion}

\section{Seebeck Effect}

The Seebeck voltage or thermo-diffusion-voltage $V_{S}^{2 \rightarrow 1}$, which is generated by the separation of charge carriers (electrons) between the cold (1) and the warm region (2), is given in the same way, as in the derivation from the dynamical equations:

$$
V_{S}^{2 \rightarrow 1}=\Phi_{2}+\Phi_{1}=\int_{x_{2}}^{x_{1}} \mathcal{E} d x
$$

Inserting equation 2.30 in this integral results in:

$$
V_{S}^{2 \rightarrow 1}=-Q_{S}\left(T_{2}-T_{1}\right)+\frac{1}{e} \cdot\left[\mu_{c}\left(T_{2}\right)-\mu_{c}\left(T_{1}\right)\right]
$$

however, in the phenomenological view it becomes clearer, which role the chemical potential plays, and that it works against the thermoelectric power. Further, for small temperature changes, $\left|T_{2}-T_{1}\right| \ll T_{1}, T_{2}, Q_{S}$ can be considered as not temperature dependent but constant. For metals the Seebeck voltage is of the order of $V_{S} \approx 1-10 \mu \mathrm{V}$ per $1 \mathrm{~K}$ temperature difference. After all, it is difficult to measure this effect directly, because the Seebeck-voltages occurring in contacts and inlet conductors are of the same magnitude. In semiconductors, the Seebeck effect can be utilized to determine the type of doping. If the Seebeck coefficient $Q_{S} \propto 1 / q$ has a negative value, the semiconductor in question is n-type. If $Q_{S}>0$, we are dealing with an p-type semiconductor.

Following figure 2.3, if electrons move, as expected, from the warmer region to the colder, the Seebeck coefficient is positive, and a positive voltage is generated. In the opposite case, a negative Seebeck voltage is generated.

\section{Magneto-Seebeck Effect}

The voltage generated by a temperature gradient along a metal bar can be modified if the bar is divided into two systems (I,II) and reconnected via a junction. This junction can - in addition to the 'ordinary' charge Seebeck effect - constitute a power to move charges by acting as an electron filter or a membrane and allowing only the flow of electrons with certain properties. If the probability of electron transport through the junction is energy independent, the currents in both directions will be equal $j_{I \rightarrow I I}=j_{I I \rightarrow I}$. No net current across the junction will exist, and no additional voltage will be generated. If it is however energy dependent, a net current flow across the junction will generate an additional voltage.

Assuming that the two systems are Ferromagnet I and Ferromagnet II, both systems are connected via a junction that can be a non-magnetic conductor or an insulator, as indicated in figure 2.4 and for simplicity the magnetizations of both systems can either be aligned parallel or antiparallel to each other, then the energy dependence for additional electron transport through the junction is given by the 


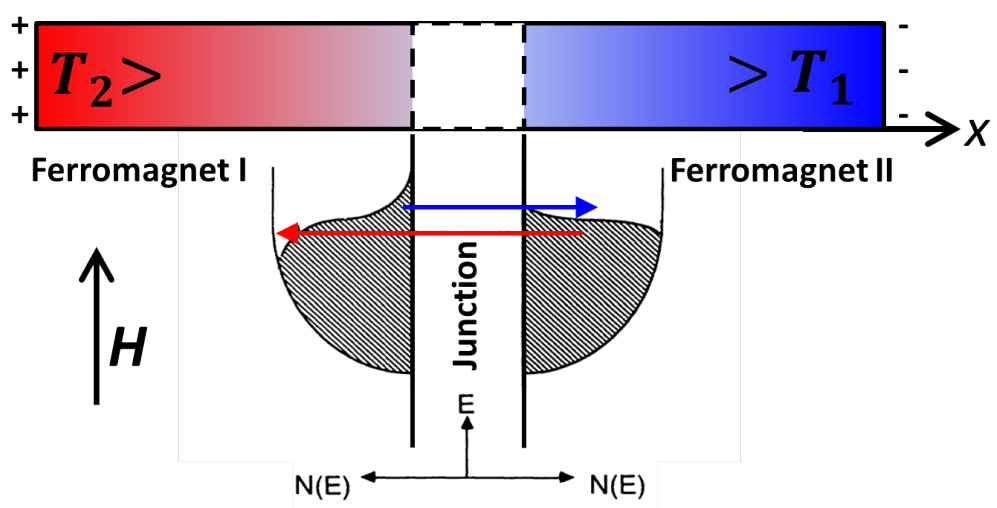

Figure 2.4: A junction connecting two ferromagnetic metals can act as an additional power, to move charges. The number of electrons moved across the junction, can be influenced by the magnetization states of the ferromagnets (see text). The bottom part showing the density of states is taken from [36]. It shows, that electron transport from Ferromagnet I to Ferromagnet II is energetically favored.

relative magnetization of both systems. Going back to the first equation 2.26, the current density in such a system can be described by:

$$
-J_{N}=\frac{L_{11}}{T} \nabla \mu-L_{12} \frac{1}{T^{2}} \nabla T+\frac{L_{13}}{T} \nabla\left(-H^{*}\right) .
$$

In an open circuit as sketched in figure 2.4, $J_{N}=0$. Inserting the results calculated for the kinetic coefficients given in equations 2.27 and 2.28 , leads to:

$$
\begin{aligned}
-J_{N} & =\frac{\sigma}{e} \nabla V-\frac{\sigma Q_{S}}{e} \nabla T+\frac{P m \sigma}{e^{2}} \nabla\left(-H^{*}\right) \Rightarrow \\
0 & =\nabla V-Q_{S} \nabla T-\frac{P m}{e} \nabla\left(-H^{*}\right) \Rightarrow \\
\mathcal{E}=-\nabla V & =-Q_{S} \nabla T-\frac{P m}{e} \nabla\left(-H^{*}\right)=-(Q_{S}+\underbrace{\frac{P m}{e} \frac{\partial H^{*}}{\partial T}}_{Q_{M}}) \nabla T .
\end{aligned}
$$

The net electric field $\mathcal{E}=E+1 / e \nabla \mu_{c}$ already includes the field generated by the chemical potential $\mu_{c}$. Because $1 / e \nabla \mu_{c}$ is weak, compared to the thermo-electric effect, it has no essential influence on the additional contribution from the magnetization. $\nabla V=1 / e \nabla \mu$ is the voltage drop across the junction resulting from the separation of charges, without electric current $\left(J_{N}=0\right) . Q_{S}$ is the pure thermo-electric power, and $Q_{M}$ is the additional thermo-electric power generated by the magnetic properties with the dimension $\frac{V}{K}$. The magnetization potential is $-H^{*}=M / \chi-H$, is derived in equation 2.25. The external field $H$ should not be influenced by the temperature gradient $\partial H / \partial T=0$, therefore, $Q_{M} \sim \partial M / \partial T$. Without a junction, 


\subsection{Phenomenological Discussion}

the electrons would accumulate in the colder region of the system at $T_{1}$, creating the corresponding electric field. With a junction between the two ferromagnets, there are two possibilities, how the electric field and the resulting voltage across the joint system builds up. First, in the parallel magnetization alignment, the majority and minority subbands on both sides of the junction have equal density of states at the Fermi level, therefore the transport of electrons across the junction should not be influenced by the magnetization. In this case also $\partial M / \partial T$ is expected to be small, especially if both ferromagnets have the same saturation magnetization, and the temperature gradient is small. The charge-Seebeck coefficient for the parallel magnetization alignment is given by $S_{P}=Q_{S}+Q_{M_{P}}$. Second, in the antiparallel magnetization alignment, the density of states of the majority and minority subbands on both sides of the junction reverse. That means that there are less free states for majority electrons on the other side of the junction, and consequently the current across the junction is expected be smaller than in the parallel magnetization alignment, leading to a smaller electron accumulation around the area of $T_{1}$ and thus a smaller resulting voltage. In this case, $\partial M / \partial T$ has a discontinuity across the junction, because of the antiparallel magnetization alignment of both ferromagnets. The Seebeck coefficient in the antiparallel alignment is given by $S_{A P}=Q_{S}+Q_{M_{A P}}$.

The difference between $S_{P}$ and $S_{A P}$ depends on the properties of the junction. If the junction is a non-magnetic conductor, separating the two ferromagnets so that their magnetization can be changed separately, the spin polarization $P$ of the electric current across the junction will decrease, leading to a small $S_{P}-S_{A P}$. If the junction is however a tunnel barrier, allowing the transport of fully polarized currents, the difference between $S_{P}$ and $S_{A P}$ is expected to be larger.

However, the difference of both coefficients is not alone decisive to classify the change of the charge-Seebeck coefficients from the parallel to the antiparallel magnetization alignment. Additionally also the magnitude of $S_{P}$ and $S_{A P}$ needs to be taken into account. Therefore, the resulting effect, the magneto-Seebeck effect is expressed through the magneto-Seebeck ratio $S_{M S}$, which is an experimentally accessible quantity expressed by:

$$
S_{M S}=\frac{S_{P}-S_{A P}}{\min \left(\left|S_{P}\right|,\left|S_{A P}\right|\right)}
$$

This phenomenological consideration shows that the magnetization in a junction can influence the electron transport. The magnitude of the magneto-Seebeck effect is expressed through the magneto-Seebeck ratio and can be used to read stored information in the form of parallel and antiparallel magnetization alignment. Later, in section 2.4.3. the currently applied ab-initio method to calculate the magnetoSeebeck and the spin-Seebeck coefficients by using energy dependent transmission functions is introduced. It shows that the voltage or electron accumulation generated by the temperature gradient is more complicated than the phenomenological consideration suggests. The Seebeck coefficients from $a b$-initio calculations are band structure and temperature dependent, and they can have negative values. 


\subsection{Recent Advances in Spincaloritronics}

Spintronics, utilizing in addition to the charge also the spin of electrons for logic and memory devices, is a very vivid research field. Because of the urge for ever smaller devices, ohmic energy dissipation increases threatening with a breakdown of Moore's law. This threat requires the search for new methods, which will avoid this bottleneck and implement a foundation at which the development of smaller and faster devices is assured. One method which gained a lot of recent interest is described by the term 'spincaloritronics', ('calor', Latin for heat). As derived above in the theoretical sections, spin caloritronics deals with charge, spin and entropy transport in devices sized down to the nano-scale. The interest in this field decreased in the 1990s, when the discovery of the GMR effect led to rapidly increasing bit densities on hard discs, and drew the research interest to this direction. In 2006, Gravier et al. [28] picked up this topic in search for thermal effects and published their work on magnetic nanowires. Since then, the interest in this field is rapidly growing. In the following, the most important recent advances in spin caloritronics research will be shortly outlined to show the context of the research presented in this thesis.

\subsubsection{Magneto-Seebeck Effect in GMR Multilayers}

Gravier et al. 28 first measured the magneto-thermo-electric power (MTEP) or magneto-Seebeck effect in multilayered nanowires $(30-60 \mathrm{~nm}$ diameter $)$ consisting of around $300 \mathrm{Cu}$ and Co bilayers, each around $10 \mathrm{~nm}$ thick, etched into polymer membranes. For this purpose, they developed an optical heating method [27]. In this method, a laser diode is used to heat the wires perpendicular to the layer stack, creating a temperature gradient along the wire. The generated voltage is measured perpendicular to the layer interfaces. Lock-in technique is applied to measure the voltage. The laser diode is modulated with a square wave form. They measured a magneto-Seebeck coefficient of around $30 \%$. By applying an additional DC current $I_{D C}$ through the wires, the magneto-thermogalvanic voltage (TMGV) was measured. Compared to previous calculations this situation is given, when a thermovoltage is measured, while $J_{N} \neq 0$. The measured TMGV effect was around $50 \%$. Furthermore, this measurement revealed that heat and charge transport through the wire increases the TMGV linearly with $I_{D C}$ for both, the parallel magnetization alignment of all layers, and when the applied field $H=0$. This linearity indicated a process independent of the thermopower. Within the three current model, which describes the majority spin charge current $j_{+}$, the minority spin charge current $j_{-}$ and the entropy current $j_{S}$, they could explain that sending a DC current $I_{D C}$ causes local temperature gradients between the layers due to the Peltier effect. These local temperature gradients add up to the temperature gradient created by the laser heating and thus increase the voltage. 


\subsection{Recent Advances in Spincaloritronics}

\subsubsection{Spin-Seebeck Effect}

Measuring the spin-Seebeck effect, which is a different effect from the magnetoSeebeck effect, requires the understanding of the spin-Hall effect. This effect was rediscovered in 1999 by Hirsch [31], who also suggested experimental techniques and geometries for its confirmation.

A metal situated in an external magnetic field directed in $z$ direction $B_{z}$, with an electric current moving in $x$ direction $j_{x}$ will build up a potential difference and hence a voltage along $y$ direction. This ordinary Hall effect is caused by the Lorentz force. Moving charge carriers are deflected perpendicular to the direction of their motion and perpendicular to the applied field. Usually, the measured Hall effect is larger than the one calculated from the Lorentz force, because there is an additional significant effect present. This additional effect is the anomalous Hall effect. It depends on the magnetization of the material rather than on the external field. In the case of normal metals, it should only occur together with an applied nonzero field. One mechanism causing the anomalous Hall effect is skew-scattering by impurities or phonons: If electrons are scattered by a central potential, there is an asymmetry, because the orbital and the spin angular momentum are conserved separately. Not only the scattering angle plays a role, but also the relative sign of both momenta. That means, spin up electrons are more likely to scatter along the positive direction of the orbital momentum and the spin down electrons in the opposite direction. In a material with a nonzero magnetization one direction would accumulate more electrons than the other. The second effect playing a role in the anomalous Hall effect is the side jump effect, which describes an anomalous velocity of scattered electrons, leading to a different deflection than the one of unscattered itinerant electrons. The existence of the mechanisms causing the anomalous Hall effect leads to the consideration, what happens in a material with a slab geometry, which is not magnetic and is not exposed to an external field? Because of zero net magnetization, there is an equal number of spin up and spin down electrons, the same number of electrons will be scattered in each direction perpendicular to the direction of the electron flow. Therefore, no charge imbalance will occur on either side of the slab, but since spin-up and spin-down electrons will be scattered in the opposite direction, on each side of the slab, electrons of the opposite spin will accumulate. This accumulation of the same spins on each side of the slab is called the spin-Hall effect.

Uchida et al. used the inverse spin-Hall effect to show the spin-Seebeck effect in permalloy in 2008 [60]. They chose permalloy as a ferromagnetic material in slab geometry, i.e. a $20 \mathrm{~nm}$ thin film of $6 \mathrm{~mm}$ length and $100 \mu \mathrm{m}$ width. Applying a magnetic field of a larger magnitude than the coercive field of permalloy $|H|>H_{C}$, and a temperature gradient parallel to the slab's long side induces a spin current $j_{s}=j_{\uparrow}-j_{\downarrow}$. It needs to be stressed that a spin current does not depend on the flow of charge carriers. The temperature gradient changes the electrical and chemical potential along the length of the permalloy slab. The applied field makes sure that the chemical potential gradients of the spin-up electrons $\nabla \mu_{c, \uparrow}$ and spin-down 
electrons $\nabla \mu_{c, \downarrow}$ have different signs along this direction. In the middle, the chemical potentials of both spins have the same value. This results in a high density of spinup electrons on one end of the slab, decreasing towards the other end, and a high density of spin-down electrons on the other end of the slab, also decreasing towards the first end. That means, one end of the slab is spin-up polarized, and the other end is spin-down polarized. Both spin polarizations decrease towards the middle of the slab, resulting there in zero spin polarization. This difference of spin accumulations along the slab is called spin voltage. To detect this spin voltage, Pt wire layers are placed along the width of the permalloy film, perpendicular to the applied field and temperature gradient. Their thickness is small enough to be considered as having a constant temperature and a constant spin accumulation. Through the contact to the permalloy layer, a spin voltage is generated in the Pt wires by polarizing the electrons around the contact to the permalloy and leaving the other spin sort on the side opposite to the contact. This separation of spins in the Pt wire induces an electromotive force perpendicular to the separation direction. The spin sort accumulated at the Pt permalloy contact will determine the direction of the charge voltage generated in the $\mathrm{Pt}$ wire. That means that at both ends of the slab the voltages along the $\mathrm{Pt}$ wires should have opposite signs. In the middle, the voltage should be zero. The experimental results presented in [60] match these expectations, proving the existence of the spin-Seebeck effect. The experimentally determined spin-Seebeck coefficient for permalloy is $S_{S}=-2 \mathrm{nVK}^{-1}$ at room temperature.

In 2010 Uchida et al. 61] showed the spin-Seebeck effect in isolating YIG ferromagnets. In the same issue of nature materials, Jaworski et al. [33] showed the spin-Seebeck effect in ferromagnetic semiconductors (GaMnAs). Both groups used similar experimental setups, taking advantage of the inverse spin-Hall effect. By using an insulator and by cutting the slab into two parts, the latter in the case of the semiconductor, and still observing a spin accumulation, it was proven that the spin-Seebeck effect does not originate from the diffusion of charges.

At this point, the difference between these two effects, the magneto-Seebeck and the spin-Seebeck effect, becomes clear. The magneto-Seebeck effect is a thermocharge effect additionally influenced by a magnetic force to move charges. While the spin-Seebeck effect is a pure spin current effect without charge transport. The experiment conducted to prove the spin-Seebeck effect using the inverse spin-Hall effect can be explained by spin pumping at the interface between the ferromagnet and the paramagnetic metal. This leads to the inverse spin-Hall effect from which the spin-Seebeck effect can be determined. The magneto-Seebeck effect can be measured directly by measuring the change in voltage with changing magnetization.

\subsubsection{Spin-Seebeck and Magneto-Seebeck Effect}

A deeper understanding of the origin of the spin-Seebeck effect and the magnetoSeebeck effect in magnetic tunnel junctions is given in [17] by Czerner et al. In their work, the Seebeck coefficients were calculated ab-initio.

There are two different possibilities to realize the spin-Seebeck effect. The first one 


\subsection{Recent Advances in Spincaloritronics}
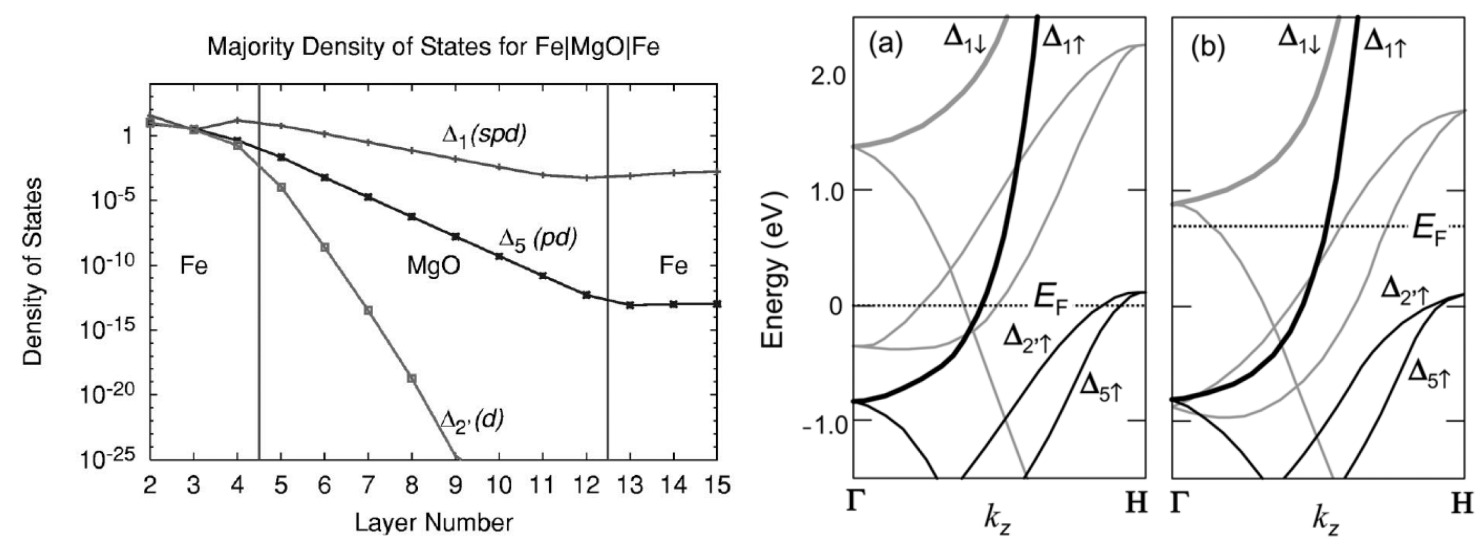

(a) Tunneling density of states in a $\mathrm{Fe}(001) /$ (b) Band dispersion of bcc-Fe (left) and bcc-Co $\mathrm{MgO}(001) / \mathrm{Fe}(001)$ tunnel junction with paral- (right) in the $(001)(\Gamma-H)$ direction, taken from lel magnetization alignment, taken from [12].

[67. The black lines represent the majority spin bands, the gray lines the minority spin bands.

Figure 2.5: Tunneling density of states through a $\mathrm{MgO}$ tunnel barrier (a) and band structure around the Fermi level in bcc-Fe and bcc-Co (b).

is, as experimentally measured, indirectly via the inverse-spin-Hall effect [33, 60, 61]. The spin-Seebeck effect results from different chemical potentials for each spin, given by: $S_{S}=\frac{\mu_{\uparrow}-\mu_{\downarrow}}{\Delta T}$. In the case of one single ferromagnetic material exposed to a temperature gradient and a non-equilibrium magnetization, this effect can be visualized in analogy to a thermo-couple exhibiting the charge-Seebeck effect. However, the two different chemical potentials and the resulting different Seebeck coefficients are in a single material. This leads to varying spin densities throughout the sample along the temperature gradient.

The other possibility originates from different asymmetries in the density of states around the Fermi level for both spin channels. This leads to two spin-dependent Seebeck coefficients, one for each spin. Both spin channels act as a thermocouple, having the spin-Seebeck coefficient $S_{S}=S_{\uparrow}-S_{\downarrow}$. In contrast to the charge-Seebeck effect, where electrons have to be moved in order to separate charges, in the spinSeebeck case both spins are being unequally occupied staying at the same position. That means that spin relaxation processes are expected to break the effect down, e.g. in samples larger than the spin diffusion lengths. Consequently, half-metals are promising candidates for a large spin-Seebeck effect, because of their metallic behavior only for one spin subband and therefore a large spin diffusion length. Nevertheless, this effect was measured experimentally, even in sample sizes larger than the spin diffusion lengths [60].

A different way to realize a large spin asymmetry within the spin channels is by separating two ferromagnetic layers using a junction. This possibility was introduced above in section 2.3.1 from the phenomenological point of view. MgO-based tunnel junctions with $\mathrm{Co}, \mathrm{Fe}$ or $\mathrm{CoFe}$ as magnetic layers, show a large tunnel magne- 
toresistance TMR, because the tunneling currents are highly spin-polarized. Figure 2.5(a) shows the density of states of electrons, tunneling from one Fe electrode to another through an 8 monolayer thick $\mathrm{MgO}$ tunnel barrier for different bands in the parallel magnetization alignment. The density of states for the $\Delta_{1}$ band is much higher than for the $\Delta_{5}$ and $\Delta_{2^{\prime}}$ bands $s^{3}$. Electrons from all bands are contributing to the tunneling current, resulting in a small resistance. In the antiparallel magnetization alignment, the $\Delta_{1}$ states are blocked at the second Fe electrode, and are not contributing to the tunneling current. The much smaller density of states of the $\Delta_{2^{\prime}}$ and $\Delta_{5}$ band electrons contributing to the tunneling current leads to a large resistance and a high TMR. Figure 2.5(b) left shows the electronic band structure of bcc-Fe. At the Fermi level, there are majority $\Delta_{1}, \Delta_{2^{\prime}}$, and $\Delta_{5}$ bands and minority $\Delta_{2}, \Delta_{2^{\prime}}$ and $\Delta_{5}$ bands. That means that the $\Delta_{1}$ band at the Fermi level is $100 \%$ spin polarized. This combination of the Fe band structure and the tunneling probabilities for the $\Delta_{1}$ band electrons through crystalline $\mathrm{MgO}$ let such tunnel junctions act as magnetization dependent spin filters with half-metallic behavior. Figure 2.5(b) right shows the band structure of bcc-Co. At the Fermi level there are the majority $\Delta_{1}$ band, the minority $\Delta_{2}$ and $\Delta_{2^{\prime}}$ bands and the majority and minority $\Delta_{5}$ bands. The Fermi level is energetically higher than in bcc-Fe, due to an additional 3d electron. This leaves the majority $\Delta_{2^{\prime}}$ and $\Delta_{5}$ states below the Fermi level, making bcc-Co the perfect material for $\mathrm{MgO}$-based tunnel junctions. Unfortunately, it is not possible to grow pure Co in bcc structure. That is why for high TMR tunnel junctions combinations of $\mathrm{Fe}$ and $\mathrm{Co}$ are chosen. The magnetization dependent tunneling probabilities through the tunnel junction lead to magnetization dependent Seebeck coefficients for the majority and minority spin electrons $S_{\uparrow}, S_{\downarrow}$. The spin diffusion lengths in Co and Fe are rather small, making it difficult to detect the spin-Seebeck effect in tunnel junctions consisting of a couple of monolayers.

Experimentally easier accessible is the magneto-Seebeck effect. There, the accumulation of charges on each side of the barrier and thus the charge-Seebeck coefficient depends on the relative magnetization orientation of both ferromagnetic layers. The magneto-Seebeck ratio for an angle $\theta$ with respect to $0^{\circ}$ is given by:

$$
S_{M S}=\frac{S\left(0^{\circ}\right)-S(\theta)}{\min \left(\left|S\left(0^{\circ}\right)\right|,|S(\theta)|\right)}
$$

In general, $S\left(0^{\circ}\right)=S_{P}$ is the charge-Seebeck coefficient, when both layer's magnetizations are oriented parallel to each other. Experimentally, it is challenging to measure the angle-resolved Seebeck voltage for defined magnetization alignments between parallel magnetization $\left(\theta=0^{\circ}\right)$ and antiparallel magnetization $\left(\theta=180^{\circ}\right)$ when using pseudo spin-valves, because there is only a small range of the applied magnetic field at which the magnetization is antiparallel. A well defined angle can not be determined because the magnetization switches also via domain wall propagation and not solely via magnetization rotation as it is the dominant process for

${ }^{3}$ In this depiction, the $\Delta_{2}$ band does not contribute to the tunneling current, however, for thinner $\mathrm{MgO}$ layers it might have a nonvanishing contribution. 


\subsection{Recent Advances in Spincaloritronics}

smallest devices below a micron in size. Therefore, only the charge-Seebeck coefficients $S_{P}$ and $S\left(180^{\circ}\right)=S_{A P}$, will be considered in the following. In that case, equation 2.34 can be written in the simpler form of equation 2.33 .

The various Seebeck effects in tunnel junctions with Co, Fe or CoFe leads and $\mathrm{MgO}$ barriers were computed in [17] by performing ballistic transport calculations. Spin-diffusion effects were neglected, and using the energy dependent transmission probability $T_{t}(E)$, the moments $L_{n}$ can be calculated:

$$
L_{n}=\frac{2}{h} \int T_{t}(E)(E-\mu)^{n}\left[-\frac{\partial f(E, \mu, T)}{\partial E}\right] d E,
$$

where $f(E, \mu, T)$ is the Fermi occupation function, the only term that is considered $T$ dependent in this calculation. The conductance $G$ and the Seebeck coefficients $S$ are determined by:

$$
\begin{aligned}
& G=e^{2} L_{0}=\frac{2 e^{2}}{h} \int T_{t}(E)\left[-\frac{\partial f(E, \mu, T)}{\partial E}\right] d E, \\
& S=-\frac{1}{e T} \frac{L_{1}}{L_{0}}=-\frac{1}{e T} \frac{\int T_{t}(E)(E-\mu)\left[-\frac{\partial f(E, \mu, T)}{\partial E}\right] d E}{\int T_{t}(E)\left[-\frac{\partial f(E, \mu, T)}{\partial E}\right] d E} .
\end{aligned}
$$

The transmission probability function $T_{t}(E)$ represents an energy dependent conductivity, multiplied by the states available at the Fermi level, and integrated over the energy, it determines the conductance $G$ from $L_{0}$. In the first moment $L_{1}$, the integrand is multiplied by $(E-\mu)$, which describes the additional energy above the Fermi energy originating from the temperature elevation, and smearing out of the Fermi level. This is the energy needed to calculate the Seebeck coefficient $S$. The conductivity derived earlier from the irreversible thermodynamics model is given by $\sigma=e^{2} L_{11} / T$ (see equation 2.12). Both definitions given in equation 2.12 and in equation 2.35 show a deviation of the factor $1 / T$. This deviation stems from differences in the definition of the dynamical equations. The kinetic coefficients $L_{11}$ and $L_{12}$ are defined by the equations 2.11, while the moments $L_{n}$ are taken from the definition given in [56]. Comparing both definitions, yields:

$$
L_{0}=\frac{L_{11}}{T}, \quad L_{1}=\frac{L_{12}}{T} .
$$

Because of the second quotient in the definition of the Seebeck coefficient $Q_{S}=$ $-1 /(\mathrm{Te}) \cdot L_{12} / L_{11}$ (see equation 2.16), in the definition given in equation 2.35, the factor $1 / T$ disappears. In short that means, the kinetic coefficients $L_{11}$ and $L_{12}$ in the dynamical equations, and the moments $L_{n}$ for $n=1,2$ used for the ab-initio calculations represent the same parameters defined from the conductivity $\sigma$ and the conductance $G$ and the Seebeck coefficients $Q_{S}$ and $S$. The moments $L_{n}$ originate from band structure calculations, taking into account the electronic structure of the leads and the barrier to determine the transmission probabilities $T_{t}(E)$. The magnetization dependence is taken into account by the separate treatment of the 

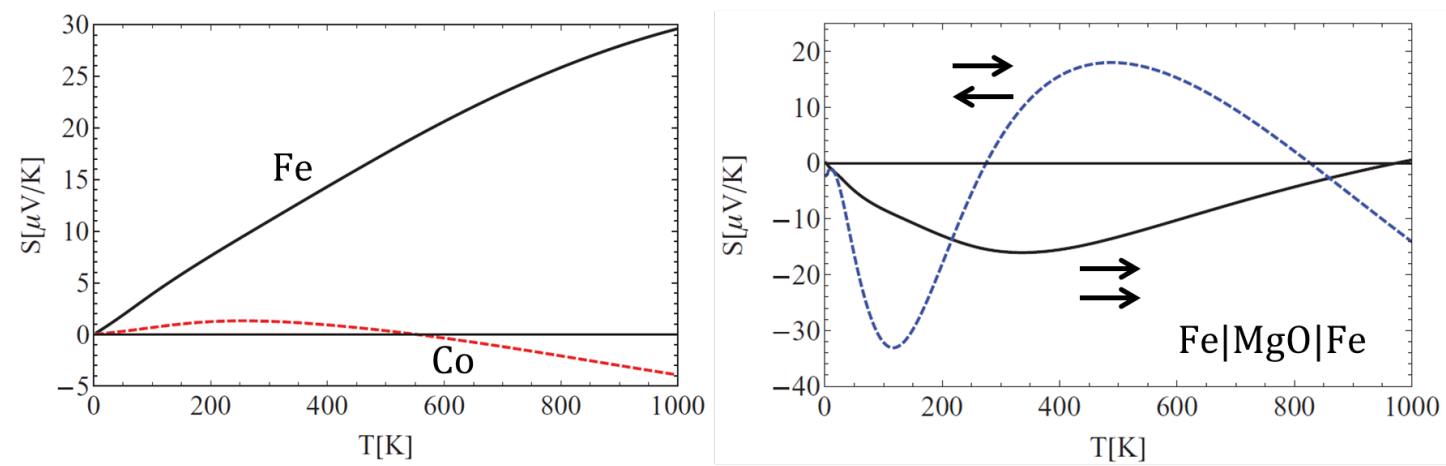

Figure 2.6: Seebeck coefficients for a temperature range from $0 \mathrm{~K}$ to $1000 \mathrm{~K}$. Left: for pure $\mathrm{Fe}$ and $\mathrm{Co}$, and for right: a $\mathrm{Fe}|\mathrm{MgO}| \mathrm{Fe}$ tunnel junction in the parallel and antiparallel magnetization state. Taken from [17.

spin subbands and separately calculating the charge-Seebeck coefficients for each magnetization alignment.

This ab-initio approach enables the calculation of various Seebeck coefficients by the following procedure. First, the spin dependent Seebeck coefficients $S_{\uparrow}$ and $S_{\downarrow}$ are calculated applying spin dependent transmission probabilities $T_{t}^{\uparrow}(E)$ and $T_{t}^{\downarrow}(E)$. The difference of both spin dependent Seebeck coefficients equals the spin-Seebeck coefficient $S_{S}$. Then, the charge-Seebeck coefficient for the entire number of electrons at the Fermi level can be calculated using $T_{t}(E)=T_{t}^{\uparrow}(E)+T_{t}^{\downarrow}(E)$. The transmission probabilities $T_{t}^{\uparrow}$ and $T_{t}^{\downarrow}$ change with the angle between the magnetizations of the two ferromagnetic layers. Therefore, the charge-Seebeck coefficient can be calculated for any angle from parallel to antiparallel, thus giving the magneto Seebeck ratio $S_{M S}$. Both effects show a temperature dependence, which can be explained by having a closer look at the transmission probabilities and the occupation function: The Seebeck coefficient is the geometric center of $T_{t}(E) \cdot\left[-\frac{\partial f(E, \mu, T)}{\partial E}\right]$, divided by temperature. Looking at these functions for different temperatures, it can be seen that the geometric center changes with temperature for each spin channel.

The charge-Seebeck coefficients calculated using this method for pure bcc-Fe and pure bcc-Co already show characteristics that can not be deducted from the thermodynamic transport or the phenomenological considerations, which consider constant charge-Seebeck coefficients - valid only for small temperature gradients and ranges. The charge-Seebeck coefficient of Fe on one hand increases almost linearly with the temperature. This so far is consistent with the phenomenological model for free electrons given in equation 2.31. The charge-Seebeck coefficient of Co on the other hand changes sign around $550 \mathrm{~K}$, as the left graph in figure 2.6 shows. A similar behavior is calculated for a $\mathrm{Fe}|\mathrm{MgO}| \mathrm{Fe}$ tunnel junction. If the magnetizations of both Fe layers are aligned parallel, the Seebeck coefficient stays negative over the temperature range from $0 \mathrm{~K}-1000 \mathrm{~K}$ (see figure 2.6 right)). However, if the magnetizations of both layers are aligned antiparallel, it changes sign three times over the whole temperature range. These different Seebeck coefficients, changing sign through the 


\subsection{Recent Advances in Spincaloritronics}
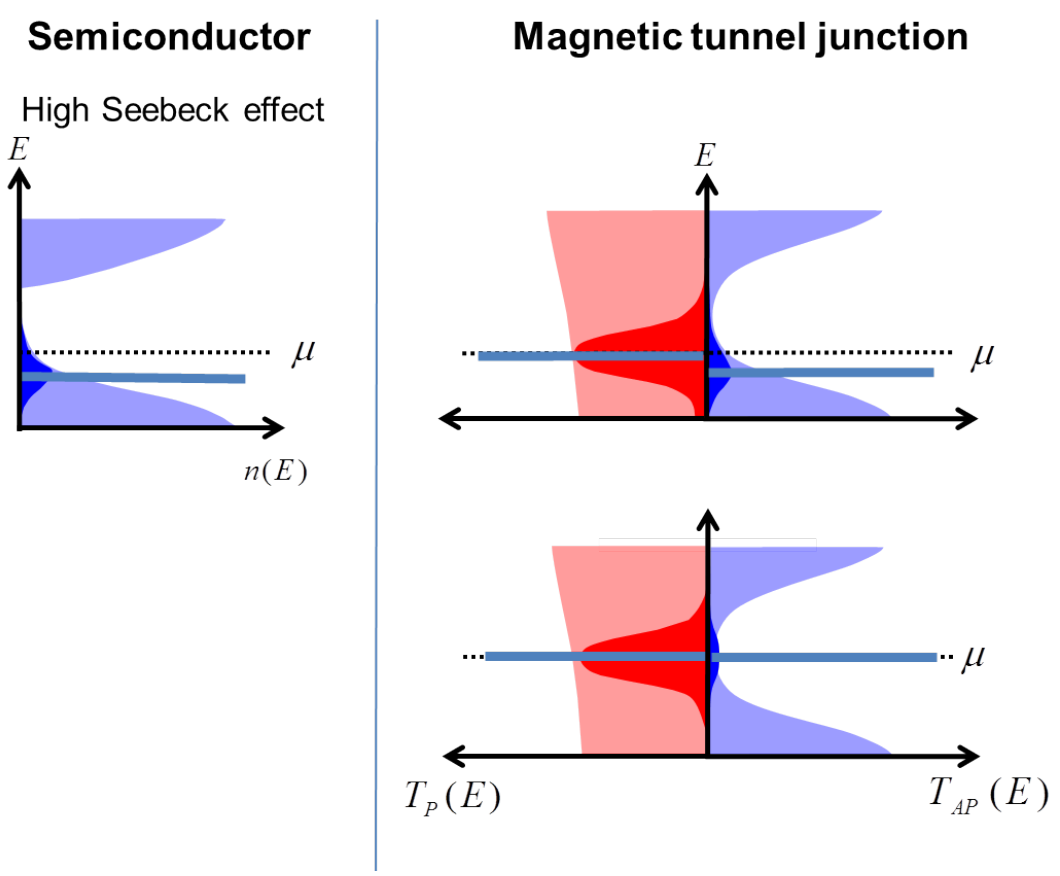

Figure 2.7: The transmission $T_{t}(E)=T_{t}^{\uparrow}(E)+T_{t}^{\downarrow}(E)$ is shown in pale colors. The product of the transmission and $\partial / \partial E \cdot f(E, \mu, T)$ is shown in intense colors. The products geometrical centers are drawn as thick blue lines. Left: A semiconductor shows a large Seebeck effect. Right: A big difference between $T_{P}$ and $T_{A P}$ yields in a high TMR ratio, but does not guarantee a high magneto-Seebeck effect (bottom). A strong asymmetry in the geometric centers is necessary to a have a high magneto-Seebeck effect (top).

temperature range also result in a temperature dependent magneto-Seebeck ratio $S_{M S}$. These differences in the Seebeck coefficients for the pure metals stem from their different band structures at the Fermi level. With increasing temperature, the Fermi level smears out and the electron energies increase. Consequently, the occupation function $f(E, \mu, T)$, and its derivative change with $T$. Further, through the change of $\partial / \partial E \cdot f(E, \mu, T)$ with temperature, other transmission functions contribute to the integral and influence the moments $L_{n}$.

It needs to be stressed that both, the spin-Seebeck coefficient $S_{S}$, and the magnetization dependent charge-Seebeck coefficients $S_{P}$ and $S_{A P}$ can be calculated starting out with the spin-dependent transmission probabilities. However, it is not possible to calculate these effects from each other. They are physically different effects and are not related to each other by a mathematical function. This may be different in the case of $\mathrm{Al}_{2} \mathrm{O}_{3}$-junctions where, calculations using Julier's law lead to simplifications [43].

In order to achieve a large magneto-Seebeck ratio, not the asymmetry in the transmission for each spin channel alone is decisive, but also the geometrical centers of the product of the transmission function $T_{t}(E)=T_{t}^{\uparrow}(E)+T_{t}^{\downarrow}(E)$ and $\partial / \partial E \cdot f(E, \mu, T)$ 
need an asymmetry in the energy level for the parallel and antiparallel configuration, because only then the difference between $S_{P}$ and $S_{A P}$ is large, as depicted in figure 2.7. On the left, a semiconductor is always showing a large magneto-Seebeck effect, because the energy gap and the density of states allow for a geometrical center that is always below or above the Fermi level, depending on the doping. On the right, both tunnel junctions show a high TMR, because they have a high spin polarization at the Fermi level. Only the top junction shows in addition to the high TMR a high magneto-Seebeck effect, because there is an asymmetry in the geometric centers of the product of the transmission with the derivative of the Fermi occupation function, indicated by the thick blue lines. If an asymmetry is not present, the transmission probability does not change when shifting the energies of the subbands by changing the layers magnetization alignment.

\subsubsection{Experimental Approaches to the Magneto-Seebeck Effect}

Recently, there have been two experimental approaches to measure the magnetoSeebeck ratio. Both use MgO-based MTJs, but differ by the method of producing heat:

One method is realized by resistive heating with micro heater lines on top of the tunnel junctions, by Liebing et al. [42]. Currents applied through these lines produce heat with the power up to $P_{\text {heat }} \approx 60 \mathrm{~mW}$. Heat is generated on top of the tunnel junction, and the bottom of the junction is kept at lab temperature, creating a temperature gradient across the junction. The temperature gradient achieved by the highest heating power is $\Delta T \approx 50 \mathrm{mK}$. With this method they measure magneto-Seebeck ratios $S_{M S} \approx 40 \%$ for junctions showing TMR ratios around $140 \%$. This approach has the advantage that knowing the resistivity of the heater line material, a current dependent temperature on top of the junction can be calculated. Capping layers between the heater line and the top magnetic layer, complicating the access are not necessary. However, placing the heater lines makes the production process of the junctions more complex, and misplaced heater lines do not generate the necessary temperatures and temperature gradients. Besides that, currents through the heater lines can produce unwanted currents through the junction in the case of short circuits, distorting the voltage measurement through additional heating from the Peltier effect. On the other hand, resistive heating allows development and testing.

The other method, optical heating by a laser beam, will be the subject of this thesis and discussed in the subsequent sections. It has the advantage that there are no currents generated, which might possibly disturb the voltage generated by the temperature gradient, because the heating is done optically. The heat is created on one side of the tunnel junction by depositing the energy of a laser beam, while the other side stays at the initial temperature, thus creating a temperature gradient across the tunnel barrier. The laser spot can be placed to a precision of $1 \mu \mathrm{m}$ on the sample surface, giving the flexibility of utilizing all junctions produced on the sample. The usage of intense laser pulses enables the generation of high 


\subsection{Recent Advances in Spincaloritronics}

temperatures and thus high temperature gradients covering the examination of a large temperature range. The challenge in using this technique lies in the precise determination of the de facto generated temperatures and temperature gradients. Until now, the temperature gradient can not be measured directly, but needs to be accessed indirectly through finite element simulations. 


\subsection{Measurement of the Magneto-Seebeck Effect in TMR Junctions}

\subsubsection{The Experimental Setup}

Heat transport experiments on TMR junctions were performed to compare the theoretically predicted magneto-Seebeck ratios to experimental data. Figure 2.8 shows the structure of the samples (top) and the experimental setup. Laser light is used as a heat source to create a temperature gradient across the TMR junction (bottom). The setup is based on the method to measure thermo electric power TEP introduced in [27].

\section{The Sample Structure}

There are $10 \times 10$ tunnel junctions placed on a $1 \times 1 \mathrm{~cm}^{2} \mathrm{SiO}_{2}$ substrate, see figure 2.8 top left. The Au frame around the junctions forms the access to the bottom electric contacts of all junctions in the sample. The details of the bottom contacts are given below. Each junction is covered by a Au contact pad, which forms the top contact of the junction (top middle). Therefore, the junctions are heated through the Au contact pads. All junctions considered for measurement need to be contacted to a voltmeter. Heating the top layer optically creates a temperature gradient across the junction, generating a voltage between the top and bottom contact. The sizes of the junctions range from $30 \mu \mathrm{m}$ edge length down to $0.5 \mu \mathrm{m}$ edge length (for more detail, see figure C.1 in appendix C). However, the large junctions $-30 \mu \mathrm{m}$ and $12.5 \mu \mathrm{m}-$ usually show a low TMR due to the large area of the tunnel barrier and are therefore usually discredited from the beginning for the thermal measurements.

\section{The Optical Setup Arrangement}

The sample is placed between the pole-shoes of an electromagnet, see figure 2.8 bottom left, where in-plane magnetic fields from $-80 \mathrm{mT}$ to $80 \mathrm{mT}$ can be applied. The laser beam is focused to the size of the tunnel junctions, around $10 \mu \mathrm{m}$ in diameter (FWHM), hitting the sample perpendicular to its plane. The focusing is achieved by a Mitutoyo Plan Apo M objective with a $10 \times$ magnification at a focal length of $20 \mathrm{~mm}$ and a working distance of $33.5 \mathrm{~mm}$. A beam splitter is placed into the optical path between the laser and the focusing objective to direct the reflected light into a CCD camera. This part works as a confocal microscope. It enables the positioning of the laser beam on the right spot of the junction with the help of a picture seen on camera, similar to the depiction in figure 2.8 top middle. Since in this setup the optics are fixed, the sample has to be moved with respect to the laser beam in order to choose the junction to be heated. Creating a temperature gradient across the $\mathrm{MgO}$ tunnel barrier generates a voltage. The variation of the external magnetic field enables the measurement of $U(H)$ curves. The surface temperature 


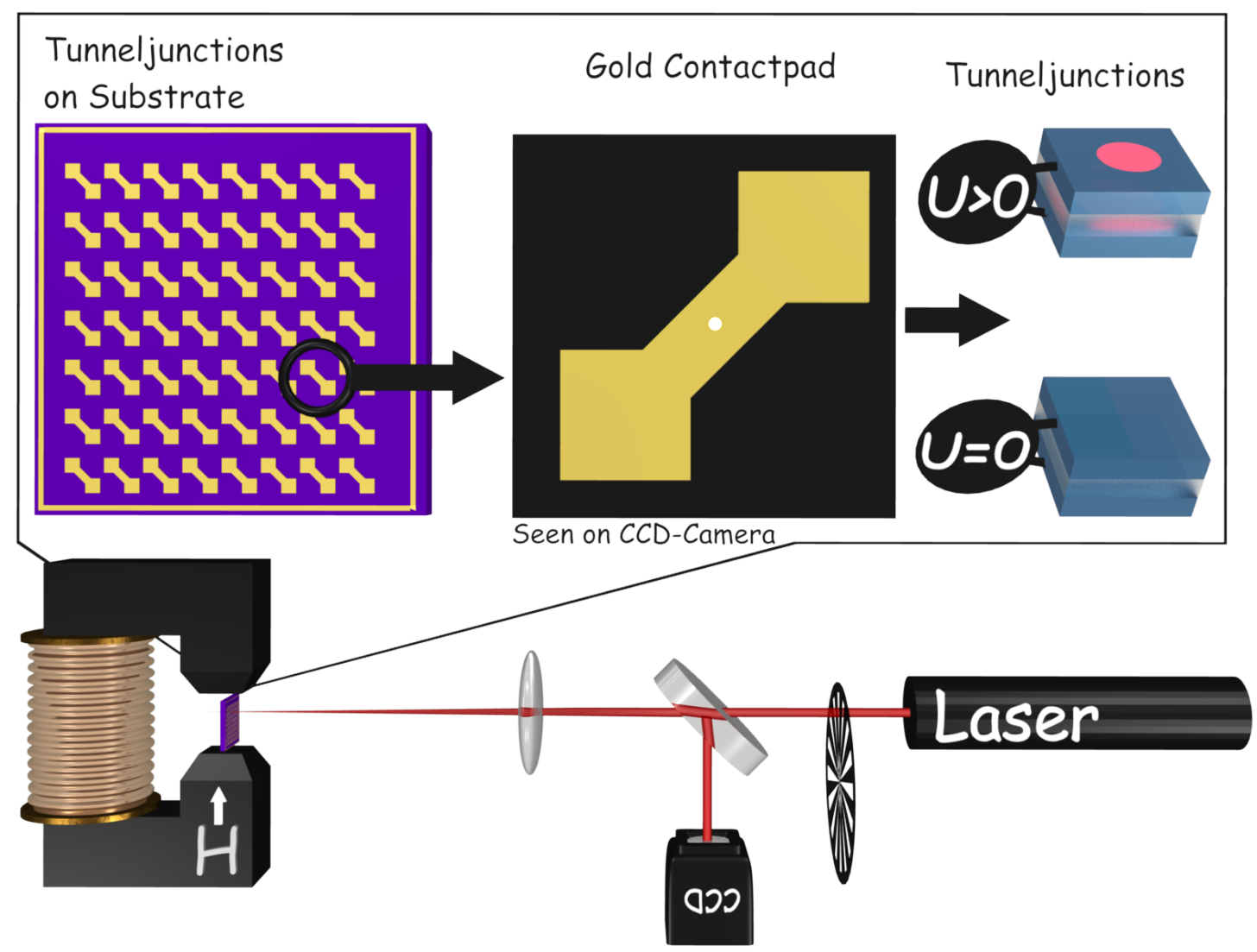

Figure 2.8: Top: Left: The $1 \times 1 \mathrm{~cm}^{2} \mathrm{SiO}_{2}$ substrate contains $10 \times 10$ tunnel junctions. The $\mathrm{Au}$ frame around the junctions forms the bottom contact to the tunnel junctions. Middle: Each junction is covered by a Au contact pad, forming the top contact. Through this Au layer, also the junction underneath is heated. Right: The tunnel junction is heated, building up a temperature gradient across the junction, generating a voltage between the top and bottom contact. Bottom: The sample is situated between the pole-shoes of an electromagnet. The laser beam is focused on the tunnel junction having a diameter of $\sim 10 \mathrm{~nm}$. Positioning of the laser beam is performed utilizing a confocal microscope and a CCD camera. For modulation a mechanical chopper is applied.

is increased with the laser density. Usually, the temperature of the top CoFeB layer and the temperature gradient increase together with the surface temperature.

\section{The Sample Installation}

For the measurement both ferromagnetic layers need electrical contact to build a thermocouple. For this purpose, the sample is installed on a chip carrier, allowing 8 channels. The contacts to the chip carrier are made using a wire bonder. Usually two channels are used to contact the bottom electrodes of all junctions, in case one 
contact is damaged. Through the Au contact pads, up to 6 individual junctions can be contacted to the remaining channels on the chip carrier. The channels at the chip carrier allow direct connections to the measuring electronics through BNC connectors.

\section{TMR Measurements}

Before contacting the junctions and between measurements of the magneto-Seebeck effect, the TMR ratio is monitored. This is done using a Keithley 2400 sourcemeter. It allows to apply a voltage across the junction $(U=10 \mathrm{mV})$ and measure the current through it. Before installing the sample in the charge carrier, this allows to discard broken junctions, usually those that allow currents $I>5 \mu \mathrm{A}$. Besides that, junctions showing a larger TMR effect seem more promising to show a larger magneto-Seebeck ratio. Throughout the thermal measurements, measuring the TMR ratio allows to check, whether the tunnel barrier has been damaged through discharge. The $\mathrm{MgO}$ barriers are very sensitive to discharges, a voltage above $1 \mathrm{~V}$ from the measurement equipment can damage the junction.

\subsubsection{Signal Optimization Process}

\section{The Right Choice of Heating}

Three different lasers were tested throughout the development and the optimization of the setup. First a cw He-Ne laser with an output power of $6 \mathrm{~mW}$ at a wavelength of $633 \mathrm{~nm}$ had been applied, allowing power densities of $\sim 10^{3} \mathrm{~W} / \mathrm{cm}^{2}$ that can be deposited at the sample. That He-Ne laser had not delivered a high enough power density and its power stability had not been sufficient enough. Without variable output power, temperature dependent measurements had been difficult. The need of higher output powers led to the installation of a stabilized semiconductor $\mathrm{cw}$ laser diode with a wavelength $\lambda=784 \mathrm{~nm}$, Toptica DL-100. This laser allowed variable output powers up to $80 \mathrm{~mW}$ (reaching power densities $\sim 10^{4} \mathrm{~W} / \mathrm{cm}^{2}$ ). Additionally to that, also an amplified femtosecond Ti:Sapphire laser was coupled into the experiment. This allowed even higher excitation powers, having energies around $3 \mu \mathrm{J}$ per heating pulse. Furthermore, a pulsed laser made also autocorrelation measurements possible to determine the timescales, at which a temperature gradient is present, after depositing the pulse energy at the sample surface.

Later, a further extension of the setup will allow the performance of reflectivity measurements, like those used in Chapter 1, to determine the surface temperature, reached by the laser heating. This will improve the capability of simulated temperature distributions along the junction. 


\section{Optimizing the Modulation}

The voltage generated by the temperature gradient across the tunnel barrier is measured using a lock-in technique. This technique requires a modulation of the signal, which is achieved by a mechanical chopper. Starting with a chopper blade that allows a frequency of $0.8 \mathrm{kHz}$, i.e. $0.625 \mathrm{~ms}$ for heating and the same amount of time for relaxation. These long heating and cooling periods lead to a noisy signal, in which the magneto-Seebeck ratio is hardly distinguishable. Different chopper blades with higher slot densities allow higher frequencies up to $3 \mathrm{kHz}$ and improve the signal to noise ratio in the measured signal. The final improvement was achieved by installing an 'asymmetric' chopper blade, illustrated in figure 2.9. That means, the open sections in the blade are with one fourth of the period smaller than the closed ones. This results in a heating time of $0.1 \overline{6} \mathrm{~ms}$, and a relaxation time of $0.5 \mathrm{~ms}$ at a frequency of $1.5 \mathrm{kHz}$. At $3 \mathrm{kHz}$ both times halve.

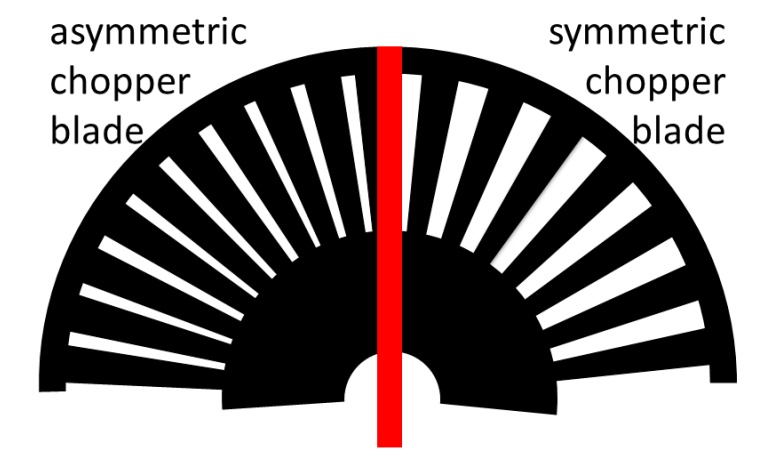

Figure 2.9: Parts of two different chopper blades. On the left, one that has smaller open slots than closed slots, called 'asymmetric' chopper blade. On the right, one with equally sized slots, called 'symmetric' chopper blade.

\section{Improving the Electronics}

In [27] it was found out that modulation frequencies of the heat source above $32 \mathrm{~Hz}$ cause fluctuating currents in the thermocouple, leading to an overestimation of the charge-Seebeck coefficient. Beyond that, in [28] it was observed that additional Peltier heating caused by a DC current occurs and increases the Seebeck effect, because it influences the temperature gradients between the layers.

To minimize the possible current flow through the junction and avoid those effects, an impedance converter $I C$ is connected in series. The LT1113 operational amplifier $O A$ from Linear Technology with a band width of $5 \mathrm{MHz}$, is connected as a voltage follower and increases the lock-in input impedance from $10 \mathrm{M} \Omega$ to $100 \mathrm{G} \Omega$. The top contact of the tunnel junction is connected to the non-inverting input of the $O A$. The output of the $O A$ is connected to the inner core conductor of a coaxial cable. The bottom electrode is directly connected to the outer shield conductor, 
enabling the use of a single input at the lock-in amplifier. To avoid the effect of cable capacities, the impedance converter is installed as close as possible to the sample, directly to the chip carrier channel outputs. The usage of batteries instead of a power adapter assures the elimination of line related noise.

\subsubsection{The Examined Samples}

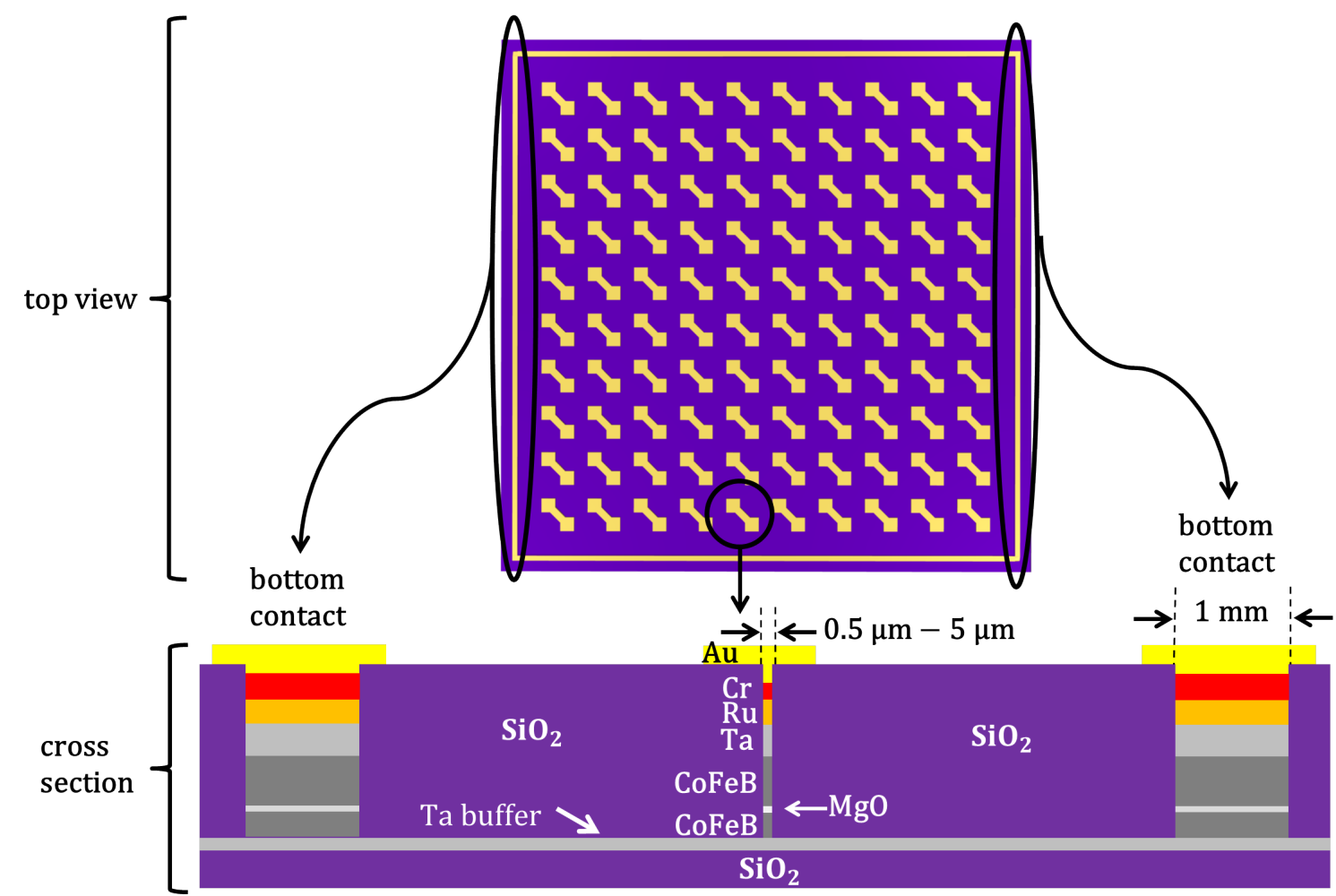

Figure 2.10: Top view and cross section of the examined samples' structure, not to scale. In the middle, a single junction is sketched schematically for illustration $(0.5 \mu \mathrm{m}-5 \mu \mathrm{m}$ edge length). The ohmic contact to the bottom $\mathrm{CoFeB}$ layer is established through the Ta buffer layer and the large area of the wide $(\sim 1 \mathrm{~mm})$ Au capped frame.

All tunnel junctions examined within this thesis are $\mathrm{CoFeB}|\mathrm{MgO}| \mathrm{CoFeB}$ junctions. The detailed description of the fabrication procedure and used equipment is given in [68, Chapter 2]. The cross section of the samples' structure is sketched in figure 2.10 (not to scale). The middle stack represents a tunnel junction: It consists of two CoFeB layers separated by a $2.1 \mathrm{~nm}$ thick $\mathrm{MgO}$ tunnel barrier. According to TEM imaging, this thickness corresponds to ten monolayers [23]. Both CoFeB layers have different thicknesses and thus different coercive fields. This arrangement in the layer stack composes a pseudo spin-valve. The difference to a spin-valve is that a pseudo spin-valve does not contain an anti-ferromagnetic layer adjacent to 


\subsection{Measurement of the Magneto-Seebeck Effect in TMR Junctions}

one of the $\mathrm{CoFeB}$ layers, pinning its magnetization to one direction through exchange bias. Then, only the free (not-pinned) layer's magnetization can change its direction in the applied external field, enabling an extended region for the antiparallel magnetization alignment of both layers. In a pseudo spin-valve, the parallel and antiparallel magnetization alignment strongly depend on the difference between the coercive fields of both layers, leaving only a small region for the antiparallel magnetization alignment. However, less magnetic layers minimize the occurrence of additional overlay effects.

For measurement, a junction needs to be contacted with an electric circuit. This is enabled by two contacts. First, the top contact is established by a $\sim 100 \mu \mathrm{m}$ wide dumbbell-shaped Au pad layer on top of the junction (see figure 2.10 top view). Second, the bottom contact is established through the bottom Ta layer, which at the same time serves as an adhesive layer. This Ta layer connects the bottom CoFeB layer to the frame around the junctions. This frame is made up from the same layer stack as the junctions, but has a much larger area ( $\sim 1 \mathrm{~mm}$ wide), which breaks the isolating properties of the $\mathrm{MgO}$ barrier, ensuring an ohmic contact to its $\mathrm{Au}$ top layer. This frame creates the contact to all junctions on the sample, accessible from the top of the sample. The remaining layers between the junction and the $\mathrm{Au}$ contact pad are: First, a Ta layer with a Ru capping to prevent oxidation during the ex-situ annealing and the preparation of the contact pads after the structuring process. Ta is chosen, because it does not interfere with the crystallization process of the $\mathrm{CoFeB}$ layer that is initiated at the interface to the $\mathrm{MgO}$ barrier. Second, a Cr layer which serves as an adhesive layer. If there is no adhesive layer underneath a Au contact pad, it can be detached from the sample during characterization for the TMR effect in the probe station, where metallic needles are pressed onto the pad for electric contact.

The layer thicknesses and annealing parameters of all three examined samples are given in table C.1 in appendix C. The Ta and $\mathrm{CoFeB}$ layers are Magnetron sputtered. A CoFeB sputter target has the designated concentrations $\mathrm{Co}_{20} \mathrm{Fe}_{60} \mathrm{~B}_{20}$, i.e. the Co:Fe ratio is $1: 3$. However, the measured concentration reveals a Co:Fe ratio of $1: 2$. The $\mathrm{MgO}$ and the $\mathrm{Ru}$ layers are e-beam evaporated in an UHV chamber. The $\mathrm{Cr}$ and $\mathrm{Au}$ layers are evaporated in a different $\mathrm{HV}$ chamber, after structuring. The annealing process is performed ex-situ in an especially constructed chamber, where the sample is situated in an in-plane magnetic field $(\leq 500 \mathrm{mT})$. 


\subsection{Experimental Results}

In this section some representative experimental results will be presented in the course of the development of the experimental setup and discussed with regard to theoretical ab-initio calculations using the method discussed in section 2.4.3.

\subsubsection{The Temperature Gradient}

Simulations performed by A. Zeghuzi and M. Walter [69 $]^{4}$ to calculate the temperatures and temperature gradients across $\mathrm{MgO}$ barriers in tunnel junctions, so far provide the most reliable values for these quantities. Until now, the temperatures and their gradients in such micron-scaled elements can not be accessed experimentally. For those simulations, first the grid of the exact layer stack as those used in

\footnotetext{
${ }^{4}$ The simulations were done applying COMSOL Multiphysics simulation software with the heat transfer module.
}

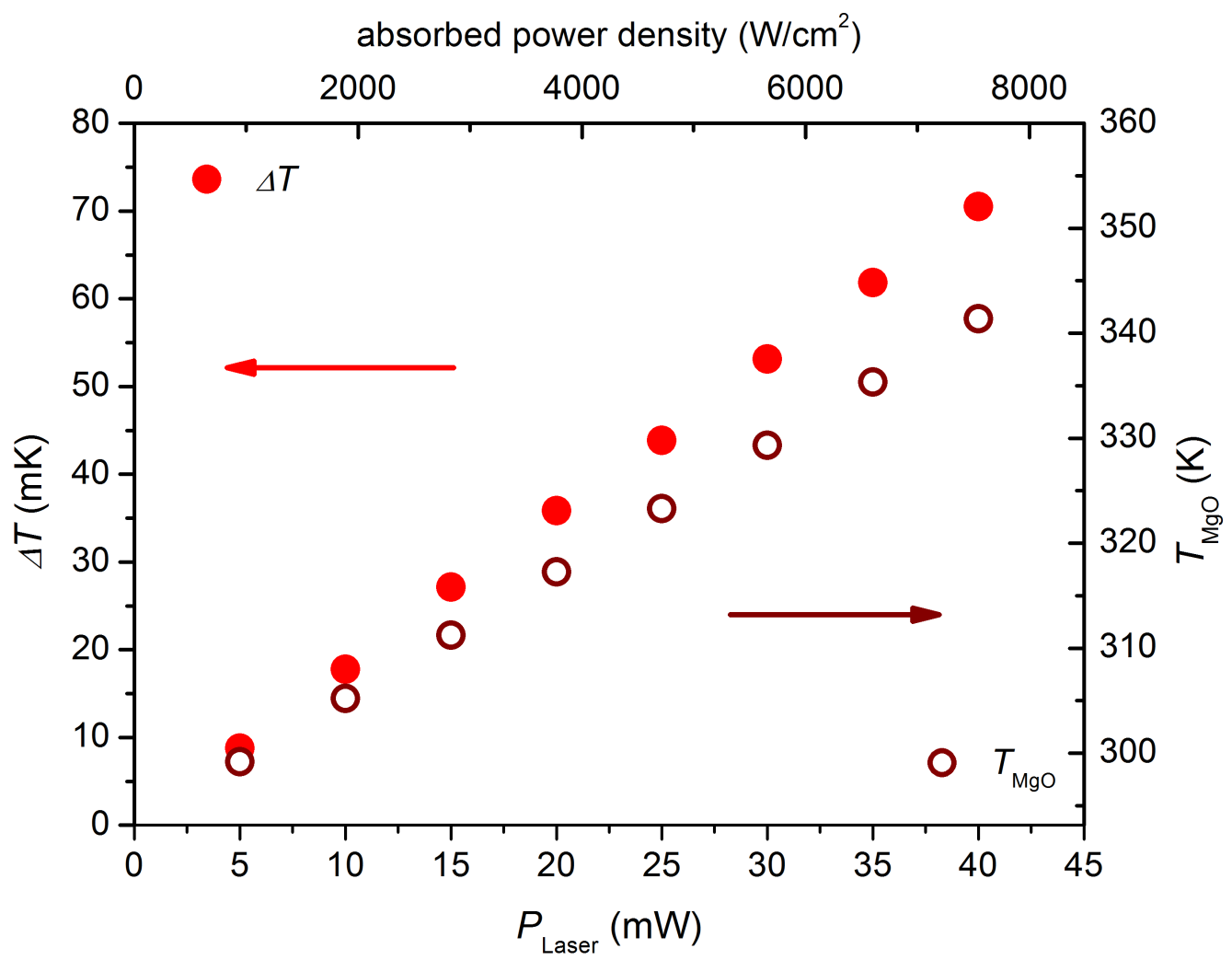

Figure 2.11: The temperature difference $\Delta T$ across the $\mathrm{MgO}$ tunnel barrier, red closed circles, left scale, and the average temperature $T$ of the $\mathrm{MgO}$ tunnel barrier, bordeaux-red open circles, right scale. Both plotted against the laser power deposited at the top of the Au contact pad, and the absorbed power density. Taken from simulations made for [69]. 


\subsection{Experimental Results}

the experiments with a surrounding of $1 \mu \mathrm{m}$ is created. To decrease simulation time and for simpler modeling of the grid using the finite elements method, a cylindric shape of the layer stack with $1 \mu \mathrm{m}$ in diameter is chosen. Besides that, due to limitations of the optical lithography process, at these sizes the junctions are rather circularly than quadratically shaped. The simulation begins with the deposition of a defined laser power density at the surface of the Au contact pad, starting the heating. Then the heat distribution in equilibrium is calculated, considering the thickness, the heat conductance, the density and the heat capacity of each layer in the stack, while the laser power density is deposited constantly. In order to obtain the absorbed power density, intensities from $5 \mathrm{~mW}$ to $30 \mathrm{~mW}$ and a beam diameter of $15 \mu \mathrm{m}$ are assumed, similar to those measured in the experimental setup. The deposited power is divided by three, because roughly one third of the light is absorbed by metals and contributes to heating. The remaining light is reflected or transmitted. The resulting equilibrium temperatures and temperature gradients calculated in the simulations are plotted against $P_{\text {Laser }}$ in figure 2.11. Both quantities, the average temperature and the temperature difference between the top and the bottom of the $\mathrm{MgO}$ layer increase with the applied laser power. Both relations are linear within the range of the examined intensities. The resulting laser power densities are displayed in the top $\mathrm{x}$-axis in figure 2.11.

The final temperature and the temperature difference are both extracted from the heat distribution in equilibrium. This can be done, because these simulations refer to constant heating with a $\mathrm{cw}$ laser. In general for a $\mathrm{cw}$ laser, the temperature begins to increase when heating starts, until it reaches a maximum point at saturation and an equilibrium state at a higher temperature appears. For the considered junctions this equilibrium is reached at around $1 \mu \mathrm{s}$ after the heating has started. In this state also the temperature gradient across the $\mathrm{MgO}$ layer is the largest. Figure 2.12. adapted from [64], shows the temperature distribution in a cross-section through the center of a layer stack at $200 \mathrm{ps}$ (a) and $1 \mu \mathrm{s}$ (b) after heating with $30 \mathrm{~mW}$ has started. While at $200 \mathrm{ps}$ after heating has started the temperature of the area around the $\mathrm{MgO}$ layer has not changed significantly, leaving no range for a temperature gradient, at $1 \mu \mathrm{s}$ after heating has started the temperature around the area of the $\mathrm{MgO}$ layer has increased by over $30 \mathrm{~K}$. The temperature gradient at the same time has also increased to $53 \mathrm{mK}$, as is shown in the temperature profile along the center of the layer stack in figure 2.12(c). Figure 2.12(b) shows additionally that the heat distribution takes place mainly along the metallic components of the junction, while the $\mathrm{SiO}$ surroundings largely remain at room temperature.

Using a pulsed femtosecond laser as a heat source produces a more complicated temporal evolution of the heat distribution than when using a cw laser where the temperature constantly increases towards an equilibrium. According to first simulation results, the intense pulses with energies of $0.2 \mu \mathrm{J} / \mathrm{Pulse} \stackrel{\wedge}{=} 50 \mathrm{~mW}$ at a repetition rate of $250 \mathrm{kHz}$ can generate temperature gradients up to $22 \mathrm{~K}$ across the $\mathrm{MgO}$ layer, decaying within $50 \mathrm{ps}$. The measurement of the voltage is modulated in the $\mathrm{kHz}$ range, i.e. the heating times are in the sub ms region. Therefore, for both heating 


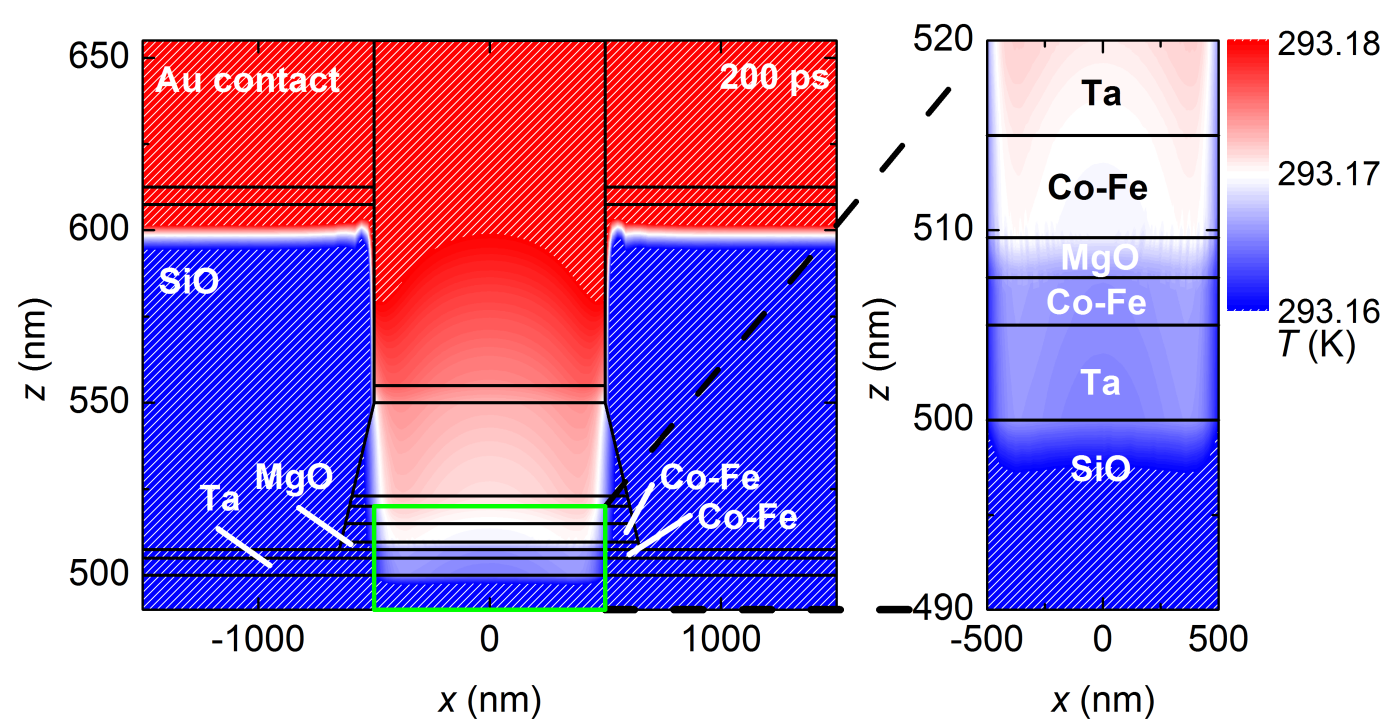

(a) Temperature profile $200 \mathrm{ps}$ after heating has started.

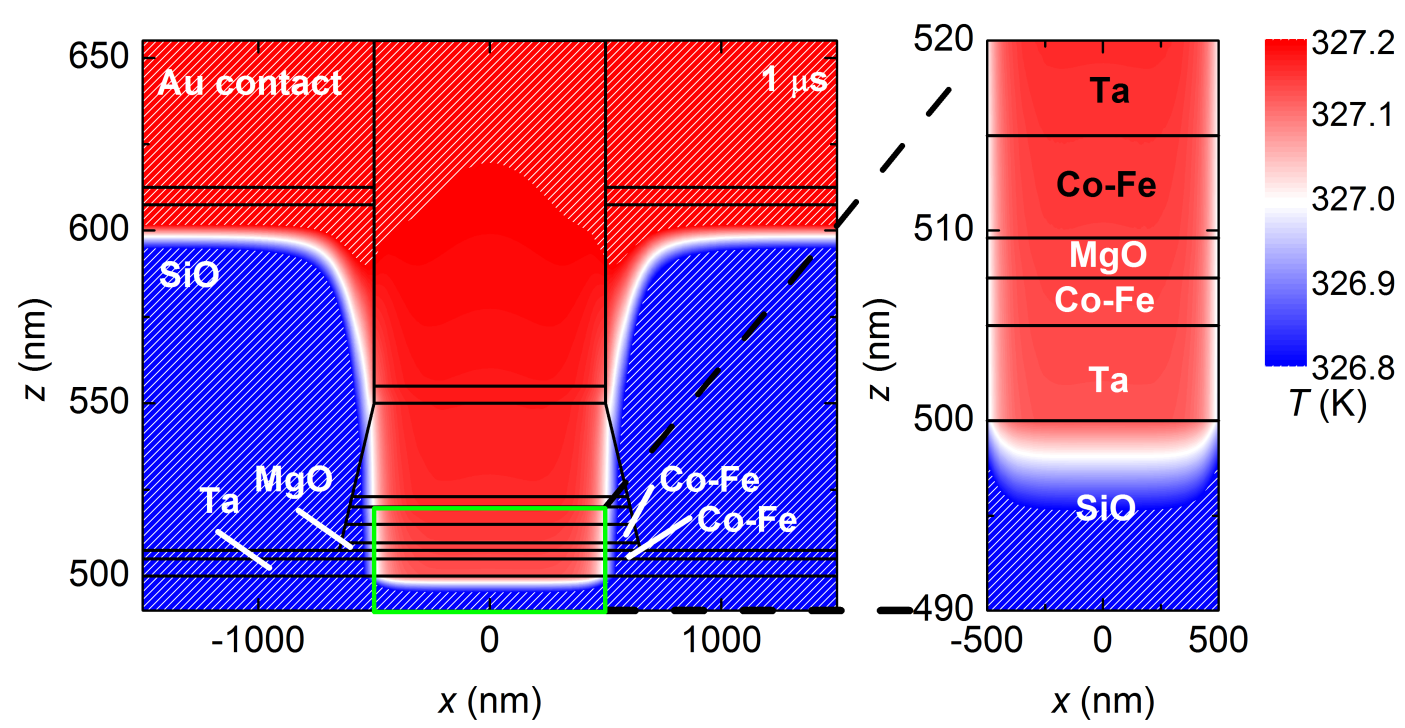

(b) Temperature profile $1 \mu$ s after heating has started.

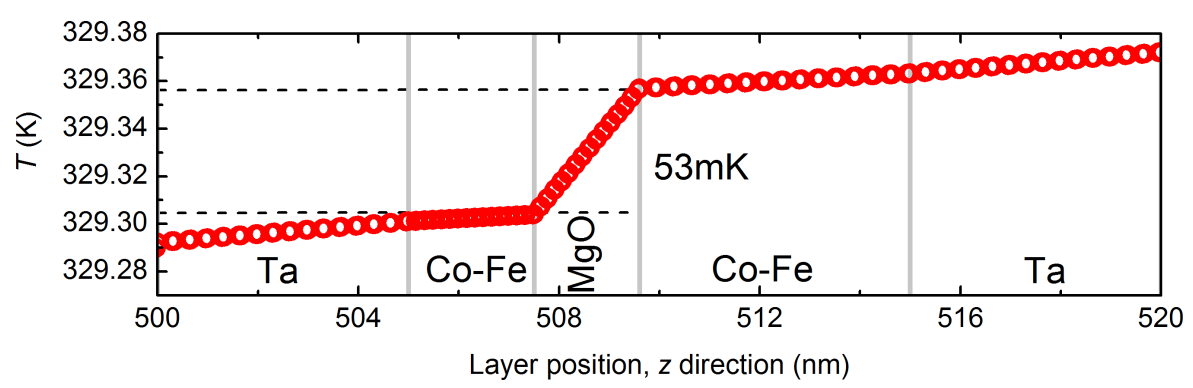

(c) Stationary temperature along the center of the layer stack.

Figure 2.12: Color coded cross-section temperature profile through the center of a MTJ with $1 \mu \mathrm{m}$ in diameter heated by a $30 \mathrm{~mW} \mathrm{cw}$ laser spot with $15 \mu \mathrm{m}$ in diameter $(\mathrm{a}, \mathrm{b})$. Temperature of the layer stack (c). Adapted from [64]. 


\subsection{Experimental Results}

systems the power density dependent temperatures and temperature gradients as given in figure 2.11 can be assumed. For intensities higher than $40 \mathrm{~mW}$ both linear relations in figure 2.11 can be extrapolated.

As discussed above, the Seebeck coefficients are temperature dependent. Therefore, when calculating those from the measured voltages, not only the temperature gradient is of importance, but for comparison with the theoretical calculations also the higher temperature due to the heating needs to be taken into account.

\subsubsection{First Measured $U(H)$ Curve}

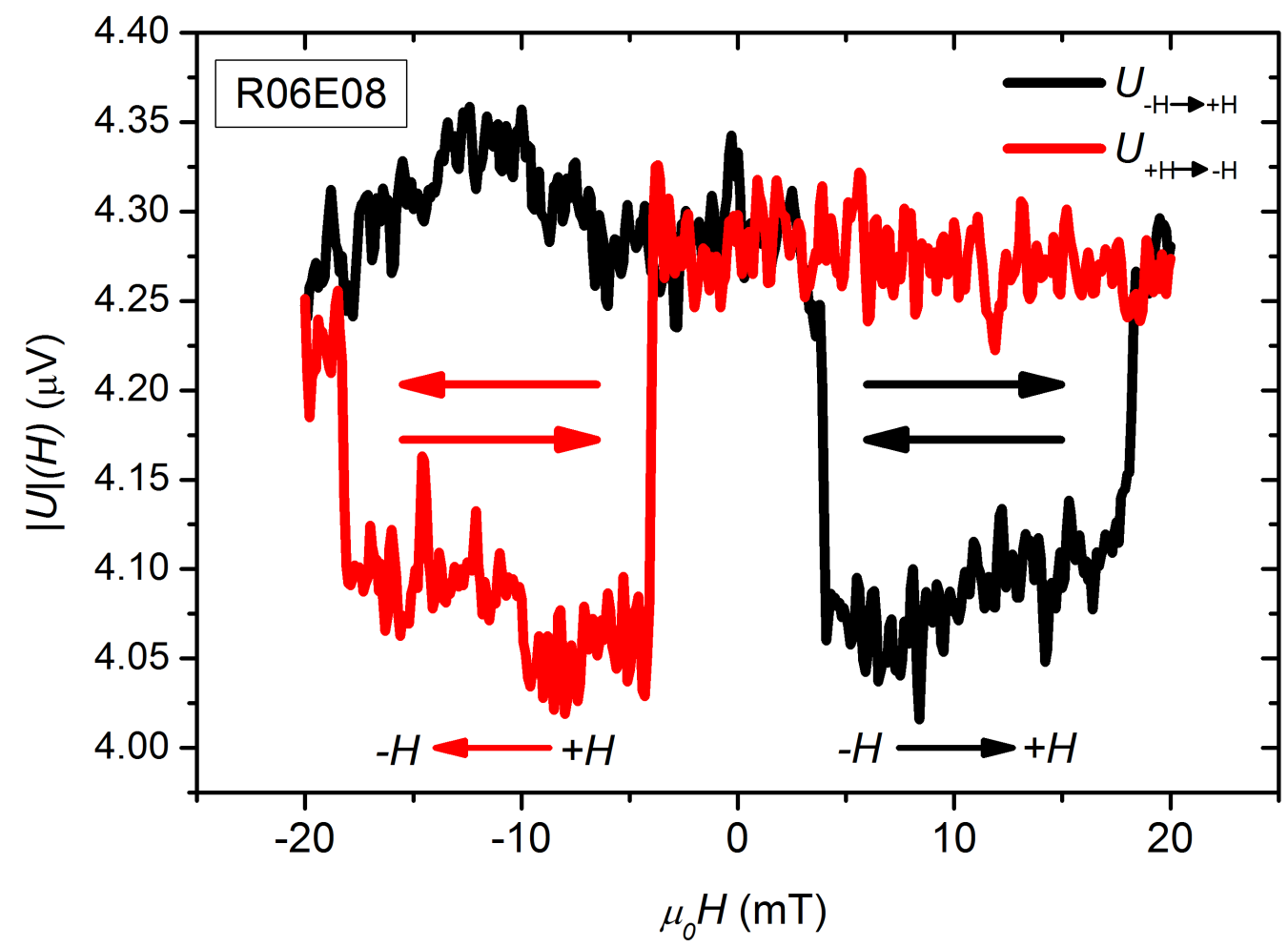

Figure 2.13: The first measured $|U|(H)$ curve showing a difference in voltage for parallel and antiparallel magnetization alignment and thus magneto-Seebeck effect. Measured in a junction with $2 \mu \mathrm{m}$ edge length (sample: mw100915b, R06E08).

Figure 2.13 shows the first measured curve in which the magneto-Seebeck effect can be distinguished in a $2 \times 2 \mu \mathrm{m}^{2}$ sized tunnel junction (sample mw101509b, element R06E08). The curve shows how with the reversing of the magnetization alignment from parallel to antiparallel the value of the voltage generated by the temperature gradient switches by $\Delta U \approx 0.25 \mu \mathrm{V}$. Starting with a negative applied field $\mu_{0} H=-20 \mathrm{mT}$ (black), both CoFeB layers' magnetization is aligned parallel 
$(|U|(H) \approx 4.3 \mu \mathrm{V})$. Decreasing the amplitude to $\mu_{0} H=0 \mathrm{mT}$, reversing its direction and increasing it again, slightly below $\mu_{0} H=5 \mathrm{mT}$, the top CoFeB layer's magnetization switches from pointing in the negative direction of $\mu_{0} H$ to the positive. At the same time, the voltage generated by $\nabla T$ jumps to $|U|(H) \approx 4.05 \mu \mathrm{V}$. The top CoFeB layer is thicker and has a lower coercive field than the bottom layer. Therefore, its magnetization follows the applied field for smaller values of $\mu_{0} H$. The magnetization alignment is antiparallel, until the bottom layer's magnetization also switches its direction at $\mu_{0} H \gtrsim 15 \mathrm{mT}$. The red part of the curve shows the same switching process, starting with a positive value of $\mu_{0} H$ and proceeding in the opposite direction.

The curve was recorded using the $\mathrm{cw}$ He-Ne laser at $6 \mathrm{~mW}$ as a heat source. Passing the beam splitter and the focusing optics, does not reduce the intensity of the laser beam significantly. Roughly one third of the light is absorbed by the $\mathrm{Au}$ capping layer. That means, the surface of the contact pad is heated by less than $1300 \mathrm{~W} / \mathrm{cm}^{2}$ for $0.625 \mathrm{~ms}$, given that the modulation frequency of the chopper is $800 \mathrm{~Hz}$. According to the simulations in [64 and figure 2.11, the temperature difference between the top and the bottom of the $\mathrm{MgO}$ layer is less than $10 \mathrm{mK}$ and the average temperature is less than $10 \mathrm{~K}$ above room temperature at this laser intensity. Together with the simulations for a $\mathrm{CoFeB}|\mathrm{MgO}| \mathrm{CoFeB}$ junction given in figure 2.20 this suggests a Seebeck voltage of at the most $(-0.35) \mu \mathrm{V}$ in the parallel state and remains at this order of magnitude independent of the CoFe structure at the $\mathrm{MgO}$ interface. Regardless of that, the measured data exhibits an absolute voltage that is an order of magnitude larger. These high background voltages may arise from additional temperature gradients at other interfaces, e.g. between the top layers. These additional temperature gradients can arise for two reasons. First, the junction is heated through the top layers, therefore temperature gradients can not be avoided there. However, the simulations of the temperature distribution [64, 69] do not point out temperature gradients, which might generate voltages that significantly contribute to the overall measured voltage. Second, if the impedance of the electric circuit is not high enough, electric currents will be induced by the generated Seebeck voltage and the Peltier effect can occur. According to [28], Peltier heating increases the temperature gradients at all interfaces of the layer stack at modulation frequencies around $1 \mathrm{kHz}$. All temperature gradients created at interfaces other than across the $\mathrm{MgO}$ tunnel barrier are not magnetization dependent and increase the overall measured voltage. This leads to a higher generated absolute voltage and the resulting magneto-Seebeck ratio appears smaller. To avoid these effects and the discrepancies to the simulations, later the impedance converter as explained in section 2.5.2 was installed. At this point, the sign of the voltage has not been determined, the procedure to determine the sign of the voltage is introduced in a section below. The magneto-Seebeck effect extracted from this data is around $6 \%$, although the simulation results shown in figure 2.20 suggest an effect of around $40 \%$ in this temperature region. Although the magneto-Seebeck effect appears small, the difference of the voltage between the parallel and antiparallel alignment $(\Delta U \approx 0.25 \mu \mathrm{V})$ is comparable to those measured in other junctions and mirrors the theoretically 


\subsection{Experimental Results}

predicted values for $\Delta U$ at $T \approx 310 \mathrm{~K}$ and $\nabla T \approx 10 \mathrm{mK}$. The theoretical model predicts $\Delta U=-0.2 \mu \mathrm{V}$ to $\Delta U=0.3 \mu \mathrm{V}$ for these temperatures and temperature gradients.

The noise in the measured data is ascribed to the instability of the He-Ne laser and the resulting irregularities in the heat distribution throughout the junction. To improve the signal to noise ratio in this experiment, for further measurements an intensity stabilized diode laser with adjustable output power has been installed.

\subsubsection{Temperature Dependent Seebeck Voltage in Large Sized Tunnel Junctions}

Installing the intensity stabilized diode laser and the impedance converter in the electric circuit enables fluence dependent Seebeck voltage measurements. The laser output power $P_{\text {Laser }}$ with intensities from $1 \mathrm{~mW}$ up to $30 \mathrm{~mW}$ used in the experiment, corresponds to optical densities from $100 \mathrm{~W} / \mathrm{cm}^{2}$ to $8000 \mathrm{~W} / \mathrm{cm}^{2}$, absorbed by the $\mathrm{Au}$ contact pad placed on top of the junction. A beam diameter of $15 \mu \mathrm{m}$ used in both, the simulations and the experimental data, makes the power densities directly comparable. The tunnel junction examined in this section, element R04E07 from the sample vz101007b, has the size $5 \times 5 \mu \mathrm{m}^{2}$. This junction was chosen, because despite its large area, it shows a high TMR effect around $140 \%$ and a clear switching between the parallel and antiparallel magnetization alignment, shown in figure 2.16. The resistance, of $12 \mathrm{k} \Omega$ in the parallel state is small compared to those of smaller sized junctions, in which the resistance is around $60 \mathrm{k} \Omega$.

The data are presented in figures 2.14 and 2.15(a). For these data the sign of the voltage was not determined, therefore only the absolute values will be discussed. Figure 2.14 shows that the voltage in the parallel magnetization alignment $U_{P}$ and in the antiparallel alignment $U_{A P}$ continuously increase with the laser power. Both voltages vanish when the laser power decreases towards zero. This shows that the voltage is solely generated by the temperature gradient $\nabla T$, which only occurs when laser heating is present. For low laser intensities $\left(P_{\text {Laser }} \leq 10 \mathrm{~mW}\right)$, the Seebeck voltage in the parallel alignment is low and the difference between the voltage in the parallel and antiparallel magnetization alignment is very small, therefore the magneto-Seebeck effect is not visible. In figure 2.15(a) left, the $|U|(H)$ curves are plotted in the range from $0-80 \mu \mathrm{V}$, the difference between the voltages in the parallel and antiparallel alignment is hardly visible in this depiction. Even the extraction of both voltages on the right does not show it clearer, until the difference $\left|U_{P}-U_{A P}\right|=\Delta U$ is plotted explicitly against $P_{\text {Laser }}$ (see inset in figure 2.15(a) right). The data shows that the difference between the voltage in the parallel and the antiparallel alignment increases steadily with the laser power, i.e. with increasing temperature and temperature gradient, but the order of magnitude is three times smaller than that of the voltages itself. The magneto-Seebeck ratio calculated from this data is shown in figure $2.15(\mathrm{~b})$, All $S_{M S}$ values are below $0.3 \%$. The simulations presented in [64] suggest a relative effect around 50\% at these temperatures and 


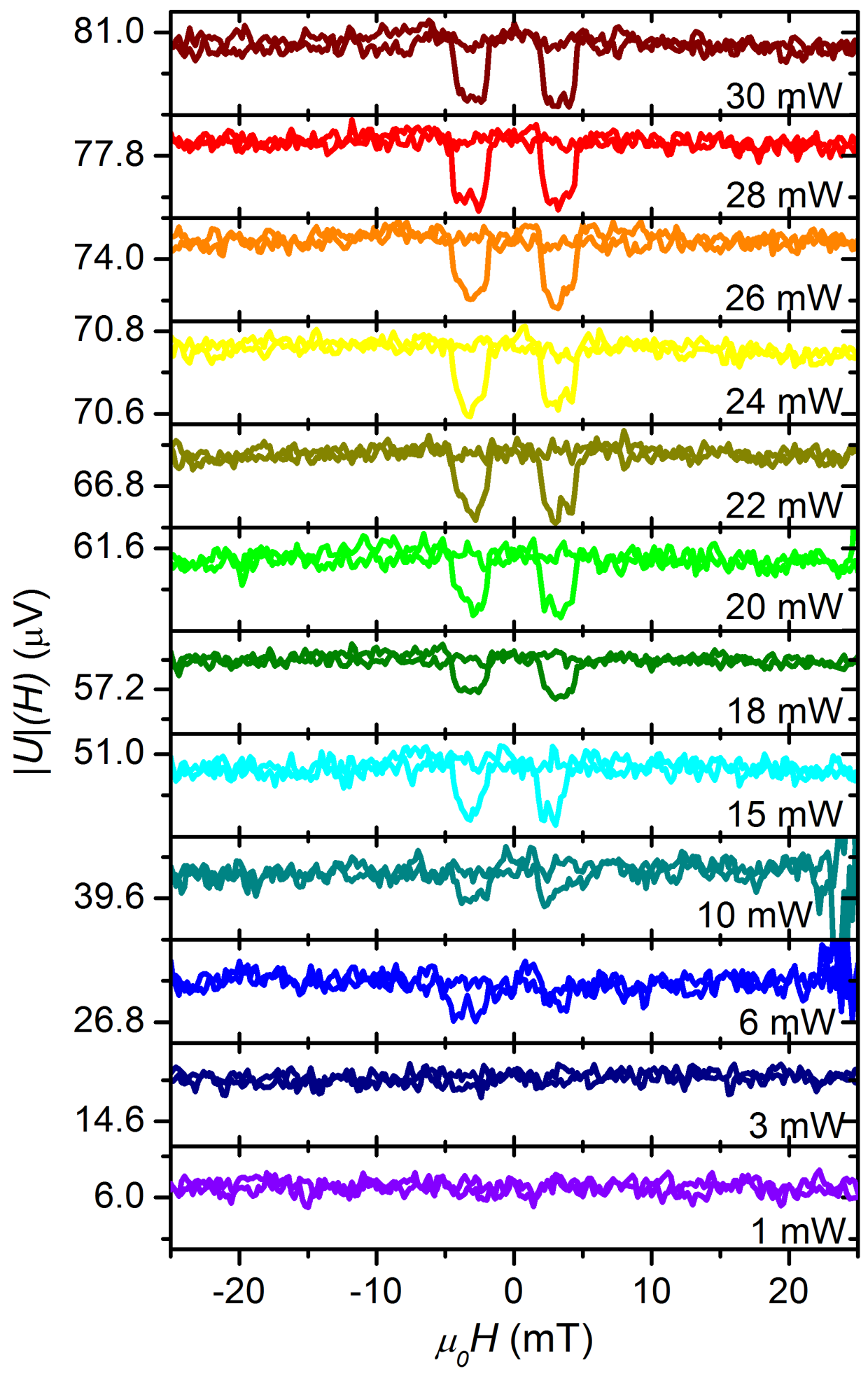

Figure 2.14: $|U|(H)$ curves measured using an intensity stabilized diode laser, increasing $P_{\text {Laser }}$ from $1 \mathrm{~mW}$ to $30 \mathrm{~mW}$. A voltage follower increased the impedance of the lock-in amplifier up to $100 \mathrm{G} \Omega$, assuring the vanishing of currents in the junction. 


\subsection{Experimental Results}

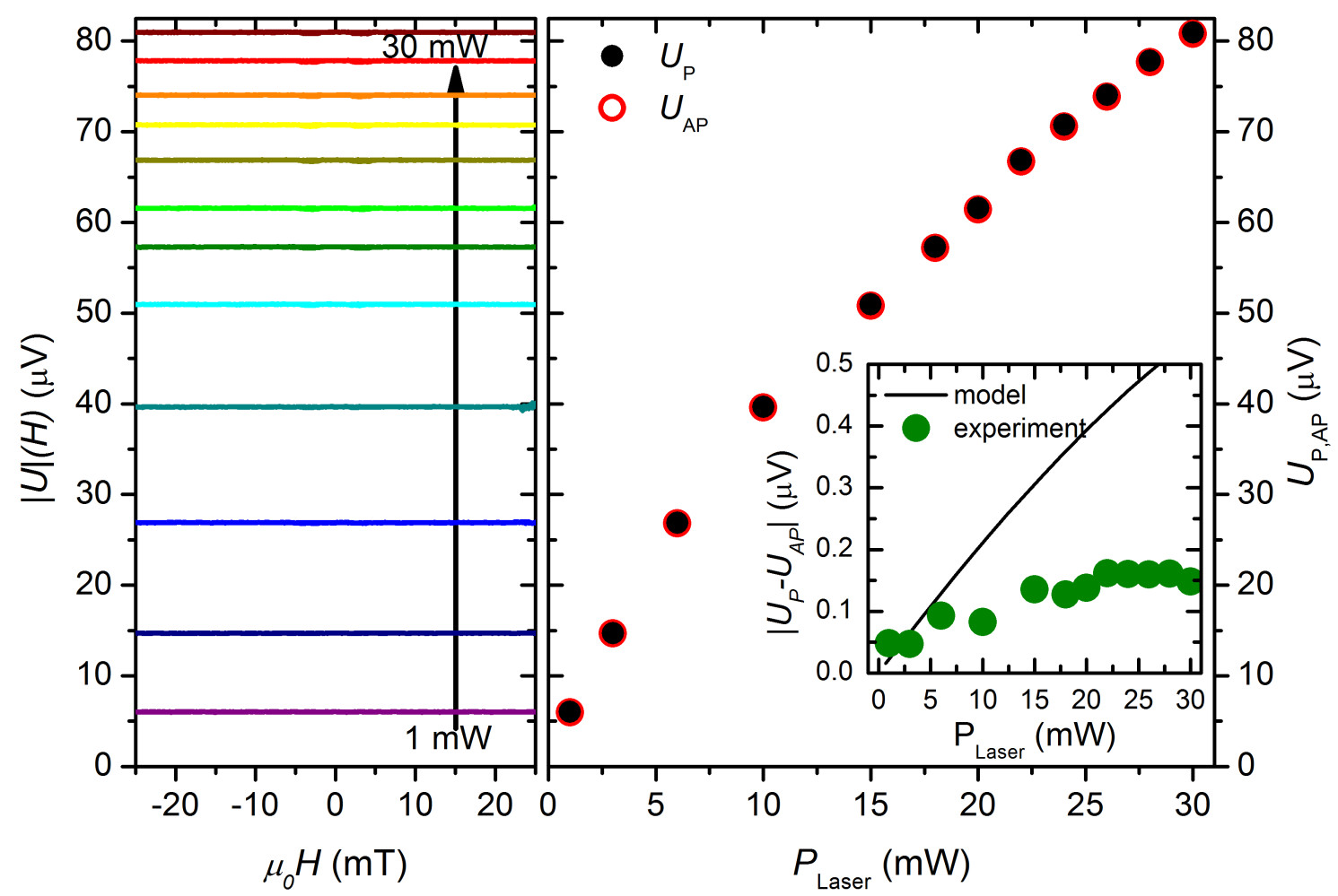

(a) Same $U(H)$ curves as in figure 2.14 (left). The junction shows a large voltage in the parallel $U_{P}$ (black dots) and antiparallel $U_{A P}$ (red circles) alignment compared to the difference $\left|U_{P}-U_{A P}\right|$ (green dots, inlet right). Both, $U_{P}$ and $U_{A P}$, increase nonlinearly with the laser fluence (right).

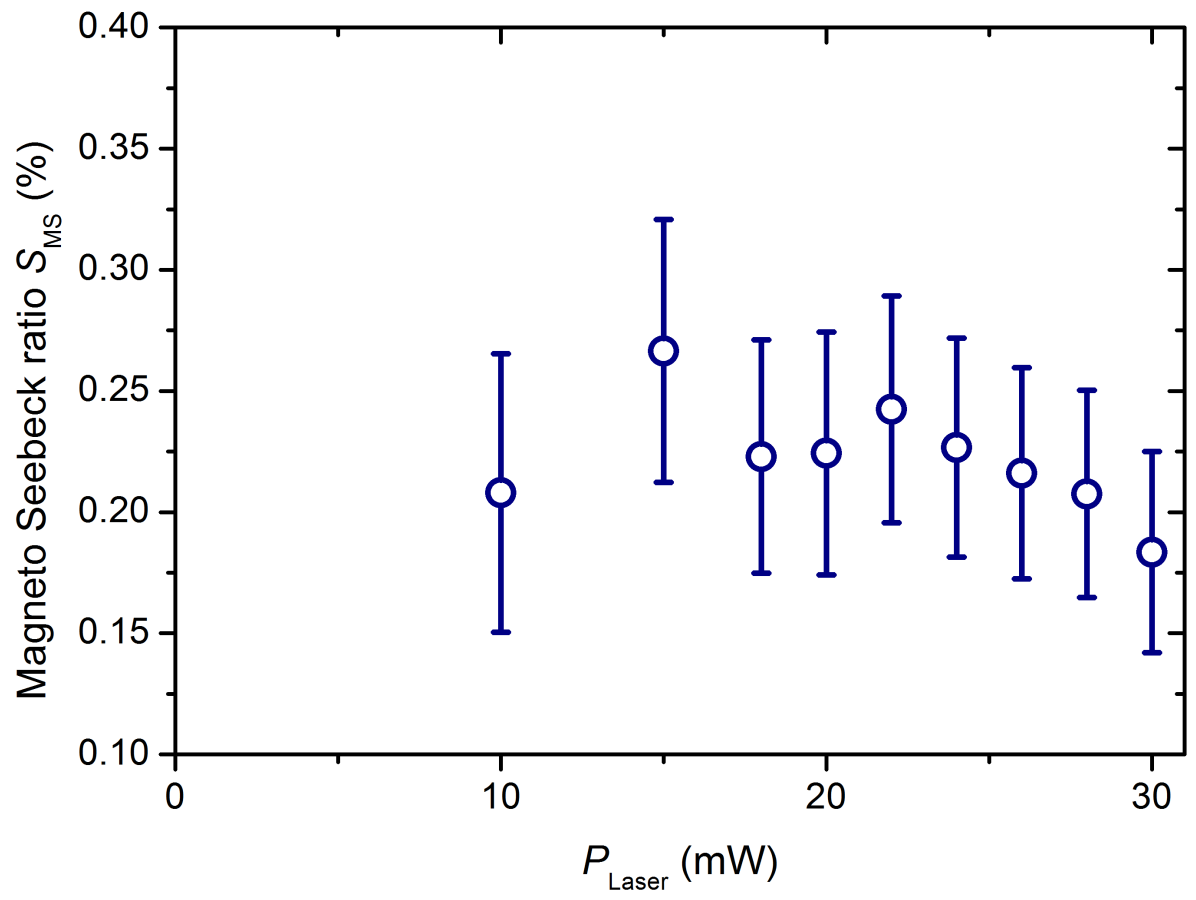

(b) Magneto-Seebeck ratio $S_{M S}$ calculated from data in figure 2.14

Figure 2.15: (a) $U(H)$ curves and the extracted voltages $U_{P}$ and $U_{A P}$. (b) The magnetoSeebeck ratio $S_{M S}$, plotted against the laser intensity. 


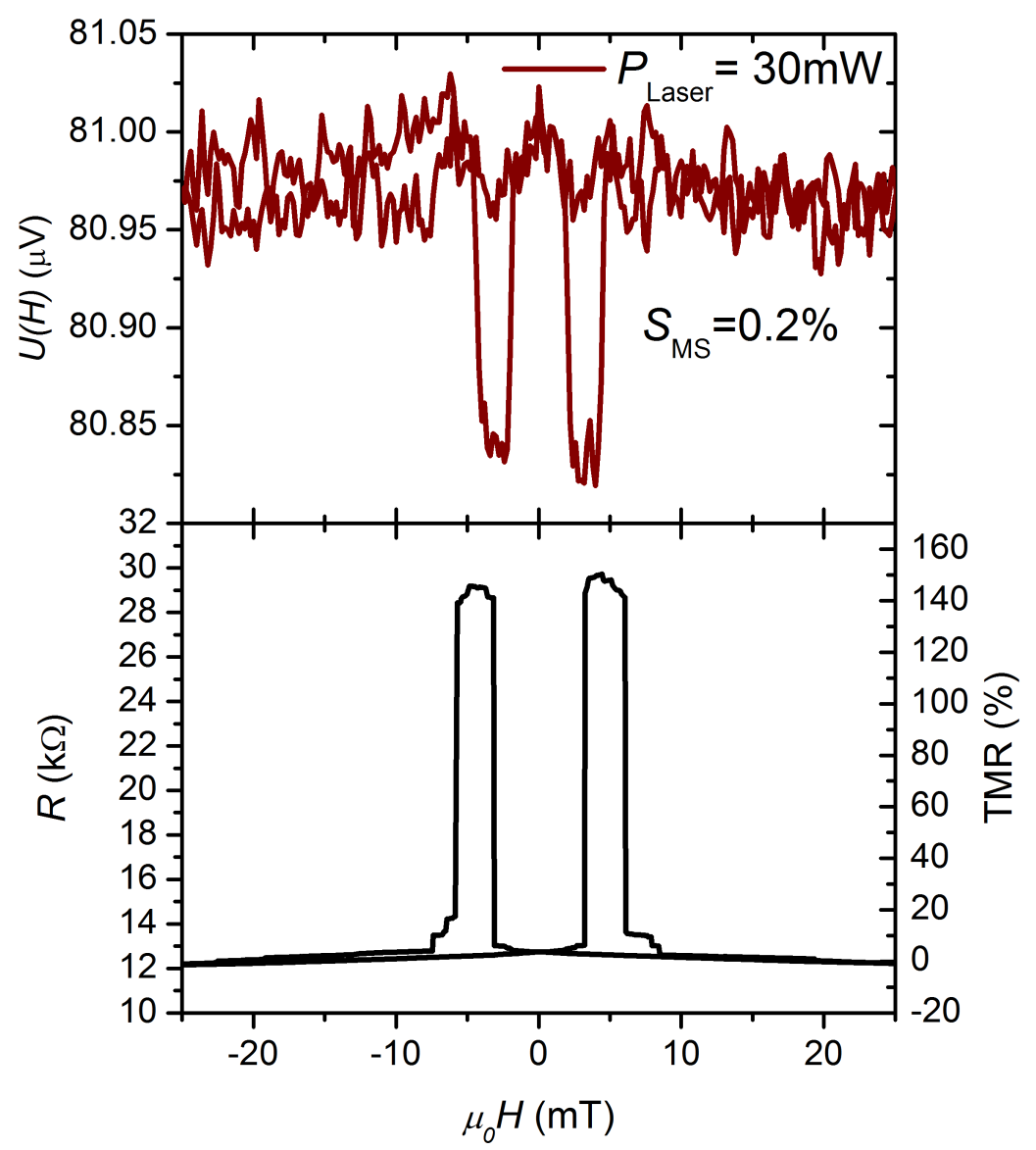

Figure 2.16: A very low relative magneto-Seebeck effect $0.2 \%$ (top) inspite of a high TMR effect of $140 \%$ and clean shifting between the parallel and antiparallel magnetization alignment (bottom) in the same tunnel junction.

much smaller voltages for these temperature gradients. However, the difference of the voltage $\Delta U$ is in the same order of magnitude as the theoretically predicted values, which are in the range from $\Delta U=0.05 \mu \mathrm{V}$ for the low laser powers and $\Delta U=0.5 \mu \mathrm{V}$ for $P_{\text {Laser }}=30 \mathrm{~mW}$, represented by the black line in the inset figure $2.15(\mathrm{a})$ right.

In spite of an intensity stabilized laser and the impedance converter in the electric circuit, still large background voltages are generated, which make the measured relative magneto-Seebeck ratio $S_{M S}$ appear small (see figure 2.15(b)). Because the absolute magneto-Seebeck effect, the difference between $U_{P}$ and $U_{A P}$, is of the same magnitude as the theoretically predicted values, the high background voltages are not a result of a malfunctioning MTJ, but must be generated elsewhere. Considering the rotational frequency of the chopper blade and the expansion of the laser beam, $33 \mu$ s are needed until the junction is illuminated homogeneously. In that time lateral temperature gradients can generate voltages at the intersections of the layers in the stack. These voltages are responsible for the high background voltage. Further 


\subsection{Experimental Results}

comparison to theoretical data shows that the magneto-Seebeck $S_{M S}$ ratio in figure 2.15(b) remains rather constant with increasing temperature. This behavior agrees with the theoretical model in figure 2.20. In that region, the magnetio-Seebeck ratio also shows a small slope.

In the following, smaller $1 \mu \mathrm{m} \times 1 \mu \mathrm{m}$ sized MTJ devices will be discussed and compared to the theoretically calculated data. There, the inhomogeneous heating and other effects discussed before, which may contribute to the background voltage, are further minimized. Before that, the modulation techniques and how the sign of the Seebeck voltage is determined will be discussed shortly.

\subsubsection{Modulation Techniques}

In general, increasing the modulation frequency reduces background noise, leading to better experimental results. Modulating the signal by a mechanical chopper limits the frequency to around $3 \mathrm{kHz}$, although theoretically $10 \mathrm{kHz}$ are possible. However, on the one hand, chopper blades with narrower slots require a very small laser beam waist for a clear distinction between the transmittance and non-transmittance periods. Otherwise high rise times for the laser intensity compared to the heating period emerge. On the other hand, wider slots require higher rotation frequencies, causing air turbulences and acoustic noise from vibrations, which couple into the experiment, amplifying the noise in the electric signal. High frequencies are required, because the resulting shorter heating periods prevent the sample from heating up at larger areas, reducing undefined voltages not generated across the tunnel junction. Figure 2.17 shows, how the increase of the modulation frequency improves the measured signal.

The gray and black curves show the signal arriving at the lock-in amplifier input for given modulation frequencies and were recorded from the monitor output. From these signals, the lock-in amplifier extracts the voltages at the junctions by Fourier transforming the signal and picking out the voltage at the given frequency and phase shift. The blue curves show the frequency of the laser light hitting the sample, centered around zero. At negative values, the laser is blocked by a slot of the chopper blade, whereas at positive values the sample is heated. This signal is recorded by a diode, which captures the light reflected from the sample. In the setup shown in figure 2.8, the diode is situated at the position of the CCD camera. The slow rise time is due to the expansion of the laser beam, when passing the chopper. A higher rotation frequency reduces this rise time, allowing a longer homogeneous heating periods and reducing side effects, like the expansion of the heat to larger areas.

There are mainly two effects, which significantly contribute to the improvement of the measured signal due to higher modulation frequencies.

The first effect is increasing the number of events locked by the lock-in amplifier per time unit, and thus reducing noise in the recorded signal. This results in a better distinction of the voltages in the parallel and antiparallel alignment of both magnetic layers in the junction.

The second effect is reducing the time span between blocking and unblocking 
the entire laser beam by the chopper, the opening period. During this period, the junction is heated homogeneously and especially for larger sized tunnel junctions it results in background voltages, as discussed in the previous section. In figure 2.17 it is indicated by the yellow lines and decreases from $100 \mu$ s for the modulation at $800 \mathrm{~Hz}$ to $50 \mu \mathrm{s}$ for the modulation at $3000 \mathrm{~Hz}$. The peaks in the measured curves, showing up immediately as heating starts, show a negative sign. When the heating stops, the voltage sign reverses. This happens, because the top layers of the sample have a higher heat conductance, and cool down faster, than the bottom layers, reversing the temperature gradient. At low modulation frequencies, when the slots shut the laser spot for long enough time periods, this sign reversed voltage relaxes to zero again. At high frequencies it does not.

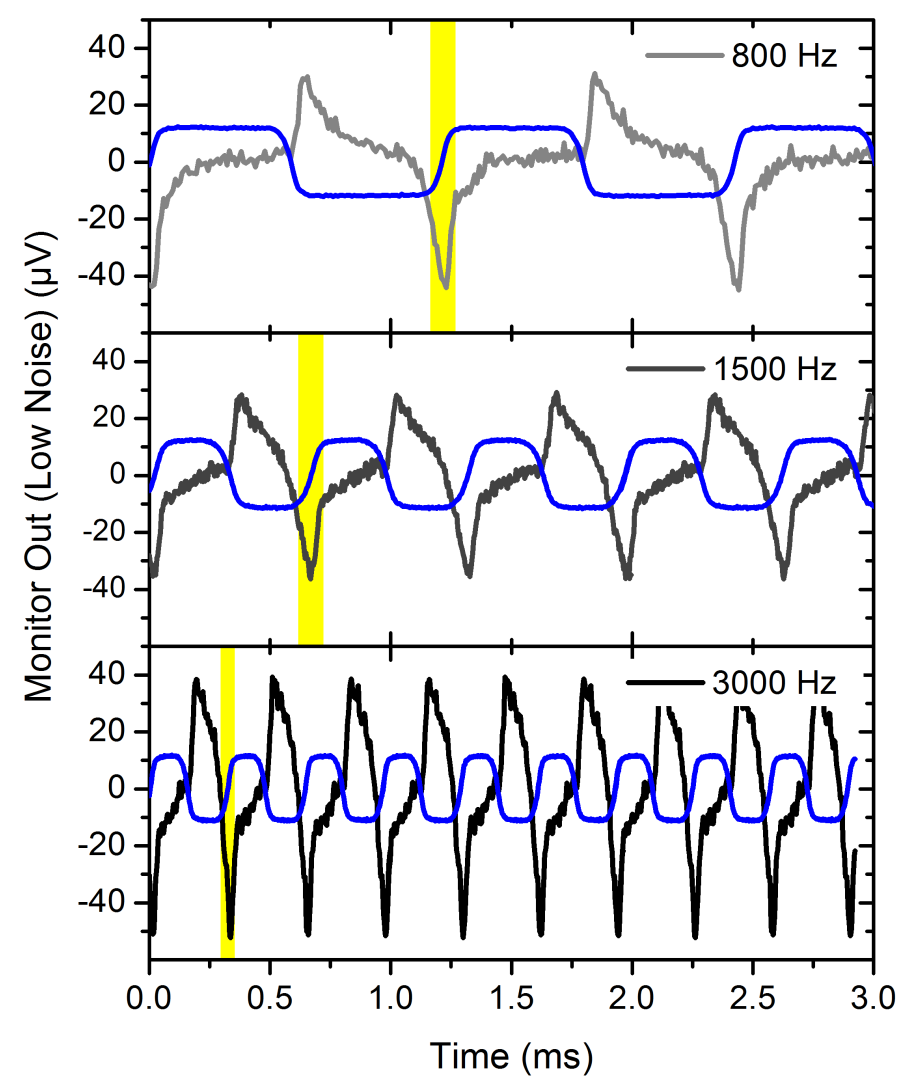

Figure 2.17: Increasing the modulation frequency, (shown from top to bottom) increases the events per unit time, over which the signal can be averaged. At higher frequencies the signal entering the lock in also increases.

For the final measurements, an asymmetric chopper blade, as shown in figure 2.9 , allowed short heating periods and enough time for relaxation. This blade, running at a frequency of $1.5 \mathrm{kHz}$, allows a heating time of $\sim 0.17 \mathrm{~ms}$ and a relaxation time of $\sim 0.5 \mathrm{~ms}$. This is the same time of heating, as when using a symmetric chopper blade, a chopper blade, where both slots have the same size, at a modulation frequency of $3 \mathrm{kHz}$, and a relaxation time at the frequency of $0.8 \mathrm{kHz}$. The slots are 


\subsection{Experimental Results}

wide enough to allow for lower rotation frequencies and as a consequence a reduced vibration noise coupled into the signal through acoustic waves. This alignment allows a measured signal with very low noise contribution and a clear distinction between the voltages in the parallel and antiparallel magnetization alignment of both magnetic layers.

\subsubsection{Determining the Sign of the Seebeck Voltage}

The lock-in amplifier records the amplitude and the phase of the voltage generated at the tunnel junction. However, the Seebeck coefficients of tunnel junctions can also have negative values resulting in negative voltages. Therefore a thorough data analysis for the determination of the voltage sign is essential.

The sign of the voltage can be determined from the temporal traces of the lock-in amplifier signal input. As an example, figure 2.18 shows the temporal traces of the voltage across a junction recorded at a modulation frequency of $1.5 \mathrm{kHz}$ using the asymmetric chopper blade. The voltages measured at the junction are shown by black lines for parallel magnetization alignment and by red lines for the antiparallel magnetization alignment. The temporal traces are shown for six different laser intensities, increasing from $10 \mathrm{~mW}$ to $150 \mathrm{~mW}$. These traces were recorded using a pulsed femtosecond laser, delivering enough energy to heat the junction to temperatures, at which the magneto-Seebeck ratio changes sign, which is further discussed below in section 2.6.6. The blue line shows the signal recorded by the control diode. The hatched area in the background represents the time period, when the chopper blade is blocking the laser beam, whereas the white background shows the time, when the chopper is open for the laser beam. The change in background also matches the rectangle function generated by the chopper electronics, by which the lock-in amplifier is modulated.

The diode signal shows that the spacial extension of the beam softens the sharp modulation signal, starting to heat the sample, shortly before the chopper blade allows the entire laser beam to pass through, the heating profile follows an error function $(\operatorname{erf}(x))$. The voltage generated by the heat distribution caused by the light deposited at the sample surface is included in the negative peak showing first at around $0.6 \mathrm{~ms}$ and then every $0 . \overline{6} \mathrm{~ms}$. From this peak it is concluded that the voltage generated across the tunnel junction is negative. This peak shifts to smaller times, as the laser intensity increases. This is clear, since for larger intensities, more power is included into the peripheries of the beam waist, which heats up the sample earlier also indicated by the diode signal, which does not mirror an exact rectangular function. At low intensities, both curves, in the parallel and antiparallel alignment, do not show significant changes, because for the temperatures reached across the tunnel junction by these intensities, only a small magneto-Seebeck ratio is attained. However, with increasing laser intensity, a dip in the temporal trace for for both magnetization alignments shows up, growing towards positive voltages. The larger amplitude of the dip in the antiparallel magnetization alignment is an indication for 


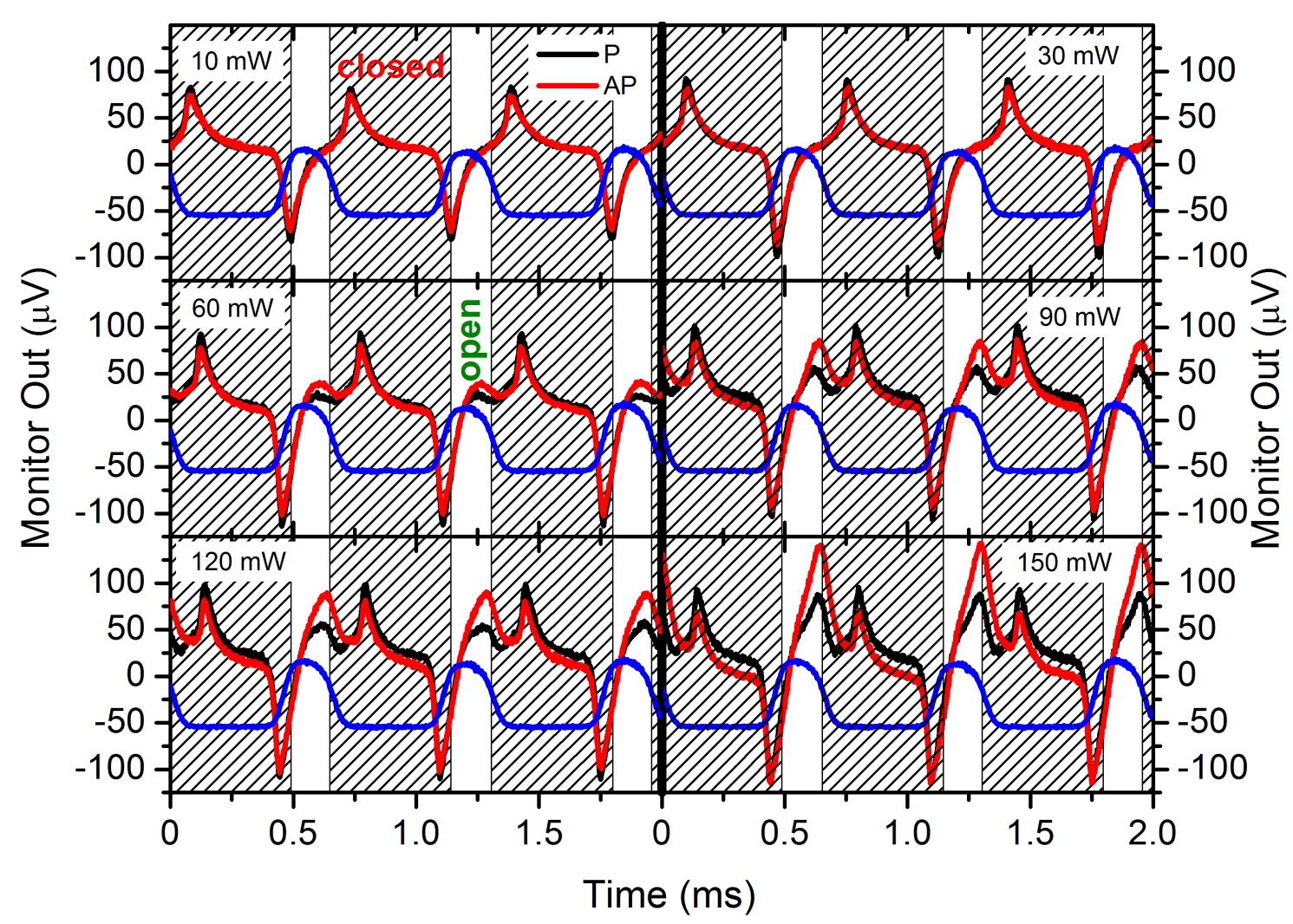

Figure 2.18: Temporal traces recorded from the lock-in amplifier monitor output. The black lines show the signal obtained by the lock-in amplifier, when both magnetic layers magnetizations are aligned parallel $(\mathrm{P})$, and the red lines show the signal in antiparallel (AP) alignment. The blue lines show the control diode signal and indicate, when the sample is heated, and when the laser beam is blocked by the chopper blade. The white (open) and hatched (closed) background shows the chopper reference signal. With an increasing pump fluence, a change of sign in the magneto-Seebeck effect becomes visible.

reversal of the Seebeck coefficients' values for each magnetization alignment.

The lock-in amplifier picks the first harmonic frequency $\omega$ and its phase from the modulated signal that is recorded and can be observed in the Monitor-Out output, referred to as temporal traces. An FFT analysis of these temporal trace signals, disentangles the amplitudes and frequencies included in the recorded signal in the same way as the lock in amplifier. Plotting the amplitude with the corresponding phase shift information extracted by the FFT analysis at the modulation frequency as a simple harmonic function, e.g. the sine function (see figure 2.19), reveals that at the time around $0.6 \mathrm{~ms}$, after the chopper has opened and the junction has been heated up, the voltage amplitudes are negative. There is, however, a difference between the values in the parallel and antiparallel alignment. This difference corresponds to the values shown in the applied field dependent measurements, in section 2.6.6. 


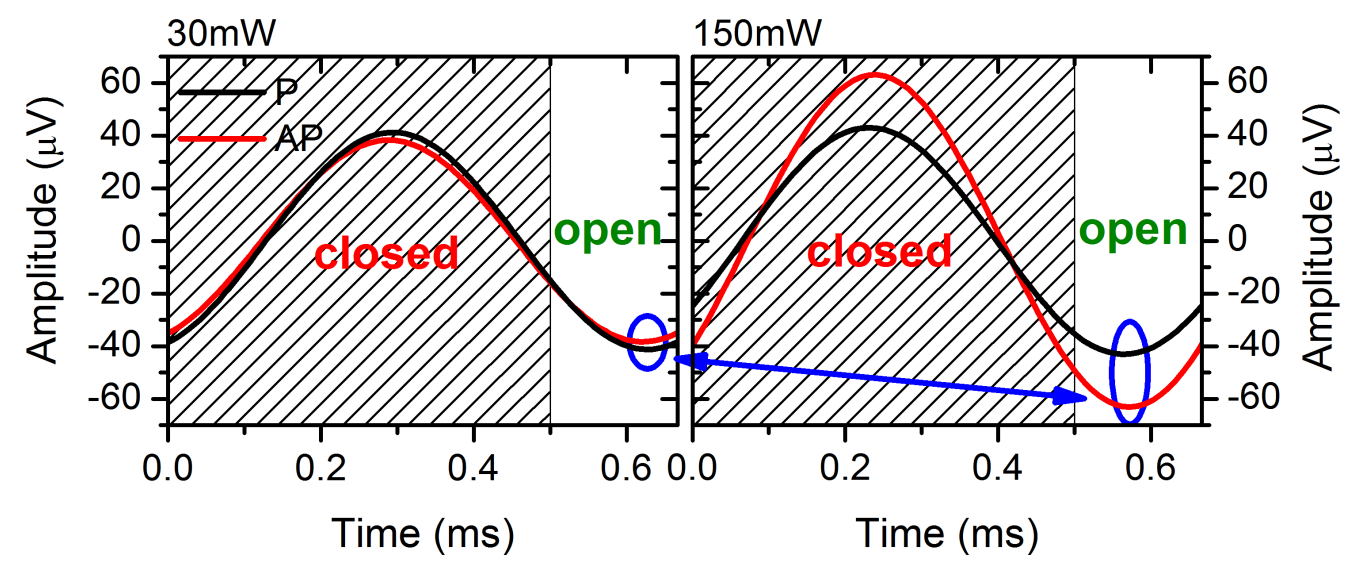

Figure 2.19: Sine waves with amplitudes and phase shifts extracted from temporal traces in figure 2.18 using FFT. At $0.5 \mathrm{~ms}$ the voltage is negative, and there is an inversion of the amplitude ratio, between low and high laser intensity, when the magnetization switches from from parallel to antiparallel alignment. This leads to a sign change in the magneto-Seebeck ratio.

As indicated by the blue circle, the amplitudes of the voltages in the parallel and antiparallel alignment change their ratio at low intensity and high intensity respectively. This is in agreement with the simulations given in [64], discussed in section 2.6 .6 ,

\subsubsection{Final Experimental Results and Simulations}

To compare the experimental results measured on the smallest element sized $1 \times$ $1 \mu \mathrm{m}^{2}$ with theoretical calculations by Christian Heiliger's group published in [64, 17], first, their results will be presented. They perform ab initio calculations using the energy dependent transmission function $T_{t}(E)$ for the transmission probability of electrons through a tunnel barrier based on the Green's function formalism engaged into the Korringa-Kohn-Rostoker [30] method described in section 2.4.3. Figure 2.20 shows the calculated Seebeck coefficients in the parallel $S_{P}$ and the antiparallel $S_{A P}$ magnetization alignment and the resulting magneto-Seebeck ratio $S_{M S}$ of a MgO-based tunnel junction in the temperature range from $100 \mathrm{~K}-800 \mathrm{~K}$. These coefficients are calculated for 10 monolayer $\mathrm{MgO}$ tunnel barriers between 20 monolayer thick bcc structured $\mathrm{Co}_{50} \mathrm{Fe}_{50 \%}$ magnetic layers, and semi-infinite bcc structured $\mathrm{Cu}$ leads [64]. There are several possibilities, how the same number of Co and Fe atoms can be distributed in the bcc structure. The results shown in figure 2.20 are simulated for an even distribution at the $\mathrm{MgO}$ interface, as depicted in figure 2.21, which is referred to as the alternating-termination structure. In the following that means that in each CoFe layer, every Co atom has Fe atoms as next neighbors and vice versa. For this composition, the calculated charge-Seebeck coefficients are negative for both magnetization alignments in the temperature range from $100 \mathrm{~K}$ to 


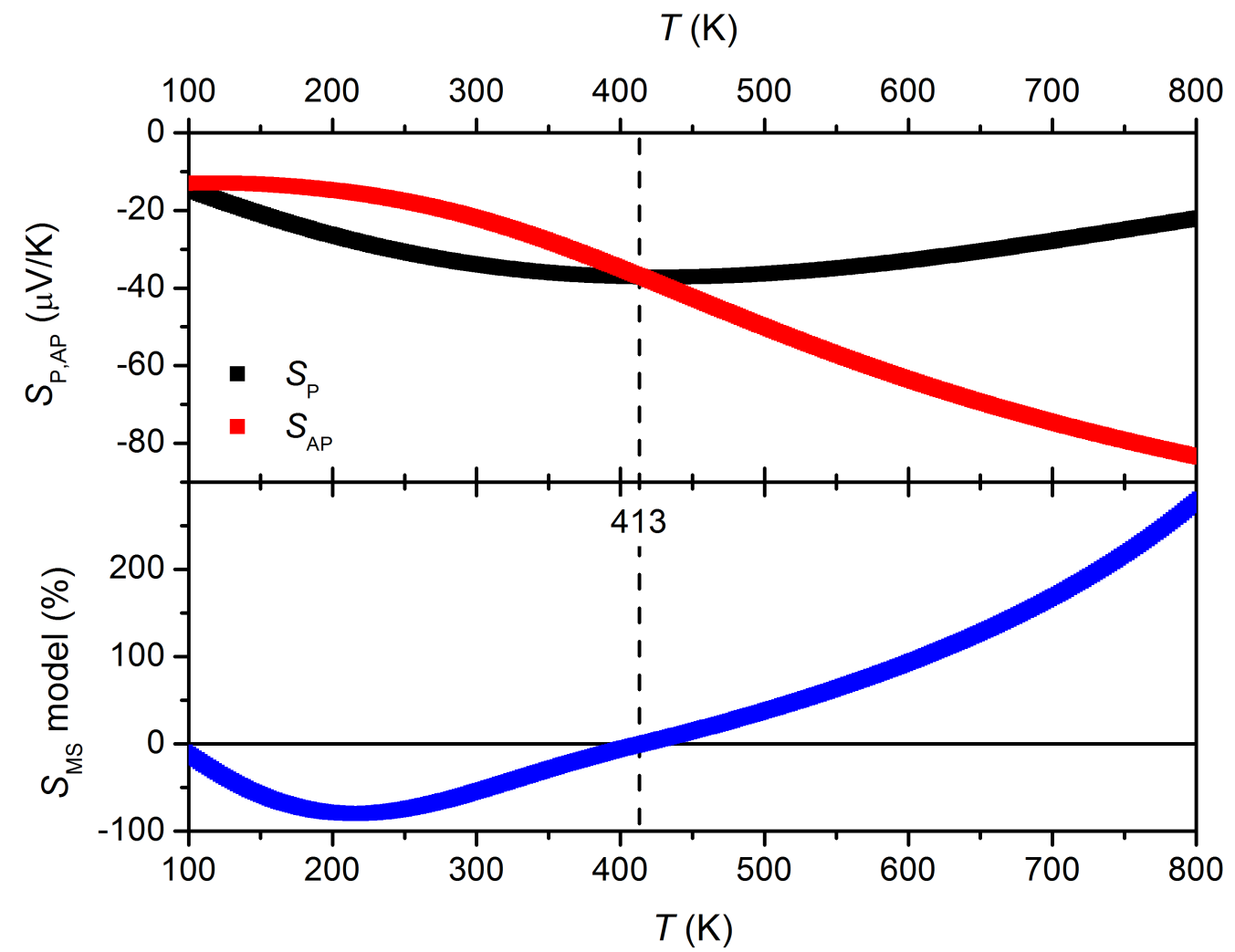

Figure 2.20: Magneto-Seebeck coefficients for the parallel $S_{P}$ and the antiparallel $S_{A P}$ magnetization alignment of both magnetic layers obtained from ab-initio calculations using the transmission function $T_{t}(E)$ (upper graph), and the magneto-Seebeck ratio calculated from these coefficients as function of temperature $T$ (lower graph). Taken from [64].

$800 \mathrm{~K}$, but their absolute values change. For temperatures below $413 \mathrm{~K}$, the Seebeck coefficient in the antiparallel magnetization alignment $S_{A P}$ has a smaller absolute value than in the parallel alignment $S_{P}$. This circumstance reverses for temperatures above $413 \mathrm{~K}$ and leads to a sign change in the magneto-Seebeck ratio $S_{M S}$ at $413 \mathrm{~K}$.

Because of coherent tunneling, the values of the Seebeck coefficients strongly depend on the CoFe composition and the atomic structure at the $\mathrm{MgO}$ interface. To reveal the differences, Seebeck coefficients for the parallel and antiparallel magnetization alignment for various $\mathrm{Co} F$ distributions at the $\mathrm{MgO}$ interface at $300 \mathrm{~K}$ have been calculated for a $\mathrm{CO}_{50 \%} \mathrm{Fe}_{50 \%}$ composition in [64], the results are summarized in table 2.1. For the alternating termination structure depicted in figure 2.21, the coefficients are $S_{P}=-34.0 \mu \mathrm{V} / \mathrm{K}, S_{A P}=-21.9 \mu \mathrm{V} / \mathrm{K}$, and the magneto-Seebeck ratio is $S_{M S}=-55 \%$. Turning both CoFe layer stacks in figure 2.21 by $90^{\circ}$ results in a distribution of alternating Co and Fe layers. There can be either a Co layer at the 


\subsection{Experimental Results}

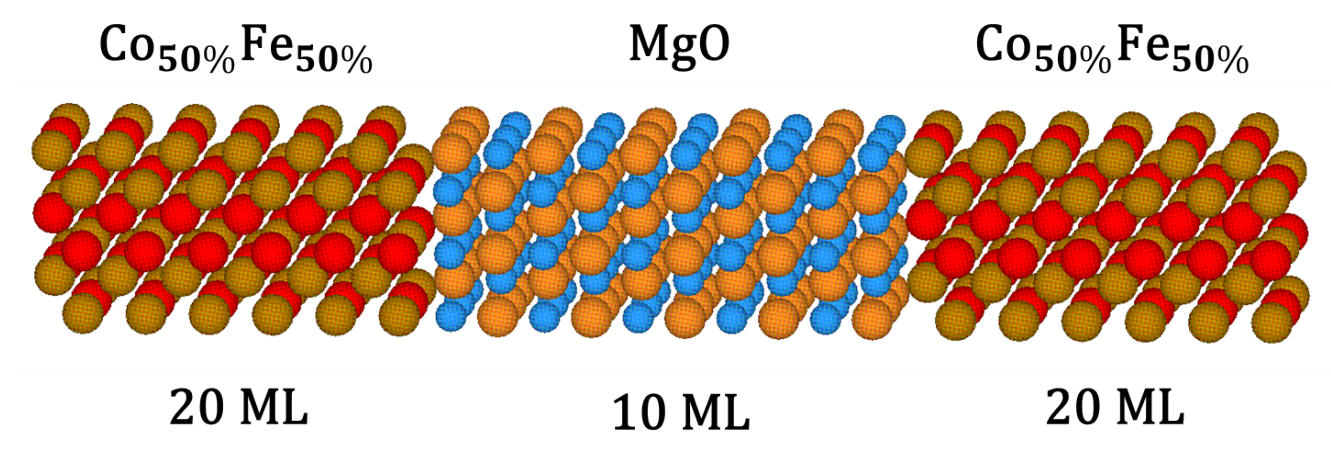

Figure 2.21: Co and Fe distribution in bcc structure at a 10 monolayer (ML) MgO barrier interface (alternating-termination structure). This structure is chosen for simulations resulting in the data shown in figure 2.20 64.

$\mathrm{MgO}$ interface, referred to as the Co-termination structure, or a Fe layer, referred to as the Fe-termination structure. For a Co-termination structure, the calculated coefficients are $S_{P}=-19.7 \mu \mathrm{V} / \mathrm{K}, S_{A P}=-32.4 \mu \mathrm{V} / \mathrm{K}$, and the magneto-Seebeck ratio is $S_{M S}=64.1 \%$. In that case, only the absolute values of the coefficients change at magnetization reversal, but the signs remain the same. The magneto-Seebeck ratio has a positive value for this structure, because the ratio between $S_{P}$ and $S_{A P}$ is inverted to that in the alternating-termination structure. For the Fe-termination structure, the calculated coefficients are $S_{P}=45.9 \mu \mathrm{V} / \mathrm{K}, S_{A P}=-50.0 \mu \mathrm{V} / \mathrm{K}$, and the magneto-Seebeck ratio is $S_{M S}=209.0 \%$. In that case only the Seebeck coefficient in the parallel magnetization alignment is positive, resulting in a positive magneto-Seebeck ratio.

\begin{tabular}{|l||r|r|r|}
\hline magnetic layer structure & $S_{P}\left(\mu \mathrm{VK}^{-1}\right)$ & $S_{A P}\left(\mu \mathrm{VK}^{-1}\right)$ & $S_{M S}(\%)$ \\
\hline \hline alternating-termination & -34.0 & -21.9 & -55.2 \\
\hline Co-termination & -19.7 & -32.4 & 64.1 \\
\hline Fe-termination & 45.9 & -50.0 & 209.0 \\
\hline pure Fe-lead & $\approx-14$ & $\approx 6$ & $\approx 330$ \\
\hline
\end{tabular}

Table 2.1: Seebeck coefficients in the parallel an antiparallel magnetization alignment at $300 \mathrm{~K}$ for different bcc-CoFe structures at the $\mathrm{MgO}$ interface, taken from 64. and for a pure bcc-Fe lead at the $\mathrm{MgO}$ interface extracted from graphs in [17].

The characteristics in the Seebeck coefficients are ascribed to the electronic band structures resulting from the different combinations of Co and Fe atoms at the $\mathrm{MgO}$ interfaces. Pure bcc-Fe has a lower Fermi energy with respect to the bottom edge of the majority-spin $\Delta_{1}$ band than bcc-Co. Therefore, Fe has a larger number of electron bands at the Fermi level, which are able to contribute electrons into the conduction band, when their energy is slightly elevated, e.g. due to a temperature rise. Therefore the calculated charge-Seebeck coefficient for pure Fe is larger than for Co for temperatures below $550 \mathrm{~K}$. Both coefficients are positive in this temperature 
range, see figure 2.6 (left). That means, electrons from the warmer region move to the colder region resulting in a positive voltage. However, it is not trivial to clarify, how the charge-Seebeck coefficients and their signs for tunnel junctions in the parallel and antiparallel magnetization alignment occur. Considering different Co and $\mathrm{Fe}$ compositions at the $\mathrm{MgO}$ interface and with it slightly shifted $E_{F}$ and band structure cannot explain the differences that occur in the Seebeck coefficients.

Bringing up the pure band structure to estimate the Seebeck coefficients for MTJs with pure bcc-Fe magnetic layers, one would expect positive $S_{P}$ values, because there should be enough electrons in the conduction band to tunnel through the $\mathrm{MgO}$ layer from the warmer magnetic layer to the colder layer. At the same time, if the tunnel barrier is a perfect spin filter, a negative $S_{A P}$ should be possible, because the excited electrons from the warmer layer are not able to tunnel through the $\mathrm{MgO}$ layer to the colder region, resulting in a negative voltage. However, at $300 \mathrm{~K}$, both Seebeck coefficients obtained using the model introduced in section 2.4.3 have the opposite signs [17]. In general, the calculated $S_{P}$ for MTJs with pure bcc-Fe magnetic layers has a negative sign in the temperature range from $0 \mathrm{~K}$ to $1000 \mathrm{~K}$, while $S_{A P}$ changes sign two times (see figure 2.6 right). Apart from that for Fe-termination structured magnetic layers, one would expect a similar band structure to that of pure bcc-Fe magnetic layers, with a slightly elevated Fermi energy level $E_{F}$, due to the Co atoms underneath the Fe layer, and therefore positive $S_{P}$ values and possibly negative $S_{A P}$ values. In this case, at $300 \mathrm{~K}$ the calculated Seebeck coefficients agree with this simple consideration. However, the opposite signs of $S_{P}$ and $S_{A P}$ for MTJs with pure bcc-Fe magnetic layers and the Fe-termination structure magnetic layers are not explainable from this simple picture.

With increasing relevance of the Co atoms at the interface to the $\mathrm{MgO}$ tunnel barrier, one would expect the Seebeck coefficients to become smaller than for Fetermination or pure bcc-Fe magnetic layers, as with higher Co content, the number of conduction electrons decreases. Further, a sign change would be expected around $550 \mathrm{~K}$, since there the Seebeck coefficient of pure bcc-Co also changes sign. In this case, the calculated $S_{P}$ and $S_{A P}$ for the alternating termination structure are negative in the whole temperature range from $0 \mathrm{~K}$ to $1000 \mathrm{~K}$ (see figure 2.20). Additionally, both Seebeck coefficients for the Co-termination structure are negative at $300 \mathrm{~K}$. This indicates, that not only the resulting electronic band structure at the $\mathrm{MgO}$ interface is relevant for the Seebeck coefficients, but also the electronic band structure in the remaining parts of the magnetic layers and the properties of the tunnel barrier.

This variety of Seebeck coefficients opens possibilities to tailor materials with desired Seebeck coefficients. The control over these parameters will enable the production of $\mathrm{MgO}$-based storage devices, requiring a minimum of energy to determine the magnetization alignment, and thus to read out the recorded information. The control over $S_{P}$ and $S_{A P}$ will be gained solely by providing the right band structure through the control over the ratio and distribution of $\mathrm{Co}$ and $\mathrm{Fe}$ atoms at the $\mathrm{MgO}$ interface.

The experimental methods to determine the Co and Fe distribution of the lay- 


\subsection{Experimental Results}

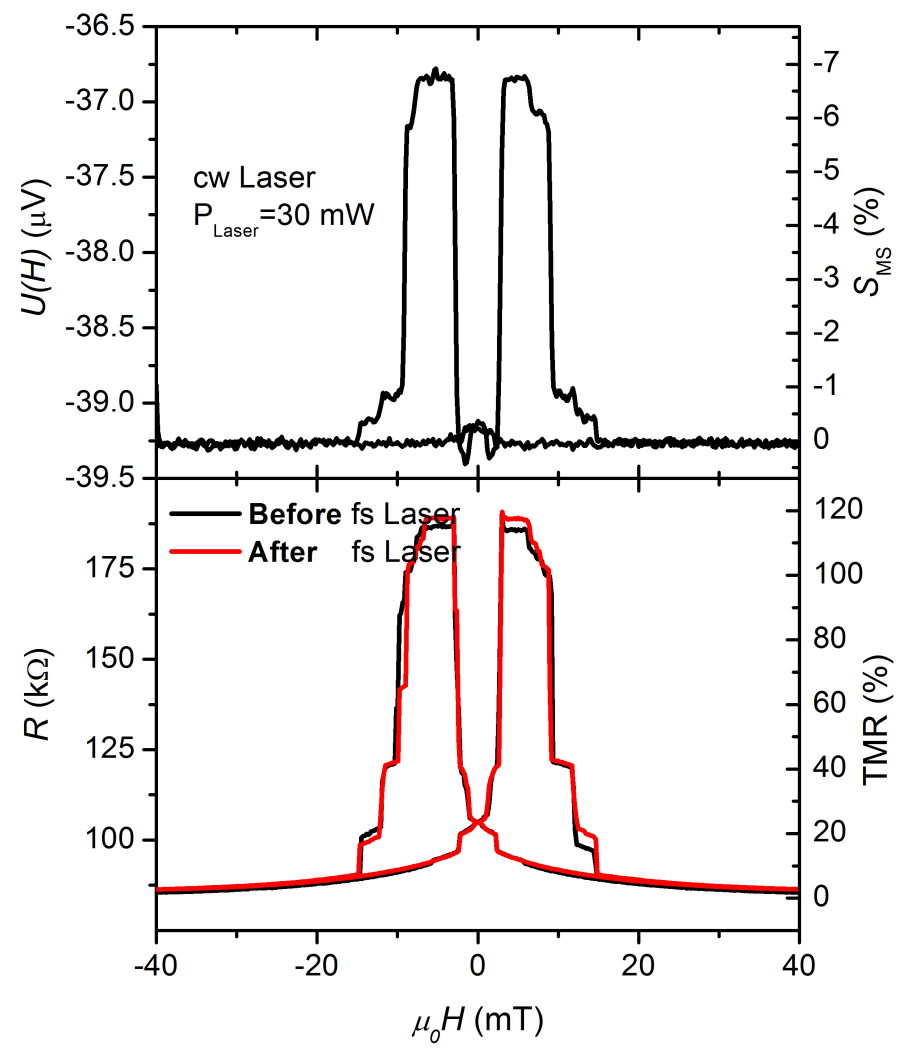

Figure 2.22: Magneto-Seebeck curve measured using a cw diode laser, shows an effect of $S_{M S}=-6.8 \%$. The TMR curves were measured before and after heating the junction with high intensities of the pulsed fs laser. The TMR remains solid at around $120 \%$. This demonstrates that the junctions has not been damaged by these high fluences.

ers at the $\mathrm{MgO}$ interface are rather limited. High-resolution transmission electron microscopy is capable of determining the crystal structure, but can not distinguish between $\mathrm{Co}$ and $\mathrm{Fe}$ due to the similar masses. In the future, samples for a field ion microscope FIM can be prepared and evaporated in the tomographic atom probe TAP. This technique can distinguish atoms even if their masses have the difference of $1 \mathrm{u}$ [51]. This method would deliver the ultimate experimental proof for the theoretical predictions. Until these investigations have been done, and the junction production processes are mature enough to deliver the desired distributions, the analysis of the voltage sign, or the sign of the Seebeck coefficient respectively, will be helpful to estimate the atom distributions based on the data from the ab-initio simulations.

The knowledge gained from previously discussed experiments and an optimized setup lead to the choice of a tunnel junction of the smallest possible size that shows a high TMR ratio and a clean switching behavior from the parallel to the antiparallel magnetization alignment. A small size usually means high resistance in the 
parallel magnetization alignment and enables homogeneous heating, which eliminates background voltages. A clean switching means, the high resistance in the antiparallel state is reached within a small range of the external field $\Delta H<0.2 \mathrm{mT}$ and can be maintained, while the external field is shifted by about $5 \mathrm{mT}$. Element R07E02 $\left(1 \times 1 \mu \mathrm{m}^{2}\right)$ from sample vz101007b shows a high resistivity in the parallel alignment, around $90 \mathrm{k} \Omega$, and a high TMR ratio around $120 \%$. The TMR ratio and high resistivity kept their values, even after heating the junction with the high intensities of the pulsed fs laser. This assures that the junction has not been damaged during these experiments (see figure 2.22). The data was recorded using the asymmetric chopper blade set to a modulation frequency $f_{\bmod }=1.5 \mathrm{kHz}$. First, $U(H)$ curves were recorded using the $\mathrm{cw}$ diode laser, increasing the laser power from $5 \mathrm{~mW}$ to $40 \mathrm{~mW}$. This corresponds to optical densities absorbed by the sample from $\sim 1000 \mathrm{~W} / \mathrm{cm}^{2}$ to $\sim 8000 \mathrm{~W} / \mathrm{cm}^{2}$, reaching temperature gradients from $\Delta T=8.76 \mathrm{mK}$ up to $\Delta T=70.51 \mathrm{mK}$, at temperatures between $T=299 \mathrm{~K}$ and $T=340 \mathrm{~K}$ for the lowest and highest intensities respectively. The simulated data in figure 2.11 show a linear dependence on the laser intensities in this range. According to the data simulated in [64] for the alternating-termination structure at the $\mathrm{MgO}$ interface, there is no sign change expected between $S_{P}=-33.9 \mu \mathrm{V} / \mathrm{K}$ up to $S_{P}=-35.6 \mu \mathrm{V} / \mathrm{K}$ and $S_{A P}=-21.9 \mu \mathrm{V} / \mathrm{K}$ up to $S_{A P}=-26.6, \mu \mathrm{V} / \mathrm{K}$ in this temperature range. The difference is only $\sim 1.7 \mu \mathrm{V} / \mathrm{K}$ and $\sim 4.7 \mu \mathrm{V} / \mathrm{K}$, respectively. The magneto-Seebeck ratio is predicted around $S_{M S}=-55 \%$, decreasing down to $S_{M S}=-34 \%$ with increasing temperature.

\section{Temperature Dependent Experiments using the cw Laser}

Figures 2.23 and 2.24 show the measured $U(H)$ curves. At first view, the data contains less noise than the data presented previously in figures 2.14 and 2.15(a). Further, this data shows lower absolute voltages in the parallel alignment $U_{P}$ and obviously a higher magneto-Seebeck ratio. The right graph in figure 2.23 shows voltages in the parallel $U_{P}$ (filled circles and triangles) and the antiparallel $U_{A P}$ (open circles and triangles) magnetization alignment of the junction. The circles show the values determined from experimental data plotted against laser intensity, extracted from the $U(H)$ curves shown on the left. The triangles show voltages calculated based on the theoretical Seebeck coefficients $S_{P}$ and $S_{A P}$ (see figure 2.20), and the temperature gradients $\Delta T$ determined in simulations (see figure 2.11). The temperature on the top $\mathrm{x}$-axis is calculated using the data in figure 2.11 .

The theoretical calculations suggest a nearly linear dependence of both voltages in this temperature range, given that the temperature gradient also increases linearly with the temperature and laser intensity. The higher negative value of $S_{P}$ compared to $S_{A P}$, gives always a larger negative voltage in the parallel magnetization alignment $U_{P}$ than in the antiparallel $U_{A P}$. The experimental data does not match this linear dependence, however, the trend is clearly the same as in the theoretical data: $U_{P}$ has 


\subsection{Experimental Results}

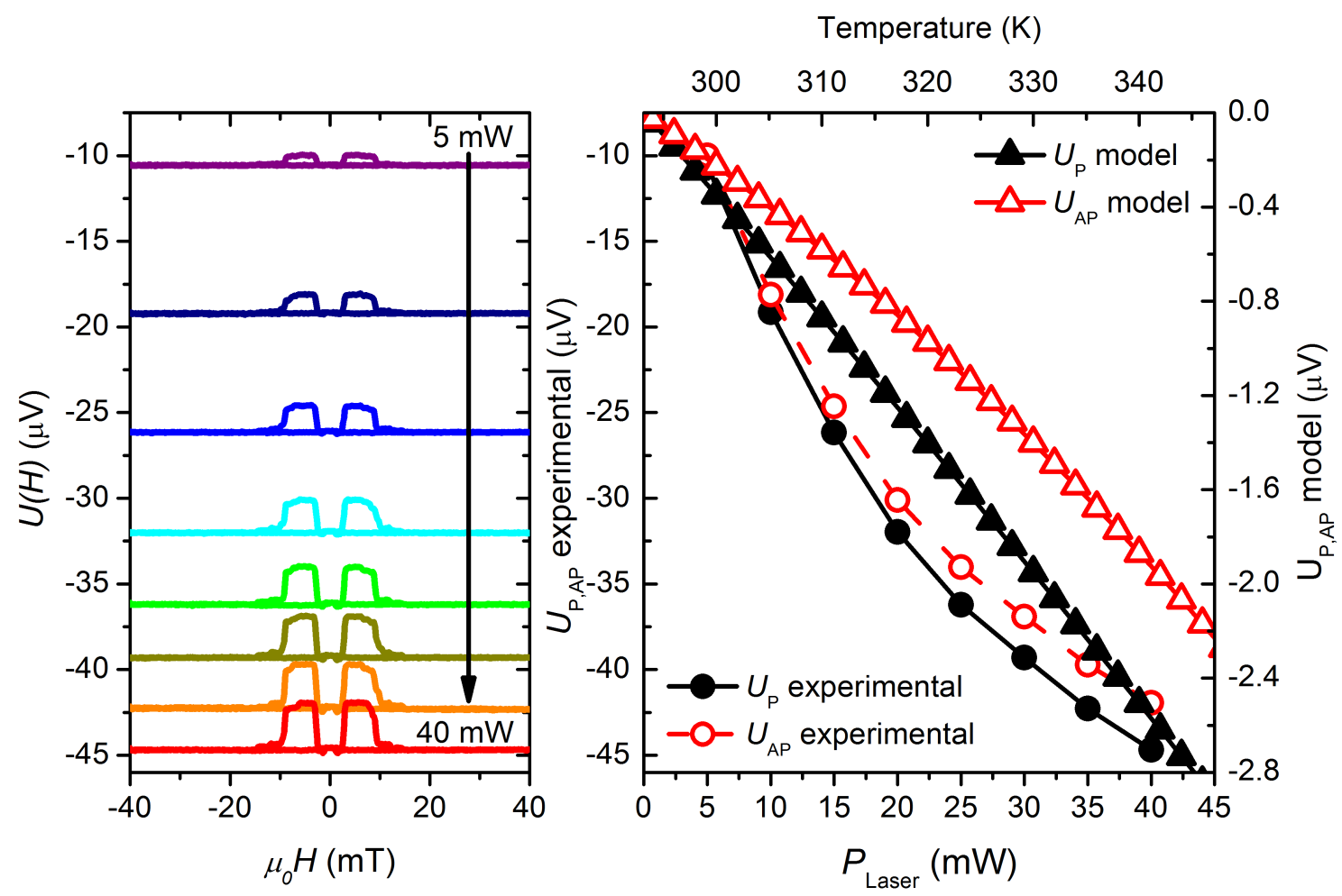

Figure 2.23: Magneto-Seebeck $U(H)$ curves measured on a $1 \times 1 \mu \mathrm{m}^{2}$ tunnel junction using a cw diode laser for heating, with intensities from $P_{\text {Laser }}=$ $5 \mathrm{~mW}-40 \mathrm{~mW}$ (left graph). The right graph shows the voltage in the parallel $U_{P}$ (black) and antiparallel $U_{A P}$ (red) magnetization alignment. The experimental data (circles) is compared to theoretical calculations (triangles). The corresponding temperatures and temperature gradients are taken from COMSOL simulations. The temperature gradients ranging from $\Delta T=8.76 \mu \mathrm{V} / \mathrm{K}$ to $\Delta T=70.5 .1 \mu \mathrm{V} / \mathrm{K}$ were used to calculate the theoretical voltages.

always a larger negative value than $U_{A P}$. The deviation from a linear relation stems, as also in the data presented in figures 2.14 and 2.15(a) from larger temperature gradients aside from the one at the junction, supplying a larger contribution to the measured signal, which are not considered in the theoretical model.

The experimental data shows voltages ranging from $U_{P}=-10.6 \mu \mathrm{V}$ to $U_{P}=$ $-44.6 \mu \mathrm{V}$. This is a deviation of factor 40 and 20 , respectively, from the theoretically predicted values. The voltages in the antiparallel alignment range from $U_{A P}=$ $-10.0 \mu \mathrm{V}$ to $U_{A P}=-41.9 \mu \mathrm{V}$. This gives deviation factors of 50 and 25 , respectively.

Figure 2.25(a) shows the difference of both voltages $\left|U_{P}-U_{A P}\right|$, for the experimental data and the prediction by the theoretical model. The difference extracted from the experimental data is approximately five times higher than the difference form the data calculated by the theoretical model using the transmission function $T_{t}(E)$. Inspite of that, the resulting relative magneto-Seebeck ratio $S_{M S}$ is between $-5.6 \%$ 


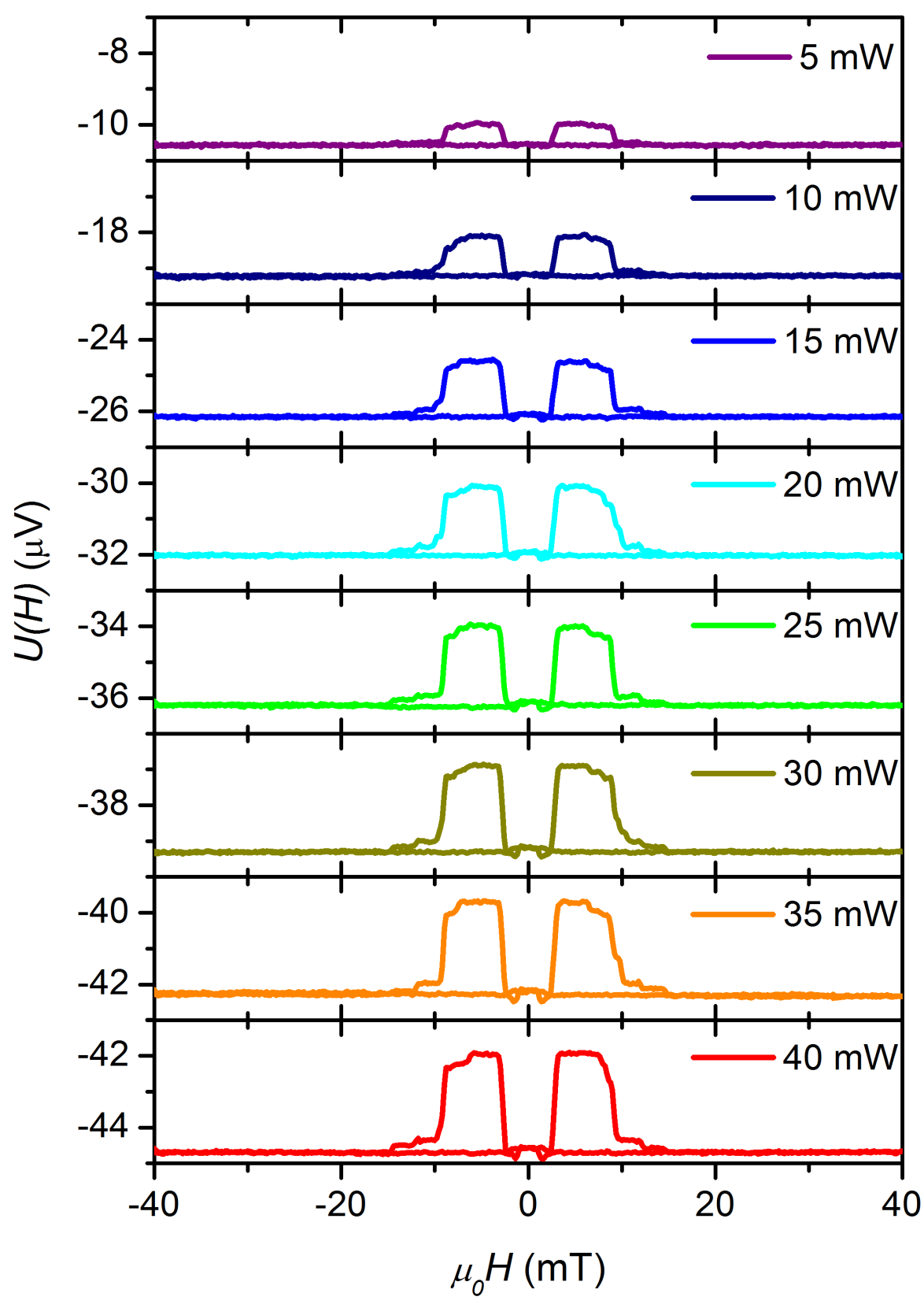

Figure 2.24: Magneto-Seebeck measurements, the same $U(H)$ curves as in figure 2.23 left, plotted in more detail. The curves show that the absolute value of the difference $S_{P}-S_{A P}$ increases with heating laser intensity. The magnetoSeebeck effect is clearly visible in the separation of the parallel and antiparallel magnetization alignment in the region $\mu_{0} H=2 \mathrm{mT}-10 \mathrm{mT}$. 


\subsection{Experimental Results}

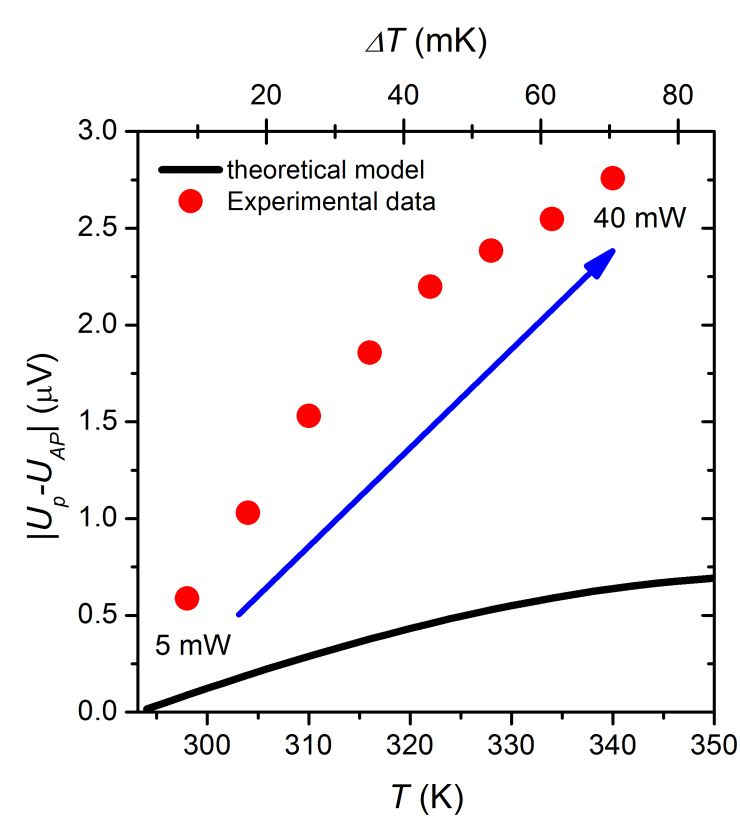

(a) $\left|U_{P}-U_{A P}\right|$ from experimental data and theoretical model.

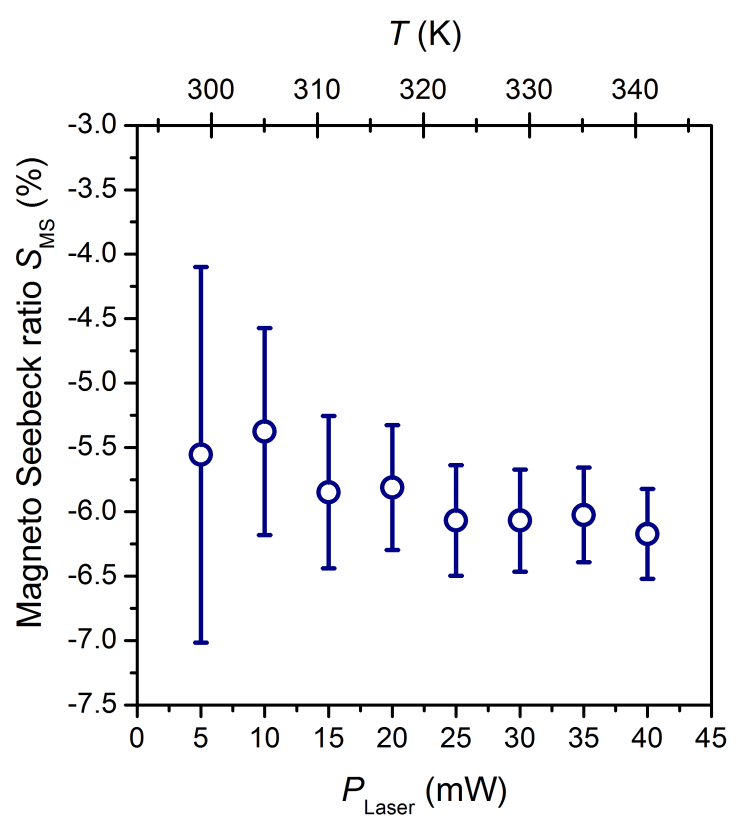

(b) Magneto-Seebeck ratio $S_{M S}$ calculated from the experimental data

Figure 2.25: (a) $\left|U_{P}-U_{A P}\right|$ extracted from experimental data and from the theoretical model for comparison, plotted against the temperature and temperature gradient calculated in COMSOL simulations. (b) Magneto-Seebeck ratio $S_{M S}$ calculated from the same experimental data plotted against the laser intensity $P_{\text {Laser }}$ and the corresponding temperature.

and $-6.2 \%$, roughly one order of magnitude smaller than the theoretically predicted values. The absolute value of $S_{M S}$ is slightly increasing with the temperature (see figure 2.25(b)) in contrast to the trend predicted by theory.

The high voltages in the experimental data compared to the theoretical values, at first view suggest a background comparable to that in the above presented data recorded before the setup has been optimized. At this stage, the background from inhomogeneous heating or laser instabilities can be excluded. Also the contribution from additional temperature gradients at the interfaces of the metallic layers on top of the junction contributing to the signal can not be excluded, but are not significant, because calculating the Seebeck coefficients from the experimental voltages on the basis of the temperature gradients obtained from COMSOL simulations, leads to Seebeck coefficients at 296.1 K: $S_{P}=-1210 \mu \mathrm{V} / \mathrm{K}$ and $S_{A P}=$ $-1140 \mu \mathrm{V} / \mathrm{K}$. At $340 \mathrm{~K}$ both Seebeck coefficients are smaller $S_{P}=-640 \mu \mathrm{V} / \mathrm{K}$ and $S_{A P}=-600 \mu \mathrm{V} / \mathrm{K}$. The absolute values of those Seebeck coefficients are unreasonably high, too high to explain the contribution from the metallic layers on top of the tunnel junction. Besides that, the large difference of the voltages in the parallel and antiparallel magnetization alignment $\left|U_{P}-U_{A P}\right|$ shows that the voltage is mainly generated by the temperature gradient at the $\mathrm{MgO}$ layer. That leaves two 
sources for the rather high Seebeck coefficients and the small Seebeck ratio in the experimental data. First, as discussed above, the Seebeck coefficients are sensitive to the Co and Fe distribution in the bcc structure at the interface to the $\mathrm{MgO}$ layer. Comparing the simulated Seebeck coefficients and the Seebeck ratios to the experimental data in the measured temperature range, both show the same trends. This suggests a dominating alternating-termination structure at the $\mathrm{MgO}$ interface, but in general the structure of the junction differs from the theoretically assumed clean Fe and Co distribution. Second, there still may be discrepancies in the simulated temperatures and temperature gradients, from the actual temperatures in the junction, because all parameters included in the simulation are literature values. At this point, more experimental input into the simulations obtained on the junctions, e.g. reflectivity experiments, to determine the temperature of the laser heated surface are, are needed.

The magneto-Seebeck ratios given in table 2.1. calculated for different interface structures at $300 \mathrm{~K}$, show that the alternating-termination structure has the lowest absolute value and the only negative magneto-Seebeck ratio. That means, a deviation from this structure in the sample, e.g. a mixture of the alternating-termination structure and the Co-termination structure would lead to smaller differences between $S_{P}$ and $S_{A P}$ and thus to a smaller magneto-Seebeck ratio. Additionally, the theoretical Seebeck coefficients are calculated for perfect structures, not taking into account thermal effect contributions from other interfaces in the stack or background noise, which cannot be entirely excluded in the experimental data. Further, the effects at the interfaces between the metallic layers are not considered in the COMSOL simulations. These effects cause voltages which do not depend on the magnetization and therefore decrease the measured magneto-Seebeck ratio.

\section{Temperature Dependent Experiments using the fs Laser}

Further experiments on the same junction R07E02 from sample vz101007b, using a more powerful heat source reveal more features predicted by the calculations based on the theoretical model described in section 2.4.3. These theoretical data in figure 2.20 show that for the alternating-termination structure at the $\mathrm{MgO}$ interface, the magneto-Seebeck ratio changes sign at $T=417 \mathrm{~K}$, because there the Seebeck coefficients $S_{P}$ and $S_{A P}$ intersect, and their ratio inverses. Heating the junction with a fs pulsed laser increases the temperature in the $\mathrm{MgO}$ layer beyond the region, where the sign of the magneto-Seebeck ratio $S_{M S}$ reverses. The experimental equipment, a Ti:Sapphire laser with a regenerative amplifier, ensures energies higher than $0.6 \mu \mathrm{J} / \mathrm{Pulse}$ with a repetition rate of $250 \mathrm{kHz}$, or $1000 \mathrm{~mJ} / \mathrm{cm}^{2}$ that can be deposited at the sample surface.

The measured $U(H)$ curves in figure 2.26 give experimental proof for the reversal of the ratio between the voltage in the parallel and the antiparallel magnetization alignment $\left(U_{P}\right.$ and $\left.U_{A P}\right)$, respectively. This reversal occurs with increasing laser intensity for high enough heating fluences above $600 \mathrm{~mJ} / \mathrm{cm}^{2}$. The voltage in the par- 


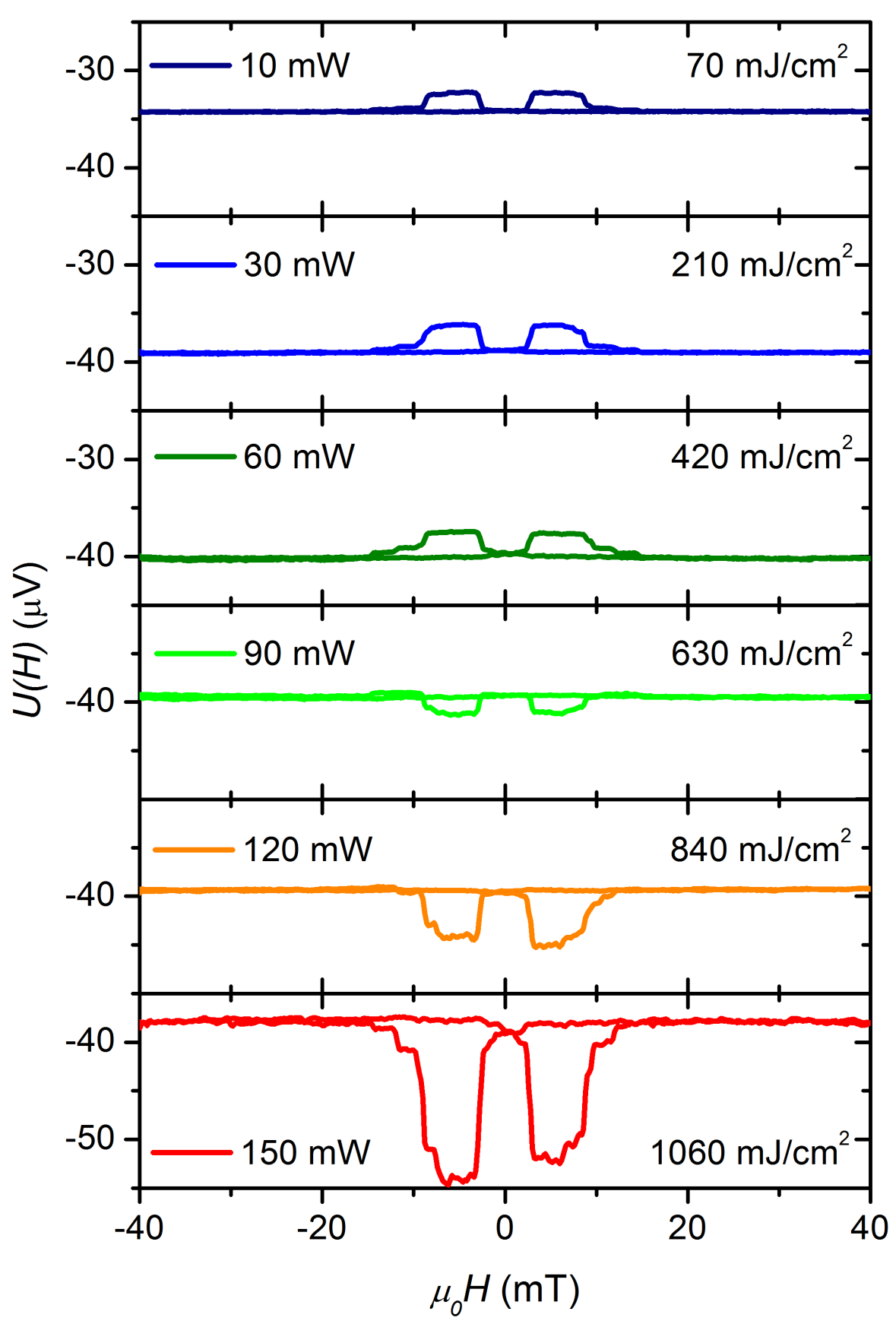

Figure 2.26: $U(H)$ curves measured using high intensities of a pulsed Ti:Sapphire laser, reaching heating fluences above $1060 \mathrm{~mJ} / \mathrm{cm}^{2}$, confirm the theoretical prediction in a real tunnel junction. At heating fluences above $600 \mathrm{~mJ} / \mathrm{cm}^{2}$ the ratio between $U_{P}$ and $U_{A P}$ inverses. 


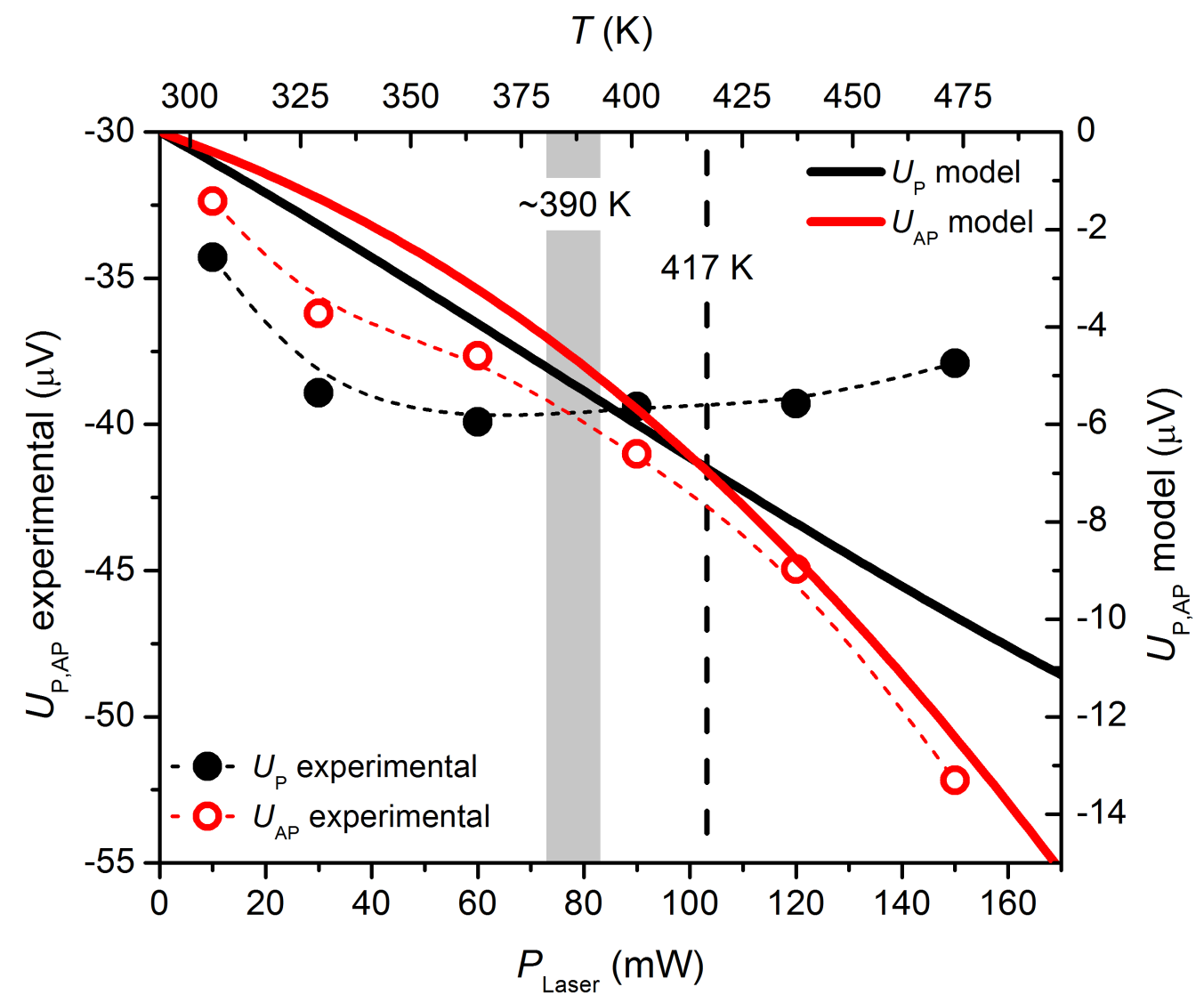

Figure 2.27: Experimentally measured $U_{P}$ (black dots) and $U_{A P}$ (red circles), plotted against the laser intensity $P_{\text {Laser }}$ (left and bottom scale, the short dashed lines are guides to the eye), and the theoretically calculated $U_{P}$ (black line) and $U_{A P}$ (red line) plotted against the temperature (right, top scale). The gray pane and the dashed line indicate the temperatures and corresponding pump fluences at which the ratio between $U_{P}$ and $U_{A P}$ reverses in the experimental and theoretical data, respectively.

allel magnetization alignment $U_{P}$ remains constantly at around $-35 \mu \mathrm{V}$ to $-40 \mu \mathrm{V}$ throughout the whole dataset, while the voltage in the antiparallel magnetization alignment increases its absolute value from $-55 \mu \mathrm{V}$ to $-35 \mu \mathrm{V}$. At the same time, the difference between $U_{P}$ and $U_{A P}$ increases constantly with increasing temperature, except for the laser power densities around which the ratio between $U_{P}$ and $U_{A P}$ reverses. The temperature dependent difference of both voltages $\left|U_{P}-U_{A P}\right|$ shows a temperature dependence of the magneto-Seebeck effect and together with the reversal of the ratio between $U_{P}$ and $U_{A P}$ similarities to the data calculated using the theoretical model, presented in figure 2.20 .

Figure 2.27 shows voltages in the parallel and antiparallel magnetization alignment $U_{P}$ and $U_{A P}$ (left y-axis), extracted from the $U(H)$ curves presented in figure 2.26. They are plotted against the laser intensity as black dots and red circles, re- 


\subsection{Experimental Results}

spectively (bottom x-axis, the dashed lines are guides to the eye). The same plot includes for comparison the Seebeck voltages in the parallel $U_{P}$ and antiparallel $U_{A P}$ magnetization alignment calculated from the theoretical Seebeck coefficients $S_{P}$ and $S_{A P}$ displayed in figure 2.20 and temperature gradients to the corresponding temperatures extracted from COMSOL simulations given in figure 2.11. The theoretical voltages are represented by the black and red line, respectively (right y-axis) and are plotted in the temperature range from $293.15 \mathrm{~K}$ to $550 \mathrm{~K}$ (top x-axis). The comparison is carried out for the Seebeck voltages $U_{P, A P}$ rather than the Seebeck coefficients $S_{P, A P}$, because as discussed above, the extraction of the Seebeck coefficients from the experimental data leads to exaggerated values, which can not be verified.

The temperature scale (top x-axis) is determined from the relation for the temperature and temperature gradients extracted from COMSOL simulations for temperature distribution in laser heated tunnel junctions in [69] and plotted in figure 2.11. From this data, the following relations between the temperature, the temperature gradients and the laser intensity is derived:

$$
\begin{aligned}
\Delta T & =0.0018 \frac{\mathrm{K}}{\mathrm{mW}} \cdot P_{\text {Laser }} \\
T_{\mathrm{MgO}} & =1.2 \frac{\mathrm{K}}{\mathrm{mW}} \cdot P_{\text {Laser }}+293.15 \mathrm{~K}
\end{aligned}
$$

Equations 2.36 are also used to calculate the theoretical voltages $U_{P}$ and $U_{A P}$ from the Seebeck coefficients for given temperatures. They determine the temperature difference across the $\mathrm{MgO}$ layer $\Delta T$. Using equations 2.36 to calibrate the temperature axis to the laser intensity axis shows a discrepancy by approximately $20 \mathrm{~mW}$ or $25 \mathrm{~K}$ between the points at which the Seebeck coefficients intersect. In the theoretical data this point is at the temperature of $417 \mathrm{~K}$, which according to the calibration is at around $P_{\text {Laser }}=100 \mathrm{~mW}$, indicated by the dashed black line in figure 2.27. At these heating fluences the predicted temperature gradient is around $\Delta T=180 \mathrm{mK}$. Whereas the ratio between both $U_{P}$ and $U_{A P}$ in the experimental data reverses at around $P_{\text {Laser }}=80 \mathrm{~mW}$, which corresponds to a temperature around $390 \mathrm{~K}$ indicated by the gray pane in figure 2.27. Further, the data shows an offset between the experimentally measured and theoretically calculated voltage. At low laser intensities $P_{\text {Laser }}<60 \mathrm{~mW}$ and therefore low temperatures above room temperature and small temperature gradients, the theoretical data shows small voltages up to $-4 \mu \mathrm{V}$. While the experimentally obtained voltages are between $-30 \mu \mathrm{V}$ and $-40 \mu \mathrm{V}$. This offset arises partially due to temperature gradients occurring at the interfaces of the other layers in the stack on top of the tunnel junction and due to the distribution of the $\mathrm{Co}$ and $\mathrm{Fe}$ atoms in the bcc structure at the interface to the $\mathrm{MgO}$ tunnel barrier, which is not a perfect alternating-termination structure. Besides the offset in the generated voltage, both, the theoretically determined and the experimentally measured voltage in the antiparallel magnetization alignment $U_{A P}$ show the same trend, a decreasing voltage with increasing temperature and temperature gradient. In contrast to that, the voltages in the parallel magnetization alignment $U_{P}$ do not follow the same trend, while the experimentally determined $U_{P}$ remains at 


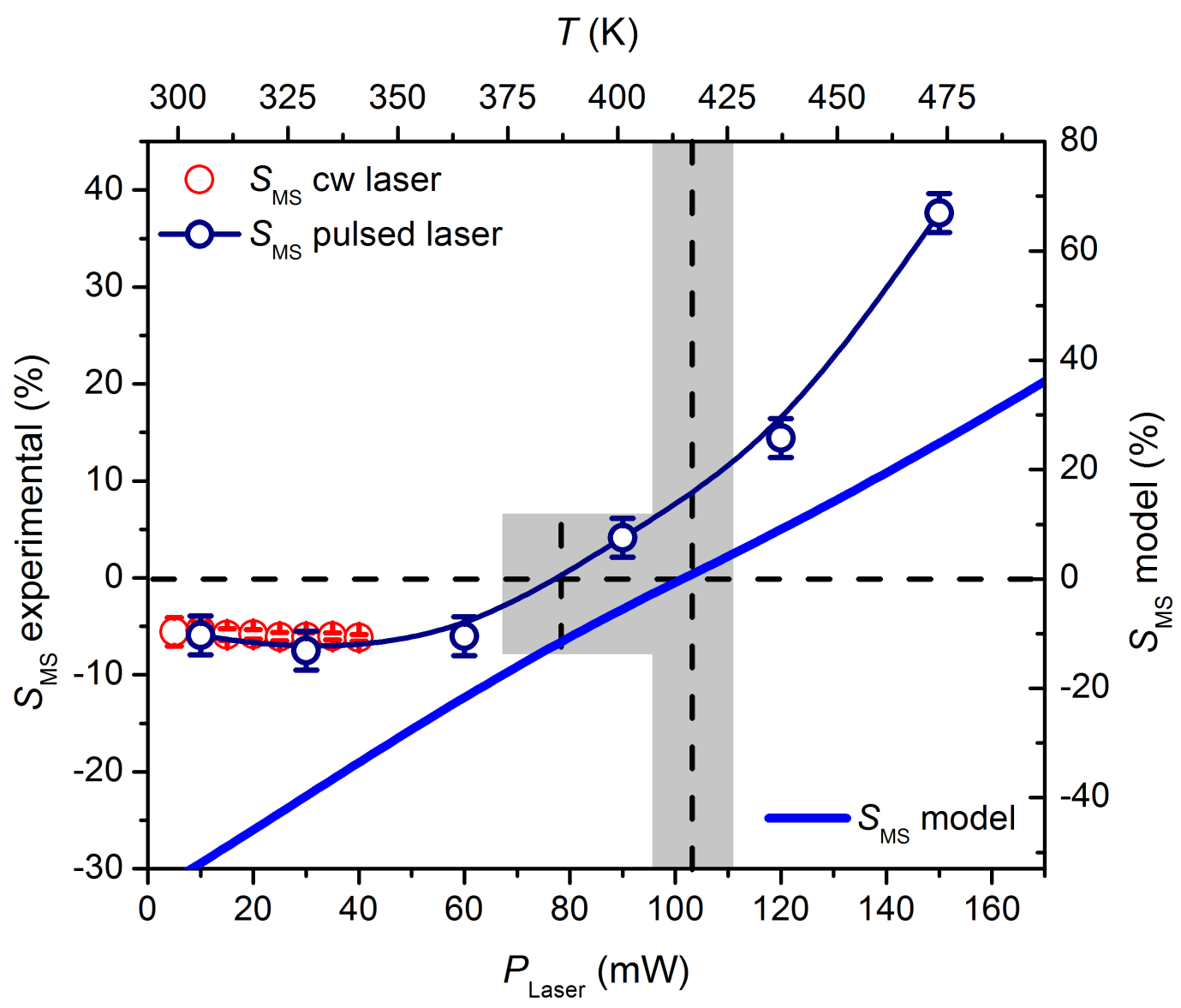

Figure 2.28: The experimentally (dark blue and red circles, left scale) and theoretically (light blue line, right scale) determined magneto-Seebeck ratio $S_{M S}$, plotted against the laser intensity (bottom x-axis) and the temperature (top x-axis), respectively. The dark blue line is a guide to the eye. The gray pane and black dashed lines indicate the points where $S_{M S}$ changes sign.

a rather constant value around $-40 \mu \mathrm{V}$, the theoretically determined $U_{P}$ increases with increasing temperature gradient. In conclusion, figure 2.27 shows a fairly good agreement between the theoretical prediction and the experimental data, taking into account, that the theoretical calculations are performed on perfect crystalline junctions with a perfect Co and Fe distribution, which is not known for the samples. As shown above, the Co and Fe distribution or change in concentration can change the Seebeck coefficients substantially, leading to disagreement between theory and experiment. Further, the access to temperature inside of the junctions is not accessible experimentally, only simulations are available so far, therefore a discrepancy of $20 \mu \mathrm{V}$ is rather small. Finally, the contribution of the gradients at the other layer interfaces also contributes to the measured voltage and thus to the offset in the data, but is not considered as significant in the simulated data.

In figure 2.28, the magneto-Seebeck ratio $S_{M S}$ obtained from the experimental 


\subsection{Experimental Results}

data is compared to the ratio obtained from the theoretical calculations. The temperature is calibrated to the laser intensity using the same procedure as in figure 2.27. This leads to the same discrepancy between the experimental and theoretical data at the point, where $S_{M S}$ changes sign, as where $U_{P}$ and $U_{A P}$ intersect. On behalf of a complete picture, also the $S_{M S}$ values obtained, from the experimental data recorded using the $\mathrm{cw}$ diode laser is plotted. Because the magneto-Seebeck ratio is calculated from the voltages presented in figure 2.27, the features observed there are mirrored by the $S_{M S}$ values. The comparatively small difference between both voltages $\left|U_{P}-U_{A P}\right|$ in the experimental data measured at lower laser intensities, considering the offset voltage and compared to $\left|U_{P}-U_{A P}\right|$ for the theoretical data at low temperatures above room temperature, results in an experimental $S_{M S}$ which is up to six times smaller than the theoretical one. However, $\left|U_{P}-U_{A P}\right|$ measured at low laser intensities shows values in the same range, as those measured in other samples at the same laser intensities and mirrors the same magnitude of the magneto-Seebeck effect. Whereas, at higher temperatures, after the sign of $S_{M S}$ is reversed to a positive value, the experimental magneto-Seebeck ratio is slightly larger than the theoretical one, because there $\left|U_{P}-U_{A P}\right|$ is bigger due to the rather constant value of $U_{P}$ in the whole temperature range. So that the deviations between the experimental data and the theoretical predictions have the same origins as the deviations in the Seebeck voltages discussed above.

\section{Autocorrelation Experiments}

An extension of the experimental setup by implementing a delay stage allows to measure the voltage generated at the tunnel junction by delaying the arrival of two heating pulses at the sample surface. The laser beam is divided by a beam splitter into two beams. One beam, with one half of the intensity, is directed to the mechanical delay stage which, by changing the path length of the beam, can delay the time of arrival at the sample surface with a temporal resolution of $\Delta \tau=20 \mathrm{fs}$. The other beam is directed straight to the sample. Both beams merge before passing the objective in front of the sample. The setup is still functioning as depicted in figure 2.8, with the additional possibility of pulse delay. At 0 ps delay, both laser pulses arrive at the sample surface simultaneously, acting a single pulse which contains the initial energy, before the splitting. The voltage changes nonlinearly with the laser intensity, because the Seebeck coefficients are nonlinear. Therefore, a nonlinear increase in the voltage across the junction is expected, when their time of arrival at the sample surface is delayed. This experimental alignment corresponds to a standard autocorrelation experiment. From the decay time of the voltage, the decay times of the temperatures generated inside the junction can be concluded. Figure 2.29 shows the autocorrelation voltage signal of the examined tunnel junction R07E02. There are two relaxation times clearly visible and determined via a double exponential fit to the data. The first very short one $t_{1}=184 \mathrm{fs}$ stems from hot electron excitations. The second, slower relaxation time $t_{2}=88.7 \mathrm{ps}$ is consistent with the 


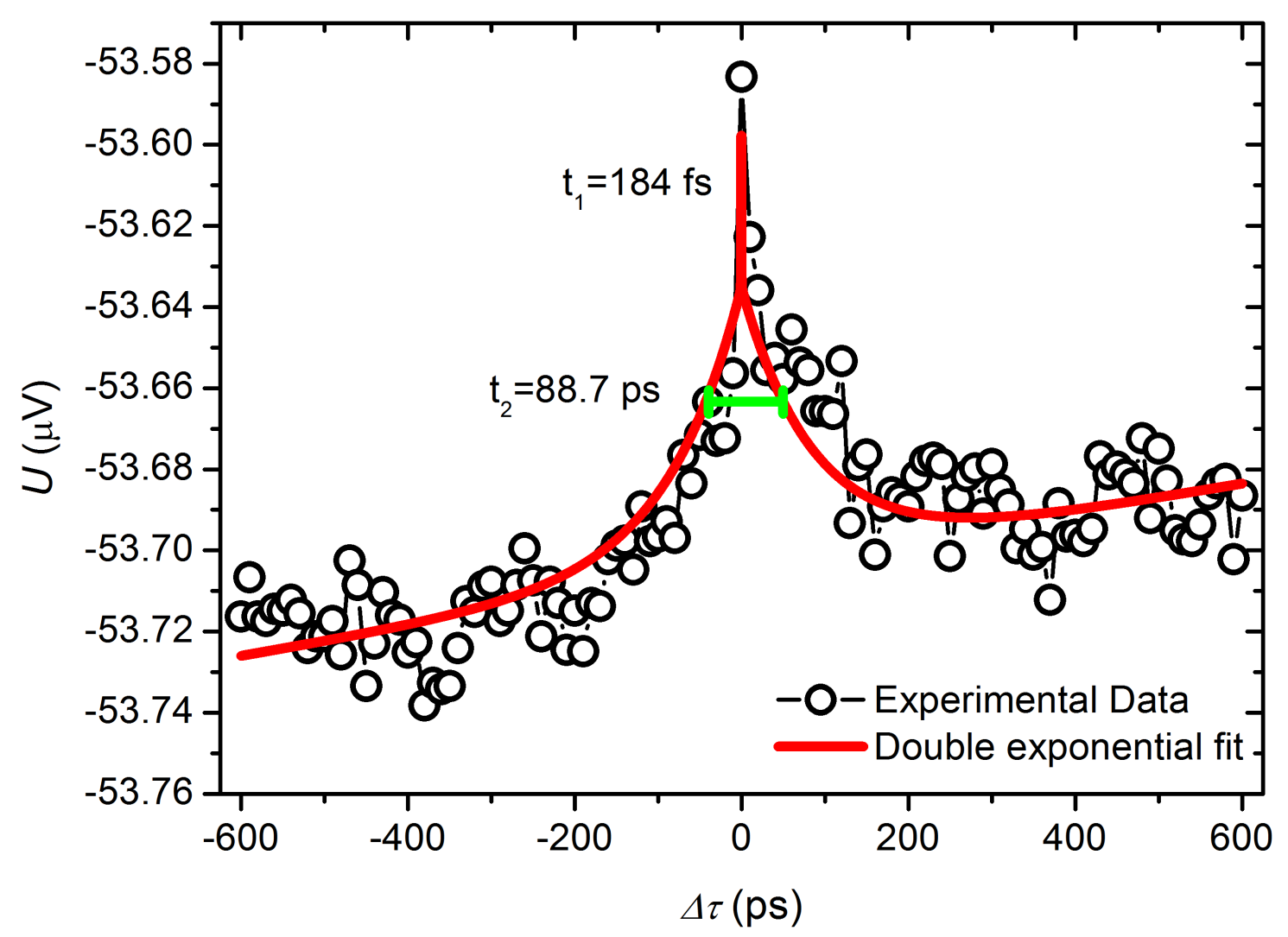

Figure 2.29: Autocorrelation voltage signal of the tunnel junction, and the decay times, extracted from the double exponential fit to the experimental data.

times determined in COMSOL simulations given in [69]. There, temperatures up to $800 \mathrm{~K}$ are achieved, but decay to below $400 \mathrm{~K}$ after $100 \mathrm{ps}$ and remain at this elevated temperature for up to $1 \mathrm{~ns}$. That means, the laser pulses generate high temperatures across the tunnel junction, however, their decay is very fast, leaving rather low temperatures in the stationary cases. Therefore, the temperature gradients in the stationary case are also lower, because at the same time, at which the high temperature decreases, the surroundings temperatures rise, leaving smaller temperature differences. 


\subsection{Concluding Remarks}

\subsection{Concluding Remarks}

\subsubsection{Summary}

The goal in the second part of this thesis has been to set up an experiment and measure the magneto-Seebeck effect in magnetic tunnel junctions. The result is a setup in which specific MTJs situated in an external magnetic field can be heated optically by a laser beam of requested intensity. Different lasers can be coupled into the experiment and exchanged quickly with the help of preset mirrors and pinholes. The voltage generated by the temperature gradient originating from the heating of the top layer by the laser beam is measured and recorded by the lock-in technique. A large part of the experimental work has been devoted to the optimization of this setup from the first measured curve, in which the magneto-Seebeck effect can be distinguished (figure 2.13) and which contains a high level of noise, to the temperature dependent measurements with a cw diode laser and the femtosecond pulsed laser (figure 2.26), where the noise is largely eliminated. The setup has been improved by stabilizing the intensity of the light source, by eliminating possible electric currents due to additionally increased impedance of the lock-in amplifier and by determining the right modulation frequency. Further unwanted effects could be minimized by choosing small MTJs and ensuring homogeneous heating.

Several features predicted by ab-initio calculations based on the energy dependent transmission function for the transmission probability of electrons through the $\mathrm{MgO}$ tunnel barrier have been verified in the experiments. Recording the temporal traces of the signal arriving at the lock-in amplifier input enables the determination and clarification of the sign of the voltage generated at the $\mathrm{MgO}$ layer in a MTJ. With this procedure the negative Seebeck coefficients predicted by the theoretical model can be distinguished in the experimental data. Measurements at higher intensities even reveal the change of sign in the magneto-Seebeck ratio predicted in the theoretical calculations. There is only one a small deviation of approximately $20 \mathrm{~K}$ in the temperature calibration calculated from the applied laser intensity. The offset in the measured voltage is ascribed to voltages generated by temperature gradients at other interfaces of the layer stack, than at the $\mathrm{MgO}$ barrier.

The extension of the setup to measure the autocorrelation of the voltage signal, reveals timescales at which the voltages are generated identical to those obtained from simulations. These timescales will help to further improve the modulation technique.

\subsubsection{Outlook}

The experimental setup introduced and discussed above is technically mature to deliver further experimental data for the analysis of thermo-magnetic effects and gain a deeper understanding of the underlaying mechanisms. The experiments can be easily expanded to measure thermally assisted spin transfer torque, as soon as $\mathrm{MgO}$ based tunnel junctions with 3 monolayer thick barriers can be produced reliably 
[34]. In contrast to conventional spin-electronic devices, further size reduction in thermally driven 'spincaloritronic' devices will increase the observed effects, because electric current densities are not significant for their functionality.

However, there is still potential to further improve the setup and gain more reliable experimental data without the previously discussed voltage offset.

The first major challenge is the access to precise temperatures and temperature gradients across the $\mathrm{MgO}$ tunnel barrier. COMSOL simulations indicate the correct order of magnitude [64, 69], but in order to obtain more reasonable results, experimentally obtained input parameters for the simulations are necessary. For this purpose additional experiments allowing to infer the surface temperatures of the irradiated layers are possible. The two temperature model used in [6] and in chapter 1 proves to be the right tool for this assignment. Performing these timeresolved reflectivity experiments to the Au contact layers of the junctions, will allow the determination of the surface temperature and its temporal evolution, providing information about the magnitude of the peak temperature and the time it needs to relax to the equilibrium state. Probing the time-resolved reflectivity at positions different from the location of heating will provide additional information about the temperature distribution on the surface. This experimentally obtained information about the behavior of the surface temperatures might be used as input for temperature simulations and improve the predictions. More precise knowledge of the heat distribution at the surface of the Au contact pad will help to quantify the amount of heat distributed along the direction of the junction's thickness. Besides the role that the junction size plays in the heat distribution should be estimated by computing heat distributions, and thus the temperatures and temperature gradients for different junction sizes.

The second opportunity for a more reliable access to temperature is by simplifying the layer stack of the junction. Decreasing junction sizes to the nano scale will diminish the temperature inhomogeneities in the areas of the layers. By additionally reducing the number and the thickness of layers on top of the junctions, keeping in mind that the junctions need to maintain a high resistivity and TMR ratio, the temperature distribution throughout the junction will be improved and voltages generated in those layers will be reduced. Besides that, the loss of heat conducted through the top layers will be decreased and higher temperatures at lower laser intensities will be possible, expanding the experimentally accessible temperature range. 



\section{A Calculating the per Layer Absorbed Power Density}

The absorbed power density $P\left(z=d_{\mathrm{Ni}}\right)$ deposited at the nickel surface by laser pulses with the fluence $F$ in [6] is calculated according to the Lambert-Beer law:

$$
P(\vec{r})=\frac{\alpha_{a b s} \cdot F(\vec{r}, t) \cdot e^{-z / \Lambda_{\mathrm{opt}}}}{\Lambda_{\mathrm{opt}} \cdot\left(1-e^{-d / \Lambda_{\mathrm{opt}}}\right)} \equiv \alpha_{a b s} \cdot F(\vec{r}, t) \cdot g(z)
$$

where $\vec{r}=(x, y, z)$ can be considered in one dimension, taking into account only the $z$ direction, because only the heat evolution along the penetration depth is investigated. The absorption coefficient $\alpha_{a b s}$ is defined in section 1.4 .2 , and $\Lambda_{\mathrm{opt}}=$ $14.5 \mathrm{~nm}$ for nickel.

For nickel films thinner than the optical penetration depth, $d_{\mathrm{Ni}}<\Lambda_{\mathrm{opt}}$, the factor $\exp \left(-z / \Lambda_{\text {opt }}\right)$ can be substituted by the average over the layers in a film of the thickness $d_{\mathrm{Ni}}$ :

$$
\begin{aligned}
\left\langle\exp \left(-z / \Lambda_{\mathrm{opt}}\right)\right\rangle_{d_{\mathrm{Ni}}} & =\frac{1}{d_{\mathrm{Ni}}} \int_{0}^{d_{\mathrm{Ni}}} \exp \left(-z / \Lambda_{\mathrm{opt}}\right) d z=\frac{1-\exp \left(-d_{\mathrm{Ni}} / \Lambda_{\mathrm{opt}}\right)}{d_{\mathrm{Ni}} / \Lambda_{\mathrm{opt}}} \\
& \Rightarrow g(z)=1 / d_{\mathrm{Ni}}
\end{aligned}
$$

This formula looks similar to the one defined for the determination of the transmission in section 1.4.2. The difference is that the formula to determine the transmission considers the amount of energy included in the laser pulse being absorbed by the nickel film, whereas this formula determines rather the distribution of the absorbed energy to the different layers. The per layer absorbed power density by a nickel film of thickness $d_{\mathrm{Ni}}<\Lambda_{\mathrm{opt}}, \tilde{P}\left(z=d_{\mathrm{Ni}}\right)$, normalized to the absorbed power density $P_{0}$ by a nickel film $d_{\mathrm{Ni}}^{0}=10 \mathrm{~nm}$ at the pump fluence $F_{0}=10 \mathrm{~mJ} / \mathrm{cm}^{2}$ is given by:

$$
\tilde{P}\left(z=d_{\mathrm{Ni}}\right)=P\left(z=d_{\mathrm{Ni}}\right) / P_{0}=\frac{F}{F_{0}} \frac{d_{\mathrm{Ni}}^{0}}{d_{\mathrm{Ni}}} \cdot \frac{\alpha_{a b s}}{\alpha_{a b s}^{0}} .
$$

With $\alpha_{a b s}^{0}$ being the absorption coefficient determined for the $10 \mathrm{~nm}$ thick nickel film.

In nickel films thicker than the penetration depth $d_{\mathrm{Ni}}>\Lambda_{\mathrm{opt}}$, only the magnetization and reflectivity at the surface, as deep as the optical penetration depth can be monitored using an optical probe. Therefore, the function $g(z) \cong g_{\text {opt }}(z)$ can be considered:

$$
g_{\mathrm{opt}}(z)=\frac{\left\langle\exp \left(-z / \Lambda_{\mathrm{opt}}\right)\right\rangle_{\Lambda_{\mathrm{opt}}}}{\Lambda_{\mathrm{opt}} \cdot\left(1-\exp \left(-d_{\mathrm{Ni}} / \Lambda_{\mathrm{opt}}\right)\right)} .
$$


Using the above expression for $\left\langle\exp \left(-z / \Lambda_{\text {opt }}\right)\right\rangle_{\Lambda_{\mathrm{opt}}}$, equation A.1 with the integral going up to the penetration depth, yields:

$$
g_{\mathrm{opt}}(z)=\frac{(1-\exp (-1))}{\Lambda_{\mathrm{opt}} \cdot\left(1-\exp \left(-d_{\mathrm{Ni}} / \Lambda_{\mathrm{opt}}\right)\right)} .
$$

This gives a per layer absorbed power density normalized to $P_{0}$ :

$$
\tilde{P}\left(z=d_{\mathrm{Ni}}\right)=P\left(z=d_{\mathrm{Ni}}\right) / P_{0}=\frac{F}{F_{0}} \frac{d_{\mathrm{Ni}}^{0}}{\Lambda_{\mathrm{opt}}} \frac{1-\exp (-1)}{1-\exp \left(-d_{\mathrm{Ni}} / \Lambda_{\mathrm{opt}}\right)} \frac{\alpha_{a b s}}{\alpha_{a b s}^{0}} .
$$

The parameters $P / P_{0}$ plotted in figure 1.9 are calculated according to these equations, using $\alpha_{a b s}$ as given in section 1.4.2. 


\section{B TRMOKE and Time-Resolved $\Delta R$ Experimental Data}

The TRMOKE data $\Delta M / M_{300 \mathrm{~K}}(\Delta \tau)$ and the time-resolved change in reflectivity data $\Delta R(\Delta \tau)$, are both plotted as a functions of the time delay $\Delta \tau$. The reflectivity data is scaled to obtain the temperature following the procedure described in section 1.3.3. After determining the temperature at $\Delta \tau=20 \mathrm{ps}$ from the magnetization data, the whole reflectivity datasets are multiplied by the factor $s$, determined from the equation:

$$
T(\Delta \tau=20 \mathrm{ps})[\mathrm{K}]=s[\mathrm{~K} / \mathrm{mV}] \cdot \Delta R(\Delta \tau=20 \mathrm{ps})[\mathrm{mV}]+300 \mathrm{~K} .
$$

This procedure is applied for the data obtained from measurements on the nickel films up to the thickness around $20 \mathrm{~nm}$. Figure B.12 shows only the reflectivity data. After excitation at $\Delta \tau=0 \mathrm{ps}$, the signal rises, but during the relaxation process it becomes negative. The assumption $T_{e} \propto \Delta R$ is not fulfilled anymore. This change of sign stems from two contributions to the reflectivity signal. There is one contribution from the electrons and one from the lattice. For the thinner films, the lattice contribution is small, positive, and after around 5 ps at a constant value, because the change of the lattice temperature is small, compared to the electrons. Therefore the electron contribution is dominating the reflectivity signal. For nickel films thicker than $20 \mathrm{~nm}$ the lattice contribution changes sign, resulting in a negative reflectivity signal, and does not mirror the electron temperature $T_{e}$ anymore [20].

The experimental data are plotted with the color spectrum from blue to red, where blue corresponds to low pump fluences $10 \mathrm{~mJ} / \mathrm{cm}^{2}$ (low color temperature) and red corresponds to $50 \mathrm{~mJ} / \mathrm{cm}^{2}$ (warm color temperature). The TRMOKE data for the $15 \mathrm{~nm}$ nickel film includes fitted function analytically obtained from the $3 \mathrm{~T}$ model taken from [6] (dashed, pink) for comparison. The fits are in agreement with the fits obtained in the present work. 


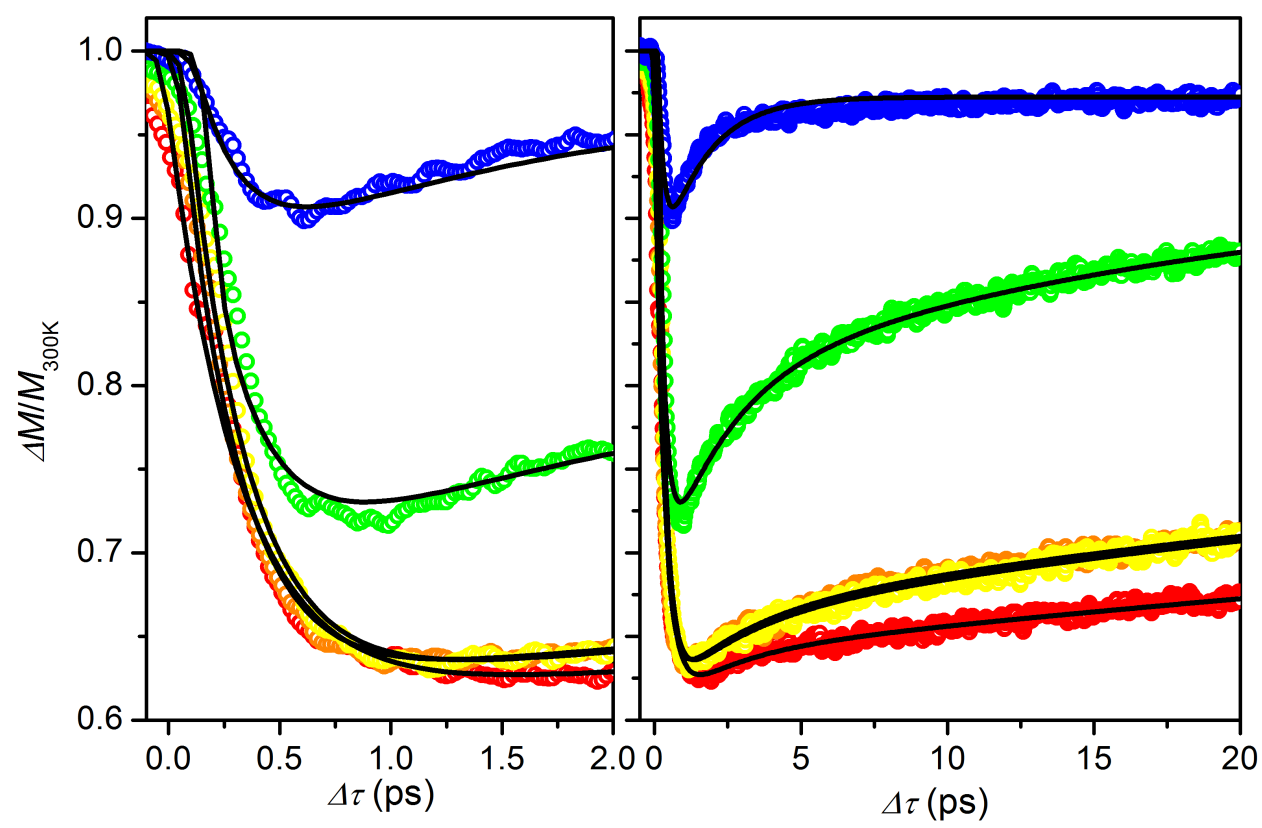

Figure B.1: TRMOKE data rescaled to the demagnetization for the $2 \mathrm{~nm}$ nickel film. Colored - experimental data, black lines are fits of the $3 \mathrm{~T}$ model to the experimental data.

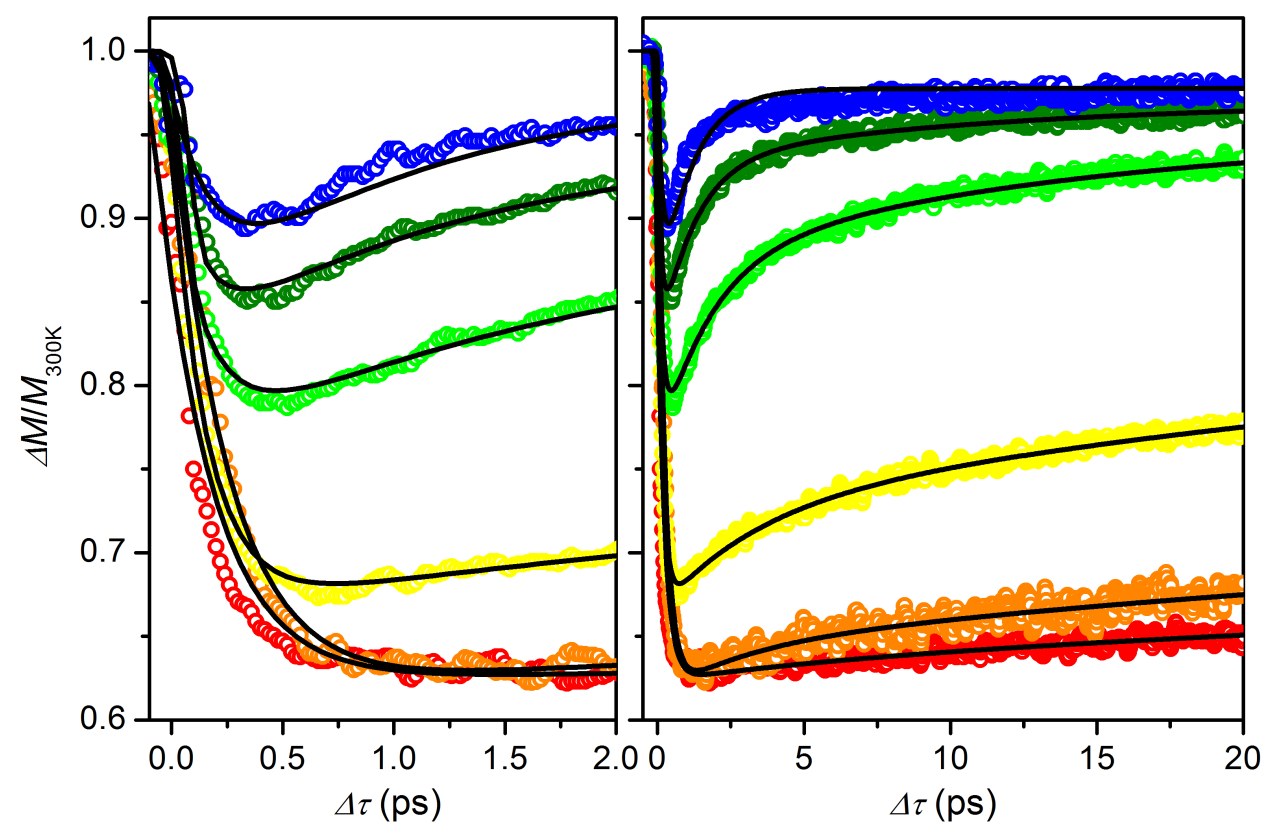

Figure B.2: TRMOKE data rescaled to the demagnetization for the $5 \mathrm{~nm}$ nickel film. Colored - experimental data, black lines are fits of the $3 \mathrm{~T}$ model to the experimental data. 
B TRMOKE and Time-Resolved $\Delta R$ Experimental Data

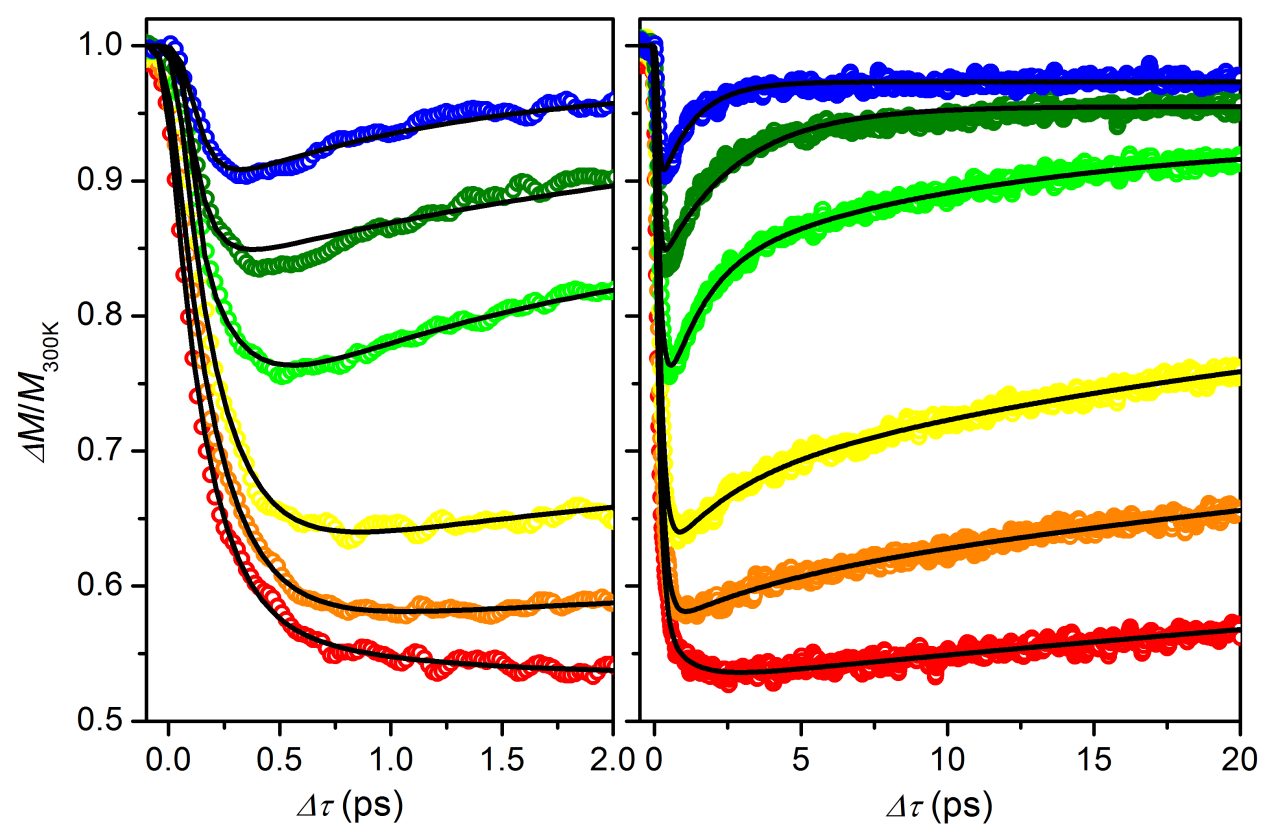

Figure B.3: TRMOKE data rescaled to the demagnetization for the $10 \mathrm{~nm}$ nickel film. Colored - experimental data, black lines are fits of the $3 \mathrm{~T}$ model to the experimental data.

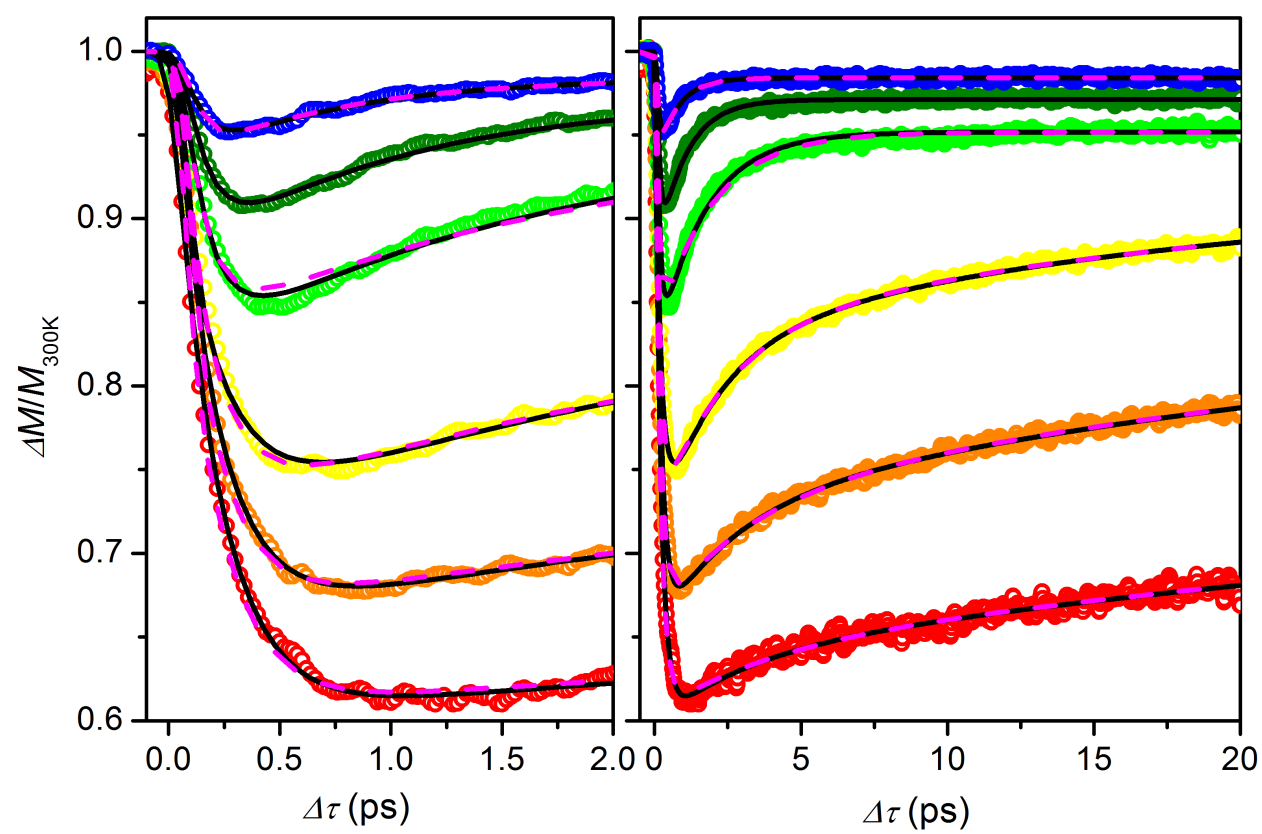

Figure B.4: TRMOKE data rescaled to the demagnetization for the $15 \mathrm{~nm}$ nickel film. Colored - experimental data, black lines are fits of the $3 \mathrm{~T}$ model to the experimental data. 


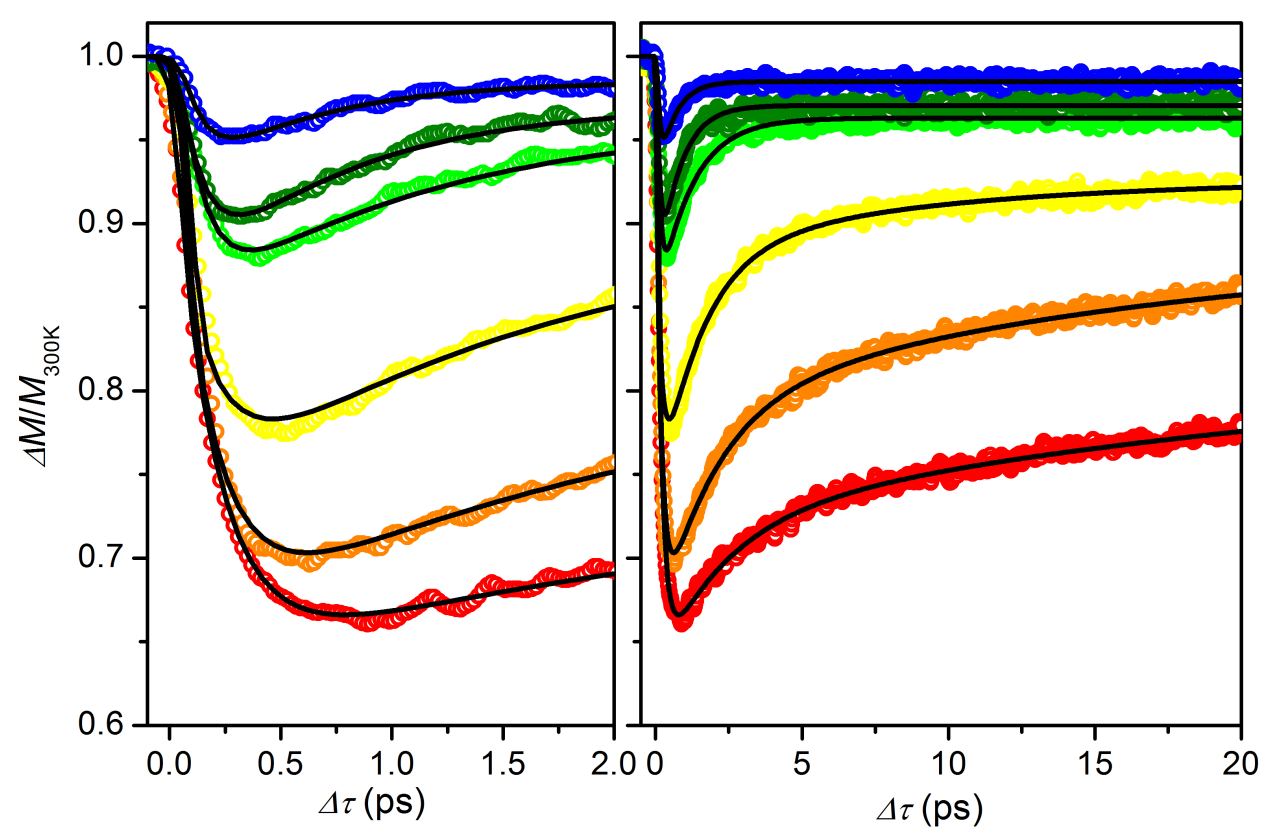

Figure B.5: TRMOKE data rescaled to the demagnetization for the $20 \mathrm{~nm}$ nickel film. Colored - experimental data, black lines are fits of the $3 \mathrm{~T}$ model to the experimental data.

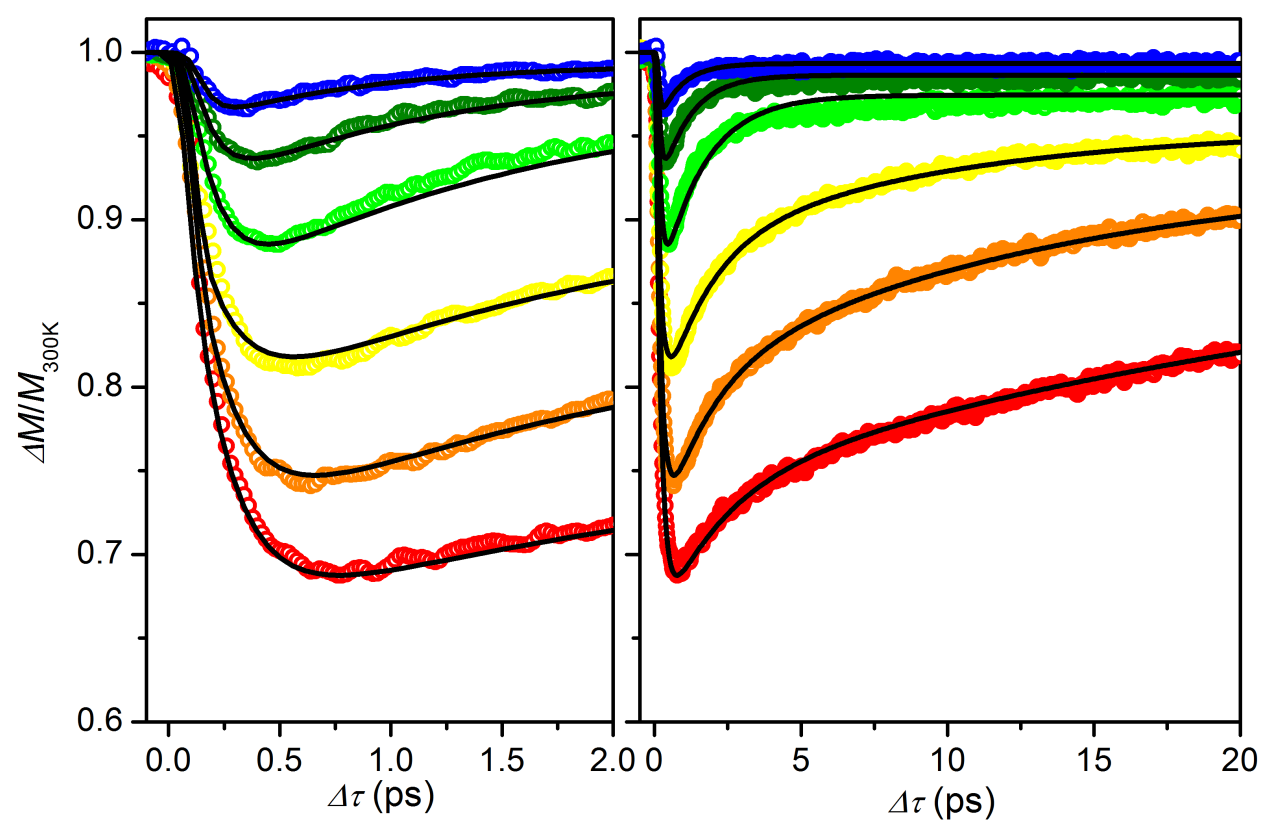

Figure B.6: TRMOKE data rescaled to the demagnetization for the $40 \mathrm{~nm}$ nickel film. Colored - experimental data, black lines are fits of the $3 \mathrm{~T}$ model to the experimental data. 
B TRMOKE and Time-Resolved $\Delta R$ Experimental Data

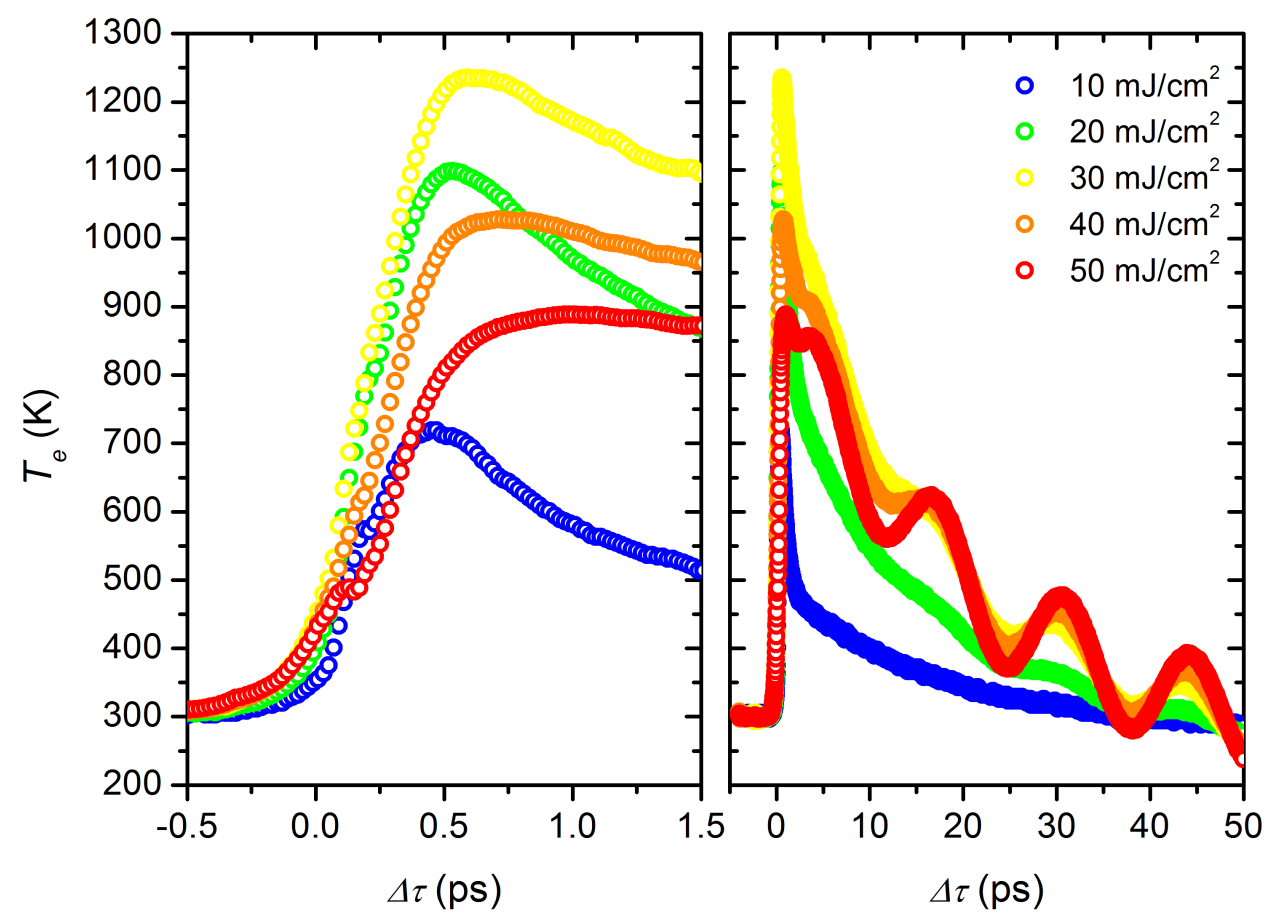

Figure B.7: Time-resolved temperature change in a $2 \mathrm{~nm}$ nickel film. Rescaled from the measured reflectivity data, by the procedure described in section 1.3 .3 .

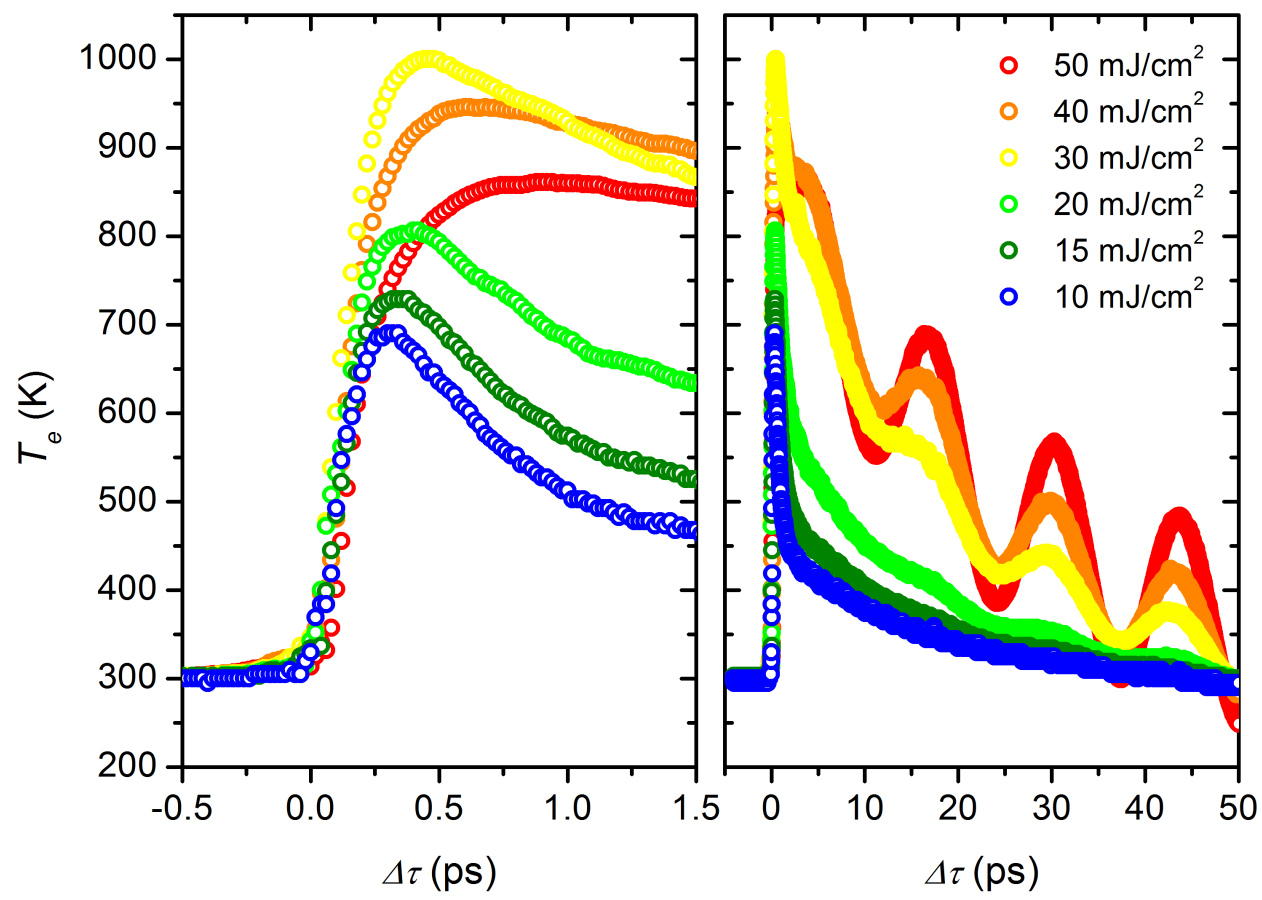

Figure B.8: Time-resolved temperature change in a $5 \mathrm{~nm}$ nickel film. Rescaled from the measured reflectivity data, by the procedure described in section 1.3 .3 


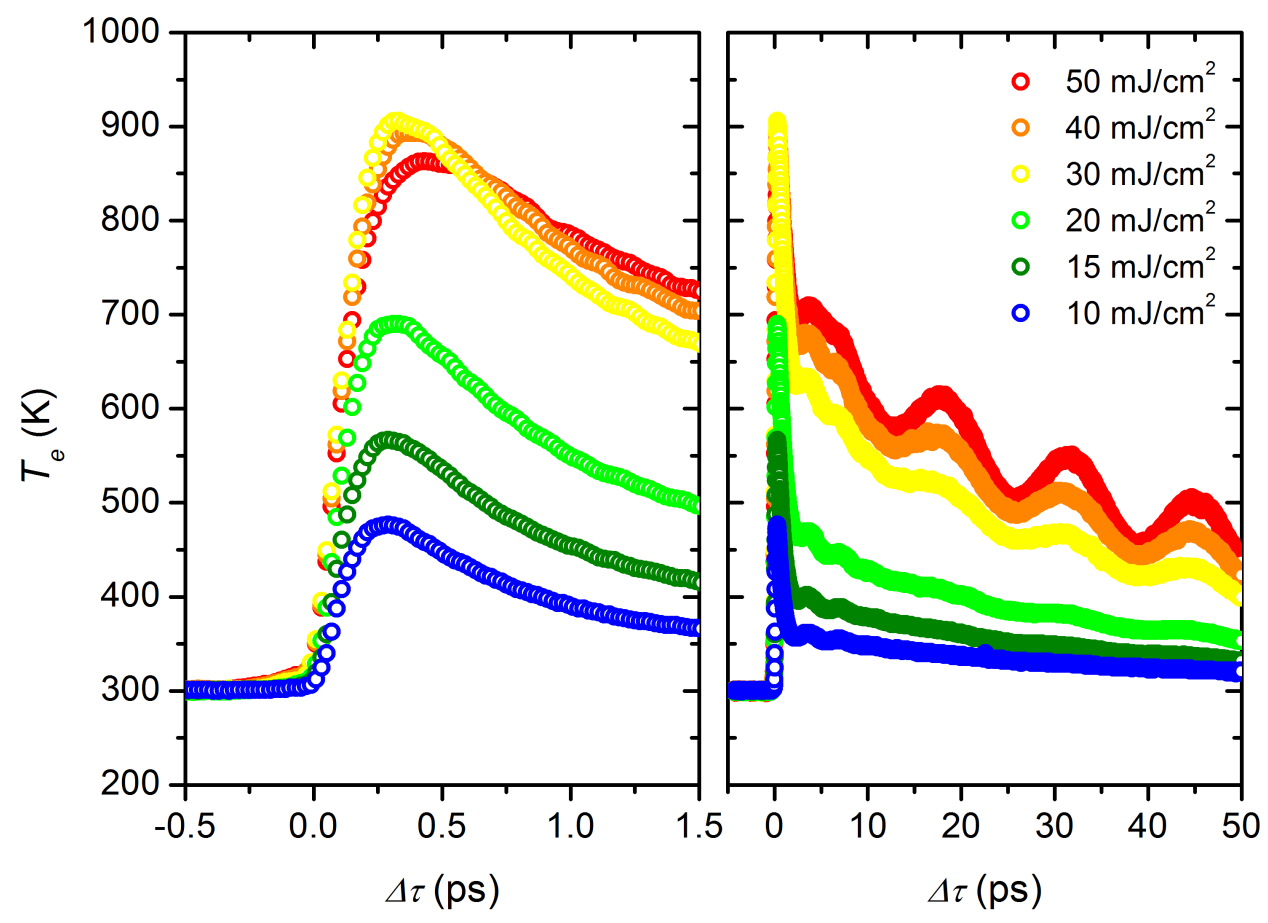

Figure B.9: Time-resolved temperature change in a $10 \mathrm{~nm}$ nickel film. Rescaled from the measured reflectivity data, by the procedure described in section 1.3 .3 .

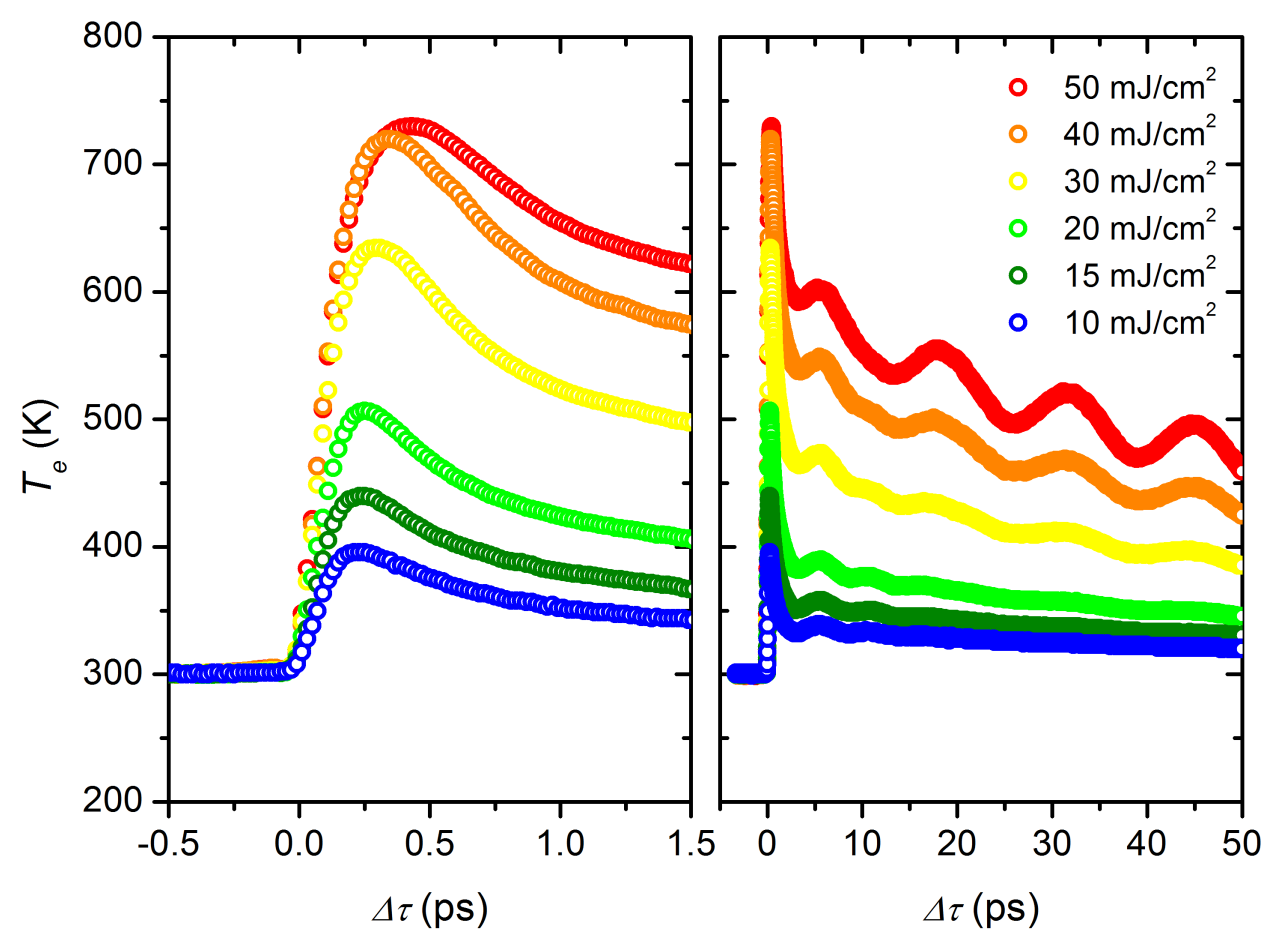

Figure B.10: Time-resolved temperature change in a $15 \mathrm{~nm}$ nickel film. Rescaled from the measured reflectivity data, by the procedure described in section 1.3.3. 
B TRMOKE and Time-Resolved $\Delta R$ Experimental Data

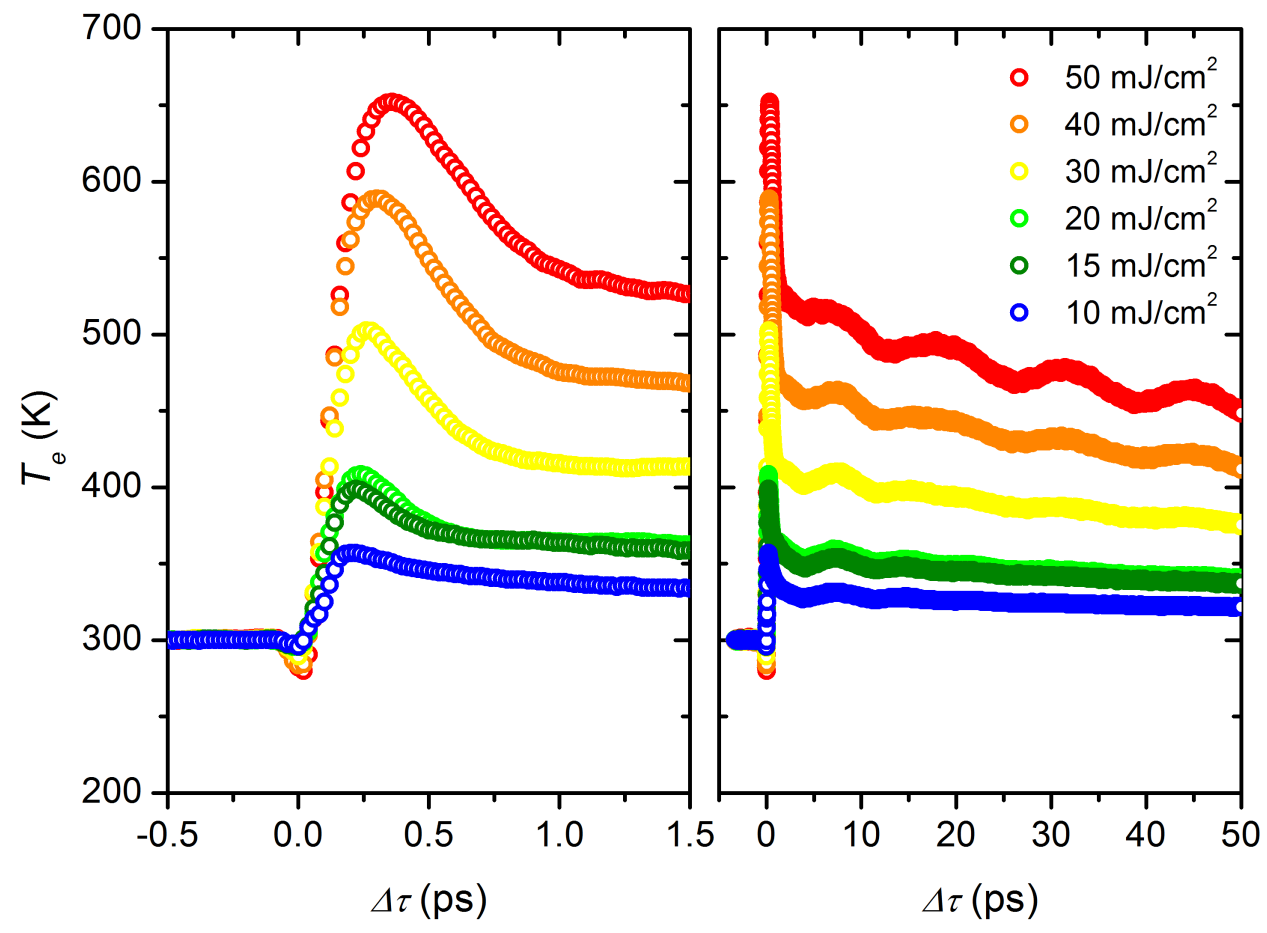

Figure B.11: Time-resolved temperature change in a $20 \mathrm{~nm}$ nickel film. Rescaled from the measured reflectivity data, by the procedure described in section 1.3 .3 .
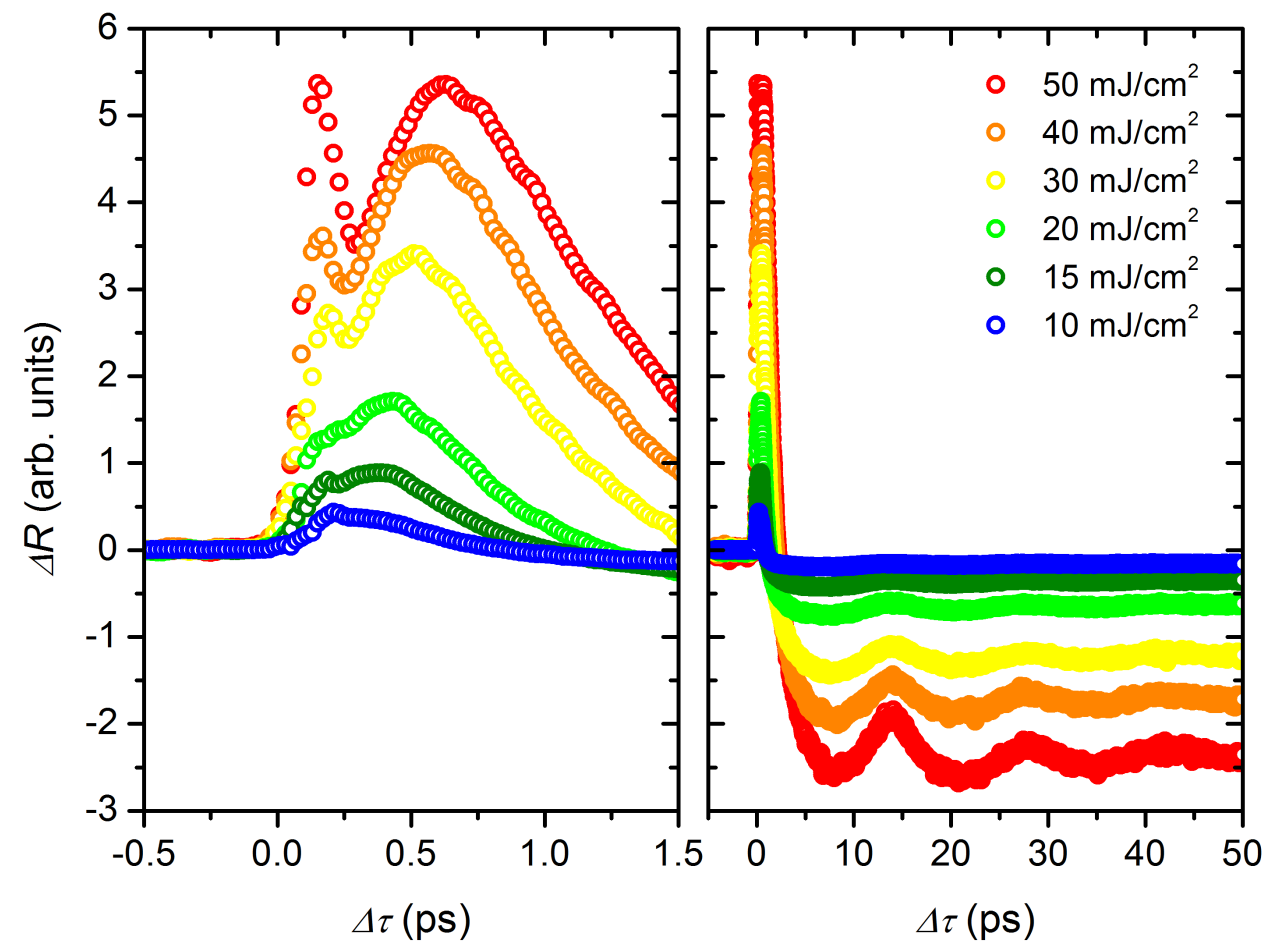

Figure B.12: Time-resolved reflectivity change in a $40 \mathrm{~nm}$ nickel film. Temperature calibration with the assumption $\Delta R(t) \sim T(t)$ is not possible. 



\section{Magneto-Seebeck Setup and Sample Description}

In the following, a detailed description of the examined samples is given. Figure C.1 shows the top view of a typical $1 \times 1 \mathrm{~cm}$ thermally oxidized silicon substrate with the tunnel junction distribution. In each row, the element sizes are the same, decreasing from top to bottom row. Every element is situated underneath an $\mathrm{Au}$ contact pad, which has two squared areas, to which wires can be contacted. The $\mathrm{Au}$ frame makes the bottom contact to all tunnel junctions, as shown in figure 2.10 in chapter 2.5.3. It has a large enough area to bond contact wires on top.

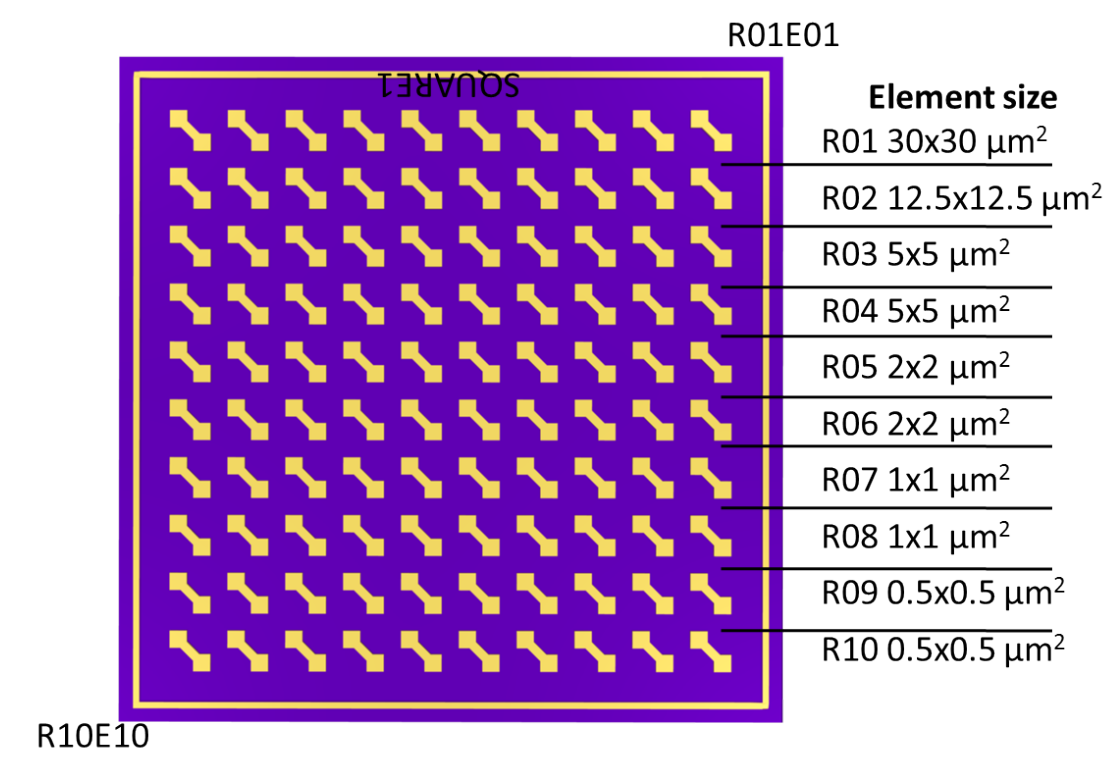

Figure C.1: Distribution of the tunnel junctions and their sizes on the sample.

Table C.1 shows the difference of the layer stacks of the examined samples. The tunnel junctions in all three samples have the same thicknesses. The main difference is given by the annealing parameters. In [68] it was found out that samples annealed at lower temperatures and low annealing times, as performed on sample vz101021b, show the highest TMR ratios, because then the diffusion of Ta into the junction area is the smallest. However, also higher annealing temperatures, up to $550^{\circ}$ often led to the same results, as long as the annealing time remained low, around $20 \mathrm{~min}$. High annealing times, around 60 min could also lead to Ta diffusion into the junction 
area, even at lower annealing temperatures. Therefore, the sample vz101007b lead to the best results in TMR measurements, showing the highest TMR ratio and distinguished switching behavior (between parallel and antiparallel alignment) and also the best results in the magneto-Seebeck measurements, presented above.

\begin{tabular}{|l|c|c|c|}
\hline Material/Layer/Procedure & mw100915b & vz101007b & vz101021b \\
\hline \hline $\mathrm{SiO}_{2}$ & Substrate & Substrate & Substrate \\
\hline \hline $\mathrm{Ta}$ & $5 \mathrm{~nm}$ & $6.2 \mathrm{~nm}$ & $6.2 \mathrm{~nm}$ \\
\hline \hline $\mathrm{CoFeB}$ & $2.5 \mathrm{~nm}$ & $2.5 \mathrm{~nm}$ & $2.5 \mathrm{~nm}$ \\
\hline $\mathrm{MgO}$ & $2.1 \mathrm{~nm}$ & $2.1 \mathrm{~nm}$ & $2.1 \mathrm{~nm}$ \\
\hline $\mathrm{CoFeB}$ & $5.4 \mathrm{~nm}$ & $5.4 \mathrm{~nm}$ & $5.4 \mathrm{~nm}$ \\
\hline \hline $\mathrm{Ta}$ & $5 \mathrm{~nm}$ & $5 \mathrm{~nm}$ & $5 \mathrm{~nm}$ \\
\hline $\mathrm{Ru}$ & $3 \mathrm{~nm}$ & $3 \mathrm{~nm}$ & $3 \mathrm{~nm}$ \\
\hline \hline Annealing Temperature & $450^{\circ} \mathrm{C}$ & $550^{\circ} \mathrm{C}$ & $450^{\circ} \mathrm{C}$ \\
\hline Annealing Field & $300 \mathrm{mT}$ & $300 \mathrm{mT}$ & $300 \mathrm{mT}$ \\
\hline Annealing Time & $60 \mathrm{~min}$ & $20 \mathrm{~min}$ & $20 \mathrm{~min}$ \\
\hline
\end{tabular}

Table C.1: Data of the examined samples and the annealing process. Samples named mw... were produced by Marvin Walter, samples named vz... were produced by Vladyslav Zbarsky. 


\section{Bibliography}

[1] S. I. Anisimov, B. L. Kapeliovich, and T. L. Perel'man. Electron emission from metal surfaces exposed to ultrashort laser pulses. Sov. Phys. JETP, 39 (2):375-377, 1974.

[2] Neil W. Ashcroft and N. David Mermin. Solid State Physics. Brooks/Cole, [South Melbourne], 1976. ISBN 0030839939.

[3] U. Atxitia and O. Chubykalo-Fesenko. Ultrafast magnetization dynamics rates within the Landau-Lifshitz-Bloch model. Physical Review B, 84(144414), 2011. ISSN 1098-0121. doi: 10.1103/PhysRevB.84.144414.

[4] U. Atxitia and O. Chubykalo-Fesenko. Unpublished, private communication, 2011.

[5] U. Atxitia, O. Chubykalo-Fesenko, N. Kazantseva, D. Hinzke, U. Nowak, and R. W. Chantrell. Micromagnetic modeling of laser-induced magnetization dynamics using the Landau-Lifshitz-Bloch equation. Applied Physics Letters, 91 (23):232507, 2007. ISSN 00036951. doi: 10.1063/1.2822807.

[6] U. Atxitia, O. Chubykalo-Fesenko, J. Walowski, A. Mann, and M. Münzenberg. Evidence for thermal mechanisms in laser-induced femtosecond spin dynamics. Physical Review B, 81(174401), 2010. ISSN 1098-0121. doi: 10.1103/PhysRevB. 81.174401.

[7] M. N. Baibich, J. M. Broto, A. Fert, F. Nguyen Van Dau, F. Petroff, P. Eitenne, G. Creuzet, A. Friedrich, and J. Chazelas. Giant Magnetoresistance of (001)Fe/(001)Cr Magnetic Superlattices. Physical Review Letters, 61 (21):2472-2475, 1988. doi: 10.1103/PhysRevLett.61.2472.

[8] M. Battiato, K. Carva, and P. Oppeneer. Superdiffusive Spin Transport as a Mechanism of Ultrafast Demagnetization. Physical Review Letters, 105 (027203), 2010. ISSN 0031-9007. doi: 10.1103/PhysRevLett.105.027203.

[9] M. Battiato, P. Maldonado, and P. M. Oppeneer. Unpublished, private communication, 2011.

[10] E. Beaurepaire, J.-C Merle, A. Daunois, and J.-Y Bigot. Ultrafast Spin Dynamics in Ferromagnetic Nickel. Physical Review Letters, 76(22):4250-4253, 1996. ISSN 0031-9007. doi: 10.1103/PhysRevLett.76.4250. 


\section{Bibliography}

[11] G. Binasch, P. Grünberg, F. Saurenbach, and W. Zinn. Enhanced magnetoresistance in layered magnetic structures with antiferromagnetic interlayer exchange. Physical Review B, 39(7):4828-4830, 1989. doi: 10.1103/PhysRevB.39.4828.

[12] W. Butler, X.-G Zhang, T. Schulthess, and J. MacLaren. Spin-dependent tunneling conductance of $\mathrm{Fe}-\mathrm{MgO}$ - Fe sandwiches. Physical Review B, 63 (054416), 2001. ISSN 1098-0121. doi: 10.1103/PhysRevB.63.054416.

[13] H. Callen. The Application of Onsager's Reciprocal Relations to Thermoelectric, Thermomagnetic, and Galvanomagnetic Effects. Physical Review, 73(11): 1349-1358, 1948. ISSN 0031-899X. doi: 10.1103/PhysRev.73.1349.

[14] Herbert B. Callen. Thermodynamics: an introduction to the physical theories of equilibrium thermostatics and irreversible thermodynamics. John Wiley \& Sons Inc., New York [u.a.], 1960. ISBN 0471130354.

[15] K. Carva, M. Battiato, and P. Oppeneer. Ab-Initio Investigation of the ElliottYafet Electron-Phonon Mechanism in Laser-Induced Ultrafast Demagnetization. Physical Review Letters, 107(207201), 2011. ISSN 0031-9007. doi: 10.1103/PhysRevLett.107.207201.

[16] O. Chubykalo-Fesenko, U. Nowak, R. Chantrell, and D. Garanin. Dynamic approach for micromagnetics close to the curie temperature. Physical Review B, 74(094436), 2006. ISSN 1098-0121. doi: 10.1103/PhysRevB.74.094436.

[17] Michael Czerner, Michael Bachmann, and Christian Heiliger. Spin caloritronics in magnetic tunnel junctions: Ab initio studies. Physical Review B, 83(132405), 2011. ISSN 1098-0121. doi: 10.1103/PhysRevB.83.132405.

[18] F. Dalla Longa, J. Kohlhepp, W. de Jonge, and B. Koopmans. Influence of photon angular momentum on ultrafast demagnetization in nickel. Physical Review B, 75(224431), 2007. ISSN 1098-0121. doi: 10.1103/PhysRevB.75. 224431.

[19] Francesco Dalla Longa. Laser-induced magnetization dynamics: an ultrafast journey among spins and light pulses. PhD thesis, Technische Universiteit Eindhoven, Eindhoven, 2008.

[20] M. Djordjevic, M. Lüttich, P. Moschkau, P. Guderian, T. Kampfrath, R. G. Ulbrich, M. Münzenberg, W. Felsch, and J. S. Moodera. Comprehensive view on ultrafast dynamics of ferromagnetic films. physica status solidi (c), 3(5): 1347-1358, 2006. ISSN 1610-1634. doi: 10.1002/pssc.200563123.

[21] Marija Djordjevic-Kaufmann. Magnetization dynamics in all-optical pumpprobe experiments: spin-wave modes and spin-current damping. PhD thesis, Georg-August-Universität, Göttingen, 2006. 
[22] Gerrit Eilers. Grundlegende Untersuchungen zu Tunnelmagnetowiderstandselementen: Schichtrauigkeit, Tunnelbarriere und magnetische Simulation. Diplomarbeit, Georg-August-Universität, Göttingen, 2004.

[23] Gerrit Eilers. Korrelationen zwischen struktureller Ordnung und elektrischen Transporteigenschaften in $\mathrm{CoFe} B|\mathrm{MgO}| \mathrm{CoFeB}$ Tunnelmagnetowiderstandselementen. PhD thesis, Georg-August-Universität, Göttingen, 2010.

[24] R. J. Elliott. Theory of the Effect of Spin-Orbit Coupling on Magnetic Resonance in Some Semiconductors. Physical Review, 96(2):266-279, 1954. ISSN 0031-899X. doi: 10.1103/PhysRev.96.266.

[25] D. A. Garanin. Generalized equation of motion for a ferromagnet. Physica A: Statistical and Theoretical Physics, 172(3):470-491, 1991. ISSN 03784371. doi: 10.1016/0378-4371(91)90395-S.

[26] D. A. Garanin. Fokker-Planck and Landau-Lifshitz-Bloch equations for classical ferromagnets. Physical Review B, 55(5):3050, 1997. ISSN 1098-0121.

[27] L. Gravier, A. Fábián, A. Rudolf, A. Cachin, K. Hjort, and J-Ph Ansermet. Thermopower measurement of single isolated metallic nanostructures. Measurement Science and Technology, 15(2):420, 2004.

[28] L. Gravier, S. Serrano-Guisan, F. Reuse, and J. P. Ansermet. Thermodynamic description of heat and spin transport in magnetic nanostructures. Physical Review B, 73(2), 2006. ISSN 1098-0121. doi: 10.1103/PhysRevB.73.024419.

[29] Eugene Hecht. Optik. Oldenbourg, München and Wien, 4., überarb. edition, 2005. ISBN 9783486273595.

[30] Christian Heiliger, Michael Czerner, Bogdan Yu Yavorsky, Ingrid Mertig, and Mark D. Stiles. Implementation of a nonequilibrium Green's function method to calculate spin-transfer torque. Journal of Applied Physics, 103(7):07A709, 2008. ISSN 00218979. doi: 10.1063/1.2835071.

[31] J. Hirsch. Spin Hall Effect. Physical Review Letters, 83(9):1834-1837, 1999. ISSN 0031-9007. doi: 10.1103/PhysRevLett.83.1834.

[32] Jisang Hong and D. Mills. Spin dependence of the inelastic electron mean free path in Fe and Ni: Explicit calculations and implications. Physical Review B, 62(9):5589-5600, 2000. ISSN 1098-0121. doi: 10.1103/PhysRevB.62.5589.

[33] C. M. Jaworski, J. Yang, S. Mack, D. D. Awschalom, J. P. Heremans, and R. C. Myers. Observation of the spin-Seebeck effect in a ferromagnetic semiconductor. Nature Materials, 9(11):898-903, 2010. ISSN 1476-1122. doi: 10.1038/NMAT2860. 


\section{Bibliography}

[34] X. Jia, K. Xia, and G. Bauer. Thermal Spin Transfer in Fe $|\mathrm{MgO}| \mathrm{Fe}$ Tunnel Junctions. Physical Review Letters, 107(176603), 2011. ISSN 0031-9007. doi: 10.1103/PhysRevLett.107.176603.

[35] M. Johnson and R. H. Silsbee. Interfacial charge-spin coupling: Injection and detection of spin magnetization in metals. Physical Review Letters, 55(17): 1790-1793, 1985. ISSN 0031-9007. doi: 10.1103/PhysRevLett.55.1790.

[36] M. Johnson and R. H. Silsbee. Thermodynamic analysis of interfacial transport and of the thermomagnetoelectric system. Physical Review B, 35(10):49594972, 1987. ISSN 1098-0121.

[37] N. Kazantseva, D. Hinzke, U. Nowak, R. Chantrell, U. Atxitia, and O. Chubykalo-Fesenko. Towards multiscale modeling of magnetic materials: Simulations of FePt. Physical Review B, 77(184428), 2008. ISSN 1098-0121. doi: 10.1103/PhysRevB.77.184428.

[38] B. Koopmans, G. Malinowski, F. Dalla Longa, D. Steiauf, M. Fähnle, T. Roth, M. Cinchetti, and M. Aeschlimann. Explaining the paradoxical diversity of ultrafast laser-induced demagnetization. Nature Materials, 9(3):259-265, 2010. ISSN 1476-1122. doi: 10.1038/nmat2593.

[39] Bert Koopmans. Laser-Induced Magnetization Dynamics. In Burkard Hillebrands and Kamel Ounadjela, editors, Spin dynamics in confined magnetic structures, volume 87, pages 256-323. Springer, Berlin, 2003. ISBN 9783540440840 .

[40] K. C. Kuiper, G. Malinowski, F. Dalla Longa, and B. Koopmans. Nonlocal ultrafast magnetization dynamics in the high fluence limit. Journal of Applied Physics, 109(7):07D316, 2011. ISSN 00218979. doi: 10.1063/1.3540681.

[41] B. Lenk, H. Ulrichs, F. Garbs, and M. Münzenberg. The building blocks of magnonics. Physics Reports, 507(4-5):107-136, 2011. ISSN 03701573. doi: 10.1016/j.physrep.2011.06.003.

[42] N. Liebing, S. Serrano-Guisan, K. Rott, G. Reiss, J. Langer, B. Ocker, and H. Schumacher. Tunneling Magnetothermopower in Magnetic Tunnel Junction Nanopillars. Physical Review Letters, 107(177201), 2011. ISSN 0031-9007. doi: 10.1103/PhysRevLett.107.177201.

[43] W. Lin, M. Hehn, L. Chaput, B. Negulescu, S. Andrieu, F. Montaigne, and S. Mangin. Giant thermoelectric effect in $\mathrm{Al}_{2} \mathrm{O}_{3}$ magnetic tunnel junctions. arXiv:1109.3421v2, 2011.

[44] Martin Lüttich. Einfluss der Pulsdauer auf die Relaxation zum thermischen Gleichgewicht bei Entmagnetisierungsuntersuchungen auf der Femtosekundenzeitskala. Bachelorarbeit, Gerog-August-Universität, Göttingen, 2011. 
[45] Andreas Mann. Determining the Spin Polarization of Heusler Compounds via Femtosecond Magnetization Dynamics. Diplomarbeit, Georg-AugustUniversität, Göttingen, 2010.

[46] S. Mizukami, F. Wu, A. Sakuma, J. Walowski, D. Watanabe, T. Kubota, X. Zhang, H. Naganuma, M. Oogane, Y. Ando, and T. Miyazaki. Long-Lived Ultrafast Spin Precession in Manganese Alloys Films with a Large Perpendicular Magnetic Anisotropy. Physical Review Letters, 106(117201), 2011. ISSN 0031-9007. doi: 10.1103/PhysRevLett.106.117201.

[47] G. M. Müller, J. Walowski, M. Djordjevic, G. X. Miao, A. Gupta, A. V. Ramos, K. Gehrke, V. Moshnyaga, K. Samwer, J. Schmalhorst, A. Thomas, A. Hütten, G. Reiss, J. S. Moodera, and M. Münzenberg. Spin polarization in half-metals probed by femtosecond spin excitation. Nature Materials, 8(1):56-61, 2008. ISSN 1476-1122. doi: 10.1038/nmat2341.

[48] L. Onsager. Reciprocal Relations in Irreversible Processes. I. Physical Review, 37(4):405-426, 1931. ISSN 0031-899X. doi: 10.1103/PhysRev.37.405.

[49] E.D Palik and G. Ghosh. Handbook of optical constants of solids. Academic Press, 1998. ISBN 9780125444200.

[50] R. Pelster, I. Hüttl, and R. Pieper. Thermospannungen - viel genutzt und fast immer falsch erklärt. Physik und Didaktik in Schule und Hochschule, 1(4): 10-22, 2005.

[51] Thomas Rademacher. Strukturelle Untersuchung an massivem und nanokristallinem ternärem $\mathrm{Fe}_{3} \mathrm{Al}$. $\mathrm{PhD}$ thesis, Georg-August-Universität, Göttingen, 2011.

[52] I. Radu, K. Vahaplar, C. Stamm, T. Kachel, N. Pontius, H. A. Dürr, T. A. Ostler, J. Barker, R. F. L. Evans, R. W. Chantrell, A. Tsukamoto, A. Itoh, A. Kirilyuk, Th Rasing, and A. V. Kimel. Transient ferromagnetic-like state mediating ultrafast reversal of antiferromagnetically coupled spins. Nature, 472 (7342):205-208, 2011. ISSN 0028-0836. doi: 10.1038/nature09901.

[53] H.-S Rhie, H. Dürr, and W. Eberhardt. Femtosecond Electron and Spin Dynamics in Ni/W(110) Films. Physical Review Letters, 90(247201), 2003. ISSN 0031-9007. doi: 10.1103/PhysRevLett.90.247201.

[54] R. Schoenlein, W. Lin, J. Fujimoto, and G. Eesley. Femtosecond studies of nonequilibrium electronic processes in metals. Physical Review Letters, 58(16): 1680-1683, 1987. ISSN 0031-9007. doi: 10.1103/PhysRevLett.58.1680.

[55] Thomas Johann Seebeck. Magnetische Polarisation der Metalle und Erze durch Temperaturdifferenz. Abhandlungen der Königlich Preußischen Akademie der Wissenschaften zu Berlin, pages 265-375, 1822-1823. 


\section{Bibliography}

[56] U. Sivan and Y. Imry. Multichannel Landauer formula for thermoelectric transport with application to thermopower near the mobility edge. Physical Review B, 33(1):551-558, 1986. ISSN 1098-0121. doi: 10.1103/PhysRevB.33.551.

[57] C. Stamm, T. Kachel, N. Pontius, R. Mitzner, T. Quast, K. Holldack, S. Khan, C. Lupulescu, E. F. Aziz, M. Wietstruk, H. A. Dürr, and W. Eberhardt. Femtosecond modification of electron localization and transfer of angular momentum in nickel. Nature Materials, 6(10):740-743, 2007. ISSN 1476-1122. doi: 10.1038/nmat1985.

[58] D. Steiauf and M. Fähnle. Elliott-Yafet mechanism and the discussion of femtosecond magnetization dynamics. Physical Review B, 79(140401), 2009. ISSN 1098-0121. doi: 10.1103/PhysRevB.79.140401.

[59] Joachim Stöhr and Hans Christoph Siegmann. Magnetism: From fundamentals to nanoscale dynamics. Springer, Berlin and and Heidelberg, 2006. ISBN 9783540302827.

[60] K. Uchida, S. Takahashi, K. Harii, J. Ieda, W. Koshibae, K. Ando, S. Maekawa, and E. Saitoh. Observation of the spin Seebeck effect. Nature, 455(7214):778781, 2008. ISSN 0028-0836. doi: 10.1038/nature07321.

[61] K. Uchida, J. Xiao, H. Adachi, J. Ohe, S. Takahashi, J. Ieda, T. Ota, Y. Kajiwara, H. Umezawa, H. Kawai, G. E. W. Bauer, S. Maekawa, and E. Saitoh. Spin Seebeck insulator. Nature Materials, 9(11):894-897, 2010. ISSN 1476-1122. doi: 10.1038/nmat2856.

[62] H. Ulrichs, B. Lenk, and M. Münzenberg. Magnonic spin-wave modes in CoFeB antidot lattices. Applied Physics Letters, 97(9):092506, 2010. ISSN 00036951. doi: $10.1063 / 1.3483136$.

[63] Jakob Walowski. Non-Local/Local Gilbert Damping in Nickel and Permalloy Thin Films. Diplomarbeit, Georg-August-Universität, Göttingen, 2007.

[64] M. Walter, J. Walowski, V. Zbarsky, M. Münzenberg, M. Schäfers, D. Ebke, G. Reiss, A. Thomas, P. Peretzki, M. Seibt, J. S. Moodera, M. Czerner, M. Bachmann, and C. Heiliger. Seebeck effect in magnetic tunnel junctions. $\mathrm{Na}-$ ture Materials, 10(10):742-746, 2011. ISSN 1476-1122. doi: 10.1038/nmat3076.

[65] M. Wietstruk, A. Melnikov, C. Stamm, T. Kachel, N. Pontius, M. Sultan, C. Gahl, M. Weinelt, H. Dürr, and U. Bovensiepen. Hot-Electron-Driven Enhancement of Spin-Lattice Coupling in Gd and Tb 4f Ferromagnets Observed by Femtosecond X-Ray Magnetic Circular Dichroism. Physical Review Letters, 106(127401), 2011. ISSN 0031-9007. doi: 10.1103/PhysRevLett.106.127401.

[66] Y. Yafet. g Factors and Spin-Lattice Relaxation of Conduction Electrons. In Solid State Physics, volume 14, pages 1-98. Elsevier, 1963. ISBN 9780126077148. doi: 10.1016/S0081-1947(08)60259-3. 
[67] S. Yuasa and D. D. Djayaprawira. Giant tunnel magnetoresistance in magnetic tunnel junctions with a crystalline $\mathrm{MgO}(001)$ barrier. Journal of Physics D: Applied Physics, 40(21):R337-R354, 2007. ISSN 0022-3727. doi: 10.1088/ 0022-3727/40/21/R01.

[68] Vladislav Zbarsky. Charakterisierung und Optimierung magnetischer Tunnelelemente. Diplomarbeit, Georg-August-Universität, Göttingen, 2011.

[69] Anissa Zeghuzi. Magneto-Seebeck Effekt: Simulationen und Experimente zum Temperaturverlauf. Bachelorarbeit, Gerog-August-Universität, Göttingen, 2011.

[70] V. Zhukov, E. Chulkov, and P. Echenique. Lifetimes and inelastic mean free path of low-energy excited electrons in Fe, Ni, Pt, and $\mathrm{Au}$ : Ab initio GW+T calculations. Physical Review B, 73(125105), 2006. ISSN 1098-0121. doi: 10. 1103/PhysRevB.73.125105. 



\section{Danksagung}

An dieser Stelle möchte ich mich ganz herzlich bei allen bedanken, die mich bei der Entstehung dieser Arbeit begleitet und unterstützt haben.

Ganz besonderer Dank geht an Prof. Markus Münzenberg für die Betreuung während der Zeit meiner Promotion. Die Mitarbeit in seiner Arbeitsgruppe war sehr lehrreich und hat viel Spaß gemacht. Eine offene Tür, die permanente Diskussionsbereitschaft und motivierende Gespräche und nahezu grenzenlose Geduld sorgten für ein angenehmes Arbeitsklima und ein stetiges Fortschreiten der Arbeit. Ferner möchte ich mich bei ihm für die Förderung von Teilnahmen an diversen Summerschools und den aktiven Austausch mit anderen Arbeitsgruppen bedanken, die den Blick auf die eigene Arbeit geschärft haben.

Bei Prof. Konrad Samwer, möchte ich mich für die Übernahme des Korreferats und die fördernde Mitwirkung an unserer Arbeit bedanken.

Ein ganz großer Dank geht an die Mitarbeiter ("Mitspinler") der AG Münzenberg, Gerrit Eilers, Benjamin Lenk, Marvin Walter, Andreas Mann und Vladyslav Zbarsky, die immer für eine konstruktive und angenehme Arbeitsatmosphäre gesorgt haben. Im Besonderen gilt der Dank Gerrit für die kompetente technische Beratung bei den Experimenten, Benny für die Instandhaltung und ständige Verbesserung des Femtosekundenlabors, Marv für die beste Zeit im Labor bei der Arbeit an MagnetoSeebeck Experimenten, Andreas für die Zusammenarbeit bei den Magnetisierungsdynamikmessungen und Vlad für die Herstellung von TMR Proben. Auch den übrigen, namentlich nicht erwähnten Mitarbeitern gilt großer Dank für die tolle Zeit und Zusammenarbeit.

Weiterer Dank geht an unsere Kooperationspartner in Spanien, Unai Atxitia und Schweden, Marco Battiato, für die Simulationen der Magnetisierungsdynamik und die Diskussionen der Ergebnisse.

Ich danke den technischen Mitarbeitern des I. und des IV. Physikalischen Instituts für die schnellen Abhilfen bei Problemen mit der Technik, Herrn Eichenberg und Herrn Gatzemeier aus der Feinmechanik Werkstatt für das Herstellen von benötigten Bauteilen und Carsten Mahn für die Versorgung mit elektronischen Bauteilen.

Vielen Dank an Benjamin Lenk und Marvin Walter für das Korrekturlesen der Manuskripte und die vielen konstruktiven Verbesserungsvorschläge.

Bei Prof. Yasuo Ando und Prof. Terunobu Miyazaki und den Mitarbeitern ihrer Arbeitsgruppen an der Tohoku University möchte mich für den angenehmen Aufenthalt in Sendai bedanken. Speziell Shigemi Mizukami für die gute Zusammenarbeit im Labor und Mikihiko Oogane für die Wohnungssuche und die Unterstützung mit der Administration vor Ort gilt mein besonderer Dank.

Dem DAAD gilt der Dank für die finanzielle Unterstützung und Ermöglichung 
der Zusammenarbeit mit den Arbeitsgruppen der Tohoku University. Dazu noch ein besonderer Dank an Prof. Wolfgang Felsch für die Unterstützung bei der Bewerbung um das DAAD Stipendium.

Schließlich möchte ich mich ganz besonders bei meiner Frau und meinem Sohn für die emotionale und moralische Unterstützung und die riesige Freude speziell in den vergangenen sechs Monaten und bei meinen Eltern und meiner Schwester für die Unterstützung in allen Lebenslagen bedanken. 


\section{Lebenslauf}

\section{Persönliche Daten}

Name:

Geburtsdatum:

Gebrutsort:

Familienstand:

Staatsangehörigkeit:
Jakob Walowski

4. März 1980

Ketrzyn (Polen)

verheiratet

deutsch

\section{Schulbildung}

1987-1989

1990-1991

1991-1993

1993-2000

2000

\section{Studium}

2001-2007

2007

seit 2007:

seit 2008:

2009
Grundschule (Polen)

Grundschule Göttingen

Orientierungsstufe Göttingen

Otto-Hahn-Gymnasium Göttingen

Abitur

Physikstudium an der Universität Göttingen

Diplomarbeit bei Prof. Dr. Markus Münzenberg Non-Local/Local Gilbert Damping in Nickel and Permalloy Thin Films

Wissenschaftlicher Mitarbeiter am IV. Physikalischen Institut der Georg-August-Universität Göttingen

Wissenschaftlicher Mitarbeiter am I. Physikalischen Institut der Georg-August-Universität Göttingen

DAAD Kurzstipendium für Doktoranden:

6 monatiger Forschungsaufenthalt an der

Tohoku University Sendai (Japan) in zusammenarbeit mit

Prof. Y. Ando, Prof. T. Miyazaki und Prof. S. Mizukami 


